Thorben Mämecke

\title{
DAS \\ QUANTIFIZIERTE \\ SELBST
}

Zur Genealogie des Self-Trackings 
Thorben Mämecke

Das quantifizierte Selbst

Digitale Gesellschaft | Band 34 
Dr. Thorben Mämecke ist wissenschaftlicher Geschäftsführer des Forschungsschwerpunktes digitale_kultur an der FernUniversität Hagen. Er forscht und lehrt mit besonderem Fokus auf Subjektivierung und Gouvernementalität im Kontext von progressiven Technologiediskursen, Dataveillance und Verhaltenskontrolle. www.dasquantifizierteselbst.de 
Thorben Mämecke

Das quantifizierte Selbst

Zur Genealogie des Self-Trackings

[transcript] 
Diese Publikation wurde im Rahmen des Fördervorhabens 16TOA002 mit Mitteln des Bundesministerium für Bildung und Forschung im Open Access bereitgestellt.

Diese Monografie wurde 2018 an der Fakultät für Kulturwissenschaften der Universität Paderborn als Dissertation angenommen.

\section{Bibliografische Information der Deutschen Nationalbibliothek}

Die Deutsche Nationalbibliothek verzeichnet diese Publikation in der Deutschen Nationalbibliografie; detaillierte bibliografische Daten sind im Internet über http://dnb.d-nb.de abrufbar.

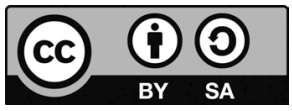

Dieses Werk ist lizenziert unter der Creative Commons Attribution-ShareAlike 4.0 Lizenz (BY-SA). Diese Lizenz erlaubt unter Voraussetzung der Namensnennung des Urhebers die Bearbeitung, Vervielfältigung und Verbreitung des Materials in jedem Format oder Medium für beliebige Zwecke, auch kommerziell, sofern der neu entstandene Text unter derselben Lizenz wie das Original verbreitet wird. (Lizenz-Text:

https://creativecommons.org/licenses/by-sa/4.0/deed.de)

Die Bedingungen der Creative-Commons-Lizenz gelten nur für Originalmaterial. Die Wiederverwendung von Material aus anderen Quellen (gekennzeichnet mit Quellenangabe) wie z.B. Schaubilder, Abbildungen, Fotos und Textauszüge erfordert ggf. weitere Nutzungsgenehmigungen durch den jeweiligen Rechteinhaber.

\section{Erschienen 2021 im transcript Verlag, Bielefeld (ㄷ) Thorben Mämecke}

Umschlaggestaltung: Maria Arndt, Bielefeld Umschlagcredit: $D Q S$ « von Thorben Mämecke Druck: Majuskel Medienproduktion GmbH, Wetzlar

Print-ISBN 978-3-8376-5603-9

PDF-ISBN 978-3-8394-5603-3

EPUB-ISBN 978-3-7328-5603-9

https://doi.org/10.14361/9783839456033

Gedruckt auf alterungsbeständigem Papier mit chlorfrei gebleichtem Zellstoff. Besuchen Sie uns im Internet: https://www.transcript-verlag.de Unsere aktuelle Vorschau finden Sie unter www.transcript-verlag.de/vorschau-download 


\section{Inhalt}

\section{Einleitung}

$1 \quad$ Was ist Self-Tracking? ............................................. 11

2 Das Forschungsprogramm ........................................ 15

\section{Methodisches Vorgehen}

\section{Die Beschreibung progressiver Selbstverdatung}

Self-Tracking im Schnittfeld von Diskursanalyse und Gouvernementalitätsstudien .... 25

1.1 Die Unordnung der Diskursforschung................................. 25

1.2 Zum Verhältnis von Gouvernementalitätsstudien und Diskursforschung ........... 26

1.3 Diskursanalyse als induktives Verfahren................................. 31

1.4 Aufsteigende Analyse und aufsteigende Methodenbildung ................... 32

2 Herausfinden, ob die Maschine läuft und was sie produziert

Das Instrumentarium der Feinanalyse ............................... 37

2.1 Gegenstände und Formationsregeln - Self-Tracking als Diskursgegenstand......... 38

2.2 Datentypen und numerische Äußerungsmodalitäten ...................... 40

2.3 Korpusbildung - Die selbstregistrative Praxis des Self-Trackings................ 41

2.4 Herkunft und Ursprung - Diskursanalyse, Genealogie und Narrativität ............. 44

2.5 Populäre Ursprungsnarrative des Self-Trackings ...........................47

\section{Die Analyse des Self-Tracking-Diskurses}

\section{Von Quetelet bis Quantified Self}

Statistische Regulation im soziotechnischen Wandel...................... 55

1.1 Verdatung und Regulation - Von der Anthropometrie zur Sozialstatistik ........... 55

1.2 Verdatung und Selbstregulation - Reflexive Sozialstatistik und Verbreitungsmedien .. 60

1.3 Selbstverdatung und Selbstregulation - Statistik und interaktive Internetmedien .... 62 


\section{Self-Tracking im Schnittfeld progressiver Technologiekulturen}

und Kreativitätsdispositive ....................................... 65

2.1 Interdiskursbeziehungen: Technologieinnovation und Kreativwirtschaft ........... 70

2.2 Die diskursive Eigendynamik des Prekarisierungsbegriffs . ................... 84

2.3 Self-Tracking und Kreativwirtschaft .................................. 89

2.4 Datataining, Data Selfies, Data Artists .................................. 91

2.5 Datenmacht ......................................................... 104

2.5.1 Data is the new oil: Interdiskursbeziehungen zwischen Self-Tracking und Big Data ............................. 104

2.5.2 Data is not the new oil: Naturalisierung und Politisierung von Daten und ihrer Erzeugung ................................... 110

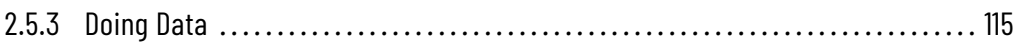

2.5.4 Self-Tracking und Privacy...................................... 119

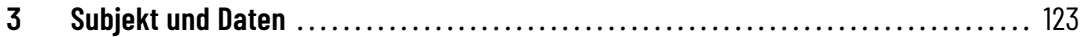

3.1 Das Selbst des Körpers und des Gefühls ............................. 124

3.2 Das Selbst der zeitlichen Entwicklung ................................... 128

3.3 Das Selbst der Routinen ........................................... 134

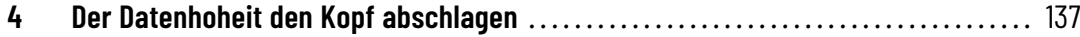

4.1 Optimierung und Empowerment bei Ouantified Self ......................... 137

4.2 Die Mechanik der Macht und die soziale Orthopädie des Taylorismus ............ 146

4.3 Über den »bebenden Sockel der Kräfteverhältnisse« Zur Ambivalenz des Begriffs der Arbeitskraft ............................... 152

4.4 »n praise of a paradox « - Der Freiheitszwang des Empowerment ................ 157

4.5 Selbstoptimierung oder »die Zeit anders leben « ........................... 164

4.6 Disziplin und Selbstdisziplin ......................................... 169

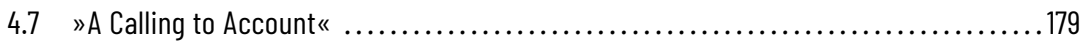

5 Quantrepreneure und der Individualismus der Prototypen ................... 183

5.1 Quantified Self und die Verbreitung von Self-Tracking-Technologien............... 183

5.2 A Community of like-minded others Individuelles Tracking und überindividuelle Vergleiche ..................... 188

5.3 Selbstwertsteigerung und Ressourcenplanung im Ich-Unternehmen ............... 192

5.4 Work/Workout-Balance - Resilienzstrategien im Home Office ................... 195

6 Selbstvermessung und Betriebliches Gesundheitsmanagement............... 201

6.1 Interdiskursbeziehungen: Burnout und Self-Tracking in Unternehmen .............. 203

6.2 Interdiskursbeziehungen: Quantified Self und Self-Tracking in Unternehmen ........ 209 
6.3 Belastung und Anpassung - Partizipative Approximation nachhaltiger Leistungsfähigkeit..................................... 217

6.4 Self-Tracking als Teil einer kompetetiven Selbstsorgekultur ....................221

6.5 Interdiskursbeziehungen: Self-Tracking und partizipatives Risikomanagement im Gesundheitswesen ..................... 225

\section{Schluss}

1 Progressive Selbstverdatung oder die Pflicht zum Wissen ................. 235

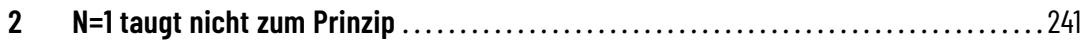

Literaturverzeichnis............................................. 249

Abkürzungsverzeichnis.............................................. 267 

I. Einleitung 



\section{Was ist Self-Tracking?}

Was sind Self-Tracking-Technologien und worin begründet sich ihre rasante Karriere, die sie in nur wenigen Jahren aus den Makerspaces in die App-Stores und das Inventar der Elektrofachgeschäfte befördert hat? Um diese Fragen beantworten zu können und dabei die Ebene der reinen Deskription zu verlassen, erscheint es zunächst vielversprechend, sich anzusehen, was diese Tracking-Technologien in ihrer Unterschiedlichkeit verbindet, worauf hin sie sich ausrichten und zur Erfüllung welcher Vorstellungen sie entwickelt und hergestellt werden. Oder genauer, was diese Technologien selbst herstellen. Die voraussetzungsvolle Antwort, die zugleich eine weiterführende Frage ist, gibt den Titel dieser Forschung vor: Das quantifizierte Selbst.

Dieses Selbst ist keine essentielle Kategorie. Kein Element eines im Innern von Menschen liegenden Wesens. Es ist vielmehr selbst ein heuristisches Konzept und beschreibt keine vorfindbare Entität.

Die Skizzierung des quantifizierten Selbstes erfolgt hier auch nicht auf der Ebene von Individuen und der Beobachtung ihrer Praktiken, sondern auf der Ebene ihres diskursiven Austausches. D.h. das quantifizierte Selbst wird im Folgenden subjekttheoretisch apostrophiert und als das vorläufige Ergebnis und das Ziel der Bemühungen, Zwänge, Deutungskämpfe und der Probleme betrachtet, die durch die Herstellung eines bestimmten Selbstbezugs gelöst werden sollen. Eine solche Analyse betrachtet Self-Tracking und quantifiziertes Selbst nicht als kausalen Zusammenhang, sondern als voneinander abhängige Elemente des gleichen Diskurses, wobei sie gerade die Netzwerkartigkeit und Verweisungsvielfalt dieser Elemente betont und sich der Suche nach genuinen Ursprüngen oder individuell identifizierbaren Produzent"innen verwehrt. Aus dieser Perspektive erscheint der Diskurs somit als »Produktionsort« dieses Selbstverhältnisses und der dafür angewendeten Technologien.

Schon seit einigen Jahren hat die Ausrufung von Selbsten durch poststrukturalistische Analysen und Theorien regelrecht Konjunktur. Das quantifizierte Selbst ist dieser Konjunktur sicher zuzurechnen, es ist aber kein antagonistisches Konzept, das an Stelle anderer Konzeptionen für sich beansprucht, die zentrale Erklärungsfolie für zeitgenössische gesellschaftliche Entwicklungsprozesse abbilden zu kön- 
nen. Es ist allerdings auch nicht auf einen singulären Technologieentwicklungsdiskurs reduzierbar; denn die Analyse die es konturiert beschränkt sich nicht im Sinne einer Organisationsforschung auf die formalen Strukturen der Communities die es umgeben. Das quantifizierte Selbst stellt auch nicht lediglich ein weiteres Exempel für die verschiedenen Varianten dar, in denen sich Selbstverständnisse als Ergebnis unternehmerischer Selbstführungs-, Kreativitäts- oder Gesundheitsimperative herausbilden. Vielmehr ist es eine Mischung aus Selbst- und Fremdbeschreibungen, die zeitgleich neben anderen Subjektivationszielen auf Individuen einwirken kann und auch nur in Überschneidung mit ihnen existiert.

So setzt es etwa die gesellschaftlichen Leitbilder der unternehmerischen Selbstrationalisierung ebenso voraus, wie die Imperative der kreativen Selbstverwirklichung oder die immer selbstverständlicher werdende Erwartung, das eigene Leben nach Maßgabe der körperlichen und geistigen Gesunderhaltung zu organisieren.

Die Skizzierung der Modi, in denen sich diese mitunter verschiedenen und teilweise gegenläufigen Erwartungen zu einer konsistenten Zielfolie für Selbstentwürfe verbinden, ist daher einer der Ansatzpunkte dieser Analyse.

Anders als die meisten gouvernementalitätstheoretisch ausgerichteten Analysen, die vornehmlich Agenturen und Institutionen in den Blick nehmen, die zu einer bestimmten Selbstwahrnehmung drängen, steht im Zentrum dieser Analyse, das Drängen des Diskurses selbst, in dem politische oder medizinische Autoritäten, Life-Coaches oder Ökonom*innen eine Rolle spielen, der sich aber nicht auf ihre Empfehlungen und Ermahnungen beschränkt.

Die Analyse des Self-Tracking-Diskurses trägt damit dem Umstand Rechnung, dass es lange offenkundig keine gouvernementale Agentur gab, die forderte: »Vermiss dich selbst!«Vielmehr beginnt die Analyse in entgegengesetzter Perspektive und rekonstruiert Teile des umfangreichen dezentralen Austauschprozesses, der nach und nach zur Entwicklung und Anwendung von Selbstvermessungstechnologien geführt hat, ehe das Self-Tracking schließlich auch zum Ziel von Vereinnahmungsstrategien z.B. gesundheitswirtschaftlicher Akteure wurde.

Eine Besonderheit dieses Diskurses liegt dabei darin, dass die Suche nach der Definition des quantifizierten Selbst eine Forschungsfrage ist, die auch die numerischen Selbsterforschungen des untersuchten Diskurses anleitet. Als QuantifiedSelf-Community beschreibt sich ein Zusammenschluss von Self-Tracker*innen, der sich nicht nur der Entwicklung, sondern auch an der Verbreitung von SelfTracking-Technologien verschrieben hat und der eine entscheidende Rolle in der formalen Organisation des Diskurses einnimmt. Schon an den Titeln ihrer Konferenzvorträge, Blog-Einträge oder Feuilleton-Artikel lässt sich deutlich ablesen, dass sie Selbstquantifizierungstechnologien als Emanzipationsstrategie und Möglichkeit zur Erlangung von Selbstbestimmtheit ansehen. Durch die Selbstbeschreibung als progressive Bewegung ergibt sich aus der induktiven Analyse, der 
im Diskurs des Selbstvermessens artikulierten Motive, so auch ein unmittelbarer Zusammenhang mit machttheoretischen Fragen.

Während das Versprechen der Quantified-Self-Community allerdings darin besteht, durch mehr Wissen über sich selbst auch mehr Macht zu erlangen, lässt sich durch eine an Foucault anschließende Analyse zeigen, auf welche Weise Macht vielmehr das Wissen formt, das durch Self-Tracking generiert wird.

Bei der Untersuchung der Frage wie die Begriffe Emanzipation und Selbstbestimmung im Self-Tracking-Diskurs mit Bedeutung ausgestattet werden und welche Wege für ihre Verwirklichung beschritten werden, tritt die Analyse in Distanz zu den im Diskurs vorherrschenden Motiven und rekontextualisiert dominante Narrative (wie z.B. den Fortschrittsoptimismus der Quantified-Self-Mitglieder, der Emanzipation und Selbstbestimmung mit Individualität gleichsetzt) als diskursive Subjektivierungsprozesse, die im Zeichen der Individualisierung neue Formen der Selbstüberwachung und -kontrolle forcieren.

Diese Verschiebung bewegt sich somit vor allem im Spannungsfeld zwischen Aspekten der Selbstbestimmung und der Selbstregierung. D.h. das quantifizierte Selbst fordert vor allem in den gesellschaftlichen Bereichen Gesundheit und Arbeit das vormals alternativlos erscheinende Vertrauen in die Expertisen fachlicher Autoritäten und gesellschaftlicher Wissensreservoirs wie z.B. Bevölkerungsstatistiken heraus, stellt ihnen das individuelle Wissen der Selbstvermessung entgegen und wendet es letztlich nach ganz ähnlichen Prinzipien auf sich selbst an. Diese Untersuchung widmet sich daher dezidiert der Frage in welcher Weise Selbstbestimmung, (Selbst-)Disziplinierung und biopolitische Regulierung im Diskurs der Selbstvermessung miteinander verschmolzen sind. 



\section{Das Forschungsprogramm}

Nach einer Beschreibung des methodischen Aufbaus der Arbeit, und der epistemologischen Grundprämissen (Abschnitt II. Iff.), die mit einer Verhandlung der Konzepte »Ursprung « und »Herkunft« schließt (Abschnitt II. 2.4), beginnt die Analyse bei der Beschreibung von Ursprungsnarrativen, die innerhalb des Diskurses selbst verhandelt werden (Abschnitt II. 2.5).

Abseits der dort vorherrschenden Suche nach genuinen ersten Self-Tracker*innen, wird das Aufkommen von Selbstvermessungstechnologien hier wissenshistorisch im Zusammenhang mit der wachsenden Bedeutung von numerischem Wissen im Allgemeinen betrachtet. Den im Diskurs überwiegenden Versuchen eine Ursprungsgeschichte der Self-Tracking-Technologien zu zeichnen, stellt dieser genealogische Exkurs die Herleitung des quantifizierten Selbst gegenüber, das sich zunächst unabhängig von Self-Tracking-Technologien, parallel mit statistischen Fremd- und Selbstbeschreibungsformen der Gesellschaft entwickelt (Abschnitt III. Iff.).

Der Abschnitt folgt der Annahme, dass sich der Wandel von Vermessungsformen in weiten Teilen äquivalent zu einem Wandel entsprechender Subjektivationsformen vollzieht. Während die biopolitische und zentralistische Vermessung des Individuums im Zusammenhang mit seiner Disziplinierung auftritt, sind mit der Veröffentlichung von Statistiken, also der medialen Verbreitung numerischer Wissensformen, zunehmend Selbstregulationsprozesse zu verzeichnen. In Ergänzung zu diesen idealtypisch unterschiedenen Verdatungsphasen wird im Zuge der vorliegenden Analyse nun eine dritte Verdatungsphase ergänzt, in der sich neben der regulativen Subjektivation anhand von statistischen Daten über Individuen nun auch eine Dezentralisierung der Erhebungs- und Analysemittel derartiger numerischer Wissensbestände vollzieht.

Die hieran anschließenden Abschnitte untersuchen diese Dezentralisierungsprozesse dann genauer auf der Ebene des sich um die Entwicklung von Self-Tracking-Technologie herausbildenden Diskurses und seiner Schnittmengen mit vorgängigen und angrenzenden (Teil-)Diskursen. 
So geht Abschnitt III. 2 zunächst der Frage nach, warum der Begriff des SelfTracking ausgerechnet in den wissensintensiven Arbeits- und Konsumtionsmilieus westlicher Postindustrienationen das erste Mal formuliert wird.

Trotzdem sich der Self-Tracking-Diskurs in einem weltumspannenden Netzwerk aus Medientechnologien entfaltet, ist sehr auffällig, dass er thematisch nicht selten lokalspezifische Probleme westlicher Technologiemetropolen behandelt. Es sind auch die hier angesiedelten Tech-Communities, die die Infrastruktur regionaler Diskursveranstaltungen wie Konferenzen und kleinerer Talks gewährleisten.

$\mathrm{Zu}$ Beginn wird der Diskurs auf Zusammenhänge zwischen Innovationsprozessen und regionale (Arbeits)kulturen sowie Wirtschaftsstrukturen befragt, denn der Self-Tracking-Diskurs ist über viele Elemente, wie ökonomische Prekarisierung, Individualismus, Unkonventionalität, allgemeine entrepreneuriale Kultur und technologieutopistische Problemlösungskreativität, mit anderen Diskursen verbunden. Im Detail wird hier dann nach dem Einfluss gefragt, den die technologiepolitische Regionalentwicklung auf die Diskursorganisation und die Entstehung von Self-Tracking-Technologien ausübt. Anstatt Self-Tracking-Technologien aber als das direkte Ergebnis lokaler Förderprogramme zu beschreiben, werden vielmehr Top-Down-Perspektiven (wie gouvernementale Steuerungsbemühungen der lokalen Wirtschaftspolitik) mit der Bottom-Up-Perspektive auf die TechCommunities vermittelt.

Zusammengenommen dienen die hier freigelegten interdiskursiven Zusammenhänge als ein erster Hinweisgeber auf die spezifische Subjektivität, die als Ursprung und Ziel die Entwicklung von Self-Tracking-Technologien anleitet.

Diese Subjektivität ist dabei durchaus als ein Ausdruck von gesellschaftlichen Leitbildern wie z.B. der kreativen Unternehmerin und wirtschaftspolitisch forcierten Subjektivierungsprogrammen anzusehen, sie ist aber nicht mit ihnen gleichzusetzen. Sinnbildlich für die produktiven Elemente des Foucault'schen Machtkonzeptes zeigt sich in diesem Teildiskurs vielmehr ein Subjekt, das die fordernden und fördernden Agenturen inzwischen selbst durch animierende Parolen zur Herstellung der Bedingungen bewegen will, die für die Entwicklung von kreativen Projekten und Unternehmen nötig sind.

Obgleich sich die jüngere Geschichte der Verdatung, die auch die Vorgeschichte der Selbstverdatung darstellt, eng mit der Entstehung ökonomischer Prozesse vollzieht und zuallererst mit Rationalisierung assoziiert wird, lassen sich die visuellen, numerischen und sprachlichen Artefakte, die durch die Quantified-SelfCommunity produziert werden, nicht mit puritanischer, unternehmerischer Rationalität gleichsetzen. Sie zeigen vielmehr deutlich, dass die Selbstverhältnisse, die im Zuge der Selbstverdatung angestrebt werden, auch eine Orientierung am libidinös besetzten Ideal der kreativen Künstlerin beinhalten. Kongruent zu der anhaltenden Emotionalisierung moderner Arbeitsformen und der mit ihnen verbundenen Symboliken und Semantiken, lässt sich vielmehr auch ein kreatives Spiel 
mit den numerischen Erzeugnissen aus Selbstverdatungsverfahren verzeichnen, durch das viele Self-Tracker*innen zwischen ästhetischen Ausdrucksformen und einer technologisch verwirklichten Selbststrenge changieren. Einige der vielen Varianten kreativer Umgangsformen mit Daten, die sich von der vergleichsweise variationsarmen Ästhetik des Rechnungswesen oder sozialwissenschaftlicher Statistiken abgrenzen, ohne dabei aber die durch sie verhandelten Themen wie z.B. Leistung oder belastungsbedingte Gesundheitsschäden zu verlieren, werden daher unter Abschnitt III. 2. dargestellt. Sie werden dabei als ein Ausdruck der enthusiastischen Affizierung technokratischer Arbeitsprozesse durch ein Subjekt beschrieben, das mitunter vom stereotypen Modell künstlerischer, nichtentfremdeter, expressiver Arbeit angeleitet ist. Als Extrembeispiele dienen sie dazu aufzuzeigen, wie die Organisatorin oder Verwalterin ihrer Selbst im Diskurs des Self-Tracking als nonkonforme Kreativunternehmerin reinkarniert.

Der darauffolgende Abschnitt (III. 2.5ff.) leitet zu einer Analyse von Machtfragen innerhalb des Diskurses über. Es zeigt sich dabei deutlich, dass der organisierte Teil des Self-Tracking-Diskurses den Entwicklungen in verwandten DatenDiskursen aufmerksam folgt und mit eigenen Mitteln an diskursiven Verschiebungen oder Akzentuierungen arbeitet.

Durch die Betrachtung von naturalisierenden Metaphern wird hier herausgestellt, wie Teile der Quantified-Self-Community anhand von Diskursinterventionen charakteristische Begriffe und Sprachbilder aus dem angrenzenden Big-Data-Diskurs aufnehmen und nach eigenen Prämissen politisieren. Die fortschrittsenthusiastische Progressivität der Community wendet sich dabei gleichermaßen gegen die Datenökonomie als auch gegen passive DatenschutzArgumentationen, denen sie den Wunsch nach mehr Kontrolle über die eigene Verdatung entgegensetzen. Hier wird das erste Mal die kummulative Logik des Self-Tracking-Diskurses deutlich, dernach mehr Daten über sich selbst zu besseren Möglichkeiten der Selbstbestimmung führt.

Der immanente Zusammenhang den Subjektkonzepte und Daten innerhalb des Diskurses ausbilden wird unter Abschnitt III. 3 aufgefechert. Hier werden drei diskursbestimmende Selbstkonzepte differenziert und beschrieben, die jeweils mehr Gewichtung auf Körperfunktionen und Emotionen (3.1), das Selbst im zeitlichen Verlauf (3.2) oder Routinen (3.3) legen. Der gegen Ende des 2. Abschnitts begonnene Bezug zur Großdatenforschung wird in diesem Abschnitt zudem weiter mitgeführt und mit Blick auf epistemologische Verwandtschaften zwischen SelfTracking- und Big-Data-Diskurs verengt.

Obgleich der Begriff der Optimierung innerhalb des Diskurses kaum verwendet wird, wird er medial besonders häufig mit dem Phänomen des Self-Tracking in Verbindung gebracht. Der vierte Abschnitt widmet sich daher zunächst der Systematisierung von Optimierungs- und Selbstoptimierungsbegriffen im Zeichen des jüngeren (kultur)geschichtlichen Wandels von Arbeit und setzt beide in Beziehung 
zu den Unabhängigkeitsambitionen der Quantified-Self-Community. Dabei wird deutlich, dass Arbeits- und Leistungsoptimierung und die Erlangung von Selbstbestimmtheit im Diskurs des Self-Tracking nicht als Gegensätze erscheinen. Das Hauptaugenmerk dieses Abschnitts liegt daher darauf, die Modi herauszustellen, durch die sich Selbstoptimierung und die Politisierung von Wissen im Diskurs verschränken. Der Optimierungsbegriff, so wie er seit seiner Genese während der Rationalisierungsbewegung der 1920er Jahre existiert, wird dazu auf der Ebene seiner Prämissen mit den Zielen populärer Selbstvermessungsprojekte verglichen. Wie sich zeigt, lassen sich weder die Optimierungsbegriffe der tayloristischen Betriebswissenschaften, noch die Begriffe der quantitativen Selbstoptimierung mit einer grenzenlosen Steigerung gleichsetzen. Vielmehr stellt in beiden Fällen das angestrebte Optimum ein Leistungsniveau dar, das sich über längere Zeit einhalten lässt. Parallelen bestehen zudem darin, dass in beiden Fällen versucht wird Arbeitsleistung und Gesundheit in ein gemeinsames numerisches Verhältnis setzen.

Zusammengenommen scheinen sich über numerische Selbstvermessungen Optimierungs- und auch Disziplinierungstechniken in moderne Kreativberufe zu übertragen, die ihre Kontur eigentlich über die Abgrenzung zu den rigiden Organisationsprinzipien der tayloristisch-fordistischen Arbeitswelt erlangen. Anders als in den industriegesellschaftlichen Arbeitsbegriffen zielen die Selbstvermessungstechniken aber nicht nur auf die Optimierung des Zusammenspiels zwischen Mensch und Maschine in der Produktion, sondern sind in viel generellerer Weise darauf ausgerichtet, die Produktionsbedingungen als Ganze zu optimieren. In Arbeitsfeldern in denen Arbeit und Selbst zu kongruenten Kategorien werden, meint dies im buchstäblichen Sinne Selbstoptimierung.

Insbesondere das Self-Tracking im Home Office zielt auf eine Subjektivität, die sich gegen die emotionalen Hemmnisse und disziplinarischen Zurichtungen des maschinistischen Betriebskapitalismus richtet und dabei ihre elementaren Prämissen enthusiastisch affiziert, indem sie elaborierte Problemlösungsfähigkeiten innerhalb eines zur Norm erhobenen Klimas der Kreativität und der Eigenständigkeit durch die Erfindung von Selbstoptimierungs- und Selbstdisziplinierungstechnologien unter Beweis gestellt werden.

Die Unabhängigkeits- und Selbsterkenntnisrhetorik, unter deren Vorzeichen derartige Technologien entwickelt werden, versinnbildlicht dabei aus einer Foucault'schen Perspektive sehr treffend das ambivalente Verhältnis zwischen verringerter Fremdbestimmung und einer hierdurch ins Werk gesetzten eigenverantwortlichen Steuerung, die als disziplinarische Kontrolle zweiten Grades durch die Kontrollierten selbst ausgeübt wird.

Dabei richtet sich das Selbstbestimmungsprojekt des Self-Tracking als praktische Form der Kritik gegen den Widerspruch des allgegenwärtigen Ansporns zu mehr Selbstständigkeit im Zeichen der Individualisierung von Risiken und Verant- 
wortung bei gleichzeitigem Ausbleiben einer Individualisierung der gesundheitlichen und wirtschaftlichen Bemessungsgrundlagen.

Denn die gleichen Agenturen, die das Individuum zu mehr Selbstkontrolle animieren, können selbst nur über Bevölkerungsdurchschnitte Auskunft geben und bieten damit Informationen an, die im Mikrokosmos der Freelancer*innen und ihren hochspezifischen Problemen häufig kaum mehr einen Nutzen haben. Dabei wird das Self-Tracking hier erstmals als eine Strategie deutlich, um von der Rezeption kollektiver Vergleiche zur individuellen Herausforderung dieser Vergleichsbasis zu gelangen. Das Quantifizierte Selbst sieht sich damit in den Aggregaten der Bevölkerungsstatistik zwar nicht genügend repräsentiert, strebt ansonsten allerdings vor allem danach, die Praktiken und Wissensformen der biopolitischen Agenturen auf sich selbst anzuwenden.

Wie sich in Abschnitt 5 zeigt, stößt der kompromisslose Individualismus der Quantified-Self-Community bei der Bemessung von Leistung und Gesundheit aber auch schnell an seine Grenzen, da es sich bei diesen Scores um relationale Einheiten handelt, deren Nutzen sich erst im überindividuellen Vergleich einstellt. Umgekehrt lässt sich sagen, dass sich die (Re)integration von überindividuellen Vergleichswerten im Self-Tracking-Diskurs parallel zu einer steigenden Popularität des Self-Tracking allgemein und der Verbreitung kommerzieller Self-TrackingTechnologien vollzieht, die durch ein hohes Maß an Standardisierung und zentrale Infrastrukturen zumindest annäherungsweise die Orientierungsfunktionen von massenmedial distribuierten Statistiken übernehmen. Anders als in Relation zu den enorm abstrakten Ausschnitten großer Sozial-Panels, so wie sie in den Massenmedien dargestellt werden, sind die Konsument"innen der kollektiven Durchschnitte verschiedener Selbstvermessungstechnologien aber auch unmittelbar ihre Produzent"innen. Zudem können Strategien des Einwirkens auf sich selbst hier unmittelbar Feedback-gestützt getestet und verändert werden, was sie in noch direkterer Weise zu Subjektivationstechnologien macht. Bezogen auf die unter Abschnitt III. 1 vorgenommene, idealtypische Trennung numerischer Selbst- und Fremdbeschreibungsformen im jüngeren historischen Verlauf, lässt sich sagen, dass sich hier die Kernfunktionen der unter III. 1.2 und III. 1.3 beschriebenen Verdatungsphasen verbinden - d.h. die dezentrale Entwicklung von Selbstvermessungstechnologien einerseits und die Reflexivität öffentlich einsehbarer Statistiken andererseits.

Der Abschnitt III. 5.2 führt die Untersuchung von Selbstverdatungsprojekten im Zusammenhang mit postmodernen Arbeitsfeldern und beruflicher Alleinselbstständigkeit weiter, rückt dabei aber zunehmend von der engen Fokussierung auf die Technologieenthusiasten und die experimentellen Prototypen der SelfTracker"innen ab und betrachtet kommerzielle Verdatungssysteme, die teils von hunderttausenden Nutzer*innen für die Eruierung von Leistung und Gesundheit verwendet werden. 
Daten dieser Art bieten zudem die Möglichkeit von einer indirekten Ressource (der Leistungssteigerung) zu einer Ressource zu werden, die direkt in Wert gesetzt oder zur Erhöhung von sozialem Prestige eingesetzt werden kann. Die subjekttheoretisch apostrophierte Selbstvermesserin kann sich als kalkulatorisch versierte Unternehmerin ihrer Selbst nicht nur auf ihre eigene Vermessungshistorie berufen (z.B. um qua Training und organisatorischer Eingriffe die eigene Leistungsfähigkeit zu verbessern), sie ist durch die Daten auch in der Lage ihre Leistungsfähigkeit unter Beweis zu stellen und sich in bestimmten Kontexten als funktionale und berechenbare Arbeitskraft oder als gesunder und körperbewusster Mensch zu konstituieren.

Der sechste Abschnitt befasst sich noch eingehender mit der Kommerzialisierung und Verbreitung von Self-Tracking-Technologien und zeichnet den Weg nach über den Praktiken der Selbstkontrolle und zugehörige Bottom-Up-Innovationen in vergleichsweise konventionelle Kontrollformen von Angestelltenverhältnissen und des Gesundheitswesens integriert werden.

Interdiskursive Beziehungen zwischen der dezentralen Entwicklung von SelfTracking-Technololgien und dem Personalmanagement lassen hier den Schluss zu, dass sich Unternehmen und Selbstunternehmen nicht nur mit vergleichbaren Problemen konfrontiert sehen, sondern auch nach ähnlichen Lösungswegen suchen, um sie zu kontrollieren.

Unter der Beschränkung auf spezifische Teilbereiche der betrieblichen Personalentwicklung und der progressiven Selbstverdatung offenbart sich hier ein diskursiver Zirkelschluss: Während die technologieaffinen Freelancer selbstständig Applikationen für das Home Office entwerfen, die eine auffällige Nähe $\mathrm{zu}$ den Zeiterfassungssystemen der Großraumbüros aufweisen, orientiert sich nun das in diesen Kreisen vielfach als restriktiv und autoritär angesehene Angestelltenverhältnis an den Überwachungs- und Disziplinartechnologien des unabhängigen Selbstunternehmens. Ein großer Teil der Akzeptabilität betrieblicher Vermessungs-Technologien und -Praktiken begründet sich vielmehr gerade darin, dass technische Verfahren für die Protokollierung und Analyse von Leistung und Gesundheit in den letzten Jahren vor allem außerhalb der Betriebe oder des institutionalisierten Gesundheitsmanagements in den Innovationsnetzen technologieaffiner, emanzipatorischer Subkulturen und Startup-Szenen diskutiert, entwickelt und zur Marktreife gebracht wurden.

Vergleichbar mit dem Mittelwerten überindividueller Selbstverdatungssysteme für das Home Office operationalisieren diese Technologien hier nun in Bezug auf gesundheitliche Präventionskategorien den Anschluss an spezifische Nutzer*innengruppen, Unternehmensbelegschaften oder einzelne Abteilungen. Analog lässt sich auch die Verbindung von individuellem Tracking mit kompetitiven Datenvergleichen innerhalb der Belegschaft als ein neuer Modus deuten, in dem innerbetriebliche Konkurrenz als strukturierendes Organisationsprinzip der Unterneh- 
mensfitness aktiviert wird. Über die technologische Verschaltung der Vitaldaten von Angestellten zu numerischen Kollektiven und offen einsehbaren Durchschnitten wird so eine kompetitive Selbstsorgekultur etabliert, die das Spannungsproblem zwischen maximaler Arbeitsleistung und kosteneffizienter Erhaltung der Arbeitsfähigkeit approximativ als Teil einer modernen Personalentwicklung zu lösen versucht.

Die kalkulatorische Logik und der Datenhunger der hieraus entstehenden selbsttragenden Kontrollsysteme machen allerdings bei den Angestellten entsprechender Unternehmen keinen Halt. Unter Abschnitt III. 6.5 werden die zur Zeit generellsten Entwicklungen skizziert, die aktuell aus der Interdiskursbeziehung zwischen Self-Tracking und Gesundheitswesen hervorgehen: So bieten immer mehr global agierende private Versicherungsdienstleister und gesetzliche Krankenkassen spezielle Bonustarife im Zusammenhang mit Self-TrackingTechnologien an, um Klient*innen ein direktes Beobachten und Antizipieren der positiven und negativen Effekte von Verhaltensmustern $\mathrm{zu}$ ermöglichen. Die hier vorherrschenden Gefahrenszenarien setzen meist bei sehr allgemeinen Bildern an, die auch über den speziellen Kontext der Arbeitsüberlastung hinaus anschlussfähig sind, dabei allerdings ihre Verbindung zu den Symptomatiken halten, die als spezifische Probleme der postindustriellen Wissensgesellschaft gelten: Allen voran Stress, Bewegungsmangel und schlechte Ernährung. Trotzdem diese Aussagen leicht in Zweifel gezogen werden können, tragen sie doch zu einer diskursiven Sedimentierung einer monokausalen Ursacheninterpretation von im Grunde vielen verschieden und sehr komplexen Krankheiten bei, durch die die Verantwortung erneut individualisiert und Handlungsoptionen noch enger an technologische Lösungen gekoppelt werden.

Durch die Integration von Activity-Trackern in die Berechnung von Versicherungstarifen integriert sich damit auch die inhärente Logik der Versicherungen schrittweise in alltägliche Tagesabläufe. Im Spiegel numerischer Vergleiche werden Tätigkeiten, denen bisher kaum Aufmerksamkeit zugekommen ist, so zu einer potentiellen Ressource im Gesundheitswettbewerb. Mehr noch als im Zusammenhang mit den Wellness-Programmen des betrieblichen Gesundheitsmanagements kann so zukünftig jeder Alltagsaspekt in den Inklusionssog datengetragener Versicherungsmodelle geraten - wodurch sich auch die kompetitive Grundierung der vergleichenden Selbstvermessung potentiell immer weiter mit unterschiedlichen Lebensbereichen verzweigt. Und das meint in erster Linie die Forcierung von gesundheitsökonomischen Konkurrenzbeziehungen innerhalb von Kohorten, Unternehmensbelegschaften oder Familien. Als das Ergebnis diskursiven Drängens breitet sich die quantifizierte Selbstwahrnehmung so nach und nach in viele Bereich des gesellschaftlichen Lebens aus, die noch vor kurzem von der numerische Sprache und den kalkulativen Logiken der Verdatung unberührt waren. Das quantifizierte Selbst ist auf dem Vormarsch. 



\section{Methodisches Vorgehen}





\title{
1 Die Beschreibung progressiver Selbstverdatung Self-Tracking im Schnittfeld von Diskursanalyse und Gouvernementalitätsstudien
}

\author{
»From Foucault's perspective, >Foucault< is \\ not a valid argument for a particular analy- \\ tical strategy.« \\ Niels Åkerstrøm Andersen
}

\subsection{Die Unordnung der Diskursforschung}

Seit einigen Jahren sind unter den zahllosen Auseinandersetzungen mit dem vielschichtigen und diskontinuierlichen Werk Foucaults zunehmend Anschlussarbeiten zu verzeichnen, die von hermeneutischen Primärtextexegesen und der Suche nach dem »wahren « Foucault ablassen und »Foucault « in erster Linie als Anregung für pragmatische Konzeptionen von Einzelfallstudien nutzen (Keller 2004: 7ff.). Das unübersichtliche Spektrum an Anschlüssen verzweigt sich vor allem entlang von drei dominanten Differenzierungslinien. Erstens besetzen Kultur- und Sozialwissenschaften, Pädagogik und Geschichtswissenschaften sowie ihre jeweiligen Bindestrichdisziplinen einzelne Begriffe und Gegenstände in Foucaults Werk nach eigenen fächerspezifischen Prämissen. Wobei sie zweitens auf verschiedene Epistemologien und Wissenschaftstheorien rekurrieren. Die unterschiedlichen Akzentuierungen der einzelnen Werkphasen bilden schließlich die dritte Differenzierungsdimension.

Insbesondere politikwissenschaftliche aber auch phänomenologisch-wissenssoziologisch geprägte Diskursforschungen stützen sich in ihren Analysen auf klar abgrenzbare, institutionell verengte Konzeptionen von Diskurs, deren Einheit als deckungsgleich mit z.B. politischen Debatten im Bundestag (vgl. Schwap-Trapp 2006), wissenschaftlichen Spezialdiskursen (vgl. Waldschmidt 2004) oder massenmedial getragenen Prozessen der öffentlichen Meinungsbildung erscheinen (vgl. Mattissek 2010). Charakteristisch für Studien dieses Typs ist die klare Trennung zwischen Theorie und Methode. D.h. sie verbinden entweder das zumindest in Teilen ausgearbeitete Instrumentarium der Archäologie des Wissens (Foucault 1981[1969]) mit sozialwissenschaftlichen Theorien oder die bei Foucault angeleg- 
ten Theorien mit den jeweils geltenden Methodenstandards einer Inhalts- oder Deutungsmusteranalyse, der interviewbasierten Biografieforschung oder der ethnomethodologischen Konversationsanalyse $\mathrm{zu}$ einem klassischen, dualistischen Forschungsaufbau.

Dies mag nicht zuletzt auch damit zusammenhängen, dass Foucault sich selbst keiner Disziplin eindeutig zugeordnet hat und es ebenfalls ablehnte eine eigene Forschungstradition zu begründen (Andersen 2003: 115). Darüber hinaus verfolgte Foucault wechselnde Erkenntnisinteressen, für die er jeweils abgewandelte analytische Instrumentarien verwendete ${ }^{1}$ und dessen methodische Reflexion über die »Archäologie« hinaus weitestgehend ausblieb.

Das Ergebnis dieser Entwicklung ist ein über Jahrzehnte gewachsenes, diverses Feld der Diskursforschung, in dem unterschiedliche Disziplinen und Teildisziplinen mit unterschiedlichen Schwerpunkt- und Zielsetzungen vertreten sind, welche die Grundzüge der Foucault'schen Diskurstheorie mit anderen Theorie- und Methodenbausteinen kombinieren, rekombinieren oder kontrastieren. Ein voraussetzungsloses Anknüpfen an den Begriff des »Diskurses« scheint angesichts dieser Unordnung der Diskursforschung kaum möglich.

Spätestens seit der postumen Veröffentlichung der Vorlesungen »Sicherheit, Territorium und Bevölkerung« (2004[1977-1978]) sowie »Geburt der Biopolitik« (2004[1978-1979]) wird die Diskursanalyse im deutschsprachigen Raum zudem durch das vergleichsweise junge Forschungsprogramm der Gouvernementalitätsstudien erweitert.

In den folgenden Abschnitten sollen verschiedene Prämissen der Gouvernementalitätsstudien sowie der Diskurstheorie auf der Ebene ihres jeweiligen Erkenntnisinteresses und der Methoden, die sie dafür aufwenden miteinander verhandelt werden. Der Vergleich beider Ansätze wird dabei durch die Frage angeleitet, wie sich eine Untersuchung der konstitutiven Bedingungen von Selbstvermessungstechnologien als Technologien des Selbst theoretisch und forschungspragmatisch positionieren muss, um das wechselseitige Verschränkungsverhältnis freiwilliger, progressiver Nutzung und planvoller Etablierung von Selbstvermessungstechnologien in den Blick zu bekommen.

\subsection{Zum Verhältnis von Gouvernementalitätsstudien und Diskursforschung}

Eines der zentralen, d.h. über die unterschiedlichen Werksepisoden fortbestehenden Forschungsinteressen Foucault's betrifft die Suche nach den Mechanismen der

1 »Ich habe keine Methode, die ich unterschiedslos auf verschiedene Bereiche anwende« (Foucault 2003[1976-1979]: 521f.). 
Herstellung gültigen Wissens, sowie der hierauf begründeten Ordnungen und Subjektformierungen. Es ist insofern eine Suche nach den kommunikativen Bedingungen der Erzeugung sozialer Wirklichkeit, dessen Kulminationspunkt der Diskurs bildet. Foucault hat diesen Begriff dabei bekanntermaßen unterschiedlich akzentuiert: Die Foucault'sche Diskurstheorie kennt sowohl einen frühen, vornehmlich strukturalen Diskursbegriff, der induktiv als Muster von Regelmäßigkeiten aus der Analyse humanwissenschaftlicher Verifikationsverfahren hervorgegangen ist, als auch einen Begriff, der die ursprüngliche Trennung von diskursiven und nichtdiskursiven Praktiken (vgl. Foucault 1981: 234) aufhebt und Diskurse stärker in Beziehung zu Machtprozessen setzt. Anhand des genealogischen Verfahrens dekonstruiert eine so angelegte Diskursanalyse transzendentale Subjektverständnisse als historisch bedingt und nimmt vor allem all jenes in den Blick, was nicht gesagt oder getan werden kann.

Mit den Vorlesungen zur Gouvernementalität werden die Untersuchungen zur Verschränkung von Wissen und Macht im Prozess der Wahrheitsproduktion schließlich mit Blick auf staatliche und nicht-staatliche Formen der Macht weiter differenziert: »In seinen Analysen zu (neuen) Formen des Regierens als politischer Steuerung menschlichen Verhaltens nimmt Foucault verstärkt auch Praktiken und Technologien des Selbst in den Blick, sodass die Gouvernementalitätsperspektive als Scharnier im Dreieck von Macht, Wissen und Subjektivität operiert « (Angermüller, van Dyk 2010: 9). Die Beschreibung der diskursiven Sinn- und Wissensproduktion tritt hier gegenüber der Beschreibung nicht-diskursiver Praktiken der Fremd- und Selbststeuerung in den Hintergrund, wobei allerdings eine Reflexion dieser Schwerpunktverschiebung oder eine Erläuterung des Verhältnisses zwischen den Grundrissen der Gouvernementalitätsheorie und der Diskurstheorie sowie der Überschneidung ihrer Erklärungsansprüche unterbleibt. ${ }^{2}$

Die jüngeren an Foucault angelehnten Gouvernementalitätsstudien, haben diesen offenen Deutungsraum inzwischen dadurch besetzt, dass sie die Grundrisse der Gouvernementalitätsheorie mit methodisch bewusst puristisch angelegten Zeitdiagnosen zu einem Forschungszweig verwoben haben, der nach systematischen Verbindungen zwischen Rationalitätsformen, Regierungsweisen und Selbstverständnissen sucht. Derart konzipierte Gouvernementalitätsstudien fokussieren vor allem auf technologische Regierungsaspekte. Dies umfasst Machtund Selbsttechnologien ebenso wie Arrangements aus medialen Netzwerken, Apparaten, Dokumentations- und Visualisierungssystemen sowie administrativen Instrumenten, die in Form von Datenevaluation, Datenanalyse, Reglementierungen oder vertraglichen Vereinbarungen Subjekte zum Ziel disziplinierender, normalisierender, ermächtigender oder präventiver Verfahren machen (vgl. Bröckling 2010: 26ff.). 
Eine solche Forschungsperspektive bietet vielversprechende Anschlussmöglichkeiten für die Analyse des Selbstvermessungsphänomens; allerdings ist fraglich, ob ihr enger empirischer Zuschnitt ausreicht um den progressiven Aspekt der Selbstvermessung, als Untersuchung eines in der kontinuierlichen Entwicklung befindlichen Feldes, ergebnisoffen zu untersuchen.

Denn eine so gewendete Forschungsperspektive unternimmt zwei prägnante Schwerpunktsetzungen: Zum einen untersucht sie Wissensformen und Rationalisierungen im Kontext spezifischer Problemdiagnosen oder Notstände und zum anderen die mit ihnen korrespondierenden Strategien für die Bewältigung der diagnostizierten Probleme (vgl. Bröckling 2010: 24). ${ }^{3}$ Dabei geht sie davon aus, dass Regime des Regierens oder Selbstregierens mehr oder weniger ausgearbeiteten, aber meist schriftlich niedergelegten Programmen folgen oder sich planvollen, meist wissenschaftlich gestützten Verfahren bedienen, um vorab definierte Ziele zu erreichen (vgl. Bröckling 2010: 36). Ihren empirischen Ausgangspunkt bilden daher vor allem Manuale, Handbücher, Leitfäden oder Richtlinien, die praktisches Wissen in Form von Empfehlungen, Anleitungen oder Anweisungen beinhalten und in der Regel eine klar benennbare Urheberin aufweisen.

Aus diskurstheoretischer Sicht stellt eine Beschränkung auf Manuale, Anweisungen oder Pläne allerdings bereits einen künstlich gewählten Ausschnitt inmitten diskursiver Prozesse dar, von dem aus die Erscheinungsbedingung bestimmter Aussagen, Strategien und Gegenstände zwangsläufig unterbelichtet bleiben. Damit erscheint "Gouvernementalität« aus Sicht der diskursanalytischen Methodologie eher als eine Fragestellung, denn als ein vollständiges Forschungsprogramm.

Eine derart auf planvolles Einwirken ausgerichtete Forschungsperspektive läuft zudem Gefahr, in das Fahrwasser kausaler oder intentionalistischer Erklärungen $\mathrm{zu}$ geraten, denen zufolge Strategien und Pläne als Operationalisierungen eines Willens erscheinen.

Von den methodologischen und methodischen Problemen, die derartige $\mathrm{Zu}$ gänge mit sich bringen, abgesehen, mögen Intentionen der Ausgangspunkt eines Ereignisses sein; sie sind aber nicht identisch mit den Bedingungen seines Erscheinens. Der diskursanalytische Ansatz begründet sich demgegenüber geradezu darauf »dem Subjekt seine Rolle als ursprüngliche Begründung zu nehmen und es als Variable und komplexe Funktion des Diskurses zu analysieren « (Foucault 2001a[1969]: 1029). ${ }^{4}$ Macht-Wissen-Komplexe lassen sich besonders dadurch freilegen, dass in der Analyse die Aufmerksamkeit auf die Differenz zwischen potentiell möglichen Aussagen und Handlungen und den tatsächlichen Aussagen und Souveränität generiert, die Foucault im Jahre 1973 an der Katholischen Universität in Rio de Janeiro gehalten hat (Foucault 2002: 10). 
Handlungen gerichtet wird, die sich in diskursiven Prozessen durchsetzen. Entsprechend lässt sich der Sinn oder die Bedeutung von Aussagen nicht auf die Absicht oder die Gedanken eines Subjekts zurückführen (vgl. Foucault 1981: 182).

Subjektkonstitutionen sind nicht als Ergebnis einer sich durchsetzenden Intentionalität, einer planvollen Selbst- oder Fremdeinwirkung zu betrachten; vielmehr stellen sie als Effekt diskursiver Prozesse Idealvorstellungen dar, die zum Ziel von Bestrebungen werden oder an denen sich Selbst- und Fremdbeschreibungen ausrichten können. Zu Subjektivierungsprogrammen werden sie dann, wenn derartige Selbstkonstitutionen durch öffentliche Konventionen begrenzt oder ermuntert werden.

Wie Bröckling und Krasmann selbst schreiben, neigen viele Gouvernementalitätsstudien daher dazu sich im »Zirkel der Selbstbestätigungen« zu verfangen und nur herauszufinden, was sie bereits wissen (Bröckling 2010: 39). Das betrifft eng fokussierte Einzeluntersuchungen, die verschiedenste Phänomene als Effekte der immer gleichen Strategien und Rationalitäten subsumieren ebenso wie historische Großnarrative, welche die Entwicklung von Regierungsweisen mit einer evolutionären Logik vom Liberalismus bis zum Neoliberalismus nachzeichnen. Unter vielen Gouvernementalitätsstudien hat dies zu einem impliziten Finalismus geführt, der eine kontinuierliche Rationalisierung und Optimierung unterstellt (vgl. Bröckling 2010: 33f.) und durch die argumentative Reproduktion von Sachzwanglogiken $\mathrm{zu}$ einer wissenschaftlichen Fundierung der beschriebenen Regierungsweisen im Sinne eines Unvermeidbarkeitsnachweises beiträgt (vgl. Lemke 2007: 60f.).

Dies mag auch damit zusammenhängen, dass in der Fokussierung der Gouvernementalitätsstudien auf einen eindimensionalen Materialtyp bei gleichzeitiger hypothetischer Gleichsetzung von Regierungsprogrammen mit den dazugehörigen Blaupausen, eine kausale Argumentationsweise immer schon latent angelegt ist. Die sich wechselseitig bedingenden, verstärkenden oder diametral entgegenlaufenden Kräfteverhältnisse, die im Zuge der Konstituierung und Implementierung von Regierungsweisen auch als Diskontinuitäten, Brüche, Irritationen und Abwandlung wirksam werden können, werden so häufig durch eine lineare Argumentation überformt.

Eine Analyse, die darauf abzielt zu beschreiben, wie Machttechnologien als Technologien des Selbst diskursiv hergestellt werden, muss über die Frage hinausgehen, welche Ziele mit der Installation von Regierungsweisen verbunden werden und ihren Fokus über einzelne Textgattungen oder die durch sie beschriebenen, angeleiteten oder legitimierten Technologien hinaus ausdehnen. Sie gewinnt ihre Erklärungskraft vielmehr erst indem sie die Relationen zwischen verschiedenen Texten in den Blick nimmt und Aussagen, sowie Diskurspositionen, Ziele, Strategien und vorläufige Ergebnisse in ihrer wechselseitigen Abhängigkeit rekonstruiert. Dies betrifft vor allem Relationen mit drei verschiedenen Verweisungsrichtungen: (a) Zum einen die diskurskonstituierenden Relationen des Kerndiskurses, (b) die 
Interdiskursrelationen, die den Kerndiskurs mit Teildiskursen der Massenmedien oder institutionalisierten Diskursinterventionen z.B. des Gesundheitsmanagements, des Marketings der Konsumgüterindustrie oder der Wissenschaft in Beziehung bringen, (c) als auch die Relationen zwischen Diskurselementen im zeitlichen Verlauf, die anzeigen, an welche Wissenstraditionen angeschlossen und mit welchen Kontinuitäten gebrochen wird. Denn erst durch einen solchen zumindest flüchtigen Blick auf Vorläuferentwicklungen, lassen sich die gegenwärtigen Selbstverständnisse und Identitätsfiktionen, die im Diskurs als Ursprung oder Motiv von Selbstvermessung auftauchen, als eine von den intentionalistischen Selbstauskünften der Beteiligten losgelöste Herkunftsgeschichte ${ }^{5}$ beschreiben $^{6}$. Zusammengefasst ermöglichen es diese Relationen ein Verständnis von den Bedingungen zu erlangen, unter denen bestimmte Technologien populär werden können, bestimmte Bereiche des Lebens von ihnen durchdrungen werden, wobei andere unberührt bleiben und schließlich ein Verständnis davon, welche Kriterien bei der Entwicklung von Selbstvermessungstechnologien berücksichtigt werden müssen, damit sie Legitimität, Wahrheit, Kreativität oder Wissenschaftlichkeit für sich beanspruchen können.

Das Feld der Selbstvermessung ist in besonderem Maße dazu geeignet nachzuzeichnen, wie eine bestimmte Form der Subjektivität aus dem Spannungsgefüge verschiedener Deutungskonflikte und Macht-Wissen-Komplexe hervorgeht, da ihre Konstitutionsbedingungen in umfassender Weise offen liegen. Numerische Daten sowie Taxonomien und Klassifikationssysteme werden ebenso wie ihre sprachlichen Erläuterungen, Bedarfsbeschreibungen, Kommentare, Kritiken und Gebrauchsanweisungen durch ihre Anwender*innen und Entwickler*innen ausführlich dokumentiert und sind in rekonstruierbarer Weise miteinander verbunden.

Die Anwendung von Vermessungstechnologien kann sich in Einzelfällen an Empfehlungen und Anweisungen ausrichten oder sogar verordnet sein, was sie in offensichtlicher Weise zu Regierungstechnologien macht. Der in diesen Texttypen zugrunde gelegte Bedarf, Notstand oder Nutzen sowie die Erscheinungs-

Foucault verwendet die Unterscheidung zwischen Ursprung und Herkunft, um eine antiessentialistische Auseinandersetzung mit Ceschichte zu kennzeichnen. Wohingegen der Ursprung für sich beansprucht, das genuine Inerscheinungtreten einer Idee oder eines Phänomens aufzeigen zu können, markiert die Herkunft das Ergebnis einer selektiven Spurensuche, die alternativen Erzählungen gegenüber aufgeschlossen ist und vor allem praktisch endlos durch die Geschichte weiterverfolgt werden kann (vgl. Foucault (1981 [1969]): 22f.). "[...] Und genau das würde ich Cenealogie nennen, d.h. eine Form der Ceschichte, die von der Konstitution von Wissen, von Diskursen, von Gegenstandsfeldern usw. berichtet, ohne sich auf ein Subjekt beziehen zu müssen, das das Feld der Ereignisse transzendiert und es mit seiner leeren Identität die ganze Geschichte hindurch besetzt« (Foucault 1978: 32). 
form eines Self-Tracking-Tools oder die nahegelegten Nutzungsweisen, ihr Funktionsumfang usw. ist allerdings auch in diesen Fällen bereits diskursiv erarbeitet, wird kontinuierlich verändert oder (re)kombiniert und überwindet daher niemals den Status einer nur vorläufigen Stabilität. Als Kreuzungspunkt verschiedener Diskurse erscheinen alle Technologien des Selbstvermessungsfeldes als Bündelungen von Wiederholungen, Regelhaftigkeiten und Wahrscheinlichkeiten, die nicht durch Subjekte produziert werden, sondern sich als Resultat der Ermöglichung und Verunmöglichung bestimmter Anschlüsse ergeben, an denen unterschiedlichste institutionelle oder nichtinstitutionelle, gegenwärtige und vergangene Diskurspositionen beteiligt sind. Dies macht alle beteiligten Subjekte und Institutionen $\mathrm{zu}$ Produktionsorten von Technologien, verweigert ihnen aber den Status von Produzent*innen - die Urheber*innen von Manualen, Verordnungen und Regelwerken eingeschlossen.

\subsection{Diskursanalyse als induktives Verfahren}

Im Interview »Wer sind Sie Professor Foucault? « (2001[1969]: 776) ${ }^{7}$ gab Foucault an, seine Arbeit ließe sich am besten als »eine Analyse der für unsere Kultur charakteristischen kulturellen Tatsachen definieren. In diesem Sinne handelt es sich gewissermaßen um eine Ethnologie der Kultur, der wir selbst angehören« (ebd.). Demnach setzt die Diskursanalyse, ähnlich wie die verstehende Soziologie nach Alfred Schütz (1972) oder die Ethnografie (vgl. Hirschauer, Amann 1997), eine künstlich eingenommene Naivität gegenüber dem Feld, als ein Forschungsinstrument ein. ${ }^{8}$ Die Diskursanalyse sucht auf diese Weise nach der Methodizität des Feldes selbst und folgt bei seiner Beschreibung den dort vorherrschenden Regeln (Bublitz 2006: 233). Diskurse lassen sich so als geregelte Streuungen von realisierten Aussagen begreifen (vgl. Meier 2008: 24), die sich in Form von Klassifikationen, Taxonomien und Teilungspraktiken erschließen lassen. Diese machen gleichermaßen die subjektivierende Kraft des Diskurses aus.

Der deskriptive Teil der Analyse folgt diesen subjektivierenden Eigenschaften des Diskurses, wohingegen der analytische Teil versucht die Bedingungen zu beleuchten, die zu diesen Klassifikationen führen, die Begründungsmuster und ihre Herkunft zu rekonstruieren, das Fehlen von Aussagen und Positionen, die diskursiven Brüche und institutionellen Zwangs- oder Ausschlusspraktiken sowie seine epistemischen Integrationsstrukturen, Machtpraktiken und Wissensformen herauszuarbeiten (vgl. Bublitz 2003: 58).

$7 \quad$ Zitiert nach Lüders (2007: 92).

8 Zur Offenheit des diskursanalytischen Verfahrens siehe auch Foucault (1981[1969]: 51; 58ff.). 
Vergleichbar auch mit induktiven Forschungsansätzen wie der Grounded Theory (vgl. Glaser, Strauss 2005[1967]) folgt die Diskursanalyse dem Konzept der »aufsteigenden Analyse« (Foucault 2003: 239). Als evolvierende Methode steigert sie ihr konzeptuelles Niveau mit zunehmendem Verlauf, wobei weder thematische Schwerpunkte, Themen oder einzelne Gegenstände die Einheit des Diskurses herstellen, noch Ordnungsstrukturen aus einer vorgängigen Gesellschaftstheorie bezogen werden. Die Klassifikations- und Konstitutionsregeln des Diskurses bilden damit gleichzeitig den Gegenstand und das methodische Werkzeug der Diskursanalyse (vgl. Bublitz 2006: 234f.). Somit erschöpft sich eine an einem Feld oder Phänomen interessierte Diskursanalyse nicht in der Untersuchung einer fest eingrenzbaren oder organisierten Debatte. Vielmehr stellt die Aufdeckung einer diffusen Menge von Diskurselementen und ihrer Relationen die erste analytische Leistung dieser Methode dar. Sie erzeugt damit eine Heuristik für die systematische Beschreibung und Analyse eines Feldes, indem sie den Regeln, Taxonomien, Ordnungen und Systematiken des Feldes selbst folgt. ${ }^{9}$

Vor diesem Hintergrund erscheinen Gouvernementalitätsstudien durch die Fokussierung auf Regierungsweisen und die empirische Einschränkung auf bestimmte Materialtypen als Analysen mit einem bestimmten Analyseziel bzw. einer übergeordneten Rahmenfragestellung.

Nimmt man Diskursanalyse als induktive Methode ernst, lässt lediglich im Verlauf der Forschung, oder im Rückblick auf die Forschung entscheiden, ob es sich um eine Aufdeckung von Regierungsprogrammen handelt, wobei Gouvernementalität das Ergebnis, allerdings nicht der Ausgangspunkt einer Analyse sein kann.

\subsection{Aufsteigende Analyse und aufsteigende Methodenbildung}

Foucault ist in seinen Untersuchungen nicht nur dem Modell der aufsteigenden Analyse, sondern auch dem Prinzip einer aufsteigenden Methodenbildung gefolgt, mit dem er sich schrittweise und »ohne festen Grund emporgearbeitet « hat (DiazBone 1999: 119). Insbesondere die frappierenden Unterschiede zwischen den einzelnen Monografien und ihre nur geringe Bezugnahme aufeinander zeugen von einem »Denken im Prozess«, das an einer »vereinheitlichenden Kanonisierung« kein Interesse entwickelt hat (vgl. Pieper 2006: 269f.). Die Kontinuitätszwänge, die er in historischen und biografischen Narrativen aufdeckt, kontrastiert er mit der 
zeitlichen Bedingtheit des eigenen Denkens: »Jedes Buch verändert das, was ich gedacht habe, als ich das vorhergehende Buch abschloss« (Foucault et al. 1996[1978]: 24). ${ }^{10}$

Wohingegen er in der Ordnung der Dinge (Foucault 1991[1966]) im Zuge der Auseinandersetzung mit den integrativen Ordnungsschemata von Epochen, bzw. der impliziten Regelmäßigkeiten, durch die »in jeder Gesellschaft die Produktion des Diskurses zugleich kontrolliert, selektiert, organisiert und kanalisiert wird « (Foucault 1991[1966]: 10f), kaum eine Reflexion über die Systematik seines Vorgehens lieferte, diente die spätere Archäologie akademischer Wissensbildungsprozesse (Foucault 1981[1969]) vor allem auch als Ordnung der eigenen Methode. Im Zuge dieses Unterfangens machte er also erstmals auch die eigene Regelgeleitetheit und die Selektionsprämissen seiner Beschreibungen zum Gegenstand einer Meta-Reflexion und ermöglichte z.B. durch die Herausstellung verschiedener analytischer Ansatzpunkte wie Gegenstände, Aussagen und ihre jeweiligen Modalitäten zumindest Grundrisse eines reproduzierbaren Vorgehens. Diese diskursiven Formationen (Foucault 1981[1969]: 48ff.) stellen allerdings dennoch eher ein theoretisches Differenzierungssystem, als ein empirisches Forschungsdesign mit klar definierten Regeln dar. »Wer fragt wie Foucault vorgegangen ist, woher er wusste, wonach er suchen muss, wie er die Auswahl seiner Texte durchgeführt hat, wie sich die Hypothesen konstruiert haben, die seine rekonstruktive Analyse angeleitet haben etc., wird die Antwort nicht ohne weiteres finden « (Diaz-Bone 1999: 120).

Die nur schemenhafte Skizzierung des methodischen Verfahrens anstelle einer schematischen Systematik erscheint vor dem Hintergrund der Auseinandersetzung mit der Methodizität von Wissensbildung - also auch der ganz allgemeinen Proklamation einer nur schwer möglichen Trennung zwischen Theorie und Methode - als unumgängliche Konsequenz der zu Grunde liegenden Methodologie und ihres zeitgeschichtlich ausgerichteten Relativismus. So setzt Foucaults Analyse der Ermöglichung und Limitierung von Erkenntnisleistungen nicht erst bei der Methodik von Wissensbildungsprozessen, sondern bereits bei den ihr zugrunde liegenden Begriffen an, die sowohl die Darstellung der Methode als auch die durch sie gewonnenen Erkenntnisse präformieren (vgl. Pieper 2006: 270).

Die Diskursanalyse als mehr oder minder unbestimmtes Amalgam aus Methode, Theorie und epistemologischer Wissenschaftstheorie dekonstruiert nicht lediglich Diskurse im Sinne kontrolliert abgesteckter Forschungsfelder, sondern sie kontextualisiert die Gültigkeit von Wissen anhand vermachteter Ordnungsstrukturen. Dies betrifft nicht nur die universellen Ansprüche wissenschaftlicher Ontologien, sondern grundsätzlich alle Formen der Wissenskonsolidierung, für die auch ihr eigenes Forschungsdesign keine Ausnahme darstellt (vgl. Bublitz et al. 1999: 13f.). 
Bemisst man Foucaults Diskursanalyse an ihren eigenen Prämissen, kann auch sie sich von den Wirkmechanismen der Perspektiven- und Kontextgebundenheit nicht frei machen und jedes Forschungsergebnis kann immer nur den Status einer temporär geltenden Gültigkeit für sich beanspruchen, die in einer kontingenten Beziehung zu alternativen Lesarten steht und deren Bedeutung sich auch im zeitlichen Verlauf kontinuierlich verändert.

Foucaults eigene Abgrenzung (2002[1970-1975]: 166) von der archäologischen Methode zugunsten des geneaologischen Verfahrens, stellt für die zeitliche Begrenztheit methodischer und theoretischer Modelle selbst ein gutes Beispiel dar. Bereits wenige Jahre nach der "Archäologie des Wissens « bezieht sich sein Untersuchungsinteresse den eigenen Auskünften nach bereits nicht mehr schwerpunktmäßig auf die Analyse des Verweisungssystems der diskursiven Formationen, sondern rückt vielmehr nicht-diskursive Elemente wie Institutionen, Formalismen, Praxen und Architekturen ins Zentrum der Betrachtung. Diese Verschiebung erscheint in den meisten Foucault-Rezeptionen als werksimmanenter Bruch, der etwa die genealogische von der archäologischen Schaffensphase trennt. Entsprechend wird mit der Archäologie ein stärker gegen die strukturale Linguistik gerichtetes Einzelwerk gesehen, dass im Verlauf der späten 1970er Jahre durch umfassende Auseinandersetzung mit verschiedenen Machtmechanismen abgelöst wird, die er in der historischen Kontingenz ihrer Ausdrucks- und Legitimitätsformen untersuchte. Diese Lesart der stringenten Trennung beider Werksphasen hat sich inzwischen weitestgehend etabliert, geht dabei aber oft etwas leichtfertig über ihre Zusammenhänge und Parallelen hinweg.

Obgleich eine Fokussierung auf konzeptionelle Ähnlichkeiten unterschiedlicher Monografien eine Navigation innerhalb des ohnehin nicht leicht zu überblickenden Werks einerseits erschwert, wirkt sie andererseits dem impliziten Drängen entgegen, sich mit einer an Foucault anschließenden Analyse für oder gegen bestimmte methodische Prämissen einzelner Werksabschnitte zu entscheiden, die wiederum quer zu den dort verhandelten Phänomenen und Theorien liegen.

Denn so wie man über gouvernementale Programme sagen kann, dass sich ihre gesellschaftliche Relevanz (fernab formalistischer und institutioneller Regeln) über den Diskurs realisiert, lassen sich die diskursiven Formationen wiederum aus der genealogischen Perspektive als historisch kontingent betrachten und in einen machttheoretischen Zusammenhang mit nicht-diskursiven Praktiken, Architekturen oder juristischen Formen bringen. Foucault selbst ist in Bezug auf die eigenen Wechsel zwischen methodischen Fragmenten und Interessensgebieten ein eher pragmatischer Umgang zu unterstellen. In Bezug auf die durch ihn eingeschlage- 
nen Forschungswege, seine Ideen, Modelle, sowie Skizzen und Instrumente sagte er: »Machen Sie damit, was Sie wollen« (Foucault 1999[1975-1976]: 7f.). ${ }^{11}$

Auch wenn jeder spezifische Analyseschwerpunkt notwendiger Weise immer auch eine spezifische Gewichtung methodischer oder theoretischer Fragmente verlangt, müssen deren Eigenheiten beim Wechsel des Erkenntnisinteresses nicht völlig aufgegeben werden. So gibt es im Grunde keinen Anlass, eine Auseinandersetzung mit Machtfragen auf das methodische Repertoire der Foucault'schen Analysen zu beschränken, in denen er Macht selbst als expliziten Analysegegenstand ausgewiesen hat. Ganz im Gegenteil scheinen die an Foucaults Genealogie orientierten Machtanalysen bisweilen hinter der epistemologischen Grundsätzlichkeit der Machtkonzepte zurückzubleiben, die bereits in der Archäologie angelegt ist. Die nicht selten anzutreffende Aussage etwa, dass im Diskurs nicht alles Gehör findet, was gesagt werden kann (vgl. van Dyk und Angemüller 2010), ist bereits als ein institutionalistischer Bias in der Anwendung Foucault'scher Theorie zu werten, der Machtverhältnisse auf beobachtbare Ungleichheiten, Asymmetrien oder Kräfteverhältnisse reduziert. ${ }^{12}$ Dabei ist die Machttheorie Foucaults, unabhängig von beobachtbaren Kräfteverhältnissen auf der Ebene der Sprechorte und Distributionskanäle, bereits viel tiefer mit einer kritischen Epistemologie verwoben, von der aus schon als Machtfrage erscheint was überhaupt gesagt oder gedacht werden kann. Wenn Foucault in der »Archäologie des Wissens« formuliert, dass der manifeste Diskurs immer auch die repressive Tendenz dessen darstellt was nicht gesagt werden kann (Foucault 1981[1969]: 39), zielt dies nicht nur auf die unmittelbare Unterdrückung bestimmter Wissensformen und Selbstverhältnisse (bzw. ihre Förderung - um die produktive Komponente des Foucault'schen Machtbegriffs mit zu benennen), sondern weist auch auf die latenten Bedingungen hin, mit denen Wissen als langsam sich verstetigende Form von Aussage-GegenstandRelationen entsteht und auf dieser Ebene bereits zu einer gleichzeitigen Ermöglichung und Verunmöglichung des überhaupt denkbaren führt. Mit dem Archiv und dem historischen Apriori liefert Foucault zwei verschiedene Konzepte für kollektive Denkschemata, durch die diskursive Aussagen und Aussagebedingungen

Foucault während der Vorlesung »In Verteidigung der Gesellschaft« in Bezug auf die durch ihn eingeschlagenen Forschungswege sowie seine Ideen, Modelle, Skizzen und Instrumente. Zitiert nach Kerchner und Schneider (2006: 9).

12 Foucaul that eine derartige Machtkonzeption selbst vertreten, indem er in seiner Antrittsvorlesung »Ordnung des Diskurses« am Collège de France vordergründig von »Ausschließung«, »Verknappung« und strategischen Regulationsinstanzen im Sinne einer »Diskursiven Polizei« sprach (Foucault und Konersmann 1991[1970]: 25, zitiert nach Pieper 2006: 272). Schon im Rahmen der Folgearbeiten distanzierte er sich allerdings von juridisch-repressiven Machtkonzeptionen und appellierte dafür, Macht und Wissen nicht als per se unterschiedliche Kategorien zu behandeln. 
historisch prädeterminiert werden, ehe es im Machtfeld des Diskurses überhaupt zur Artikulation einer Äußerung kommt.

Foucaults Dekonstruktion jener Ordnung die den Dingen inhärent scheint, liefert so eine Analyse der Denkschemata einer Kultur, die nicht nur das Wissen, sondern auch die Selbstverhältnisse und die sozialen Praxen organisieren (vgl. Bublitz 2003: 45f.). Auch wenn auf das ausgearbeitete Vokabular einer »Archäologie« nicht mehr explizit Anschluss genommen wird, reichen ihre epistemologischen Grundsätze daher in seine Folgeuntersuchungen hinein. 


\section{Herausfinden, ob die Maschine läuft und was sie produziert ${ }^{1}$ Das Instrumentarium der Feinanalyse}

Die kritische Haltung der Diskursanalyse gegenüber Kontinuitäten und genuinen Ursprüngen legt es bereits nahe, den Startpunkt für die Analyse reflexiv, als rein operative Analyseentscheidung, zu begründen. Die Verantwortung für dieses Problem lässt sich ein Stück weit ins Feld selbst verlagern - jedenfalls insofern die Analyse den dort vorherrschenden Selbstauskünften folgt und den dort verhandelten Ursprung zum Startpunkt nimmt. Doch schon bei der Auswahl an relevanten Vorläuferentwicklungen zur Kontrastierung und Dekonstruktion dieser Erzählung bietet sich erneut die Gefahr, den Diskurs, den man analysieren will, anhand externer Prämissen vorzustrukturieren (vgl. Foucault 1981[1969]: 38). Das Projekt der Diskurs-Rekonstruktion kann, wie das der Genealogie, als Geschichte der Gegenwart betrachtet werden, die evidente Ordnungen der Dinge problematisiert, ohne den Dingen dabei selbst auf den Grund gehen zu wollen - »wenn Grund heißt die Dinge oder Sachverhalte einer Letztbegründung, einem ursächlichen Entstehungsund Kausalzusammenhang, einem authentischen Sein, einer kausallogischen Ableitung zuzuführen «(Bublitz 2003: 43). Diskurse sind keine sprachlichen Verarbeitungen von Dingen, die selbst außerhalb des Diskurses liegen. Sie sind entsprechend nicht einer außerdiskursiven Realität untergeordnet, die in soziale Praxis oder Kommunikation übersetzt werden könnte oder deren Wahrheit sich hermeneutisch erschließen ließe. Vielmehr werden Wahrheit und Wirklichkeit historisch diskursiv hervorgebracht (vgl. Bublitz 2003: 56). ${ }^{2}$ Diskurse sind durch andere Diskurse, die mit ihnen Verbindungen eingehen, unentwegt kontextualisiert (Foucault 1981[1969]: 69), dennoch haben Diskurse selektive Relevanzprämissen und Möglichkeitsbedingungen, die sie voneinander unterscheiden. Beide Kriterien wandeln sich zudem im historischen Verlauf. Mit Blick auf die Operationalisierung einer Analyse von Diskursen drängt sich damit unweigerlich die Frage auf, wie dieses 
»Wuchern der Diskurse« (vgl. Foucault 1983[1976]: 119) forschungspragmatisch zergliedert, sortiert, ausgewertet und aufbewahrt werden kann. Wie definiert man Start- und Stopp-Regeln der Untersuchung, wie weit kann, darf oder muss sich der analytische Blick in der Geschichte zurückwenden? Wie umfassend muss die Verzweigung von Teildiskursen und Alteritäten im Binnengefüge des Kerndiskurses untersucht werden?

\subsection{Gegenstände und Formationsregeln - Self-Tracking als Diskursgegenstand}

Ein Forschungsansatz, der darauf basiert, prädiskursive Gewissheiten strategisch in Zweifel zu ziehen, kann die Parameter einer Analyse nicht schlicht mit der Wahl eines (Forschungs-)Gegenstandes bestimmen vielmehr stellt die Beantwortung der Frage was die Gegenstände eines Diskurses sind, selbst einen elementaren Bestandteil der diskursanalytischen Arbeit dar und kann entsprechend nur schrittweise beantwortet werden. Die aufsteigende oder sich ausbreitende Methode folgt nicht dem Prinzip der Definition und Exemplifikation. Der Vorentscheidung dessen, was genau den Kern des Diskurses ausmacht und zu welchem Zeitpunkt er durch welche Ereignisse begonnen hat, ist demnach der mehr oder minder grobe Umriss eines Interesses vorzuziehen von dem aus sich nach und nach der Beantwortung dieser Frage angenähert werden kann. Ein Forschungsinteresse lässt sich leicht als Untersuchung des Self-Tracking-Diskurses oder des Quantified-SelfDiskurses labeln, mit Bedeutung werden diese Begriffe allerdings erst durch das relationale Gefüge ausgestattet, das sie diskursiv konturiert und konstruiert. Die Frage was Self-Tracking ist stellt sich nicht nur der Forschung, sondern auch den Diskursteilnehmer*innen selbst und die Versuche ihrer Beantwortung tragen selbst zur Herausbildung des Diskurses und seiner Binnendifferenzierung bei - z.B. in Subarten des Trackings. So bildet der Diskurs umgekehrt die Gegenstände von denen er handelt (Foucault 1981[1969]: 74).

Ein solcher Ansatz, der von Streuung anstatt von Einheiten ausgeht, tastet (Foucault 1981[1969]: 45) sich langsam anhand empirischer Häufungen und Regelmäßigkeiten voran (Foucault 1981[1969]: 31f.), die er im semiotischen Feld von Aussagen sucht und sie als systematische Zusammenhänge nachweist. Dies sind z.B. Bezüge auf immer wiederkehrende Gegenstände in Form von Aussagen: D.h. »die in ihrer Form verschiedenen, in der Zeit verstreuten Aussagen bilden eine Gesamtheit, wenn sie sich auf ein und dasselbe Objekt beziehen « (Foucault 1981[1969]: 48). Die Analyse verfolgt so serielle Verdichtungen von Aussagen zurück, die als relationales Gefüge im Verbund mit Gegenständen und spezifischen Äußerungsbedingungen den Diskurs bilden. Die Aussage bildet dabei die kleinste kommunikative Einheit der Analyse. D.h. obwohl die Diskursanalyse auch einzelnen Begriffen, 
Satzstrukturen und Schreibweisen eine Bedeutung beimisst, grenzt sie sich von der zeichentheoretischen Fokussierung der strukturalistischen Linguistik ab. Sie vollzieht vielmehr eine analytische Verschiebung auf die konstruktive und konstitutive Funktion von Diskursen, die nicht vordergründig auf eine Beschreibung und Fixierung der sprachlichen Performanz, die Rekonstruktion von Zeichensystemen und des Vokabulars zielt, sondern annimmt, dass eine taxonomische Ordnung der Dinge erst diskursiv hergestellt wird. Entsprechend interessiert sie sich vielmehr für die Differenz dessen, was ausgesagt werden könnte zu dem was letztlich gesagt wird. Nicht nach welchen sprachlichen Regeln Aussagen gebildet werden, sondern warum zu einem gegebenen Zeitpunkt in der Kontingenz möglicher Aussagen eine bestimmte Aussage und keine andere an ihrer Stelle erscheint ist ihre anleitende Frage (Foucault 1981[1969]: 42).

Aussagen sind zwar als Produkte von Äußerungen aufzufassen, allerdings erfolgt ihre sinnhafte Verstetigung erst im Kontext anderer Aussagen auf die sie explizit oder implizit verweisen. Äußerungen werden so nicht kausal aus Identitäten oder dem sozialen Status oder der juridischen Macht abgeleitet die mit bestimmten Sprechorten verbunden ist, sondern sie werden umgekehrt durch die limitierten (Re)kombinationsmöglichkeiten vorgängiger Aussagen (und der Äußerungen anderer) reguliert. Sie stellen somit die Grundbedingung des Wissens dar indem »das Subjekt notwendigerweise angesiedelt und abhängig ist, ohne dass es dort jemals als Inhaber auftreten kann« (Foucault (1981[1969]: 269).

Die Aussagen führt die Diskursanalyse damit nicht auf die psychischen $\mathrm{Zu}$ stände von Sprecher*innen oder Autor*innen zurück, sondern gewichtet etwa die Relationen von Aussagen untereinander - auch dann, wenn diese Beziehungen dem Bewusstsein der Autor*innen selbst möglicherweise entgehen oder es sich um Aussagen von Autor*innen handelt, die zueinander in keiner Beziehung stehen und einander sogar unbekannt sind (Foucault 1981[1969]: 44).

Die durch Gegenstände und Aussagen etablierten Diskurse wirken sich als übergeordnete Möglichkeitsbedingung auf zukünftige Aussagen aus, etablieren Erwartbarkeitszusammenhänge, Wahrscheinlichkeiten, selektive Ausschlussprinzipien und führen so $\mathrm{zu}$ einer sukzessive sich herausbildenden Stabilität des Diskurses, die nicht selten durch Formationsregeln weiter verhärtet oder institutionalisiert wird. Diese immanenten Regeln der diskursiven Formationen wirken sich auf der Ebene ihrer geschriebenen und ungeschriebenen Gesetze auf Diskurse aus, organisieren über geregelte Austauschformate die Zirkulationsbedingungen von Äußerungen oder weisen Sprechorte zu. Zusammengenommen stellen sie die Bedingungen dar, die erfüllt sein müssen, damit eine Aussage im Diskurs anschlussfähig ist. Mit Blick auf den Self-Tracking-Diskurs ist hier etwa an normierte und teils redaktionell kontrollierte Austauschformate $\mathrm{zu}$ denken, die im Verbund mit materiellen und medialen Bedingungen über das Erscheinen von Aussagen (z.B. in moderierten Web-Foren) entscheiden, oder an stark formalisierte, zeitlich 
begrenzte Austauschformate wie sog. Meetups aber auch an nicht-formalisierte Hierarchien in Bezug auf Status und näheren Artikulationsbedingungen zu denen nicht zuletzt die dispositionellen Modalitäten - also die sprachlichen oder medialen Ausdrucksmittel und -fähigkeiten zählen.

Auf der Ebene impliziter Regeln vollzieht sich die Analyse ähnlich wie in Bezug auf die Gegenstände, da sie oft erst im Verlauf der Analyse sichtbar werden und selbst auch ein heuristisches Zugriffsinstrument der Forschung darstellen, durch das der Diskurs beschreibbar wird.

Ein teils konfus wirkendes Sammelsurium an technischen Kuriositäten und unterschiedlichen Biografien, die sich untereinander kaum vergleichen lassen und aktiv auch wenig Bezüge untereinander herstellen, erscheint unter der Annahme der impliziten Regel, der nach im Diskurs z.B. vor allem das Neue, Individuelle und Unkonventionelle prämiert wird, somit als diskursive Produktion von Individualität und der dazugehörigen Technologien.

\subsection{Datentypen und numerische Äußerungsmodalitäten}

Während die Gegenstände ein oder mehrere Zentren bilden, die die (Re)konstruktion des Diskurses erleichtern, stellen die Aussagen die eigentliche Analyseebene dar, auf der sie erscheinen.

Der Diskurs setzt sich zwar aus Aussagen zusammen und bringt diese im Umkehrschluss hervor, die konzeptuelle Offenheit der Diskursanalyse für Äußerungen die nicht aus Sätzen oder Sprechakten gebildet werden, macht sie aber mehr als andere Forschungszugänge sensibel für die immanenten Spezifika unterschiedlicher Diskurse.

So fällt mit Blick auf die Untersuchung der diskursiven Entwicklung von Selbstvermessungstechnologien sehr deutlich auf, dass sich die sprachlich-textlichen Elemente des Diskurses nicht selten um numerische oder diagrammatische Elemente gruppieren ${ }^{3}$, die als spezifische Modalität von Äußerungen, die Gegenstände, Formationsregeln und Begriffe als diskursbildende Elemente ergänzen.

Theodore M. Porter und Bettina Heintz konzeptualisieren numerische Darstellungen als kommunikative Strategien, die persönliches Vertrauen als Voraussetzung für die Überzeugungsfähigkeit einer Aussage minimieren sollen (Porter 1995) oder als symbolisch generalisierte Kommunikationsmedien (Heintz 2007), die bei der Kontingenzbewältigung helfen sollen. Beide Konzepte stellen damit auf die Akzeptanzerhöhung als zentrale Funktion der Zahlen ab. Obgleich sie damit sicher sehr wesentliche Eigenschaften von Zahlen und zahlenbasierten grafischen Darstellungen benennen, legen sie sich allerdings in ihrer Analyse auch stark auf diese fest. Für einen Überblick über die umfangreiche Debatte zu einer Operationalisierung von Diagrammen in den Kultur- und Medienwissenschaften siehe Bauer und Ernst (2010). 
Der Diskurs ist in mehrfacher Hinsicht bestimmt durch derartige numerische Äußerungsmodalitäten, die entweder als gegenseitiger Verweisungszusammenhang mit Text- oder Bildelementen eine Aussage herstellen oder die auf direktem Weg Individuen über die Zahlenaggregate und Durchschnitte interaktiver Bedienoberflächen von Computer-Programmen und Apps vermitteln. ${ }^{4}$

Mit Blick auf die Analyse müssen auch diese nichtsprachlichen Elemente zu den Bedingungen gezählt werden, die beeinflussen in welcher Weise im Diskurs Wissen und Wahrheiten generiert und Subjektpositionen geformt, zugewiesen oder eingenommen werden. Damit ergeben sich eine ganze Reihe an Materialtypen, die für die Analyse relevant sind: Dies sind zum einen verschiedene Textgattungen wie Blog- und Foreneinträge, redaktionell überarbeitete Artikel, Vorträge und Gruppendiskussionen, sowie Protokolle in Textform oder Videoaufzeichnungen, Werbeund Selbstdarstellungstexte (z.B. der Anbieter von Self-Tracking-Systemen aber auch von populären Self-Tracker*innen), sowie die Taxonomien, Klassifikationssysteme, Visualisierungen, interaktiven und dynamischen Elemente der TrackingTechnologien selbst.

\subsection{Korpusbildung - Die selbstregistrative Praxis des Self-Trackings}

Auch im Zuge der Korpusbildung geraten sofort spezifische Eigenheiten des Selbstvermessungsdiskurses in den Blick, die mit der Medialität des Diskurses im Zusammenhang stehen. Dies betrifft z.B. hypertextuelle oder anderweitige medientechnologische Besonderheiten der Diskursorganisation - auch wenn diese Spezifik kein exklusives Merkmal des Self-Tracking-Diskurses darstellt, sondern angesichts der zunehmenden Medialisierung und gleichzeitigen globalen, kommunikativen Vernetzung inzwischen eher den Normalfall darstellt. Fernab aller Affinitäten der Diskursteilnehmer*innen an der systematischen und möglichst umfassenden Verdatung, Speicherung und Auswertung verschiedenster Körper- und Alltagsbereiche, stellt die passive Registration von Kommunikationsfragmenten und ihrer Meta-Daten (wie z.B. die Protokollierung des genauen Erscheinungszeitpunkts von Beiträgen) einen interessanten Nebenaspekt von online-basierter Kommunikation im Allgemeinen dar, der neue Möglichkeiten für die Rekonstruktion sinnhafter Ordnungsstrukturen und Entstehungskontexte von Diskursen eröffnet. Diese Möglichkeiten sind dabei allerdings nicht für die Diskursforschung reserviert, sondern nachweisbar an der Organisation des Diskursgeschehens selbst beteiligt, da sie für alle Diskursteilnehmer*innen offen liegen. So bilden die textbasierten und zeitversetzten Kommunikationsmodi (durch Foren und Weblogs) im Verbund mit der digitalen Infrastruktur des Webs eine selbstregistrative Diskurspraxis aus, die 
sukzessive ein Archiv von Aussagen im Zuge ihrer Äußerung erstellt und durch Hyperlinks $^{5}$ nicht nur über Gegenstandsbezüge oder rekonstruierbare RegelmäBigkeiten und Aussage-Wiederholungen, sondern auch auf technischem Weg eine assoziative Binnendifferenzierung herstellt.

Die technischen Aspekte der Hypertextualität, die Visualität aber auch die Interaktivität von Internetmedien setzen damit die Texte, Bilder und Videos zu multimedialen und multimodalen Mashups unterschiedlicher semiotischer Zeichensysteme zusammen, die im Fall des Self-Tracking-Diskurses zusätzlich durch die bedeutungsgenerierende Funktion numerischer Zeichen und die spezifischen Modalitäten ergänzt werden, in denen diese den Status von Äußerungen und Aussagen annehmen.

Für den Forschungszugang bedeutet dies, dass sich die Korpusbildung einerseits entlang dieser interaktiven Strukturen vollziehen kann, dabei allerdings auch die Eigendynamiken dieser Verweisungsstruktur berücksichtigen muss. ${ }^{6}$

Die aufsteigende Analyse eines Diskurses und seiner selbstregistrativen Online-Praxis verzichtet daher auf die Vorab-Zusammenstellung einer Materialbasis - der Annahme, durch eine Stichprobe eine unverfälschte oder repräsentative Miniatur eines Diskursoriginals herstellen und unter Laborbedingungen untersuchen zu können, folgt sie ohnehin nicht. Denn Diskurse sind gleichzeitig operative Bedingungen und Operationalisierungen sozialer Wirklichkeit, die je nach Perspektive als eine andere erscheint. Zudem handelt es sich gerade bei gegenwärtigen Onlinediskursen immer nur um fluide und instabile diskursive Konfigurationen, die sich im zeitlichen Verlauf (d.h. schon im Zuge ihrer Analyse) rapide verändern.

Die Auswahl des Analysematerials erfolgt daher schrittweise, beginnt auf der Ebene der Gegenstände und folgt den diskursinternen Differenzierungskategorien, Häufungen und Verweisungsstrukturen, um so nach und nach ein immer deutlicher werdendes Bild des Diskurses entstehen zu lassen, das auf diese Weise bis zum Ende der Analyse eine Verbindung zum Diskursgeschehen beibehält und sensibel für aktuelle Ereignisse bleibt.

Dieses Vorhaben erleichtern konventionelle Suchmaschinen aber auch Wayback-Maschinen, die den Zugang $\mathrm{zu}$ bereits gelöschten Websites oder

In der Methodendiskussion der »Webnografie«bzw. »Virtual Ethnography« ist gut dokumentiert, wie sich die interaktiven Verweisstrukturen des Webs, wie z.B. Feeds und Tags, für eine Analyse einsetzen lassen. Siehe vor allem Hine (2000).

6 Eine solche Eigendynamik kann z.B. die medientechnische Bildung rekursiver Verweisungsstrukturen sein, die zu einer Schließung gegenüber der übrigen Diskursteile tendieren. Das Verfolgen und Aufdecken derartiger Verweisungsgeflechte kann analytisch von großem Wert sein, sie erfordern allerdings auch von Zeit zu Zeit neue Zugänge zum Diskurs zu schaffen, um der Varianz der Aussagen und Gegenstände gerecht zu werden und nicht zu stark auf Einzelaspekte zu fokussieren. 
Blogeinträgen erlauben - also zu Material, dass von den Hosts der materiellen Archivstruktur selbst wieder entfernt wurde. ${ }^{7}$ Neben den Gegenständen des Diskurses, stellen auch spezifische Sprechorte wie institutionalisierte Funktionsträger*innen Analysequellen dar, denen im Diskurs sehr präsent bestimmte Attribute wie die Erfindung bestimmter Technologien, Namensprägungen oder die Gründung lokaler Communities zugeschrieben werden. Aber auch die Vermessungstechnologien selbst, bzw. die mit ihnen verbundenen Selbstbeschreibungen gehen in die Auswertung ein.

Von diesen Startkoordinaten bewegt sich die aufsteigende Analyse schließlich immer weiter in die Peripherie der Kommentare, Produkttests, technologischen Aneignungen und Zweckentfremdungen. Sie kann mit der Analyse eines populären Zeitungsinterviews starten und sich über die Kommentare, die andere SelfTracker*innen hinterlassen haben, zu ihren Weblogs, den öffentlichen Protokollen ihrer Vorträge oder ihrer dokumentierten Selbstexperimente und Konstruktionsanweisungen zugehöriger Vermessungstechnologien ausweiten. Gleichzeitig kann sie auch den selbstreferentiellen Verweisungsstrukturen des institutionalisierten Kerndiskurses, also einer intendierten Vorauswahl und Leserichtung folgen, die etwa durch Community-Organisator*innen nahegelegt wird. Hierbei helfen die mit Blog-Einträgen zusammenhängenden Tags, die Suchfunktionen der Quantified-Self-Website sowie Newsletter, und Social-Media-Accounts. Einen weiteren Ansatzpunkt bilden die Community-Events selbst. Internationale Konferen-

$7 \quad$ Natürlich beeinflussen die technischen Infrastrukturen des Internet und World Wide Web selbst das Diskursgeschehen durch eine enorme Selektivität in Bezug auf die Sichtbarmachung von Dokumenten. Insbesondere die Algorithmen von Suchmaschinen geraten immer wieder in den Fokus entsprechender Kritiken und Forschungsarbeiten. Auch die Zusammenstellung des Analysematerials ist, insofern sie entsprechende Anwendungen nutzt, damit unweigerlich immer bereits durch opake Kriterien der Aufmerksamkeitsverteilungen im Internet unterminiert. Zudem vollziehen sich die Selektionsmechanismen von Suchmaschinen zunehmend individualisiert, was die Antizipation der Reichweite und Popularität einzelner Websites und Blogs erschwert. Eine beispielhafte Analyse, die mit einem an Foucault anschließendem Instrumentarium operiert, stellt etwa eine Dispositivanalyse der GoogleSuchmaschine durch Theo Röhle dar, anhand derer die Musterbildungen in Suchmaschinenergebnissen als rekursive Konstellationen zwischen menschlichen und nichtmenschlichen Elementen untersucht wurden (2010: 16). Obgleich das forschungspragmatische Problem der individualisierten Selektivität der eigenen Suchmaschinennutzung durch das Alternieren der Suchmaschinenanbieter und die Verwendung von Proxy-Servern zumindest ansatzweise kontrolliert werden kann, lässt sich der Effekt, den derartige Mechanismen auf die Diskursbildung haben, anhand der hier zur Verfügung stehenden Mittel nur schwer ermessen. Einem solchen technischen Bias kann entsprechend nur durch die Verbreiterung der Analysezugänge (etwa die Materialerhebung im Rahmen von Offline-Veranstaltungen) sowie durch die explizite Berücksichtigung von Querverweisen innerhalb der untersuchten Dokumente entgegen gearbeitet werden. 
zen, lokale Treffen aber auch spezifische Fachmessen zeugen etwa von der Verflechtung des Self-Tracking mit Diskursen des Gesundheitswesens oder der Personalentwicklung. Diese kontinuierliche Folge aus Analyse, Erhebung erneuter Analyse und weiterer Materialsuche changiert so zwischen Kerndiskurs, seiner (z.B. journalistischen) Peripherie und den vernetzten Interdiskursen.

Die vorliegende Arbeit hat auf diese Weise ein ambivalentes Verhältnis in Bezug auf spezifische Teilaspekte und die allgemeine Bedeutung des Gegenstandes entfaltet. Wohingegen die Materialsuche weitestgehend allgemein begann und sich nach und nach selektiv spezifischen Aussagefolgen und diskursiven Unterthemen zugewandt hat - in dem sie z.B. den diskursiv ausgehandelten Zusammenhängen zwischen Stress-Tracking und Produktivitätsmessungen mehr Gewichtung beigemessen hat als dem Zusammenhang zwischen ernährungsbezogenem Tracking und der Vermessung von sportlicher Leistung - untersucht sie diese spezifischen Gegenstände zunächst auf dem Niveau individueller Selbstexperimente und verfolgt ihre allgemeine Popularisierung, Verbreitung und Appropriation durch staatliche oder wirtschaftliche Agenturen.

Die Versuchung, die verstreuten Elemente zu einer kontinuierlichen Fortschrittsoder Verfallsgeschichte zusammenzufügen oder den im Diskurs selbst ausgewiesenen Ursprungsnarrativen $\mathrm{zu}$ folgen, ist dabei allgegenwärtig, wird jedoch immer wieder durch die Komplexität und Verstrickung mit diskursiven $\mathrm{Ne}$ benschauplätzen irritiert, die mit den Gegenständen des Kerndiskurses eigene Sinnzusammenhänge bilden und daher alternative Lesarten nahelegen.

Auf diese Weise ist eine Materialbasis von ca. 300 unterschiedlichen (Internet-)Dokumenten entstanden, die in die Analyse eingegangen sind, d.h. die systematisch in Textform und anhand von Screenshots gespeichert, $\mathrm{zu}$ thematischen Schwerpunkten gruppiert und verschlagwortet wurden. Die in ihnen enthaltenen Verweise auf andere Dokumente wurden zudem hervorgehoben und kleinschrittig bearbeitet. $\mathrm{Zu}$ der Materialbasis zählen allerdings auch Dokumente in Papierform, wie z.B. Informationsmaterial von Messeständen, Prospekte von Krankenkassen oder Printversionen von Zeitschriften und Magazinen. Zudem sind auch einige Audioaufzeichnungen in die Analyse eingegangenen, die während verschiedener Meetups, Konferenzen oder Vorträge und Panels auf Messen entstanden sind.

\subsection{Herkunft und Ursprung - Diskursanalyse, Genealogie und Narrativität}

Die Diskursanalyse muss aus der Perspektive ihrer archäologischen Rekonstruktion notwendiger Weise unvollständig bleiben, denn die Archäologie kann die Gesamtheit der Bedingungen ihrer Gegenstände niemals ganz erfassen (Bublitz 2003: 80). Das Erfordernis, die Netzwerkartigkeit und Zirkularität diskursiver Forma- 
tionen im Rahmen der Ergebnisverschriftlichung auf eine weitestgehend lineare Textform zu reduzieren, führt zudem dazu, dass sich nicht nur durch die Auswahl der Analyseelemente, sondern auch die Festlegung ihrer Reihenfolge in der Darstellung ein übergeordnetes Narrativ entwickelt, dessen Startpunkt letztlich eine Selektionsentscheidung darstellt. Nichtsdestoweniger macht es einen entscheidenden Unterschied, ob sich diese Narration der Herstellung einer hermetischen Kohären $z^{8}$ verpflichtet, die gerade darauf basiert Inkonsistenzen argumentativ zu überdecken oder ob sie Raum für die detaillierte Beleuchtung der Widersprüchlichkeit und Deutungskämpfe diskursiver Prozesse lässt und diese ihrerseits in den Fokus der Analyse rückt.

Diese Differenz lässt sich dabei nicht einfach auf die binäre Unterscheidung zwischen einem Kontinuitäts- und Diskontinuitätsnarrativ übertragen. Vielmehr geht die Suche nach den Ursprüngen oder »ersten Vorläufern « (Foucault 1981[1969]: 11) eines Phänomens nicht selten Hand in Hand mit einer Einteilung der Geschichte in Epochen und Entwicklungsphasen im Namen historischer Zäsuren. ${ }^{9}$ Die Differenz stellt sich vielmehr dadurch ein, dass operative Ordnungsmechanismen als diese reflektiert und nicht als überzeitliche Ontologien behandelt werden. ${ }^{10} \mathrm{Ei}$ -

8 Hierbei ist neben den »linearen Abfolgen«, denen die Geschichtsschreibung über Jahrhunderte folgte, auch an die »langen Perioden« als artifizielle Unterteilungen zu denken, auf die sich die Aufmerksamkeit jüngerer historischer Studien richtet und die nach Foucault in ganz ähnlicher Weise bemüht sind »unter den politischen Peripetien und ihren Episoden die festen und nur schwer zu störenden Cleichgewichte, die irreversiblen Prozesse, die konstanten Regulierungen, die Phänomene mit der Tendenz, nach jahrhundertelanger Dauer ihren Höhepunkt zu erreichen und umzuschlagen, die Akkumulationsbewegungen und langsamen Sättigungen, die großen, unbeweglichen und stummen Sockel, die die Verschachtelung der traditionellen Berichte mit einer dicken Schicht von Ereignissen bedeckt hatte, zum Vorschein zu bringen« (Foucault 1981[1969]: 9).

9 Wie Foucault beschreibt, folgt das Vorhaben jeder Form einer »allgemeinen Ceschichte « und ihrer Unterteilung in Perioden und Epochen verschiedenen Hypothesen: »man nimmt an, dass unter allen Ereignissen eines räumlich-zeitlich wohl abgesteckten Bereichs, zwischen allen Phänomenen, deren Spur man wiedergefunden hat, sich ein System homogener Beziehungen feststellen lassen muss: ein Netz der Kausalität, das die Ableitung eines jeden von ihnen gestattet, Analogiebeziehungen, die zeigen, wie sie einander symbolisieren oder wie sie alle ein und denselben zentralen Kern ausdrücken; man nimmt andererseits an, dass ein und dieselbe Form von Geschichtlichkeit die ökonomischen Strukturen, die sozialen Stabilitäten, die Unbeweglichkeiten der Mentalitäten, die technischen Gewohnheiten, das politische Verhalten hinwegschwemmt und alle dem selben Transformationstyp unterwirft; man nimmt schließlich an, dass die Geschichte selbst in große Einheiten gegliedert werden kannStadien oder Phasen, die in sich selbst ihr Kohäsionsprinzip enthalten « (Foucault 1981 [1969]: 19).

10 Diese Differenz lässt sich zudem auch nicht als stringenter Kontrast zwischen empirischen Äußerungen des Phänomenbereichs auf der einen und wissenschaftlichen MetaÄußerungen auf der anderen Seite abbilden, da die Festsetzung von Ursprüngen oder Rah- 
ner historischen Theoretisierung, die komplexe Neuerungen der Gegenwart auf ein genuines Genie - und damit auf eine »den Individuen eigene Entscheidung" zurückführt (Foucault 1981[1969]: 33f.) oder sie über Jahrhunderte hinweg als chronologische Vernunft-Kontinuität beschreibt (vgl. Foucault 1981[1969]: 17), ${ }^{11}$ stellt die Genealogie gerade die Problematisierung einer evidenten Ordnung der Dinge gegenüber (Foucault 1991[1966]). Also die kritische Betrachtung augenscheinlicher Zusammenhänge auf der Ebene der Wörter und der Sachen (Foucault 1981 [1969]: 74), ${ }^{12}$ $\mathrm{zu}$ denen vor allem die ursächlichen Entstehungs- und Kausalzusammenhänge zählen, der sich eine solche Ordnung selbst bedient, um eine Aussage über die Geschichte als authentisch zu begründen (vgl. Bublitz 2003: 43). Diskursanalytisch zugespitzt bedeutet dies, dass sie die Geschichte eines Begriffs nicht als »die seiner fortschreitenden Verfeinerung, seiner ständig wachsenden Rationalität, seines Abstraktionsanstiegs, sondern [als] die seiner verschiedenen Konstitutions- und Gültigkeitsfelder, die seiner aufeinander folgenden Gebrauchsregeln, der vielfältigen theoretischen Milieus« betrachtet, »in denen sich seine Herausarbeitung vollzogen und vollendet hat « (Foucault 1981[1969]: 11) - ihr Interesse widmet sich vielmehr dem Infragestellen der linearen Ideengeschichte selbst (Foucault 1981[1969]: 27).

mungen anhand historischer Großnarrative Instrumente sind, die nicht auf einzelne Diskurse festgelegt sind. Sie finden sich sowohl in den Cründungsmythen einer Community wie Quantified Self, als auch in wissenschaftlichen Versuchen das Phänomen der Selbstquantifizierung in eine allgemeine Kulturentwicklungsgeschichte einzugliedern. Zudem wird das Narrativ der Kontinuität auch nicht selten durch Verweise auf die historischen Analysen Foucaults ausgeführt - ganz gleich ob es sich dabei um die Analyse von Diskursen oder von Praxen, Technologien und Institutionen handelt. Dies sicher auch deshalb, da Konzepte wie das der Technologie des Selbst von der »mikroskopischen « Detailanalyse und thematischen Kontextualisierung (Foucault 1981 [1969]: 11) leben, in die sie eingebettet sind und ohne ihre konkreten Bezüge zu vergleichsweise schemenhaften Allgemeinplätzen verblassen, die sich dann genauso gut auch durch Analysen besetzen lassen, die der historischen Parallelisierung und der kontinuierlichen Entwicklungsgeschichte den Vorrang vor einer Beleuchtung der Kontroversen und Diskrepanzen historischer Cemengelagen geben.

11 Bei den Versuchen geschichtlicher Kontinuitätskonstruktion, als auch denen der fiktiven Konstruktionen eines ursprünglichen, aufklärerischen Subjekts, handelt es sich letztlich um »die beiden Cesichter ein und desselben Denksystems« (Foucault 1981 [1969]: 23).

12 »Die Wörter und die Sachen« ist die wörtliche Übersetzung des französischen Originaltitels der in Deutschland unter dem Titel »Die Ordnung der Dinge« (1991 [1966]) erschienenen Analyse der Humanwissenschaften (Foucault 1981 [1969]: 74). 


\subsection{Populäre Ursprungsnarrative des Self-Trackings}

Ein populäres Ursprungsnarrativ der intrinsischen und selbstbezogenen Vermessung, das vor allem in der journalistischen ${ }^{13}$ und wissenschaftlichen ${ }^{14}$ Entourage der Quantified-Self-Community als Beginn des Self-Tracking behandelt wird, bezieht sich auf eine spezielle Protokolltechnik Benjamin Franklins, die in seiner Autobiografie dokumentiert ist (vgl. Franklin 1916[1793]: 69). So protokollierte Franklin tabellarisch jeden seiner Verstöße gegen selbst aufgestellte und zu Tugenden generalisierte Verhaltensnormen.

»These names of virtues, with their precepts, were: 1 . Temperance. Eat not to dullness; drink not to elevation, [...] 2. Silence. Speak not but what may benefit others or yourself; avoid trifling conversation. [...] 3. Order. Let all your things have their places; let each part of your business have its time. [...] 5. Frugality. Make no expense but to do good to others or yourself; i.e., waste nothing. [...] 6. Industry. Lose no time; be always employ'd in something useful; cut off all unnecessary actions. [...] 10. Cleanliness. Tolerate no uncleanliness in body, clothes, or habitation. ${ }^{15}$

Die Autorin Catharina Koller schreibt dazu: »Das Prinzip stammt von Benjamin Franklin: [...] regelmäßig führte der die Liste über dreizehn Tugenden, ihre Einhaltung und Verfehlung. Ganz ähnlich sammeln heute Selbstquantifizierer Daten über sich. Neu ist die automatische Erhebung. Wer mit einem dünnen Sensorenstirnband seinen Schlaf überwacht, muss beim Aufwachen nicht mehr die Uhrzeit notieren. Das macht die Technik. «Quelle: www.zeit.de/2 012/07/WOS-Quantified-Self (zuletzt aufgerufen am 03.02.2015).

So schreiben etwa Dawn Nafus (Senior Research Scientist in den Intel Labs) und Dr. Gina Neff (Associate Professor of Communication and Senior Date Science Fellow am eScience Institute an der Universität Washington): »People keep track. In the eighteenth century, Benjamin Franklin kept charts of time spent and virtues lived up to. Today, people use technology to selftrack: hours slept, steps taken, calories consumed, medications administered.«Quelle: ww w.datasociety.net/output/the-quantified-self/ (zuletzt aufgerufen am 06.05.2016). Und auch Simon Schaupp folgt im streng wissenschaftlichen Sinne dieser Kontinuitätsgeschichte in einer aktuellen Publikation zum Thema Self-Tracking: »]ahrhunderte später entwickelte der US-Amerikanische Staatsgründer Benjamin Franklin diese Technik unter explizitem Bezug auf die antiken Philosophen, insbesondere Pythagoras, weiter« (2016: 153). Darüber hinaus stellt auch in Bezug auf verschiedene Selbsthilfepraktiken, unter denen das Self-Tracking gemeinhin subsummiert wird, Benjamin Franklin ein oft bemühtes Ursprungsnarrativ dar. So z.B. bei Antje Czudaj's Studie »The Creative Class between Self-Help and Individualism« (2016: 10), die sich dort auf die Autor*innen Steven Starker (1989: 15) und Micki McCee (2005: 27) bezieht. Auch Stefan Meißner folgt dieser Erzählung (2016: 217) und Richard Barbrook resümiert in seiner Kritik der Kalifornischen Ideologie: »The path of technological progress didn't always lead to »ecotopia « - it could instead lead back to the America of the Founding Fathers«(Barbrook und Cameron 1995). Benjamin Franklin (1916[1793]: 69). 
Insbesondere in resümierenden Überblicksbeschreibungen werden die numerischen Selbstbezüge Franklins dabei häufig als rudimentäre Vorform des Self-Tracking beschrieben, dessen Potentiale sich, angestoßen durch den technologischen Wandel, erst unter Gegenwartsbedingungen massenhaft $\mathrm{zu}$ entfalten und verbreiten beginnen.

»This is not new. Benjamin Franklin famously tracked 13 personal virtues in a daily journal to push himself toward moral perfection. He shared this insight in his autobiography: »I was surprised to find myself so much fuller of faults than I had imagined, but I had the satisfaction of seeing them diminish «. The only difference today is the technology. ${ }^{16}$

Tatsächlich ist mit einem Projekt des Designers Joe Dizney aus dem Jahr 2009 bereits ein früher Versuch dokumentiert, die historische Figur Benjamin Franklin im Sinne der Quantified-Self-Community als "fascinating 18th century self-quantifier « ${ }^{17}$ $\mathrm{zu}$ besetzen und die Community, über die ideelle Verflechtung mit der Biografie dieser symbolischen Pionierfigur, historisch in der US-Amerikanischen Kultur zu lokalisieren. ${ }^{18}$ Anscheinend inspiriert durch eine populäre Graphic Novel der Journalistin und Illustratorin Maria Kalman aus der New York Times ${ }^{19}$, erprobte Dizney die Protokollierung der 13 Tugenden Franklins 90 Tage lang an sich selbst und stellte das Selbstquantifizierungsprojekt über einen Show\&Tell-Vortrag stellvertretend zur Diskussion innerhalb der New Yorker Quantified-Self-Community.

Obgleich über die Verwendung dieser rudimentären Form numerischer Protokollierung und auch mit Blick auf die jeweiligen Bereiche der Protokollierung eine gewisse Parallelität zwischen aktuellen Selbstvermessungsprojekten und der sorgvollen Selbstzuwendung Franklins augenscheinlich ist, stellen sich jedoch bei ei-

16 Aus: »The Beginners Guide to Quantified Self.« Quelle: http://technori.com/2013/04/4281-th e-beginners-guide-to-quantified-self-plus-a-list-of-the-best-personal-data-tools-out-there/ (zuletzt aufgerufen am 17.06.2016). Eine vergleichbare Kontinuitätsthese wird auch unter den kritischen Betrachter*innen des Phänomens vertreten. So schreibt Corinna Schmechel: »In diesem Sinne stellen Techniken wie Self-Tracking lediglich die eine, dem Stand der Technik und politischen Dispositive (als Stichworte seien hier Biopolitik und Couvernementalität eingeworfen) entsprechende, Form der Technologien des um sich sorgenden Selbst dar « (2016: 180).

17 Quelle: http://quantifiedself.com/2009/09/nyc-qs-showtell-2-graffito-c/ (zuletzt aufgerufen am 06.05.2016).

18 Damit schließen sie an viele populäre Erzählungen an, die unternehmerisches Handeln und Erfindungsreichtum per se mit dem Geist der »Cründerväter « gleichsetzen. In einer emphatischen Rede aus dem Jahr 1985 forderte etwa der damalige US-Präsident Reagan die Amerikaner*innen auf, die Zukunft in ähnlicher Weise zu erfinden, wie es ihre Vorfahren zwischen Bürgerkrieg und Großer Depression getan haben (vgl. Bröckling 2007: 54).

19 Quelle: http://kalman.blogs.nytimes.com/2009/07/30/can-do/ (zuletzt aufgerufen am 06. 05.2016). 
ner subjektgeschichtlichen Kontextualisierung Zweifel an der Belastbarkeit dieser numerischen Selbstbeobachtung als archaische Vorform aktueller Selbstvermessungsmaxime ein.

Durchaus lassen sich sowohl die numerischen Selbstzuwendungen Benjamin Franklins als auch der Quantified-Self-Mitglieder als "gewusste und gewollte Praktiken« beschreiben, »mit denen die Menschen nicht nur die Regeln ihres Verhaltens festlegen, sondern sich selber zu transformieren, sich in ihrem besonderen Sein zu modifizieren suchen « (Foucault 1986: 18). Beide weisen damit auch Parallelen zum Konzept der Technologien des Selbst auf. ${ }^{20}$ Aus derartigen Ähnlichkeiten auf kategorialer Ebene lässt sich allerdings kaum eine Aussage von subjektanalytischer Relevanz treffen. Sowohl die vergleichsweise einfach herstellbaren Bezüge zu dem allgemeinen Konzept der Selbstsorge, als auch ihre lose Kombination mit numerischen Verfahren, reichen allein nicht aus, um die Vermessungen Franklins als

Das Konzept der Technologien des Selbst nimmt auch in vielen weiteren mehr oder minder an Foucault orientierten Untersuchungen des Selbstvermessungsphänomens einen wichtigen Stellenwert ein. Hier ist etwa an die zahlreichen Versuche zu denken, antike Selbstpraktiken als Vorformen des Self-Tracking zu identifizieren um so z.B. eine Analyse des Ernährungsoder Diät-Trackings über eine historisch argumentierende Steigerungshypothese zu einer allgemeinen Geschichte von Selbstzuwendungsformen zu erweitern. So z.B. bei Zillien et al. (2016), die digitales Diät-Tracking aus der Perspektive einer »gezielten Verwissenschaftlichung der Ernährung « betrachten und argumentieren, dass etwa bereits entsprechende Lehren des Hippokrates »von Beginn an« mit Quantifizierung verbunden waren (Zillien et al. [2016: 123). Dabei beziehen sie sich u.a. auf Foucaults Untersuchungen zu antiken Existenzkünsten, denen nach etwa Körper und Seele »Dinge« darstellen »die gemessen sein müssen« (Foucault 1984: 131). Auf die gleiche Textstelle aus dem zweiten Band der Reihe »Sexualität und Wahrheit« beziehen sich auch Corinna Schmechels Verknüpfungen digitaler Selbstvermessungsverfahren mit der antiken Diätetik (2016: 179f.). Obgleich ein Zusammenhang zwischen antiker Diätetik und digitalen Technologien zur Diätüberwachung sicher unverkennbar ist, stellt sich die Frage, ob die Begriffe, Praxen und Technologien die sie ausmachen dadurch automatisch in einer homogenen und linearen Entwicklungsbeziehung zueinander stehen müssen, die zudem allesamt dem gleichen Transformationstyp (wie z.B. der Verwissenschaftlichung) unterliegen. „Dies umso mehr, wenn es sich darum handelt, Mengen von Aussagen zu analysieren, die in der Epoche ihrer Formulierung einer völlig anderen Distribution, Aufteilung und Charakterisierung unterlagen « (Foucault 1981[1969]: 35). Zudem existieren eine ganze Reihe ähnlich gestrickter Forschungsarbeiten, die anstelle der Diätetik allerdings die ebenfalls durch Foucault beschriebenen Techniken des Selbstschreibens in der Form von Hypomnema (vgl. Foucault 2005[1980-1988]: 507f.), die schriftliche Korrespondenz als eine produktive und dynamische Form »sich dem anderen und sich selbst zu zeigen« (Foucault 2005[1980-1988]: 515) oder das Tagebuchschreiben zum Ursprung reflexiver Protokollpraktiken erklären, das mit Anbruch der Moderne als Erbe der katholischen Ohrenbeichte schrittweise zu einer sekularisierten Technologie der Selbstherstellung avanciert. Vor dem Hintergrund dieser Technologien muss die subjektgeschichtliche Bedeutung von Formen der numerischen Selbsterforschung über lange historische Zeiträume hinweg im Grunde als marginal bezeichnet werden. 
eine Praktik zu beschreiben, die unter den Bedingungen des 21. Jahrhunderts ihre Entsprechung findet.

Zwar lassen sich die Überwachung von Kalorienaufnahme, Alkoholkonsum, der körperlichen Hygiene sowie der Zeiteffizienz verschiedener Tätigkeiten leicht unter den Motiven gegenwärtiger Selbstvermessungspraktiken ausmachen, aus subjektanalytischer Perspektive kann von einer Kontinuität allerdings erst dann gesprochen werden, wenn über die Objekte und Technologien der Vermessung hinaus auch die übergeordneten Subjektivationsziele - also das angestrebte Selbstbild eine Analogie in den Self-Tracking-Praktiken der Gegenwart findet. Aus dieser Perspektive ist jeder Thematisierung historischer Vorformen von Selbstvermessung als ideelle Fortschrittsgeschichte vielmehr die Frage danach entgegen zu setzen, welche historisch spezifischen Wissensformen die Fragen nach dem Selbst und schließlich seiner praktischen Formung jeweils anleiten (vgl. Rose 1996: 23; Bröckling 2012: 133f.).

Es gilt inzwischen beinahe als voraussetzungsfähig, dass die Quantified-SelfMitglieder zu diesem Zweck auf unkonventionellem Weg die Generierung eines Wissens über sich selbst vorantreiben, das sich in einem zweiten Schritt für die intrinsische Aktivierung ungenutzter Potentiale nutzbar machen lässt. Das SelfTracking befragt die Routinen des Alltags auf ihren ökonomischen Gehalt und sucht nach Verbesserungsmöglichkeiten jenseits der plattgetretenen Pfade täglicher Gewohnheiten. Die sich hierin begründenden Selbstziele koinzidieren dabei in starkem Maße mit der Art und Weise »in der Personen im politischen Vokabular der fortgeschrittensten liberalen Demokratien vorkommen« - nicht mehr ausschließlich als Subjekte mit Pflichten, sondern als Träger*innen von Rechten und Freiheiten, die zudem Autonomie und Wahlfreiheit selbst als Wert, Ideal und Ziel politischer Praxis vertreten (Rose 2000: 8). Es ist insofern ein Phänomen der Moderne.

Tatsächlich bringt der kulturgeschichtliche Wandel des späten 18ten Jahrhunderts, in dem die Selbstvermessungspraktiken Franklins situiert sind, eine unübersichtliche Gemengelage aus unterschiedlichen Subjektivierungstechniken hervor, die sich über religiöse Souveränität hinaus, erstmals auch an allgemeineren Prinzipien wie politisch-ökonomischer Rationalität ausrichten (Sarasin 2001: 22). Wobei nicht zuletzt Franklins dreizehnte Tugend »Humility. Imitate Jesus and Socrates ${ }^{21}$ allerdings ein Ausdruck davon sein mag, dass der primäre Subjektivierungsmodus $\mathrm{zu}$ dieser Zeit noch frei flottierend zwischen einer Orientierung an religiöser Pastoralmacht und den vermehrt aufkommenden selbstregulativen Steuerungstechniken des demokratischen Liberalismus changiert (vgl. Foucault 2004[1978-1979]: 442). 
Obgleich die Grundzüge einer politischen Rationalität der »avisierten Kongruenz zwischen einem verantwortlich-moralischen und einem rational kalkulierenden Subjekt « (Lemke 2000: 39), das auch die gegenwärtigen Self-Tracking-Praxen anzuleiten scheint, damit zumindest schemenhaft bereits in dieser Zeit angelegt sind, tendiert Franklins rudimentäre Zählung von Alltagssünden dennoch vergleichsweise einseitig zu der disziplinierten Einhaltung traditioneller, religiösmoralischer Kodizes, in einer alternierenden Bewegung zwischen Selbstformung und protonormalistischer Selbstvergewisserung - an deren Ende die fromme Demut steht. ${ }^{22}$

»My intention being to acquire the habitude of all these virtues, I judg'd it would be well not to distract my attention by attempting the whole at once, but to fix it on one of them at a time; and, when I should be master of that, then to proceed to another, and so on, till I should have gone thro< the thirteen $[. ..] \ll^{23}$

Wie im folgenden Abschnitt ausführlicher behandelt, gedeihen erst Mitte des 19ten Jahrhunderts die gesellschaftlichen Bedingungen, die ein Selbst- und

22 Foucault beschreibt die Primärfunktion pastoraler Selbsttechniken entsprechend als Selbstzweck. Als »Präludium « (Foucault 2004[1978-1979]: 268) moderner Steuerungs- und Selbststeuerungsformen stehen sie zwar mit dem Subjekt der Couvernementalität in Verbindung, »dessen Verdienste auf analytische Weise identifiziert werden« (Foucault 2004[1978-1979]: 268f.), allerdings basieren sie dennoch darauf eine Folgsamkeit gegenüber religiös-moralischen Normen zu gewährleisten, indem sie gerade »den Ausschluss des Ich, den Ausschluss des Ego, den Ausschluss des Egoismus als zentrale Kernform des Individuums« (Foucault 2004[1978-1979]: 268) definieren, anstatt sich darauf auszurichten individuelle Freiheiten als Ressource der Staats- und Wirtschaftsentwicklung zu erschließen: »Was also das christliche Pastorat grundlegend und im wesentlichen charakterisiert, ist nicht die Beziehung zum Heil, nicht die Beziehung zum Cesetz, nicht die Beziehung zur Wahrheit. Das christliche Pastorat ist im Cegenteil eine Machtform, die, indem sie das Problem des Heils in ihre allgemeine Thematik aufnimmt, eine ganze Zirkulations-, Transfer-, Investitionsökonomie und -technik der Verdienste ins Innere dieses Cesamtverhältnisses schiebt, und dies ist ihr Hauptmerkmal« (Foucault 2004[1978-1979]: 266). In Bezug auf die Subjektivierende Funktion dieser Technologien bzw. einem eng an diesen Funktionen konzipierten Machtverständnis, überwiegen lange die Unterschiede zwischen Pastorat und (staatspolitischer) macht, ehe das Primat der ersteren mit Beginn der Moderne durch zweitere überformt wird. Obgleich es also "zwischen der pastoralen Macht der Kirche und der politischen Macht eine Serie von Interferenzen, von Stützen, von Relais, eine ganze Serie von Konflikten gibt [...], sodass die Kreuzung der politischen Macht tatsächlich eine historische Realität quer durch das Abendland ist«, ist mit Foucault ferner davon auszugehen, dass »die pastorale Macht trotz all dieser Interferenzen, trotz all dieser Kreuzungen, dieser Stützen, dieser Relais usw. in ihrer Form, in ihrem Funktionstypus, in ihrer internen Technologie wenigstens bis ins 18. Jahrhundert vollkommen spezifisch und verschieden bleibt von der politischen Macht« (Foucault 2004[1978-1979]: 226f.). 
Gesellschaftsverständnis in Zahlenform und in enger Beziehung zu Prozessen der Normalisierung, der Risikokalkulation, des Funktionalismus und der Kapitalisierung zunehmend $\mathrm{zu}$ einer zentralen Qualifikation in gesellschaftlichen Alltagspraxen erheben.

Obgleich die numerische Protokollierung Franklins aktuelle Formen der Selbstbeobachtung technisch in gewisser Weise vorwegnimmt, bedient sie vordergründig die christliche Konzeption des Verzichtes als tragende Säule der Selbstkonstituierung im okzidentalen Raum, die als primäres Subjektivierungsregime erst in den folgenden Jahrzehnten durch eine Begrenzung anhand des Kalküls der Nützlichkeit und einer Politik der Notwendigkeit verdrängt wird (Foucault 2004[1978-1979]): 94ff.). Auch wenn eine bedingte Vergleichbarkeit darin besteht, dass Franklin, wie auch die Self-Tracker*innen des 21ten Jahrhunderts, bei der eigenständigen Entwicklung der Technologien, die sie zur Umsetzung ihrer Subjektivierungsziele benötigen, auf protokollarisch-numerische Verfahren setzt, unterscheiden sich die Subjektivierungsziele damit dennoch deutlich durch ihre historische Bedingtheit.

Aus der genealogischen Perspektive erscheint das »Mining« der QuantifiedSelf-Mitglieder in den eigenen Datenspuren, demnach weder als ein Phänomen, das kausal aus historischen Vorgängerentwicklungen abgeleitet werden kann, noch als eine unbeeinflusst in Erscheinung tretende Innovation der Gegenwart. Es stellt weder die Krönung einer kontinuierlichen, technologischen Evolution, noch das spontane Produkt einzelner Genies dar, sondern ist ein diskursives Destillat, das an einzelne historische Denktraditionen anschließt und andere ignoriert. Diese Gemengelage aus verschiedenen politischen und technologischen Querbezügen soll im folgenden Abschnitt zumindest grob kartiert werden, ehe sie an eine Analyse gegenwärtiger (Selbst)Vermessungsphänomene angeschlossen wird. Die Analyse folgt dabei zunächst dem umfangreichen Forschungsstand, durch den die Zusammenhänge zwischen quantifizierenden Verfahren, die Genese des modernen Staates, der politischen Ökonomie sowie der Entstehung der Massenmedien (u.a. auf der Ebene diskursiver Aussagen) bereits vielfach diskutiert wurden. Die Darstellung bedient sich dabei einer argumentativen Trias, die für unterschiedliche historische Zeitpunkte einen primären Verdatungsmodus bestimmt, dies allerdings nicht um eine evolutionäre Chronologie moderner gesellschaftlicher Institutionen oder technologischer Innovationen nachzuzeichnen; vielmehr werden die verschiedenen Varianten der Verdatung als »Peripetie[n] der Regierung« (vgl. Foucault 2004[1978-1979]: 360) betrachtet, die zu einem bestimmten historischen Zeitpunkt als (vorläufige) Ergebnisse aus spezifischen Problemlösungsdiskursen hervorgegangen sind, diese dadurch aber gleichzeitig neu angefacht haben. 


\section{Die Analyse des Self-Tracking-Diskurses}





\section{Von Quetelet bis Quantified Self Statistische Regulation im soziotechnischen Wandel}

\subsection{Verdatung und Regulation - Von der Anthropometrie zur Sozialstatistik}

Die Erfassung der in einem Territorium oder Herrschaftsgebiet lebenden Menschen durch rudimentäre Auszählungen reicht geschichtlich weit zurück. Foucault beschreibt den Stellenwert, den quantifizierende Verfahren etwa für die Staatsräson einnahmen, bereits im Zusammenhang mit dem staatsphilosophischen Machiavellismus der Renaissance; wobei solche "protostatistischen « (Köhler 2008: 80f.) Verfahren aber auf militärische oder fiskalische Zwecke begrenzt waren (Behrisch 2006: 8).

Die Ursprünge der Statistik fallen historisch mit dem Ende der feudalistischen Gesellschaft zusammen. ${ }^{1}$ Als Methode der Herstellung übergreifender Verbindungen zwischen verschiedenen empirischen Elementen entwickelt sie sich in engem Zusammenhang mit der Ausdifferenzierung der politischen Ökonomie, die eine quantitative Beschreibung des Staates durch den Staat und für den Staat (Desrosières 2005: 165ff.) in einzigartiger Weise relevant macht. D.h. »die Statistik ist etymologisch die Kenntnis des Staates, die Kenntnis der Kräfte und Ressourcen, die einen Staat in einem gegebenen Moment charakterisieren « (Foucault 2004[19781979]: 396). ${ }^{2}$ Im Zusammenhang eines anhaltenden Diskurses verschiedener Wissenschaftsdisziplinen und staatlicher Organe konstituiert die Statistik des 18. und

Siehe hierzu auch Mämecke und Wehner (2014), sowie Mämecke (2016).

2

An anderer Stelle beschreibt Foucault die Entwicklung des Begriffs im Zusammenhang mit der Summe der Kenntnisse »vom Staat mit seinen verschiedenen Gegebenheiten, mit seinen verschiedenen Dimensionen, mit den verschiedenen Faktoren seiner Macht«, die »man als Wissenschaft vom Staat »Statistik« genannt hat« (Foucault 2004[1978-1979]: 152 [Hervorhebung im Original]). Desrosières verortet den Ursprung des Wortes im 18. Jahrhundert und beschreibt die Begriffsentstehung noch etwas genauer: „Die Staatenkunde war eine rein beschreibende Darstellung von »Staatsmerkwürdigkeiten«zum Gebrauch für Staatsmänner. Eine der Quellen für das Wort `Statistik « war das italienische Wort `statista〈, das `Staatsmann bedeutet« (Desrosières 2005: 22[Hervorhebungen im Original]). 
19. Jahrhunderts so nach und nach einen Äquivalenzraum unterschiedlicher Methodiken, Begriffe und Zeichen zur Beschreibung, Verwaltung und Gestaltung sozialer Prozesse (Desrosières 2005: 21, 84). ${ }^{3}$

Vor allem durch Foucaults Vorlesungsreihe »Staat, Territorium, Bevölkerung« gelangten Thesen zu großer Bekanntheit, die die "Geburt der Statistik« (Foucault 2004[1978-1979]: 369) und den Wandel der staatlichen Organisationsform als zirkuläre Prozesse wechselseitiger Beeinflussung und Abhängigkeiten beschreiben (vgl. Foucault 2001[1975-1976], 2004[1978-1979], 2004[1978-1979]). So haben die rasanten wirtschaftlichen und politischen Veränderungen mit dem Umbruch zur Moderne neuartige Probleme und Ungewissheiten hervorgerufen, für die sich die Statistik als Lösungsstrategie instrumentalisieren ließ. ${ }^{4}$ Angesichts der schwächer werdenden Legitimität der monarchistisch-territorialen Souveränität, die Foucault als Krise der Regierungskunst beschreibt, ermöglicht die statistische Emergenz neuer politischer Entitäten dem modernen Staat seine Legitimität auf die Notwendigkeit wirtschafts- und gesundheitspolitischer Interventionen umzustellen. ${ }^{5}$ Hierbei ist

»Exemplarisch für diese Auffassung sind die häufigen und lebhaften Debatten, die im 19. Jahrhundert zum Begriff des Mittelwertes, seines Status und seiner Interpretation geführt wurden« um durch diesen »schlüsselfertige Werkzeuge« herzustellen (Desrosières 2005: 77f).

Der Staat bietet zu dieser Zeit zwar sowohl die institutionelle Infrastruktur, als auch die Anwendungsszenarien für die Entwicklung der Statistischen Methode. Foucault siedelt sie letztlich aber beide auf der Ebene der Instrumente an und betrachtet sowohl den Staat, als auch die Statistik lediglich als »Peripetie der Regierung « (2004[1978-1979]: 360). Damit wird auch deutlich, dass Foucault, obwohl die Entwicklung der statistischen Verfahren und auch die Entstehung moderner Staaten und ökonomischer Strukturen an konkreten zeitlich und geografisch lokalisierbaren Beispielen beschreibt, dennoch das Ziel der Bildung verallgemeinerbarer Kategorien verfolgt, die auch über die von ihm gewählten Beispiele Cültigkeit für sich beanspruchen. Zudem wird auch deutlich, dass Konzepte wie Regierung (und damit ganz besonders auch das der Selbstregierung) zwar in unterschiedlichen historischen Kontexten aufgezeigt werden können, sich hier aber eben mindestens darin unterscheiden, dass sie sich für ihre Realisierung unterschiedliche Instrumente bedienen.

5 In der zehnten Vorlesung aus der Reihe »Sicherheit, Territorium, Bevölkerung«, in der er sich eingehender mit der Ceburt der Statistik auseinandersetzt, zitiert Foucault einen Zeitgenossen um die regelrechte Euphorie zu verdeutlichen, die mit diesen neu entstehenden politischen Interventionsinstrumenten einherging. Das Zitat macht aber auch die Ambivalenz zwischen Kontrollgewinn und Kontrollverlust deutlich, dass mit der (statistisch aufgezeigten) Notwendigkeit einhergeht. Da sie in sich selbst begründet scheint, sind ihr potentiell alle unterstellt: »So groß ist die Kraft der Notwendigkeit, dass sie wie eine souveräne Göttin, der in der Welt nichts heilig ist außer der Festigkeit ihrer unwiderruflichen Anhaltspunkte, alles Cöttliche und Menschliche ihrer Cewalt unterordnet. Die Notwendigkeit lässt die Gesetze verstummen. Die Notwendigkeit macht allen Privilegien ein Ende, um sich den Cehorsam aller zu verschaffen«Cardin Le Bret um 1600, zitiert nach Foucault (2004[1978-1979]: 380). Eine ähnlich argumentierende Weiterentwicklung dieses Themas findet sich in der durch Theo- 
in erster Linie an die Entität der Bevölkerung und die mit ihr verbundenen Potentiale und Probleme zu denken.

Die vormals der polizeilichen Administration überantworteten, rudimentären Auszählungen, ${ }^{6}$ entfalten sich an diesem Punkt zu einem der wichtigsten Instrumente liberaler Regierungsformen, in dem sie die juridischen Gesetze um die Gesetze der Statistik (Hacking 1990: 11) ergänzen: Dank »der Einbeziehung der spezifischen Probleme der Bevölkerung und dank der Absonderung jener Wirklichkeitsebene, die man Ökonomie nennt, [konnte] das Problem der Regierung schließlich außerhalb des juridischen Rahmens der Souveränität gedacht, erwogen und abgewogen werden [...]. Und so wird die selbe Statistik, die im Rahmen des Merkantilismus stets nur innerhalb und zugunsten einer monarchischen Administration funktionieren konnte [...] zu einem der Hauptfaktoren für die Aufhebung jener Blockade« (Foucault 2004[1977-1978]: 156). Im Zuge der fortlaufenden Transformation des Territorialstaates, werden vor allem Sozialerhebungen zunehmend als legitimierende Entscheidungsgrundlage in den Dienst der Staatslenkung gestellt (vgl. Foucault 2000: 64). Die Statistik ermöglichte es dabei die territorialen Grenzen, Komparabilitäts- und Konkurrenzrahmen zwischen den Ländern und Städten zu schaffen; die Verfügbarkeit von Ressourcen, sowie die Zirkulation der Güter und die Bevölkerungsentwicklung zu überwachen und längerfristiger Planung zu unterziehen. Darüber hinaus erzeugte aber auch die Umstellung auf kapitalistische Warenproduktion und Binnenhandel einen zunehmenden Bedarf an rational handhabbaren Wissensbeständen und Kalkulationsgrößen.

So waren es zu dieser Zeit vor allem Arbeitsämter, die als Erste umfassende Erhebungen zu Löhnen, Lebensbedingungen, Arbeitssicherheit, Hygiene, sowie Arbeits- und Streikhäufigkeit durchführten. Bald wurden aber auch in anderen Bereichen statistische Daten aggregiert, die als Nebenprodukte der amtlichen Verwaltungen anfielen (Desrosières 1999: 424): So z.B. durch die Abfertigung von Zöllen oder die Zentralisierung lokaler Gewerkschafts- und Versicherungsstatistiken

dore M. Porter unternommenen Analyse, nach der Statistiken eine Aura der Notwendigkeit erzeugen (Porter 1995).

6 »Daher ist der erste Gegenstand der Polizei, die Zahl der Menschen, die quantitative Entwicklung der Bevölkerung im Verhältnis zu den Ressourcen und den Möglichkeiten des Territoriums, das diese Bevölkerung bewohnt (Foucault 2004[1978-1979]: 466). Andrew Johnson, hat das sehr weitreichendes Konzept der Polizei in verschiedenen Schriften und Vorträgen Foucaults herausgearbeitet und verglichen. In Bezug auf die Vorlesungsreihe "Sicherheit, Territorium, Bevölkerung « resümiert er: »The police are the internal array of forces intended to modulate the conduct of domestic populations. Within this model, the police employ entirely different strategies related to public health, economic circulation and population management. [...] Police are responsible for quarantine programmes, the regulation of grain production and statistical accounting of population« (Johnson 2014: 12ff.). 
(Desrosières 2005: 175ff.; vgl. Hacking 1982: 282) oder durch die Ausstellung von Totenscheinen. ${ }^{7}$

Der Diskurs zentrierte sich allerdings zunehmend in den für die Erhebung, Aufbewahrung und Analyse der Daten geschaffenen statistischen Büros: »One could write the history of these bureaus as an aspect of the more general history of the construction of the state, insofar as they developed and legitimized a common language specifically combining the authority of science and that of the state (Desrosières 1999: 246). ${ }^{8}$

Anders als frühere rudimentäre Zählungen ist mit der systematischen Erhebung großer Datenbestände allerdings eine enorme realitätskonstruierende Wirkkraft verbunden (vgl. Hacking 1982; Porter 1994; Desrosières 2001; Espeland und Sauder 2007; Heintz 2010). Denn durch die Quantifizierung von einzelnen Einheiten oder Objekten wurden gesellschaftliche Strukturen und ihre Variationsbedingungen in bisher unbekanntem Maße sichtbar, allerdings auch in gleichem $\mathrm{Ma}$ ße konstruiert und als szientistische Elemente vergleichsweise einfacher Rechenoperationen abgeleitet. ${ }^{9}$ Der Übergang von nominalistischen Zählungen zu der

In seinem Aufsatz »Biopower And The Avalance Of Printed Numbers«, den lan Hacking selbst als Fußnote zu Foucaults Couvernementalitätsvorlesungen bezeichnet, schildert er anhand der ärztlichen Beglaubigungspraxis von Todesfällen beispielhaft ein solches zirkuläres Verhältnis zwischen empirischer Kategorienbildung und einer schleichenden Beugung der Empirie durch zunehmende Standardisierung: »No one would be allowed to die without a doctor signing a death certificate, but the causes of death « had to be uniform and easily applicable, in order that the certificates could be enumerated to discover who was dying of what, in what numbers« (Hacking 1982: 293).

8 Auch hier waren nicht nur die Methoden und Anwendungsfelder, sondern auch die epistemologischen Grundsätze ständiger Cegenstand diskursiver Kämpfe. Eine der Hauptkonfliktlinien verlief zwischen dem »environmentalism «- jener geografischen Häufigkeitsauszählungen, die die meisten statistischen Büros mit Kategorien wie Armut, Sterblichkeitsraten oder Epidemien in Beziehungssetzen um etwaige Wahrscheinlichkeiten regional zu spezifizieren und dem eugenischen »Aggregatrealismus«von Galton und Pearson, die die bevölkerungspolitische Ursachenforschung von der Räumlichkeit ins innere der Individuen verschieben wollten (Desrosières 1999: 246; 2005: 90). Die Astronomen Adolphe Quételet und Francis Galton versuchten Mitte des 19. Jahrhunderts in staatlichem Auftrag die Methoden des systematischen Vergleichs der körperlichen Beschaffenheit von Menschen (also die Jahrhunderte alte Technik der Anthropometrie) auf menschliches Verhalten zu generalisieren.

9 Cerade in ihrer Frühphase beflügelten die Experimente mit dem massenhaften Vergleich von Individuen und ihren Eigenschaften vor allem den wissenschaftlichen Realismus, dessen naturwissenschaftliche Annahmen nun die physikalische Natur eines durch Menschen gebildeten Cesellschaftskörpers (Gehring 2009: 99) bzw. die »Naturalität der Gesellschaft« selbst suchte (Foucault 2004[1978-1979]: 501). Ihre Faszinationskraft bezogen diese Zahlen aus den vermeintlich natürlichen Cesetzmäßigkeiten, die sich scheinbar allein durch die Quantifizierung von zählbaren Einheiten zutage fördern ließen. Diese neuen Perspektiven auf Bevölkerung und Individuum, die gleichsam mit ihrer Entstehung zusammenfallen, gruppieren sich dabei um die Normalverteilung, die als wichtigste Funktion der Wahrscheinlichkeits- 
Berechnung von Mittelwerten, führte $\mathrm{zu}$ einem sprachlichen und epistemologischen Shift, der aus Arbeitslosen Arbeitslosigkeit und Arbeitslosenquoten machte (Desrosières 2005: 78; Zimmermann 2006). Phänomene wie Warenzirkulation oder Volkswirtschaft erschlossen sich im gleichen Maße erst über den Beobachtungsrahmen massenhafter Einzelfälle, wie Kriminalität (Deflem 1997; Kreissl 2011: 54) und Gesundheit - bzw. der mit ihr verbundenen Subkategorien wie Alkoholismus, Suizid oder Verrücktheit (Hacking 1986: 222). Auch die Bevölkerung eines politischen Wirkungsbereiches erschien durch die Quantifizierung als Objekt mit eigenen Strukturen, Gesetzmäßigkeiten und Kennzahlen und wurde damit für regulative Zugriffe zugänglich (Köhler 2008: 82), womit sich an der veränderten Wahrnehmung der Bevölkerung eine empirische Bevölkerungspolitik gesundheits- oder wirtschaftsstrategischer Präventionen und Interventionen ausrichtete. Das zirkuläre Verstärkungsverhältnis statistischer Instrumente und sozialpolitischer Institutionen zeichnet sich demnach dadurch aus, dass Statistiken nicht nur die legitimierende Basis oder das Wissensressort für politische Strategien bilden, sondern sich in ihnen gleichermaßen die Erfolge der sozialpolitischen Maßnahmen widerspiegeln und sie darüber hinaus neue Interventionsfelder und damit auch neuen Bedarf an entsprechenden Regulationen aufzeigen (vgl. Muhle 2008: 245). Im Spiegel der Sozialstatistik erscheinen die individuell aleatorischen Merkmale wie Mortalität, Fertilität, Produktivität und Gesundheit fortan als kalkulierbare Größen. Dies hat zur Folge, dass sich Regulationstechniken des Regierens in diesem Zusammenhang dann nicht mehr nur auf den individuellen Einzelfall richten, sondern auf die Variationsbedingungen abstrakter Phänomene (vgl. Foucault 2004[1978-1979]: 157, 396).

Das innovative Moment der Sozialstatistik liegt demnach darin, dass sie den primären Modus des Einwirkens auf die Bewohner*innen eines Staatsgebietes unter den Bedingungen von Wohlfahrtstaatlichkeit von der Disziplinierung des Individuums auf die Regulation der Bevölkerung umstellt, d.h. »die Steigerung seiner Fähigkeiten, Ausnutzung seiner Kräfte, das parallele Anwachsen seiner Nützlichkeit und seiner Gelehrigkeit, seine Integration in wirksame und ökonomische Kontrollsysteme« (Foucault 1983[1976]: 135) gewährleistet.

rechnung (Hacking 1990; 2009), Mitte des 18. Jahrhunderts durch die kosmologischen Arbeiten von Moivre, Laplace und Gauß nachgewiesen (Desrosières 2005: 8; Desrosières 1999: 424f.), von Binet, Durkheim (2006[1897]) und Halbwachs auf das Soziale bezogen (Desrosières 2005: 78) und im Anschluss an die statistische Totalisierung des Quetelet'schen Durchschnittsmenschen (Cehring 2009; Desrosières 2005: 10) und die sozialdarwinistische Eugenik Galtons (Could 1983: 75ff.) etwa zur Bildung kriminal- oder »rassebiologischer« Kartografien verwendet wurde. Zur Cenealogie der »Vermessung des Verbrechers« siehe (Kailer 2011). Zur geschichtlichen Verbindung von »Volkszählung « und Nationalsozialistischer Ideologie siehe (Aly und Roth 2005[1984]). 


\subsection{Verdatung und Selbstregulation - Reflexive Sozialstatistik und Verbreitungsmedien}

Mit dem Übergang vom 18. zum 19. Jahrhundert wird die Verfügbarkeit des statistischen Blicks auf die Gesellschaft zunehmend aus der Ägide staatlicher oder wissenschaftlicher Institutionen genommen und z.B. in Form von Wahlstatistiken, Meinungsumfragen, Gesundheits- oder Sozialstatistiken öffentlich zugänglich, woran auch die zunehmende Entwicklung einer massenmedialen Öffentlichkeit maßgeblich beteiligt ist. ${ }^{10}$

Quantifizierungen etablieren sich zu dieser Zeit in viel breiterem Maße als universalistisches Selbstbeobachtungsverhältnis der Gesellschaft. Sie lassen z.B. den Staat nicht mehr nur als abstrakte Entität erscheinen, die außerhalb der Gesellschaft steht, sondern als Zusammenhang verschiedener Sozialbeziehungen (Desrosières 2005: 165), die die Repräsentant*innen politischer Ämter gleichermaßen umfasst wie ihre Wähler*innen. ${ }^{11}$ Und auch in anderen Bereichen, allen voran dem der Gesundheit, ermöglichen Statistiken die Selbsteinordnung, Kontrolle oder Abgrenzung im Rahmen des gesellschaftlichen Zusammenhangs. Für das moderne Subjekt ist daher charakteristisch, dass es sich in der Ambivalenz zwischen Masse und Individualität verortet. Über Statistiken bieten sich ihm erstmals Orientierungen in der Frage nach dem Zusammenhang von sich selbst und dem gesellschaftlichen Ganzen. Eine Frage, die mit der Moderne in einzigartiger Weise relevant wird.

Insbesondere durch die Normalismustheorie (Link 1997, 1999, 2001) wurden die Selbststeuerungskonzepte der Gouvernementalitätsforschung ${ }^{12}$ durch Untersuchungen $\mathrm{zu}$ normalisierenden Eigenschaften zahlenförmiger Sozialbe-

Im Zusammenhang mit der öffentlichen Verbreitung großer Zahlenaggregate gewinnt auch der Begriff Statistik mehrere Bedeutungsfacetten. So wird er im öffentlichen Gebrauch vor allem in Hinblick auf die verschiedenen Präsentationsformen statistischer Daten und weit weniger in Bezug auf die ihnen zu Grunde liegenden Methoden verwendet, die sich in den akademischen Fachdiskursen unter dem Begriff Statistik summieren: »For the public, and even for many people specializing in social studies, it designates numbers and measurements relating to the social world: population, gross national product, and unemployment, for instance. For academics in >Statistics Departments, $<$ however, it designates a branch of applied mathematics making it possible to build models in any area featuring large numbers, not necessarily dealing with society« (Desrosières 1999: 423[Hervorhebungen im Original]).

11 Zu der Frage, ob die Entwicklung der Demokratie der Verdatung voran ging oder ob die Verdatung als Pionier der Demokratisierung anzusehen ist siehe Link (1999: 164f.).

12 Nach dem Konzept der Gouvernementalität signalisieren Selbstbestimmung, Verantwortung und Wahlfreiheit »nicht die Grenze des Regierungshandelns, sondern sie sind selbst ein Instrument und Vehikel, um das Verhältnis der Subjekte zu sich selbst und zu anderen zu verändern« (Pongratz 2013: 226). 
schreibungen vertieft. ${ }^{13}$ Öffentliche Statistiken lassen sich hieran anschließend als eine Technologie beschreiben, die für eine Reintegration der Wahlfreiheit des individualisierten Subjekts in den Determinismus des Ganzen sorgt (vgl. Gehring 2009: 111f.). Im Zentrum dieser Form von Selbstregierung steht die Orientierung an öffentlich zugänglichen statistischen Durchschnitten, als vermeintlicher Spiegel der Normalität. Dieser Argumentation nach kommt der Normalverteilung die Doppelfunktion zu, dass sich gesellschaftliche Normalität nicht nur in ihr konsensualisiert (vgl. Bohn 2003), sondern sie darüber hinaus als normalisierender Rahmen auf die Gesellschaft zurückwirkt, da an der Orientierungsfolie des gesellschaftlichen Durchschnitts Selbstentwürfe gebildet werden, die durch die Abweichungsmaße und Extremwerte durchschnittlicher Verteilungen eingegrenzt werden (vgl. Link 1999: 10).

Mit der zunehmenden medialen Zugänglichkeit von Wahl- oder Bevölkerungsstatistiken lässt sich daher eine Verselbstständigung und Automatisierung der Steuerungseffekte von Bevölkerungsstatistiken ausmachen, wobei die Mechanismen der Selbststeuerung sukzessive die direkten politischen Interventionen ergänzen und gekoppelt mit einem eigendynamischen und nicht deterministischen Gesellschaftsverständnis repressives Regierungshandeln in eine vielseitige Steuerung sich selbst steuernder Entitäten transformiert.

Die ehemals durch den »Aggregatrealismus« (Desrosières 2005: 90) der frühen Statistiker konstatierte Normalgesellschaft erweist sich dieser Argumentation

13 »Es handelt sich dabei um nichts anderes als um die scheinbar viel banalere Feststellung, dass der interdiskursive Komplex der Normalität durchgängig an quantifizierbares statistisches Wissen gekoppelt ist. Ich spreche wegen der strategischen Sonderstellung dieses Komplexes in modernen Diskurssystemen und wegen seiner enormen Bedeutung vom Komplex des »Normalismus «. Erstmals wird hier spezialdiskursiv erarbeitetes Expertenwissen (statistische Daten und statistische Trends in Form von Tabellen und Kurven) en gros « konstitutiv für gesellschaftliche Subjektivierungsprozesse, in dem es als vermeintliches Wissen über große schweigende Mehrheiten im medialen Diskurs verbreitet wird (Link 2001: 81[Hervorhebung im Original]). »Der Kern dieses Expertenwissens beruht auf der normalistischen Statistik, die mit Statistik allgemein nicht deckungsgleich ist. Normalistische Statistik beruht auf der Cenerierung normalistischer sozialer und psychischer Objekte auf der Basis von massenhafter Verdatung in homogenen Normalfeldern, auf Eindimensionalisierung, Skalierung und Quantifizierung [...]. Berühmte Beispiele normalistisch generierter sozialer und psychischer Objekte sind: »Leistung«, »Intelligenz«, [...] »Cesundheit«, bzw. negativ »Stress« [...] Jedes dieser Objekte erstreckt sich innerhalb eines »eindimensional « gerichteten Normalfeldes, wobei eine oder typischerweise zwei symmetrische Normalitätsgrenzen die Zonen der Normalität und der Anormalität trennen. «Diese Grenzen sind meist dynamisch bzw. flexibel auf einem graduierten Kontinuum verschiebbar. »Das ergibt sich aus dem statistischen Verfahren: wenn eine »Population « unter einem bestimmten Cesichtspunkt verdatet wird, wird sie dadurch homogenisiert, ihre Verteilung um den Durchschnitt (die symbolische »Mitte«) zeigt zwar»Wendepunkte«der Gaußschen »Normalverteilung«, aber niemals qualitative Diskontinuitäten« (Link 2001: 82f.[Hervorhebungen im Original]). 
folgend daher eher als Normalisierungsgesellschaft im Sinne einer sich selbst erfüllenden Prophezeiung, indem die Normalität an die Stelle von Normativität als gesellschaftsuniverselle Wertbindung tritt.

\subsection{Selbstverdatung und Selbstregulation - Statistik und interaktive Internetmedien}

Wo die aktuelle Grenze zwischen Normalität und Anormalität zu einem spezifischen Zeitpunkt liegt, ergibt sich dabei als Wechselspiel zwischen statistischer Distribution aktueller und vergangener Normalitätsgrenzen ${ }^{14}$, ihrer diskursiven Verteidigung und Infragestellung sowie der erneuten Erhebung und Veröffentlichung dieser Dynamiken in Form statistischer Momentaufnahmen (vgl. Link 2001: 83).

Mit diesem Zusammenspiel von Datenerhebung und Datenveröffentlichung ist eine sich selbst immer wieder erneuernde und in Schwung bringende Spirale der Beobachtung und Erzeugung von Normalverteilungen in Gang gesetzt worden (Gehring 2009: 112), die sich unter den technologischen Bedingungen des Internets nun weiter verengt. ${ }^{15}$ Mit dem Hinzutreten von interaktiven Internetmedien als Technologien der Erzeugung und Vermittlung von Statistiken werden über die Zugänge zu statistischen Daten hinaus inzwischen auch die Erhebungsmittel dezentralisiert. Analytisch lässt sich damit eine dritte Verdatungsphase unterscheiden, die am treffensten als Selbstverdatungsphase beschrieben ist. Das umfangreiche Repertoire an verschiedensten Internetportalen, Apps oder anderen tragbaren Sensoren, die auf unterschiedliche Weise Körperfunktionen aufzeichnen, Bewegungsprofile erstellen, Daten über den Umgang mit Ressourcen wie Geld oder Zeit aufzeichnen, wird inzwischen vielfach für die Erstellung von Finanz-, Therapie-, Diät-, Trainings- oder Arbeitsplänen verwendet.

Während der letzten zehn Jahre hat sich so ein außerordentlich lebhaftes Experimentierfeld technologieaffiner Subkulturen entwickelt, deren Mitglieder der Antrieb zu vereinen scheint, sich selbst systematisch beobachten oder verändern $\mathrm{zu}$ wollen. Die hierfür aufgewendeten Methoden reichen von den Versuchen einer lückenlosen Echtzeiterfassung des eigenen Lebens durch kleine Kameras und

14 So sorgt moralische und juridische Normativität für die Attraktionskraft der Verteilungsmitte und die Repulsionskraft der äußeren Extreme einer Gaußkurve, in dem sie gesetzliche Grenzen setzt oder durch diskursive »ko-situieriung « normative Narrationen »konnotativ an die statistischen Tableuas« anschließt (vgl. Link 2001: 90f.).

15 Für aktuelle Beispiele massenmedialer, interaktiver Inforgrafiken, Kurven ikonografischen Illustrationen im Zusammenhang mit interaktiven Internetmedien siehe den Sammelband »Quoten Kurven und Profile - Zur Vermessung der sozialen Welt« (Passoth und Wehner 2013). 
tabellarische Verhaltensprotokolle bis zur Genomsequenzierung und dem inversiven Enhancement der radikal posthumanistischen Body-Hacking-Szene. Über diese Extreme hinaus ist allerdings vor allem eine zunehmende Verbreitung von Wearable Technologies, in Form von Uhren, Gürtelclips oder Armbändern und eine exponentielle Evolution von Sensoren, Interfaces und Smartphone-Apps zu verzeichnen, die weitaus niedrigschwelligere Praktiken der zahlenbasierten Selbstbeobachtung bis heute soweit popularisiert haben, dass sich von einem Phänomen von gesellschaftlicher Tragweite sprechen lässt.

Der vorangegangene wissensgeschichtliche Abriss sollte deutlich machen, dass die Konstituierung des modernen Selbst seit jeher in starkem Maße mit numerischen Messwerten und vor allem öffentlichen Statistiken koinzidiert. Über die Frage hinaus, worin sich die gesellschaftliche Bedeutung von numerischen Formen von Selbst- und Gesellschaftsverstehen wissensgeschichtlich begründet, soll sich im Folgenden nun dezidierter dem Umstand zugewendet werden, dass gegenwärtig eine Vielzahl von zahlenbasierten Selbstbeobachtungs- und Vergleichstechniken dezentral und autark entwickelt, erprobt und angewandt werden. Galt der Fokus des vorangehenden Abschnitts der Bemühung die konstitutive Bedeutung zahlenförmiger Beobachtungsverhältnisse für die Gesellschaft im Allgemeinen zu resümieren, sollen nun Erklärungen dafür gesucht werden, dass sich das Phänomen der zahlenförmigen Selbstbeobachtung gegenwärtig zu einem Phänomen der progressiven Selbstverdatung weiterentwickelt.

Im Anschluss an die Überlegungen aus dem vorangehenden Abschnitt gilt besondere Aufmerksamkeit dabei der Frage danach, an welche Traditionen-Wissensreservoirs, Argumentationsmuster und Denkschulen dabei angeschlossen und mit welchen im besonderem Maß gebrochen wird. Zudem sollen Selbstauferlegte Ansprüche der Befreiung systematisiert, hinterfragt und in Beziehung gesetzt werden zu einer kritischen Theoretisierung von Freiheitsbegriffen und den Ambivalenzen von Selbstermächtigungsprozessen. 



\section{Self-Tracking im Schnittfeld progressiver Technologiekulturen und Kreativitätsdispositive}

Das Self-Tracking kann damit nur als eine von vielen Formen der selbstreferenziellen Analyse und Modifizierung gelten, die sich während der letzten Jahre inmitten einer kontinuierlichen Genese materieller und digitaler Technologiekulturen etabliert haben. Dies zeigt sich nicht zuletzt auch daran, dass die verschiedenen Praktiken und Teildiskurse technologiebasierter Selbstexploration wie z.B. die Genomsequenzierung oder das inversive Enhancement der radikal posthumanistischen Body-Hacking-Szene, videobasiertes Lifelogging oder numerisches SelfTracking keinesfalls überschneidungsfrei entstanden. Gerade in der Anfangsphase lassen sich zwischen verschiedenen Ansätzen gleichermaßen Anziehungs- und Abstoßungseffekte im Zusammenhang mit community building sowie der Besetzung spezieller Begriffe und Praktiken ausmachen. ${ }^{1}$

»To overcome these limitations, Self-tracking applications technologies are needed, which enable optimization beyond the limits. Quantified Self can therefore be seen as a means to an end for the transhumanist project.«[sic! $]^{2}$

Über thematische, personale oder rhetorisch/semantische Parallelen hinaus gleichen sich diese verschiedenen Subkulturen zudem in ihrer Ablehnung von normierter Konsumtion und einer neoliberalen Akzentuierung der Unabhängigkeitsideale ästhetisch-ethischer DIY-Kulturen - womit die Entwicklung auf einen Phänomenbereich stößt, der als Bedeutungsverlust traditioneller Gemeinplätze hegemonialer Subjektordnungen (wie z.B. des affirmativen Massenkonsums und büro-

Die Indifferenz mit der Begriffe wie »Quantified Self« und »Posthumanismus« bis heute auch in den Sozial- und Kulturwissenschaften verwendet werden, zeugt ebenfalls von der Fluidität des Selbstvermessungsdiskurses. Siehe dazu z.B. die Vortragsfolien von Ramon Reichert - The Quantified Self in the Era of Digital Posthumanism, 15th May 2014. "Quelle: www.academia.edu/8853717/The_Quantified_Self_in_the_Era_of_Digital_Posthumanism_15t h_May_2014_Humanism_Culture_or_Illusion_International_Scientific_Conference_Faculty_ of_Philology_Belgrade_University (zuletzt aufgerufen am 01.06.2016).

2 Quelle: http://indiafuturesociety.org/quantified-self-and-transhumanism-a-means-to-an-en d/ (zuletzt aufgerufen am 02.09.2015). 
kratischer Normalarbeitsverhältnisse) schon umfangreich theoretisiert wurde. Neben Begriffen wie Innovations- oder Risikogesellschaft, Prosumption, Prekarisierung, Postoperaismus oder den marxistisch verwurzelten Postfordismus-Theorien, scheinen hier vor allem verschiedene Konzepte postmoderner Subjektkulturen gesellschaftliche Entwicklungen zu beschreiben, die dem Self-Tracking und anderen technologiebasierten Selbstformungsprojekten in vieler Hinsicht vorgängig sind und ihre Selbstbeschreibung als avantgardistische Pionierkultur damit relativeren. Angefangen mit dem Unternehmer seiner selbst (Foucault 2010), über das unternehmerische Individuum (Rose 2000) und das unternehmerische Selbst (Bröckling 2007) bis hin zum ästhetischen Kultursubjekt (Reckwitz 2010) wurden verschiedene Varianten bereits ausführlich beschrieben, mit denen das Postulat des Neuen und der unabschließbaren Selbsterfindung als ehemals »elitäre und oppositionelle Orientierung am Kreativen « inzwischen »allgemein erstrebenswert und zugleich für alle verbindlich« geworden ist (Reckwitz 2011: 16).

Mit der Subjektivitätsforschung der jüngeren Kultursoziologie lassen sich offensichtliche Merkmale des Self-Tracking, wie z.B. seine Projektförmigkeit oder verschiedene Effizienz- und Optimierungsimplikationen somit zeitdiagnostisch rahmen. Die Ausweitung des Analysefokus auf die spezifischen Eigenheiten gegenwärtiger Gesellschaftsentwicklungen, die Bröckling als Ökonomisierung der Kultur und Reckwitz im weitesten Sinne als Kulturalisierung der Ökonomie beschreiben, eröffnet dabei die Möglichkeit das Phänomen des Self-Tracking losgelöst von der Selbstbeschreibung der Quantified-Self-Community, als einen spezifischen gesellschaftlichen Ausdruck zu skizzieren, der einerseits aus der andauernden Tradition zahlenförmiger Beobachtungsverhältnisse der Gesellschaft sowie andererseits aus den individualisierenden ökonomischen Anforderungen und Kreativitätsimperativen erwächst, die in den 1970er Jahren hinzutreten.

Foucaults Bestrebungen folgend, mit der Analyse des Archivs gesellschaftliche Ereignisse auf ihr Entstehen, also auf die Prinzipien und Gesetzmäßigkeiten ihres Erscheinens, hin zu prüfen (Lüders 2007: 95), liegt der Fokus der folgenden Abschnitte zunächst noch weniger auf der Frage welche Subjekttransformationen durch Selbstvermessungstechnologien angeleitet werden, sondern vielmehr auf dem Versuch das Subjektverhältnis zu konturieren, vor dessen Hintergrund die Praktik der Selbstvermessung überhaupt als eine naheliegende Idee erscheint. Für die Skizze dieses Subjektverhältnisses werden über die weitreichenden immateriellen (diskursiven) Bedingungen hinaus auch nicht-diskursive Bedingungen beleuchtet (vgl. Foucault 1981[1969]: 234), in denen sich institutionelle Wissensformen mit Machttechnologien zu ökonomischen oder rechtlichen Zwängen verbinden. Hierbei erscheinen jüngere Phänomene, die gemeinhin unter den Begriffen »Prekarisierung« und »Kreativwirtschaft« verhandelt werden, von besonderem Interesse $\mathrm{zu}$ sein. 
In der gesellschaftlichen Verallgemeinerung von Kreativitätsidealen und intrinsischer Begeisterungsfähigkeit für die eigenen Tätigkeiten, nimmt darüber hinaus die Figur der Künstlerin eine zentrale Rolle ein. Die Veränderung ihrer gesellschaftlichen Bedeutung lässt sich dabei nicht ausschließlich anhand der diskursiven Verschiebungen der entsprechenden Semantiken beschreiben, vielmehr finden sich auch in der soziologischen Ungleicheitsforschung Analysen, in denen diese Entwicklung kongruent auf der Ebene sozialpolitischer Reformen untersucht wird. Zwar reicht ihr Erklärungsanspruch streng genommen über die methodische Selbstbeschränkung der diskursanalytischen Subjekttheorie hinaus, die keine gesellschaftlichen Ist-Zustände erklären will sondern diese als dominante Vorstellungen von Realität selbst problematisiert, allerdings lassen sich ihre Dokumentenanalysen oder Interviews durchaus in die Beschreibung einfügen, ohne den Bereich objektivistischer Epistemologie zu berühren. Hier stellen insbesondere die Analysen von Alexandra Manske eine Brücke zwischen der subjektorientierten Arbeitssoziologie (so wie sie populär etwa durch Günter Voß vertreten wird) und der poststrukturalistischen Subjekttheorie her. ${ }^{3}$ D.h. trotz eines mitunter essentialistisch anmutenden Subjektbegriffs, der sich so allerdings auch sehr deutlich bei Reckwitz findet, ${ }^{4}$ lassen sich auch die durch sie untersuchten administrativen Strategien, Gesetzesdebatten und Enquete-Berichte (ganz ähnlich zum Vorgehen der Gouvernementalitätsstudien) als Schauplätze und Vergegenständlichungen eines Mechanismus verstehen, der eine spezifische Ordnung der Realität gleichzeitig voraussetzt und durch entsprechende Regulationstechniken in Bezug auf diese "Realität« erst konstituiert (vgl. Bröckling und Krasmann 2010: 24ff.).

Entsprechend besteht das Ziel der folgenden Abschnitte darin, eine theoretische und begriffliche Basis zu schaffen, auf die sich in der Analyse von Selbstver-

3 Indem Manske den Begriff »unternehmerisches Selbst« dem stärker arbeitssoziologisch konnotierten Äquivalent des »Arbeitskraftunternehmers « vorzieht, bezieht sie sich indirekt auf die diskursanalytischen Subjekttheorie: »Anhand einer Analyse themenspezifischer, politischer Diskussionen und wohlfahrtsstaatlicher Instrumente wird gezeigt, dass der wohlfahrtsstaatliche Paradigmenwechsel in der sozialpolitischen Absicherung von künstlerischkreativ Erwerbstätigen erstens einen Wandel sozialpolitischer Zielvorstellungen beinhaltet und im Kern dem Leitbild des sozialpolitisch zu aktivierenden, unternehmerischen Selbst folgt« (Manske 2013: 261). Ihr Zugriff auf das Thema lässt sich insofern als hybride Untersuchung diskursiver und materialistisch ausgelegter nicht-diskursiver Faktoren verstehen.

4 Sowohl bei Manske als auch bei Reckwitz wird immer wieder der Eindruck erweckt, sie würden den Begriff »Subjekt« synonym mit dem Begriff»Individuum« verwenden. Die Verwendung des Wortes »Subjekt« im Plural als »Subjekte« kann als ein konkreter Hinweis für eine unklare Positionierung gegenüber Aussagen über eine konkrete Gruppe von Menschen einerseits und gesellschaftlichen Leitbildern andererseits gelesen werden. Insbesondere da der Singular »Subjekt « im Grunde besser geeignet ist um ein eine Zielprojektion von Selbsttransformationsprozessen zu beschreiben. 
messungstechnologien und ihrer diskursiven Herstellungsprozesse zurückkommen lässt, ohne dass dabei die Emergenz dieser Technologien schlicht als praktische Reaktion auf gesellschaftliche Diskurse oder kausale Folgen z.B. einer sich verändernden Sozialstaatspolitik betrachtet werden.

Nach Reckwitz' genealogischer Rekonstruktion verschiedener Subjekttransformationsprozesse in der Moderne, beginnen sich mit den 1970er und 1980er Jahren in Form neuer Modi der lebensstilorientierten Konsumtion sowie sportlicher und gesundheitsbezogener Körperpraktiken verschiedene Modi der Selbsteinwirkung herauszubilden, die sich von früheren Selbstpraktiken durch ihren Grad an Selbstreferentialität und ihre Nähe zu digitalen Technologien abheben (Reckwitz 2010: 555). Sie entwickeln sich im Rahmen eines "Californian way of life« (ebd.) zunächst in den wissensintensiven Arbeits- und Konsumtionsmillieus westlicher Postindustrienationen, deren gesellschaftspolitische Bedeutung der Ökonom Richard Florida mit provokanten Anleihen an marxeologisches Vokabular durch das Label der "Creative Class « $\mathrm{zu}$ internationaler Wahrnehmung verholfen hat (Florida 2002). Wie Reckwitz anmerkt, bleiben diese Formen der Selbststilisierung anhand von digitalen Schaffensakten dabei nicht auf den Kreis lokaler Kulturen beschränkt, sondern gelangen bald zu einer hegemonialen Attraktivität mit potentiell gesellschaftsweiter Strahlkraft, ehe sie sich schließlich als fester Bestandteil einer postmodernen Subjektordnung etablieren. Diese Thesen werden nicht zuletzt durch zahlreiche Analysen von Werbebildern gestützt, die als populäre Formen visueller Diskursbeiträge u.a. Hinweise darauf liefern, wie sich in den 1970er und 1980er Jahren Vorstellungsbilder und Wertvorstellungen in Bezug auf Arbeit »(re)inszenieren «, indem sie einen grundsätzlichen Wandel von der Produkt- zur Imagewerbung kenntlich machen (Barth 2009: 186). Dieser Kontrast kennzeichnet eine zunehmend unschärfer werdende Grenze (Reckwitz 2010b) zwischen Konsumtionsprodukten und persönlicher Identität, die sich durch die individuelle Verwendung insbesondere von digitalen Technologien gewinnen und zum Ausdruck bringen lässt.

»Das individuelle Streben nach Glück verlagerte sich auf die Sphäre des Konsums und dieser versprach nicht länger die serielle Befriedigung normierter Bedürfnisse im Rahmen fordistischer Massenkultur, sondern lockte mit Abenteuer und Selbstverwirklichung und ließ materielle Ungleichheiten im Lobpreis der Differenz verschwinden. Konsumistischer und unternehmerischer Imperativ fielen zusammen: Als Konsument sollte der Einzelne sein Genusskapital akkumulieren und hatte sich zu diesem Zwecke so innovativ, risikobereit und entscheidungsfreudig zu erweisen, als müsse er ein Unternehmen zum Markterfolg führen« (Bröckling 2007: 51[sic!]). Wie Bröckling in Bezug auf den französischen Publizisten Paul Thibaud schreibt, konnten hier über den elaborierten Individualkonsum auch profitorientierte Verhaltensdispositionen entwickelt werden: »Von einem Unterneh- 
mer im Dienste des eigenen Genusses kann man zu einem Unternehmer im Allgemeinen werden « (Thibaud 1985: 134). ${ }^{5}$

Insbesondere die zu dieser Zeit aufkommende Anpreisung von Computern für den Heimbereich verheißt über den Besitz von PC und Mac Individualität, Selbstverwirklichung und Kreativität im Zusammenhang mit einer Emanzipation von der fremdbestimmten, hierarchisch strukturierten und bürokratisch organisierten Arbeitswelt (Barth 2009: 188ff). »Üblicherweise konnotiert Werbung [...] Glück durch die Re-komplettierung des Subjekts über ein Konsumgut, dessen Warenaneignung die Prozesse der Enteignung und Verausgabung in der Produktion zu heilen vorgibt. Computer, deren Abbildungen Glück als Sicherheit, als Genießen, als Überschreitung, als Ermächtigung etc. herstellen sollen, haben nicht nur Warencharakter, sondern sind eben auch Produktionsmittel, womit sich die Konnotation des Glücks auf eine Produktionsweise erstreckt « (Betz und Riegler 2003: 130f.). ${ }^{6}$ Mit der Verbreitung zweckmäßig nicht mehr eindeutig bestimmter digitaler Produktionsmittel wie PC und Mac wurde zu diesem Zeitpunkt zunächst die materielle Basis für eine branchenübergreifende Individualisierung von Erwerbsarbeit geschaffen. Die hieraus resultierende Welle von Firmengründungen fand ihren Höhepunkt in der New Economy der 1990er Jahre, die ihr Selbstverständnis aus der bewussten Abgrenzung zur Risikoscheu und Traditionsverhaftetheit der Old Economy bezog (Barth 2009: 188ff.). Auch im deutschsprachigen Raum bringt der Diskurs bis heute immer wieder Manifeste hervor, die eine übergreifende Identität, ganz im Sinne von Floridas Klassenanalogie, als progressives Kollektivbewusstsein zu initiieren versuchen. ${ }^{7}$ Holm Friebe und Sascha Lobo werten mit dem vergleichsweise aktuellen Pamphlet »Die digitale Bohème« aus dem Jahr 2006 etwa »Festangestellte« als Auslaufmodelle $\mathrm{ab}$ und konstruieren die vermeintliche Unflexibilität, Konformität, Unmündigkeit und das Sicherheitsdenken konventioneller Arbeitnehmer*innen als Gegenpol zur kompromisslosen Risikofreudigkeit, Kreativität und Freiheit der Alleinselbstständigen in posttraditionalen Arbeitsfeldern (Barth 2009: 183).

»Wenn wir Bohème sagen, sprechen wir von einer Cruppe, die ihr Schicksal arbeitstechnisch in die eigenen Hände nimmt und dabei mehr Wert auf Selbstop-

$5 \quad$ Zitiert nach der deutschen Übersetzung durch Ulrich Bröckling (2007: 51).

$6 \quad$ Zitiert nach Bath (2009:188).

7 Obgleich die Ungleichheitsforschung von dem Klassenbegriff sowohl als horizontales Schichtungsmodell sowie als Konzept eines politischen Kollektivbewusstseins spätestens mit den Schriften von Bourdieu, Castel, Schultheiß und Lessenich abgelassen hat und sie in einer soziologisch unterfütterten Milieutheorien hat aufgehen lassen, erfährt der Begriff hier in nahezu ironischer Weise wieder Konjunktur-als überindividuelle Selbstthematisierung im Zusammenhang mit einer Politisierung von Arbeitsbedingungen. Wohingegen der Begriff im marxistischen Vokabular die strukturelle Ausbeutung der Arbeitenden kennzeichnet, wird er heute von den Arbeitenden der Kreativbranchen verwandt, um strukturelle Hemmnisse für die optimale Selbstausbeutung kollektiv zu thematisieren. 
timierung und individuelle Freundschaften legt als auf karrierefördernde Anpassung. $\ll^{8}$

»Im sich herausbildenden Nachfolgemilieu der alten Angestelltengesellschaft « (Reckwitz 2011: 504), das die rigiden Normalarbeitsverhältnisse schrittweise ablöst, diffundiert damit einerseits ein Verständnis von Arbeit als Ort der Selbstverwirklichung, das sich in libidinös besetzten Idealisierungen künstlerischer, eigenständiger Tätigkeiten begründet und das anderseits auf eine tiefgreifende strukturelle Veränderung in den Sozialsystemen moderner Staaten trifft, die ihrerseits das Ende der Normalarbeitsbiografie und kollektiver Wohlstandsversprechen einläuten.

Beginnend mit dem »Thatcherism« über die »Reaganomics« Ende der 7oer und Anfang der 80er Jahre bis zur »Agenda 2010« wurde die marktradikale Wende vieler westlicher Staaten von einer Schwerpunktsetzung staatlicher Interventionen zugunsten der Etablierung einer enterprise culture durchzogen (Bröckling 2007: 53), die vor allem ideenreiche, eigeninteressierte Arbeitnehmer*innen prämiert (Reckwitz 2011: 507) und sie mehr oder minder unvermittelt mit den Gesetzmäßigkeiten der Marktwirtschaft kurzschließt. Die hieraus erwachsende postbürokratische und vornehmlich projektförmig organisierte Arbeitskultur präjudiziert fortan ein Subjekt, das sich in erster Linie als eine Verschmelzung aus »Kreativsubjekt « und »unternehmerischem Subjekt« darstellt« (Reckwitz 2011: 500[Hervorhebung im Original]) und das über innere und äußere Erfolgsfaktoren beständig reflektiert. Im Übergang vom Wohlfahrtsstaat zum aktivierenden Staat triumphiert daher die Figur der Entrepreneurin (vgl. Thibaud 1985: 134ff.) als Produkt jener Bestrebungen, ein "weiter steigendes Leistungsniveau der ökonomischen Aktivitäten eines Landes, seiner Politik und seiner Regierung, der Künste und Wissenschaften und auch der individuellen Lebensführung seiner Bewohner [zu] befördern. «9

\subsection{Interdiskursbeziehungen: Technologieinnovation und Kreativwirtschaft}

Wohingegen Künstler"innen und Kulturschaffende insbesondere in Deutschland zunächst in der bildungsbürgerlichen Tradition romantischer Künste eine gewisse Sonderrolle zugeschrieben wurde, die auch ihren Status im Zusammenhang mit wohlfahrtsstaatlichen Agenturen mitbestimmte, begann in den 7oer Jahren ihre sozialpolitische Eingliederung in den Arbeitsmarkt und die sozialen Sicherungssysteme. Ihre sozialpolitische Inklusion erfolgte hier vornehmlich in Abhängigkeit

8 (Friebe und Lobo 2006: 28f.).

9 Das Zitat stammt aus einem Dokument des britischen Centers für Policy Studies, das Bröckling (2007: 54) nach Paul Morris (1991: 23) zitiert. 
zu einem nachweisbaren erwerbswirtschaftlichen Erfolg, womit der traditionelle Sonderstatus künstlerischer Tätigkeiten bereits zu erodieren begann (Manske 2013: 262f.). Im Verlauf der 1980er Jahre erscheinen die Bereiche Kunst und Wirtschaft diskursiv dann immer weniger als zwei voneinander getrennte Bereiche mit eigenen Gesetzmäßigkeiten, ehe im Zuge der allgemeinen Liberalisierung des wohlfahrtsstaatlichen Diskurses schließlich eine markante Akzentverschiebung in der Thematisierung von Künstler*innen stattfindet. So werden sie zunächst als Kulturdienstleister*innen und dann in universalisierter Form als "Kreative « adressiert, womit bereits eine Passung an den Begriff »Kreativwirtschaft« hergestellt wird, der sich schrittweise zu einem hervorstechenden semantischen Bezugsrahmen unterschiedlichster Formen von künstlerisch-kreativer Arbeit herausbildet (Manske 2013: 267). Triebfedern dieser wirtschaftspolitischen Akzentuierung von künstlerisch-kreativer Arbeit sind vor allem die seit den 1990er Jahren regelmäßig durch Kulturwirtschaftsberichte, mit denen die Beobachtung und Berichterstattung der Soziallage von Künstler*innen in Deutschland aus der Ägide des Arbeitsministeriums direkt in das Wirtschaftsressort übertragen wurden. Eine strukturelle Veränderung, die vor allem die Inszenierung der Kreativwirtschaft als Kernbereich der postfordistischen Ökonomie durch die Verbindung von technischen Fortschrittsszenarien mit Innovationen auf kultureller Ebene vorantreibt (vgl. Reckwitz 2011: 140).

In zahlreichen Enquete-Berichten und BMWi-Reports wird die Kreativwirtschaft dabei zum Innovationsmotor und zur Zukunftsbranche stilisiert die zukünftig noch weiterreichende volkswirtschaftliche Impulse in Aussicht stelle. Indem gleichen Maße indem die Berichte die Bereiche Kunst und Kultur dahingehend problematisieren, dass sie sich lediglich sich selbst gegenüber verpflichtet seien, führen sie ihre zunehmende Ökonomisierung als bevorzugten Modus an, um ihre gesamtgesellschaftliche Integration $\mathrm{zu}$ beschleunigen (Manske 2013: 267).

Die normative Leitlinie künstlerisch-kreativer Arbeit wurde schließlich mit der New-Labour-Politik und den sozialdemokratischen Reformdiskursen Ende der 1990er Jahre weiter liberalisiert. Exemplarisch stehen hierfür vor allem das sog. "Schröder-Blair-Papier«, deren Quintessenz sich im deutschsprachigen Diskurs in der Losung »Fördern und Fordern« manifestierte. Damit wurde erstmals ein systematischer, begriffstheoretischer »Baukasten für eine semantische Rekonstruktion künstlerisch-kreativer Arbeit« unter dem Label Creative Industries angeboten, der eine steigende wirtschaftspolitische Bedeutung von Kunst und Kultur beschwor, sie gleichzeitig jedoch auch an vergleichsweise junge Segmente der boomenden Technologiebranche und der Knowledge Economy anschloss und von der bisher dominanten Strategie eines öffentlich geförderten Kultursektors trennte (Manske 2013: 266). Im Jahr 2001 erhielt das Konzept Creative Industries durch das »Creative Industries Mapping Document « vom Department for Culture, Media and Sport der New-Labour-Regierung seine bis heute geltende Definition. Ihr nach zählen 
$\mathrm{zu}$ den Creative Industries »those industries which have their origin in individual creativity, skill and talent and which have a potential for wealth and job creation through the generation and exploitation of intellectual property. $\aleph^{10}$

Kreativität wird damit in einem doppeten Sinne zum Fluchtpunkt eines wirtschaftspolitischen Diskurses sowie nicht-diskursiver Maßnahmen. Einerseits indem anhand verschiedener sozialpolitischer Arrangements oder durch die Initiation typischer gouvernementaler Agenturen der neuen sozialstaatlichen Aktivierungsprogrammatik die Ausübung kreativer Tätigkeiten institutionell und rechtlich stärker mit den Mechanismen der Wertschöpfung verwoben werden. Und andererseits indem »Kreative« als soziale Avantgarde diskursiv idealisiert werden, die Kraft schöpferischen Talents und Eigenständigkeit wirtschaftlichen Aufschwung bewirken (Manske 2013: 269f).

Während die Arbeitsfelder der Künstler*innen ökonomisiert und wettbewerbsförmig reorganisiert werden, bzw. der Formwandel wohlfahrtsstaatlicher Institutionen auch in diesem gesellschaftlichen Bereich die universalen Integrationsansprüche sozialer Absicherung aufhebt und sie analog $\mathrm{zu}$ den Prinzipien selbstständiger Erwerbsarbeit neukonstruiert, sollen etwa betriebswirtschaftlich ausgerichtete Coaching-Angebote »Kreative« bei der Verbesserung ihrer Erwerbschancen im Hinblick auf die Gründung einer beruflichen »Existenz« als Selbständige beraten. Das Kompetenzzentrum Kultur- und Kreativwirtschaft des Rationalisierungs- und Innovationszentrums der Deutschen Wirtschaft stellt hier nur einen von vielen (und international weitestgehend äquivalent verlaufenden) Versuchen dar, durch die Förderung eines stärker wettbewerbsorientierten, individuellen Risikomanagements die Herausbildung einer unternehmerischen Persönlichkeit $\mathrm{zu}$ bewirken ${ }^{11}$ und "Kreative« von der Inanspruchnahme sozialstaatlicher Transferleistungen fernzuhalten. ${ }^{12}$ Im Zuge der seit 2005 regelmäßig

10 Quelle: Department for Culute, Media and Sport - Creative Industries Mapping Document, zu erst veröffentlicht am 9 April 2001. In der Verwendung der Quelle beziehe ich mich auf die Argumentation von Manske (2013: 266). Die von ihr verwendete Zitation weicht jedoch von der Zitation der hier verwendeten Originalquelle ab. Bei Manske heißt es »those activities which...«). https://www.gov.uk/government/uploads/system/uploads/attachment_data/file/1 83544/2001part1-foreword2001.pdf (zuletzt aufgerufen am 01.11.2016).

11 Ein eindrückliches Beispiel stellen etwa die in regelmäßigen Abständen initiierten Wettbewerbe des Zentrum dar, durch welche die für die kapitalistische Produktion in einzigartiger Weise typische Organisationsform der Konkurrenz eingeübt und kreatives Schaffen mit den Innovationsprinzipien der freien Marktwirtschaft in Beziehung gebracht wird. Als Preis winken den Gewinner*innen hier u.a. persönliche Screenings oder die kostenlose Teilnahme an Workshops zu Themen wie Akquise, Marketing und Unternehmensführung (Manske 2013: $272 f)$.

12 Die gesellschaftspolitische Neubewertung der vormals als schutzbedürftig gehandelten Künstler*innen zeigt sich auch in den mehrmaligen Versuchen die Künster-Sozial-Kasse ab- 
veranstalteten Jahrestagungen des Kompetenzzentrums Kultur- und Kreativwirtschaft werden die "Kreativen« nach dem Vorbild anderer westlicher Kreativindustrien dann endgültig als wirtschaftliche Modernisierer*innen in die Pflicht genommen. Während etwa der deutsche Kulturstaatsminister »Kreative« als »Innovationstreiber« stilisiert, weist der Staatssekretär des Wirtschaftsministeriums die im Kunst- und Kulturbereich vorherrschenden Lebensstile als erstrebenswerte Zukunftsmodelle aus, womit er in bezeichnendem Maße über die bisher geltende Begrenzung der wirtschaftspolitischen Zuständigkeit auf künstlerische Erwerbsformen hinausgeht und die hier vorherrschende Indifferenz zwischen Leben und Beruf zum Leitbild erklärt. Auch der Staatssekretär des Wirtschaftsministeriums hebt hier den Kunst- und Kulturbereich als gegenwärtigen Platzhalter auf dem Weg in eine kreative Wissensökonomie hervor und skizziert die Bereiche nicht minder euphorisch als volkswirtschaftlichen Hoffnungsträger (Manske 2013: 260, 270).

Staatlich forcierte Kampagnen wie diese verdeutlichen dabei sehr anschaulich die doppelte Bezugnahme von Regierungsprogrammen auf die Bevölkerung, deren Ziel und Instrument sie gleichermaßen bildet (Foucault 2004[1977-1978]: 158). Im gemeinsamen Frame der Kreativität bewegen sich so die Figuren der Kulturschaffenden und der Unternehmerin diskursiv aufeinander zu. Wohingegen Künstler*innen im Diskurs tendenziell als defizitäre Unternehmer*innen mit zu bemängelnder Marktorientierung adressiert werden, wird von Unternehmer*innen umgekehrt ein Maß an Opferbereitsschaft, Flexibilität und Unkonventionalität verlangt, das bisweilen eher mit dem Bild der intrinsisch motivierten Künstlerin assoziiert wurde.

Neben staatlichen Agenturen stellt allerdings nicht zuletzt auch die ökonomisierte Peripherie universitärer Forschung elementare Wissensquellen für die Analyse und Initiierung eines arbeitsklimatischen Wandels westlicher Nationalökonomien bereit, zu denen auch Richard Floridas geografisch-urbanistisch unterfütterten Analysen von »ethnischen « und künstlerischen (Sub)kulturen westlicher Metropolen als Ressource für ein Florieren ansässiger Kreativ-Branchen zu zählen sind. In der Tradition moderner Unternehmens- und Regierungsberatung stützen sich auch Floridas Thesen auf ein breit aufgestelltes Ensemble demografischer und fiskalischer Statistiken:

»The Creative Class Croup, founded by world renowned urbanist Richard Florida, is a global advisory firm composed of expert researchers, academics, and busi- 
ness strategists. Our proprietary data and research, gives companies and regions leading insights to achieve growth and prosperity. "13 $^{13}$

Gemäß der allgemeinen Prinzipien die Foucault mit Blick auf die Geschichte der Gouvernementalität identifiziert hat, erscheint auch die Bevölkerung moderner Postindustrienationen als "Subjekt von Bedürfnissen und Bestrebungen« sowie »als Objekt in den Händen der Regierung« (Foucault 2004[1977-1978]: 158). Wohingegen ihre komplexen und diversen Eigenheiten und Bedürfnisse im disziplinarischen Wertekorsett der rigiden Angestelltenkultur als Störfaktoren eingeschränkt werden sollten, gelten sie nun allerdings als wertvoller Nährboden um Kreativbranchen wachsen $\mathrm{zu}$ lassen.

Floridas 2002 erschienene Thesen fügen dem 2001 »Creative Industries Mapping Document« bzw. den Leitmotiven des New-Labour-Diskurses dabei auffällig wenig hinzu, sorgen aber für eine sehr viel breitere gesellschaftliche Rezeption der wirtschaftspolitischen Neudefinition künstlerisch-kreativer Tätigkeitsfelder und befördern allgemein die unternehmerische Apostrophierung von Künstler*innen und »Kreativen«bzw. ihre Bedeutung als Ressource zur Förderung allgemeiner wirtschaftlicher Prosperität in westlichen Großstädten (Manske 2013: 260, 266).

Hierzu zählen liberale Gesetzesreformen zugunsten der freien Ausübung gleichgeschlechtlicher Beziehungen ebenso, wie die zur Verfügungstellung von günstigem Wohn- und Arbeitsraum, finanzielle Förderungen und vieles mehr. Wohingegen Foucault für das 19te Jahrhundert allerdings konstatiert, dass sich die Bevölkerung gegenüber der Regierung bewusst ist, was sie will, gleichermaßen aber nichts davon weiß, "was man sie tun lässt« (Foucault 2004[1977-1978]: 158), erscheint es mit Blick auf Kreativsubjekt und progressive Technologiekultur vielversprechend, sich dieser Frage erneut zuzuwenden. So finden sich etwa unter den zahlreichen »Bürgerforen« und Anhörungen der »Kreativen« durch Stadtplanung und Kommunalpolitik durchaus sehr elaborierte Vorschläge für die Struktur-, Markt- und Humankapitalentwicklung in der Kreativwirtschaft. Hier scheint es vielmehr so, als hätten zunehmend mehr Individuen ein sehr genaues Verständnis davon, "was man sie tun lässt«, indem sie konkrete Empfehlungen darüber abgeben, wie sie regiert werden sollten, um den Regierungszielen entsprechen $\mathrm{zu}$ können. Ein besonders eindrückliches Beispiel stellt das »Memphis Creative Class Manifesto« dar, das gewissermaßen unter der Ägide Richard Floridas durch eine Gruppe namens »Creative 100« im Zuge des »Memphis Manifesto Summit « vom 30ten April bis zum zweiten Mai 2003 durch Kreative aus 48 Städten in den USA, Kanada und Puerto Rico verabschiedet wurde. ${ }^{14}$ Mit dem Gipfel richtet sich

13 Quelle: www.creativeclass.com (Webpage von Richard Florida die u.a. mit dem Claim »Data driven ideas to reach the creative class « wirbt. Zuletzt aufgerufen am 13.07.2016).

14 Die Tatsache, dass dieser Gipfel über den Tag der Arbeit hinweg abgehalten wurde ist sicher ebenso wenig zufällig, wie die pathetisch wirkenden Anleihen an die marxistische Termino- 
die selbsternannte »Creative Class« mit einem Programm aus zehn Prinzipien zur Kultivierung eines kreativen Klimas an die Lokalverwaltungen potentiell jeder Nord-Amerikanischen Gemeinde: ${ }^{15}$

»The Creative 100 are dedicated to helping communities realize the full potential of creative ideas by encouraging these principles. ${ }^{16}$

Schon an der Formulierung und Schwerpunktsetzung des ersten Programmpunktes lässt sich dabei ablesen wie im Selbstverständnis der Autor*innen die Entfaltung heterogener Lebensstile mit einer spezifischen Form von Wirtschaftlichkeitsdenken zusammenfällt. Kreativität erscheint hier zuallererst als ein Modus, in dem distinkte Aspekte des städtischen Lebens auf ihre Eignung zur Wertabschöpfung untersucht werden und die scheinbar aleatorischen Ereignisse individueller Lebenskonzepte in eine langläufige Werterzeugungskette eingegliedert werden können:

»1. Cultivate and reward creativity. Everyone is part of the value chain of creativity. ${ }^{17}$

Die Förderung von Naherholungs-, Kulturkonsumtionsmöglichkeiten, geschlechtlicher Diversität, günstiger Nutzungsräume bis hin zu esoterischen oder religiösen Angeboten sind damit nicht mehr als kulturelle Förderung sondern vielmehr als Investitionen in ein Ökosystem der Kreativität zu verstehen, das in auffälligem Maße vor allem jene Aktivitäten umfasst, die in den Kategorien klassischer Erwerbsverhältnisse gesprochen vormals dem Freizeitsegment zuzurechnen waren:

»2. Invest in the creative ecosystem. The creative ecosystem can include arts and culture, nightlife, the music scene, restaurants, artists and designers, innovators, entrepreneurs, affordable spaces, lively neighborhoods, spirituality, education, density, public spaces and third places [...] 7. Invest in and build on quality of place.

logie. Kein wunder also, dass sich hierduch auch diskursive Cegenbewegungen zur Verabschiedung eines eigenen Manifestes berufen sahen. So beginnt das Hamburger »Manifest against the creative class « mit den Worten: »Richard Florida's Chost roams throughout Europe these days. We live in a world of global cities that are involved in an interurban competition to attract investors an the so-called international knowledge worker. Keynsian economic policy has made a shift to an entrepreneurial and marginal approach to metropolitan governance. The unevitable rise of city branding and Florida's creative class theory are direct derivates from these developments. In Hamburg, artists who are designated to be the flag carriers of creativity, decided to speak up and no longer tolerate the neoliberal policy shift." Quelle: popupcity.net/hamburgs-manifest-against-the-creative-class/

»6. Every community can be the right community.«Quelle: www.creativeclass.com/rfcgdb/ar ti-cles/mani-festo.pdf (zuletzt aufgerufen am 13.07.2016).

Ebd.

Ebd. 
While inherited features such as climate, natural resources and population are important, other critical features such as arts and culture, open and green spaces, vibrant downtowns, and centers of learning can be built and strengthened. This will make communities more competitive than ever because it will create more opportunities than ever for ideas to have an impact. ${ }^{18}$

Diese Argumentation betont dabei in geradezu typischer Weise individuelle Freiheitsgrade, wobei die freizusetzenden Individuen sich und ihre Fähigkeiten gleichzeitig als Human-Ressource für den globalen Wettbewerb der Kreativstandorte einplanen:

»10. [...] The highest quality lifelong education is critical to developing and retaining creative individuals as a resource for communities. $\ll^{19}$

Das Beispiel kann als mustergültig für die Maxime verstanden werden, der nach Kreativität einerseits freigesetzt werden-, und andererseits reglementiert werden soll, um sie gezielter auf die Lösung bestimmter Probleme zu richten und von anderen fernzuhalten (Bröckling 2007: 153). »Es ist dieses Doubleblind eines konformistischen Nonkonformismus, das die Dynamik der Distinktion am Laufen hält und Kreativität zugleich entfesselt und normalisiert «(Bröckling 2014: 1). Derartige Versuche die Bedingungen zu formulieren, unter denen die Emergenz des Innovativen und Neuen kontrollierbar und vermarktbar wird, gehören inzwischen zum Standardrepertoire der Standortanalysen im allgemeinen Wetteifern von Städten, Kommunen oder Ländern um eine vorrangige Positionierung in der Wissensgesellschaft und sind insofern nichts Neues. Sie begleiten die verschiedenen Facetten der unternehmerisch organisierten Kreativindustrie allein schon deshalb, da sich ihr Primärprodukt - die innovative Idee - nicht auf direktem Weg erzwingen lässt, sondern angenommen wird, dass sich Kreativitätspotenziale lediglich auf verschlungenen Sekundärwegen im Rahmen einer komplexen Sozioökonomie steuern und gleichermaßen in eine marktfähige Richtung lenken lassen. Innovative Schaffensakte und die dazugehörigen »unternehmerische[n] Selbste fabriziert man entsprechend nicht mit den Strategien des Überwachens und Strafens, sondern indem man die Selbststeuerungspotentiale aktiviert « (Bröckling 2007: 61). ${ }^{20}$

Sehr viel interessanter erscheint demgegenüber, dass das Subjekt des kreativen Unternehmens sich erstens selbst zu Wort meldet und die Komunalverwaltungen dabei in einer Weise anspricht, die den fordernden aber auch animierenden und fördernden Parolen, die ihnen seitens der verschiedenen arbeits- und sozialpolitischen Agenturen entgegengebracht werden, in nichts nachstehen: »5. Convert a

18 Ebd.

19 Ebd.

20 Bröckling bezieht sich hier auf Nikolas Rose (2000). 
"no« climate into a "yes« climate. [...] 9. Improvise. Make things happen. Development is a »do it yourself« enterprise. ${ }^{21}$ An Beispielen wie diesen zeigt sich nicht zuletzt, dass sich eine Beschreibung von Kreativitätsimperativen und der nahezu allgegenwärtigen Kultur des Unternehmertums nicht einfach auf die Analyse von Top-Down-Regierungsprogrammen stützen kann, ohne dabei aus den Augen zu verlieren, dass sich auf diskursiver Ebene ein bestimmtes Verständnis von Selbst und Gesellschaft manifestiert, das sich in den Äußerungen und Praktiken der Individuen selbst reproduziert. Die Bevölkerung tritt hier nicht nur als steuerbare und formbare Zielprojektion von politischen Interventionen zutage, sondern vielmehr als das, was Foucault in den Schriften zur Gouvernementalität der Moderne "Öffentlichkeit« nennt (vgl. Foucault 2004[1977-1978]: 115). Die allgegenwärtige Forderung nach unternehmerischem Handeln, und eigenverantwortlicher Lebensgestaltung führt so nicht nur notwendiger Weise dazu, dass das unternehmerische Subjekt bemüht ist, die sozialstrukturellen, sozioökonomischen, psychischen und infrastrukturellen Bedingungen für sein wirtschaftliches Vorankommen und Überleben zu identifizieren, sondern die fordernden und fördernden Agenturen selbst in der durch sie vertretenen Logik auf die Herstellung der dafür notwendigen Bedingungen verpflichtet.

Der Ausdruck Kreativwirtschaft stellt zudem bereits das Ergebnis des Versuchs dar den Kreativitätsbegriff rechtlich zu formalisieren. Um aus den verstreuten Kreativbereichen über einen kleinsten gemeinsamen Nenner die Definition eines übergeordneten Wirtschaftsbereichs $\mathrm{zu}$ ermöglichen und eine Entscheidungsgrundlage dafür zu bieten, welchem Unternehmen, welchen Einzelpersonen usw. Förderungen zugesprochen und welchen sie verweigert werden, fokussiert der rechtsökonomische Diskurs in Deutschland auf einen Produktionsbezogenen Kreativitätsbegriff, der nahe an jener künstlerisch-handwerklich abgeleiteten Semantik der Schöpfung liegt, die im deutschen Sprachraum eigentlich in der Nachkriegszeit durch den utilitaristischen Kreativitätsbegriff der US-Amerikanischen Psychologie abgelöst wurde. Anders als dieser utilitaristische Begriff der Kreativität, der zunächst durch die kognitions- und sozialpsychologische Kreativitätsforschung mehr oder minder gleichbedeutend mit der Fähigkeit zu problemlösendem Handeln verwendet wurde und im Dienste der Produktivitätssteigerung zu einer Egalisierung von Kreativitätspotentialen und seiner Verstreuung über die Kurven der gaußschen Normalverteilung beigetragen hat (Bröckling 2007: 159ff), ${ }^{22}$ stellt ein solcher zum Lebenskonzept verallgemeinerter Begriff der Kreativität wirtschaftspolitische Interventionen vor ein Differenzierungsproblem. Entsprechend verengen die Wirtschaftsakteure den Kreativitätsbegriff auch überall dort, wo es

21 Ebd. [Hervorhebungen im Original]).

22 Dieser Begriff grenzt sich dabei explizit ab von einer bis dato vorherrschenden Cenie-Begriffs der Erbforschung Galtons. 
um Steuereinnahmen und -ausgaben geht, auf seine schöpferische Dimension, um konkreter Elemente habhaft werden zu können, die sich in ihrer Gesamtheit $\mathrm{zu}$ einem Bereich der Kreativwirtschaft zusammenfassen, fördern und damit ertragsfähig machen lassen.

»Über den Begriff der Kreativwirtschaft gab es in der Vergangenheit ein vielfältiges Meinungsbild. Mittlerweile wurde in Deutschland eine einheitliche Branchenabgrenzung vereinbart. Die Wirtschaftsministerkonferenz der Länder sowie die Enquetekommission des Deutschen Bundestages »Kultur in Deutschland «haben grundsätzliche Vorschläge zur Definition des Wirtschaftsfeldes vorgelegt, die eine anerkannte, klare Beschreibung des Feldes ermöglichen. Die vorgenommene Abgrenzung ist zudem mit dem Modell der EU-Kommission und mit dem weltweiten Referenzmodell, dem britischen Creative Industries Konzept, kompatibel. [...] Die Verknüpfung der Teilmärkte wird durch die Einführung des Begriffs »schöpferischer Akt «als Kern des Branchenkomplexes zusammengefasst. « ${ }^{23}$

Im Zuge von Stadt- und regionaler Wirtschaftsentwicklung werden innerhalb eines gesellschaftsweit als ubiquitär anzusehenden Klimas der Kreativität damit gezielt einzelne Segmente punktiert und durch europäische Standards geformt. Mit dem Versuch Kreativität als Kreativwirtschaft oder Creative Industries lokal zu konzeptionalisieren (d.h. innerhalb der Zugriffsmöglichkeiten auf die durch Kreativität erzeugten Produkte und die Bedingungen ihrer Erzeugung) wird der Begriff dadurch strukturanalytisch perspektiviert, wobei die in den allgegenwärtigen Kreativitäsanrufungen enthaltenen Psychologisierungen und Anthropologisierungen in den Hintergrund treten. Ein Kreativitätsverständnis, das auf die Schöpfung neuer und einzigartiger Produkte zielt, gewichtet städtische Infrastrukturen und soziale, räumlich lokalisierte Netzwerke als Entwicklungsbedingungen für kreative Potentiale entsprechend stärker als den intrinsisch-motivationalen Kern allgegenwärtiger Kreativitätsanrufungen. Kreativität erscheint in diesem Zusammenhang dann gleichzeitig "als eine ökonomische Ressource, die der Markt mobilisiert und verbraucht, wie auch als Quelle künstlerischer Produktivität« (Bröckling 2014: 2).

Lokale Kontextsteuerung entscheidet damit letztlich auch mit darüber, in welche Produktart die kreativen Potentiale überführt werden können und welche wirtschaftlichen Teilsparten bzw. »traditioneller Branchen« sich durch die Verschränkung mit angesiedelten Kreativmilieus positiv beeinflussen lassen. Neben Kunst-

23 Entsprechend werden »[u]nter Kreativwirtschaft [...] diejenigen Kultur- und Kreativunternehmen erfasst, welche überwiegend erwerbswirtschaftlich orientiert sind und sich mit der Schaffung, Produktion, Verteilung und/oder medialen Verbreitung von kulturellen/kreativen Gütern und Dienstleistungen befassen. «Quelle: Zweite Seite der »Senatsdrucksache Kreativagentur: Mitteilung des Senats an die Bürgerschaft. Aufbau des Kreativwirtschaftsclusters Hamburg. Drucksache 19/3442« vom 30.06.2009. 
und Musik ist hierbei insbesondere auch an Software- und die Entwicklung technischer Geräte zu denken: ${ }^{24}$

»Die häufig projektabhängige, vernetze Form der Arbeit in der Kreativwirtschaft greift zunehmend auf andere Wirtschaftsbereiche über und befördert damit zunehmend den Strukturwandel traditioneller Branchen. Insbesondere der Einsatz neuer digitaler Technologien ist Wachstums- und Innovationstreiber. Die Unternehmen der Kreativwirtschaft sind dabei nicht nur passive Anwender digitaler Werkzeuge, sondern geben den Technologieherstellern und -entwicklern immer wieder Anstöße für deren Weiterentwicklung. ${ }^{25}$

Auch in der Selbstreflexion kreativ arbeitender Entrepreneur*innen aus den technischen Innovationsberufen finden diese Theorien ihre Entsprechung und treten in ein konfrontatives Verhältnis zu den Utopien einer vollständig delokalisierten und enträumlichten Sozialwelt, die nicht zuletzt von den Mitbegründern der Quantified-Self-Community befeuert wurden. Wohingegen Kevin Kelly vor beinahe 20 Jahren über das Differenzkonzept »Ort vs. Raum« mit einer für die 1990er Jahre nicht ganz untypischen Internet-Euphorisierung ein kontinuierliches Verschwinden der Bedeutung von physikalischen Präsenzen als Faktor für die Entwicklung von Sozialleben und wirtschaftlichen Feldern prognostizierte, ${ }^{26}$ betont die »Kreative Klasse« 2008 im Einklang mit stadtsoziologischen Expertisen explizit die räumliche Nähe als zentrale Kategorie für das Gedeihen von Kreativprodukten (insbesondere in der Technologieentwicklung):

»Während ökonomische und kulturelle Clobalisierungsprozesse dazu beitragen, dass es »Enträumlichungen«, ein dis-embedding aus räumlichen Bindungen gibt, gibt es lokale Prozesse, die hier im Sinne der Bewahrung des cultural heritage nicht nur gegenhalten, sondern über lokale Identitätsstiftung neue Bindungsqualitäten entwickeln. Das wird traditionell einerseits (immer noch) über die Nachbarschaft von technikbasierten Unternehmen und naturwissenschaftlich-techni-

24 Für die Ermessung von Kreativität als Resource und die Rückführung dieses Wissens in den Diskurs werden sich dabei dann der üblichen gouvernementalen Mittel bedient - von denen hier vor allem die Umsatzsteuerstatistik, das Monitoring neuer Firmengründungen oder die Zählung der sozialversicherungspflichtig beschäftigten hervorzuheben ist.

25 Quelle: Zweite Seite der "Senatsdrucksache Kreativagentur: Mitteilung des Senats an die Bürgerschaft. Aufbau des Kreativwirtschaftsclusters Hamburg. Drucksache 19/3442« vom 30.06.2009.

26 Vgl. Kelly, Kevin (1998): New Rules for New Economy-Ten radical Strategies for the connected world. New York. Viking. S. 94ff: »However, the new economy operates in a »space« rather than a place, and over time more and more economic transactions will migrate to this new space." 
schen Forschungsinstituten gesehen, andererseits über die Herausbildung von Orten, die für die »innovativen Milieus « ein »seedbed « bilden. ${ }^{27}$

Dieser Diagnose schließen sich auch die Expertisen der deutschen Stadtplanung und Urban Economics an und konstatieren entwarnend den durch Kelly und andere Techno-Utopisten vorausgesagten »Kampf gegen die völlige Auflösung der räumlichen Ballung von wirtschaftlichen und sozialen Aktivitäten [...] offensichtlich erstmal gewonnen « zu haben. Wobei die verwendete Metaphorik sehr deutlich macht, dass der regionalökonomischen Beratung keine Zukunft vorstellbar erscheint, die beide Modelle vereint. ${ }^{28}$ Auch ihre Statistiken fügen sich durch die Bestätigung der Wichtigkeit von räumlicher Nähe für eine lebendige Kreativwirtschaft (z.B. über die partielle Beteiligung von »Künstlern an einzelnen Projekten« in Kreativunternehmen) in das Standortplädoyer der Regionalökonomie ein. ${ }^{29}$

Anders als Kelly betrachtet auch Florida lokale Strukturen als wichtiger werdenden Faktor für ein pulsierendes Wirtschaftsleben und bedient den Diskurs in der Tradition der Bevölkerungspolitik mit validierenden Beschreibungen zählbarer Elemente sowie der Auswertung von »zahllosen Interviews« zum Thema:

»From the countless interviews, the focus groups l've observed, and the statistical research l've done, it is apparent that place and community are more critical factors than ever before. And it appears that place, rather than beeing an abstract space as Kelly suggests, is essential to economic life. The economy itself increasingly takes form around real concentrations of people in real places [Hervorhebung im Original]. $\ll^{30}$

Unter diesen »realen Orten« würden besonders jene Metropolen ein besonders starkes wirtschaftliches Wachstum aufweisen, die durch einen besonders hohen

27 Aus dem Eintrag: »Raum als Ressource«, des Blogs »Contemporary Labour« auf dem sich vor allem im Jahr 2008 Ankündigungen und Auswertungen von Diskussionsveranstaltungen rund um die »neue Wirklichkeit des Arbeitens in Kunst, Kultur und Kreativberufen« im Raum Wien finden, in dem sich zu dieser zeit viele Medien- und Softwarefirmen anseidelten. Quelle: https://contemporarylabour.wordpress.com

28 Aus der Studie »Kreativwirtschaft in Berlin: Standorte und Standortverhalten der Unternehmen«im Auftrag des Instituts für wertorientierte Unternehmensführung (Herkommer et al. 2010).

29 Aus der Studie: „Creative Class in Berlin - Studie über Branchenstrukturen und Standortverhalten der Berliner Kreativwirtschaft« im Auftrag des Instituts für Stadt- und Regionalplanung der TU Berlin sowie ORCO Germany und Berlin Partner. Auch diese Studie bezieht sich in ähnlicher Weise auf Kelly (2008: 2).

30 (Florida 2003: 23). Oder in einer größtenteils wortgetreuen Online-Publikation: www.crea tiveclass.com-/rfcgdb/articles/4\%20Cities\%20and\%20the\%20Creative\%20Class.pdf (S. 4 des pdf). 
Grad an kultureller Diversität geprägt sind. ${ }^{31}$ Städte, in denen sich die opportunen Bedingungen für die Ansiedlung von Künstler*innen anderweitig Kreativarbeitenden, Homosexuellen oder verschiedenen weiteren Subkulturen fänden, würden demnach sehr viel schneller eine florierenden Kreativwirtschaft ausbilden, was in direkter Verbindung zu der Entwicklung der Technologie- und Mediensektoren stünde.

»The CDI [Composite Diversity Index] added together three diversity measures the Gay Index, the Melting Pot Index (a measure of the concentration of immigrants), and the Bohemian Index. The CDI providedd powerful support for the basic notion that diversity and creativity work together to power innovation and economic growth. Five of the top ten regions on the CDI were also top-ten highttech regions: San Francisco, Bosten, Seattle [...]. «2 $^{32}$

In einem regionalwirtschaftlichen Strategieentwurf bescheinigt die Planungskommission der in Floridas Rankings durchweg Spitzenplätze belegenden Stadt San Francisco ihrer ansässigen Kreativwirtschaft neben wenigen weiteren Sektoren einen etablierten Cluster darzustellen, dessen regionales Konzentrationsniveau $70 \%$ über dem US-Amerikanischen Durchschnitt läge. ${ }^{33} \mathrm{Zu}$ der Sparte der Kreativwirtschaft zählt die Skizze vor allem »technology companies, education, architechture, advertising, design firms, and traditional media.« Wobei die informationstechnologischen Branchen des Clusters die ertragreichsten Komponenten ausmachen

Die starke Polarisierung in der Verhandlung von Ort und Raum hat in der Rezeption vieler Theorien zum Thema mitunter auch starke Vereinfachungen und Missverständnisse produziert, die sich letztlich in einer ebenso starken Polarisierung zwischen »Virtualität« und »Realität« widerspiegelten. Wie Howard Rheingold in einer Anmerkung zu seiner 1993 erschienenen Publikation »The Virtual Community « selbst bemerkt ist dies nicht zuletzt auch strukturellen Zwängen zur Vereinfachung geschuldet, die der publizistische Betrieb selbst mitbringt: »When you think of a title for a book, you are forced to think of something short and evocative, like, well, »The Virtual Community«, even though a more accurate title might be: »People who use computers to communicate, form friendships that sometimes form the basis of communities, but you have to be careful to not mistake the tool for the task and think that just writing words on a screen is the same thing as real community « (1993).

32 (Florida 2012: 246). In der Neuauflage der Studie verwendeten Florida et al. einen anderen Index zur statistischen Überprüfung des Zusammenhangs zwischen »Ethnizität« und dem Wachstum in der Technologiebrachne (»Tolerance-Index« anstelle des »Melting Pot Indexes«). Nach einer erneuten Überprüfung unter diesen, nun spezifizierteren Bedingungen, liest sich hier keine positive Korrelation mehr herstellen.

33 Quelle: http://commissions.sfplanning.org/cpcpackets/DRAFT_ECOMONIC\%20STRATECY\%2 02\%2010.pdf 
würden, die auch während der ersten Krisenjahre ein kontinuierliches Wachstum ausgewiesen hätten. ${ }^{34}$

Nicht umsonst wird diese Region weltweit als Ursprungsort der New Economy betrachtet, die in den beginnenden 1990er Jahren die ersten internetbasierten Technologie-Start-Ups des Dot-Com-Booms (Manske 2006: 159) bzw. viele Multimedia-Agenturen hervorbrachte und seither als Vorbild ähnlicher planerischer Bemühungen weltweit gelten muss. Die in San Francisco ansässige Stanford University rühmt sich damit, Ikonen wie die Google-Gründer Sergej Brin und Larry Page, David Filo und Jerry Yang von Yahoo! sowie Marcian Hoff unter ihren Studierenden gehabt zu haben, der (in der meist ähnlich eng auf einzelne Biografien fokussierenden Geschichte der Hardware-Entwicklung) als Erfinder des Mikroprozessors gilt. Bis heute hat sich der ehemalige Standford Industrial Park zu dem vielleicht weltweit bedeutensten Hochtechnologiestandort entwickelt, der u.a. Apple, Intel, Google, Microsoft, Cisco Systems, Dell, Facebook, Twitter, Oracle, Amazon und Adobe beheimatete. Das Silicon Valley gilt dabei nicht nur als globale Metropole der Computertechnologieentwicklung, sondern auch als ideologisches Zentrum kreativer Querdenker*innen und marktradikaler Entrepreneure. Gleichzeitig brachte die Region allerdings auch viele progressive und radikale (Gegen)kulturen wie die Hippie-Bewegung oder die Beatniks hervor, denen in den öffentlichen Erzählungen ebenfalls unkonventionelle aber meist kreative Veranlagungen nachgesagt werden. Schon Ende der 1960er Jahre entstand in San Francisco das Schwulen- und Lesbenviertel "The Castro« und die in der San Francisco Bay Area gelegenen Städte Berkeley und Oakland gelten als die Geburtsorte des Free Speech Movement und der Black Panther Party.

Von vielen Seiten, etwa durch das sehr populäre Essay von Richard Barbrook und Andy Cameron, wird daher schon in den 1990er Jahren eine spezifisch kalifornische Ideologie konstatiert, die sich als ein »mix of cybernetics, free market economics, and counter-culture libertarianism « darstellt und ihre Antriebsenergie aus dem wechselseitigen Verhältnis sich wiederholender technologiegestüzter Befreiungsschläge sowie ihrer monetären Einhegung gewinnt: »[...] The Californian Ideology, therefore, simultaneously reflects the disciplines of market economics and the freedoms of hippie artisanship« (Barbrook und Cameron 1995).

Wohingegen Barbrook und Cameron in den 1960er Jahren noch die Gefahr darin sehen, dass die Gegenkulturen durch eine sich libertär und progressiv gebende

34 »Within the creative industries cluster, information technology services is both the largest industry and its most successful major component. Over the last business cycle, covering the recession, the industry grew over $10 \%$ per year.« Quelle: http://commissions.sfplannin g.org/cpcpackets/DRAFT_ECOMONIC\%2OSTRATECY\%202\%2010.pdf (zuletzt aufgerufen am 02.12.2016). 
»kaliforinische Ideologie « verdrängt würden, ${ }^{35}$ zählen Stadtentwicklungsplaner die Angehörigen eben jener Gegenbewegungen inzwischen zu den zentralen HumanRessourcen um wirtschaftlichen Aufschwung zu initiieren.

Unabhängig davon, ob diesen politischen Prognosen, stadtplanerischen oder sozialstrukturellen Selbst- und Fremdzertifizierungen als Vorreiter im globalen Wandel der urbanen Geschäftsfelder hin zu jenen eng verzahnten Komplexen aus Kultur- und Freizeitangeboten, Dienstleistungs- und Produktionsfeldern, die inzwischen gemeinhin als Creative Industries subsummiert werden, in irgendeiner Weise Glauben zu schenken ist, erscheint die Frage nicht ungerechtfertigt, ob es ein Zufall sein kann, dass das Phänomen des Self-Tracking ausgerechnet in San Francisco das erste mal unter einem übergeordneten Label bzw. in Form vergleichsweise kontinuierlich organisierter Strukturen in Erscheinung trat. Ob also die San Francisco von allen Seiten zugeschriebene Bedeutung als wichtigster Standort der Creative Industries, als Heimat der Creative Class, als Kulminationspunkt kritischer Politik- und Subkulturbewegungen und als führende Technologiemetropole die Herausbildung einer aktiven Szene der progressiven Entwicklung, Anwendung und Vermarktung von Selbstvermessungstechnologien mitbestimmt hat. Tatsächlich sind zwei Varianten solch einer Beeinflussung naheliegend für die eine gewisse Form von Lokalität eine Rolle spielt. Beide stellen für das Verständnis der Quantified-Self-Community bzw. des globalen Phänomens der Selbstvermessung insgesamt wichtige Schlüsselkategorien dar.

Dies betrifft erstens die bereits beschriebene kreativitätsbasierte Wirtschaftsstruktur solch einer Metropole, an deren Entstehung nicht zuletzt gouvernementale Agenturen wie z.B. die universitär angesiedelten Disziplinen Urban Economics oder Urban Development aktiv beteiligt sind und die darauf ausgerichtet ist, neue Ideen in Prototypen oder Modellversuche zu übersetzen, um anschließend auf ihren Markterfolg zu spekulieren. Zweitens betrifft dies jedoch ebenfalls ökonomische und rechtliche Zwänge, die sowohl die Arbeits- als auch die Lebensbedingungen der »Kreativen Klasse« durchziehen.

Wie Florida schreibt, weisen Regionen in denen »Kreative« in wahrnehmbarem Maße ansässig sind, schon deshalb eine exponentielle Anziehungskraft auf weitere "Kreative«, verschiedenste Akteure der Kreativwirtschaft und angrenzender Branchen aus, da sie eine Umgebung erzeugen und zur Verfügung stellen, die mehr

35 »The Californian Ideology rejects notions of community and of social progress and seeks to chain humanity to the rocks of economic and technological fatalism. Once upon a time, West Coast hippies played a key role in creating our contemporary vision of social liberation. As a consequence, feminism, drug culture, gay liberation and ethnic identity have, since the 1960 s, ceased to be marginal issues. Ironically, it is now California which has become the centre of the ideology which denies the relevance of these new social subjects « (Barbrook und Cameron 1995). 
als im üblichen Maße auf einer offenen Innovations- und Unternehmenskultur basiert. Anders als der Mainstream seien sie es gewöhnt, analog zu den Leitbildern unternehmerischer Selbstständigkeit und kreativer Problemlösungskompetenz eigenständig Ressourcen zu akquirieren, Netzwerke aufzubauen und Firmen zu gründen.

»And, their status as historically marginalized groups means that artistic and gay populations tend to be high self-reliant and receptive to newcomers. They've had to build networks from scratch, mobilize resources indipendently, and create their own oganizations and firms. For all of these reasons, regions in which artists and gays have migrated and settled are more likely than others to provide an environment that is more open to innovation, entrepreneurship, and new firm formation. $\ll^{36}$

Eine andere Möglichkeit diese Lebenslage auf einen Begriff $\mathrm{zu}$ bringen, der die implizierten Freiheits- und Flexibilitätspotentiale dieser marginalisierten Gruppen darüber hinaus als Teil einer neoliberalen Idealisierung desavouiert, ist der Begriff des Prekariats.

\subsection{Die diskursive Eigendynamik des Prekarisierungsbegriffs}

So zeichnet sich die Internetbranche bereits zu Beginn der 1990er Jahre in besonderer Weise dadurch aus, dass sie sich im Schnittfeld von Technologie- und Dienstleistungsorientierung zu einem Erwerbsfeld entwickelt, das wie kaum ein zweites die zu dieser Zeit einsetzenden Auflösungstendenzen der institutionellen Verfasstheit von Arbeit widerspiegelt, die für die tayloristisch-fordistische Regulationsweise bis dahin strukturprägend war (Manske 2013: 14). Zu denken ist hier etwa an die Erwerbsform der Alleinselbsständigkeit, die mit den Begriffen der soziologischen Ungleichheitsforschung ausgedrückt »am Randgebiet von Normalarbeit siedelt und einem Regulationsmodus unterliegt, der nicht in korporatistische Strukturen eingebunden und wegen mangelnder Zertifikate wohlfahrtsstaatlich nicht eingehegt ist (Manske 2006: 28). Dementsprechend wird der Zugang zu diesem Arbeitsmarktsegment auch nicht über die typischen Wege kanalisiert, sodass sich hier viele Autodidakt*innen finden und der Quereinstieg eher die Regel denn die Ausnahme darstellt. Der Begriff »lavoro precario« scheint in Bezug auf die Thematisierung von Lebensverhältnissen und in besonderem Konnex zur Arbeit, das erste Mal in marxistischen Kreisen der oppositionellen politischen Strukturen Italiens verwendet worden zu sein, die sich in Bezug auf Antonio Gramci mit der Lage süditalienischer Arbeitsmigrant*innen in norditalienischen Industriezentren 
auseinandersetzten. Über die weitaus populäreren Theorien zu Ungleichheitsverhältnissen in globalem Maßstab, die vor allem durch Pierre Bourdieus "La misère $\mathrm{du}$ monde und Robert Castels "Les métamorphoses de la question sociale« befeuert wurden, wurden die Szenarien empirisch ausgebreitet, die Bourdieu mit dem ebenfalls viel beachteten Essay »Prekariat ist überall« Ende der 1990er Jahre zu einem konstanten Phänomen der Gegenwartsgesellschaft erklärt hat (Candeias 2008: 124f). Auch nach dem französischen Prekarisierungsforscher Patrick Cingolani zeichnet sich der theoretische Mehrwert des Prekaritätsbegriffs dadurch aus, dass mit ihm seit den 1980er Jahren über die Beschreibung von Arbeitsverhältnissen hinaus zunehmend auch soziale Verhältnisse in ihrer Beziehung zu verschiedenen Arbeitsformen thematisiert werden. Nach und nach ist so aus einem Adjektiv, das zur Beschreibung verschiedener Arbeitsformen diente, das Subjektiv des Prekären geworden, das die Soziallage einer ganzen Gruppe definiert bzw. sie unter diesem Begriff als solche konstituiert (Cingolani 2013: 33).

»Dem etwas angestaubten Begriff »creative industries« haben sich neue wie »digital bohemia« oder Prekariat hinzugesellt. Und während Feuilleton und Politik noch über Begrifflichkeiten diskutieren, haben sich schon lange neue Praxen des Arbeitens, Geldmachens und Zusammenwirkens entwickelt. [...] Das Leben und Arbeiten der Kreativen Klasse verläuft auf dem schmalen Grat zwischen Kunst und Kultur, Job und Hobby und zwischen Freizeit und Prekariat. Die Übergänge sind fließend und nicht immer klar zu trennen. Aus diesem Grund haben sich Künstler, Kulturschaffende, Studierende und EventorganisatorInnen zusammengefunden, gemeinsam die Bedürfnisse der Kreativen Klasse zu ergründen, deren kulturelles Selbstverständnis zu manifestieren und die Erfordernisse für das Leben als kreativ Arbeitende in einer Stadt aufzuzeigen. ${ }^{37}$

An einer derartigen Definition orientiert sich auch die öffentliche Debatte im deutschen Sprachraum, die sich vor allem an einer Studie der Friedrich-Eberst-Stiftung aus dem Jahr 2006 entzündete, durch die etwa $8 \%$ der arbeitsfähigen Menschen aus den alten und neuen Bundesländern zu einem Prekariat der Abgehängten erklärt wurden, mit dem sich für die Sozialstrukturanalyse zunächst das Bild eines »stark ostdeutsch und männlich dominierte[n] « Typs von "Arbeitslosen « verband, der in besonderem Maße »von sozialem Ausschluss und Abstiegserfahrungen« geprägt sei. ${ }^{38}$ Diese Vorstellung, der in prekären Verhältnissen lebenden Entitäten ist im deutschsprachigen Diskurs damit lange kongruent zur französischen Ungleichheitsforschung, die (wie bei Bourdieu besonders deutlich wird) von Menschen in

37 Quelle: https://contemporarylabour.wordpress.com (zuletzt aufgerufen 08.11.2016).

38 «Gesellschaft im Reformprozess« - Die Friedrich-Ebert-Stiftung untersucht Reformbereitschaft der Deutschen«Quelle: www.fes.de/aktuell/documents/061017_Cesellschaft_im_Refo rmprozess_komplett.pdf (zuletzt aufgerufen 20.10.2016). 
einer prekären Lage annimmt, dass sie strukturell in ihrer Fähigkeit beeinträchtigt sind Zukunftsprojekte zu entwerfen, da ihnen die Voraussetzung für »jegliches sogenannte rationale Verhalten« fehle, »angefangen beim ökonomischen Kalkül« (Bourdieu 1998: 109). Ein solches Bild ist in der Prekarisierungsdebatte zur Zeit der Friedrich-Ebert-Studie allerdings längst nicht mehr unangefochten und wurde bereits vielfach mit soziologischen Studien konfrontiert, die das Phänomen des Prekariats nicht auf spezifische Milieus der »Social Underclass « oder eine besonders deprivierte Form des Subproletariats begrenzt sahen, sondern es in viel weitreichenderer Hinsicht vor allem mit den sozialkritischen Prämissen der GenderStudies und zeitdiagnostischen Analysen des arbeits- und sozialpolitischen Wandels verbanden, der sich im Zuge der 1990er Jahre zu vollziehen begann. So verbindet sich mit dem Begriff der Prekarisierung geschlechterpolitisch gewendet nicht nur ein abweichendes Regulierungsniveau des Tarifrechts, des Einkommens oder der rechtlichen Schlechterstellung anhand von Leih- und Kurzarbeitsverhältnissen, sondern z.B. auch die mangelnde Anerkennung von Hausarbeit, die seit jeher einer betrieblichen Ausgliederung noch dazu gänzlich unbezahlter gesellschaftlicher Arbeitskraft gleichkommt. Der meist industriegesellschaftlich verengte Deutungsrahmen der Prekarisierungsdebatte vernachlässigt dabei nicht nur, dass prekäre Lebensverhältnisse für Frauen (ob im Kontext von häuslicher Reproduktion oder z.B. im Erwerbsfeld der Care-Berufe) bis heute eher die Regel denn die Ausnahme darstellen (Candeias 2008: 135f), ${ }^{39}$ vielmehr bemisst sich die Sozialkritik der Ungleichheitsforschung einseitig an den Standards der fordistischen Erwerbsregime, ohne die mit ihnen verbundene geschlechterspezifische Arbeitsteilung nach Maßgabe des Familienernährermodells in Frage zu stellen. Eine derartige normative Überhöhung des androzentrischen Normalarbeitsverhältnisses ist damit aus geschlechterpolitischer Perspektive für sich genommen bereits problematisch (vgl. Manske 2007: 33). Der geschlechtliche und industriegesellschaftliche Bias des öffentlichen Prekarisierungs-Diskurses wird allerdings Mitte der NullerJahre zunehmend durch Analysen irritiert die neben dem vermeintlich männlich geprägten Milieu des betrieblichen Niedriglohnsektors, das durch die Studie der Friedrich-Ebert-Stiftung als sozial »abgehängtes Prekariat« bestimmt wurde, eine ganze Reihe an Erwerbsmodellen unter dem Prekaritätsbegriff versammeln, die zwar unsicher und sozialrechtlich kaum abgesichert sind, deren sozialstrukturelle Zusammensetzung sich im Sinne einer »Prekarisierung auf hohem Niveau (Manske 2007) allerdings eher aus den gehobenen Rängen entsprechender Schichtungsmodelle rekrutiert. Bemessen an den Bestimmungskriterien der Ungleichheitsforschung (also der Unterschreitung von Standards in Bezug auf das Normalarbeitsfortsetzt. Der verminderte Rechtsstatus von Frauen die in Care-Berufen ist hier nur als ein Beispiel zu nennen. 
verhältnis) müssen demgemäß auch Wissenschaftler*innen, Journalist"innen und Webdesigner*innen zum Prekariat gezählt werden (vgl. Candeias 2008: 12), ohne dass die deterministische Gleichsetzung des Prekariats mit einem Milieu der lethargischen Perspektivlosigkeit oder vergleichbaren Zuschreibungen auf sie anwendbar wären.

Die Frage ob die prekäre Arbeitskraftunternehmerin vor dem Hintergrund dieses strukturellen Wandels inzwischen zu einer neuen Grundform der Ware Arbeitskraft geworden ist, lässt sich aus diskurstheoretischer Perspektive allerdings schon allein aufgrund der für die sozialkonstruktivistische Epistemologie typischerweise eher unverbindlichen Verwendung des Wortes »ist « nicht klar beantworten, dass als flektierte Form des Verbs »sein« zwar die Zielmarke der soziologischen Sozialstrukturanalyse bildet, aus poststruktureller Sicht aber immer nur eine vorübergehende kollektive Übereinkunft meinen kann, deren Herstellungsbedingungen gleichsam den Hauptanalysefokus bilden. Wie Bröckling bemerkt ist daher auch der Status der diskursiven Figur »Künstlerin« oder »Unternehmerin« selbst prekär. »Ein ganz und gar unternehmerisches Selbst gibt es so wenig wie einen reinen Markt. [...] Künstler und Unternehmer ist man immer nur à venir - stets im Modus des Werdens, nie des Seins « (Bröckling 2014: 2). Auch wenn sich die Diskursforschung selbst keine Aussage über den »Ist-Zustand « der »Wirklichkeit« zu treffen erlaubt, kann sie die Ergebnisse entsprechender Forschungen aber nach eigenen Prämissen als Indikatoren dafür nehmen, unter welchen Vorzeichen gesellschaftliche Wirklichkeit konstruiert und problematisiert wird. In dieser Hinsicht scheinen die soziologischen Analysen zu den Erwerbsbiografien aus den Bereichen verschiedener Kultur-, Wissens-, Bildungs- und Medienberufe dabei vor allem die Annahme zu befördern, dass die Deformalisierung von Arbeitsstrukturen vergleichsweise häufig mit einem hohen Maß an Eigeninitiative und Experimentierbereitschaft einhergeht und von den potentiell betroffenen hier gerade nicht als deterministische Kategorie ausgewiesen wird. Insbesondere durch qualitative Untersuchungen wie z.B. Interviews mit akademisch gebildeten, kulturorientierten Anhänger*innen der Wissens- oder Medienbranchen großstädtischer Ballungsräume reproduzieren sich dabei unweigerlich Selbstbilder die im weitesten Sinne kongruent zu den identitätspolitischen Distinktionen sind, die sich in den Publikationen der Creative Class finden. Im Desiderat dieser Interviews wird Arbeit z.B. als eine Sinnquelle dargestellt und vom Gelderwerb als ausschließliche Funktion abgegrenzt (Manske 2007: 188). Die hiermit oftmals in Zusammenhang stehende prekäre Lebenslage erscheint in den methodisch kontrollierten Selbstauskünften als kollateraler Schaden an der strukturierten Lebens- und Erwerbsplanung, der aber durch ein erhöhtes Maß an Selbstbestimmung kompensiert wird. Letzteres hat etwa Manske anhand von alleinselbstständigen Webdesigner*innen exemplarisch für den Bereich dienstleistungsorientierter Technologie- und Medienberufe untersucht (Manske 2006: 165). Dabei setzt sie den vornehmlich deterministisch 
verwendeten Begriff der Prekarität in den Kontext einer liberal- und freiheits-ideologischen Abgrenzung gegenüber konformen Lohnarbeitsverhältnissen.

Ob im betrieblichen Kontext oder in der Alleinselbstständigkeit, der primäre Arbeitsmodus zeichnet sich dabei seit den späten 1990er Jahren zunehmend als eine unmittelbare Beziehung zum Markt aus. Zu den stärksten Effekten, den eine solche Internalisierung des Marktes innerhalb der Betriebe bewirkt (Mondaschl und Sauer 2000), zählt z.B. dass Zumutungen im Arbeitsprozess anders als in den hierarchisch strukturierten Arbeitsverhältnissen nicht länger als kritisierbar erscheinen, da Risiko und Verantwortung an die Arbeitenden weitergeleitet wurde (Spilker 2010: 111). Manske argumentiert in Bezug auf Alleinselbstständige ähnlich und spricht von einer radikalen Vermarktlichung. Radikal marktvermittelt ist demnach die Lebenslage, in der sich jede nicht marktkonforme Lebensentscheidung negativ auf die eigene Stellung am Markt auswirkt und sich im Umkehrschluss Veränderungen am Markt unmittelbar auf die Lebenslage auswirken kann. Der Term radikal marktvermittelt ist daher in vielen Fällen synonym mit dem Begriff prekär (Manske 2006: 165).

Ein Umstand, der sich durch die periodischen Strukturkrisen der neuen Technologiemärkte noch einmal verstärkt. Vor allem Ende der 1990er Jahre führte dies dazu, dass die sozioökonomischen Eruptionen auch die New Economy erschütterten und zumindest zeitweise ihres euphorischen Grundtenors beraubten: Der Begriff Prekariat wurde zu diesem Zeitpunkt zu einer Selbstbezeichnung.

So gewendet erscheint das Prekariat selbst nach Maßgabe der soziologischen Ungleichheitsforschung weniger als eine klar identifizierbare Erscheinung inmitten einer ansonsten konstanten und generalisierbaren Form von Arbeitsverhältnissen, sondern vielmehr als ein Teilprozess eines grundsätzlichen arbeitsgesellschaftlichen Strukturwandels, der gleichermaßen Ausdruck »einer allgemeinen gesellschaftlichen Kultur der Unsicherheit « (Candeias 2008: 126) ist, ${ }^{40}$ die in elementarem Sinne mit dem hegemonialen Imperativ der Selbstaktivierung im Zusammenhang steht. Das Phänomen der Prekarisierung ist damit fernab der Kategorisierungen der soziologischen Sozialstatistik auch im poststrukturalistischen Sinne in zweifacher Hinsicht interessant, um die Subjektverhältnisse moderner Arbeitswelten zu beschreiben: Zum einen mit Blick auf die diskursive Eigendynamik des Begriffs, der von einer distinktiven Sozialkategorie der Sozialstrukturanalyse oder statistischen Sozialwissenschaft zu einer (in weiten Teilen selbstkritischen) Identitätsbezeichnung avanciert ist, unter der sich inzwischen die Wissens- und Kreativarbeitenden in Abgrenzung zur linearen Erwerbsbiografie selbst versammeln. Zum zweiten lässt er sich allerdings auch als nicht-diskursive Seite einer postfordistischen Gouvernementalität fassen (vgl. Spilker 2010: 103), die im Zeichen von

40 Die auch Pierre Bourdieu im Sinn hatte als er in einem vielbeachteten Essay danach fragte ob das Prekariat nicht längst überall sei (1998). 
Projektarbeit, Flexibilisierung und Vermarktlichung eine strukturelle Unsicherheit errichtet, deren Ballungszentren sich mit den westlichen Technologiemetropolen überlagern. Wobei prekäre Beschäftigungsverhältnisse, wie das Ideal künstlerisch kreativer Arbeit diskursiv nicht auf die Kreativwirtschaft begrenzbar sind, sondern als Prototypen neuer Normalarbeitsverhältnisse (vgl. Candeias 2008: 123) oder zumindest zusätzlicher Normalarbeitstsstandards (Pongratz und Voß 2003: 242) in die Gesellschaft ausstrahlen.

Den sozialstrukturellen Annahmen über die allgemeine Verschärfung von Arbeitsbedingungen im Kontext der New Economy kann daher insoweit gefolgt werden, als dass sie neben den appellativen und präskriptiven Anrufungen, auf die sich die Gouvernementalitätsstudien konzentrieren, durchaus ökonomische und rechtliche Erklärungsfaktoren für die andauernde Konjunktur der mikropolitischen Ratio des unternehmerischen Selbst liefern, das in zunehmend mehr gesellschaftlichen Bereichen eine kaum mehr in Zweifel zu ziehende Plausibilität für sich beansprucht (vgl. Bröckling 2007: 50).

\subsection{Self-Tracking und Kreativwirtschaft}

Anhand der unterschiedlichen Einflüsse, Anreize und Dringlichkeiten, die im Zuge der vorangegangenen Spurensuche versammelt wurden, lässt sich so ein zumindest grobes Bild der Subjektivität nachzeichnen, für dessen Erreichen die Entwicklung und Anwendung von Selbstvermessungstechnologien als ein passabler Weg erscheint. Selbstvermessungstechnologien lassen sich vor dem Hintergrund kulturkritischer Subjekttheorien und zumindest exemplarischer Betrachtungen interdiskursiver Schnittmengen (z.B. mit den Themenfeldern postfordistischer Arbeitskultur und regionaler Wirtschaftspolitik) als situative, soziotechnische Arrangements aber auch als Design-Objekte und ästhetische Artefakte begreifen, in denen sich bestimmte Problemszenarien und distinktive Identitätsmerkmale qua Inskription bündeln. Dabei ist zum einen an die zunehmende Ablehnung von konformistischem Massenkonsum zu denken, die im Zuge voranschreitender Individualisierung die Erosion der Grenzen zwischen Konsumtion und Produktion und persönlicher Identität bedingt. Zum anderen erscheint es vielversprechend, das Phänomen der Selbstvermessung auch mit Blick auf die grundsätzliche Reinszenierung gesellschaftlicher Wertvorstellungen in Bezug auf Arbeit zu betrachten, die sich unter den Vorzeichen subjektiver Selbstverwirklichung in libidinös besetzte Idealisierungen kreativer, eigenständiger Tätigkeiten projizieren. Hierzu tragen auch die tiefgreifenden strukturellen Veränderungen in den Sozialsystemen moderner Staaten bei, die ihrerseits das Ende des Vorsorgestaats (Ewald 1993[1983]) und kollektiver Wohlstandsversprechen einläutet und sozioökonomische Unsicherheit im Zuge von »Reaganomics«, »Thatcherism« und 
»Agenda 2010« auch für hochqualifizierte Wissens- und Technologiesegmente auf Dauer stellen. Anders als das Bild, das die statistische Sozialstrukturanalyse lange von »dem Prekariat« gezeichnet hat, werden unter Prekarisierten in jüngeren soziologischen Untersuchungen, aber auch im diskursiven Austausch der Kreativarbeitenden untereinander, nicht mehr nur Angehörige eines lethargischen und perspektivlosen Subproletariats summiert, sondern ebenso die Angehörigen einer agilen Avantgarde, die analog zu den Idealen unternehmerischer Selbstständigkeit in der Lage sind, eigenständig Ressourcen zu akquirieren, Firmen zu gründen und ihren beruflichen Fokus unentwegt zu verschieben. Aspekte, die spätestens mit dem Crash des »Dot-Com-Booms « und dem Ende der »Roaring Nineties« (Manske 2007: 65) zu überlebenswichtigen Fähigkeiten in den Technik- und Medienberufen werden, da die Deformalisierung von tayloristisch-fordistisch regulierten Arbeitsstrukturen sowie ihrer staatlichen Absicherung in der Regel durch ein hohes Maß an Eigeninitiative und Experimentierbereitschaft ausgeglichen werden muss (Manske 2007).

Self-Tracking-Tools lassen sich dementsprechend sowohl als ein technologisches Derivat fassen, in dem gemäß eines zur Norm ausgewachsenen Klimas der Kreativität Ideenreichtum und elaborierte Problemlösungsfähigkeiten praktisch unter Beweis gestellt werden, als auch als ein Modus in dem intrinsisch auf Rationalitätskrisen im arbeitsstrukturellem und wohlfahrtsstaatlichem Wandel reagiert wird.

Während der sog. Digital-Labour-Conference bemerkten die Teilnehmer*innen selbst, dass das Jahr 2008, das auch das Jahr ist in dem Quantified Self das erste mal öffentlichkeitswirksam in Erscheinung trat, gleichermaßen durch eine Vielzahl technologischer Innovationen und Krisenereignisse geprägt war:

»The past decade was not only about advances in digitization, increased processing power, the popularization of cloud computing and the »sharing economy; « it was also about the crash of the financial system in 2008 , vast attacks on employment and worker rights[...]. « ${ }^{41}$

Die Projektförmigkeit oder die verschiedenen Effizienz- und Optimierungsimplikationen dieser Technologien sind dabei zugleich als ein Spiegel steigender Anforderungen in Bezug auf die individuelle erwerbswirtschaftliche Leistungsfähigkeit und als das Ergebnis produktorientierter Innovationsprozesse $\mathrm{zu}$ betrachten, im Zuge derer jede Erfindung noch augenblicklich auf ihr potentielle Markttauglichkeit geprüft wird, was die allerseits geforderte Selbsterfindung mit einschließt.

Zudem erscheint es sinnvoll Self-Tracking-Tools, trotz ihrer potentiell globalen Anschlussfähigkeit als digitale Internettechnologien, auch unter Berücksichtigung lokaler Spezifika zu betrachten, da sie mitunter innerhalb der Innovations- 
netze großstädtischer Tech-Communities oder aus künstlerisch-kreativen JointVentures hervorgehen. Schon fragmentarische Einblicke in den Diskurs der stadtplanerischen Regionalentwicklung offenbaren sehr deutlich, wie anhand entsprechender Interventionen aktiv versucht wird, städtische Umgebungen als »Seedbed « für die kreative Technologie-Entwicklung lokal zu kultivieren und ein hierzu passendes Arbeitssubjekt zu erzeugen. Unter dem Label Creative Industries laufen die lange Zeit konträr gehandhabten Stereotype der Künstlerin und der Unternehmerin dabei mehr und mehr aufeinander $\mathrm{zu}^{42} \mathrm{Im}$ Zuge dieser Gegenbewegung werden Künstler*innen im Diskurs tendenziell als defizitäre Unternehmer*innen mit zu bemängelnder Marktorientierung adressiert, während von Unternehmer*innen gleichzeitig ein Maß an Opferbereitschaft, Flexibilität und Unkonventionalität erwartet wird, das bisweilen eher als typisch für die intrinsisch motivierte Künstlerin galt. »Kreativität« erwächst auf diesem Weg daher sowohl zu einem Subjektivationsziel wirtschaftspolitischer Diskurse und nicht-diskursiver Maßnahmen, als auch zu einem Modus der Problemlösung und Selbstformung. Insbesondere das Silicon Valley wird in diesem Zusammenhang als geradezu sinnbildlich für eine entrepreneuriale Kultur betrachtet, in der die wirtschaftliche Infrastruktur aus führenden Technologiebranchen und eine strukturelle »Prekarisierung auf hohem Niveau « (Manske 2007) als materielle Bedingung auf den gesellschaftlichen Imperativ der selbstständigen Problemlösungskreativität treffen. Die hieraus erwachsende postbürokratische und vornehmlich projektförmig organisierte Arbeitskultur bindet das wirtschaftliche Überleben entsprechend an ein Selbstverständnis, das produktiv zwischen den Extrempolen kreativer Unkonventionalität und unternehmerischer Rationalität changiert und auf jedes individuelle oder gesellschaftliche Problem mit einer technologischen Innovation zu reagieren versucht. Der Umstand, dass viele Self-Tracking-Technologien in eben dieser Weise auf das rhetorisch-ideologische Rahmenwerk des »Californian way of life« (Reckwitz 2010: 555) referieren und sich das überindividuelle Selbstverständnis als "Quantified Self« ausgerechnet in den wissensintensiven Arbeits- und Konsumtionsmilieus westlicher Postindustrienationen formuliert, kann daher ebensowenig als zufällig betrachtet werden, wie ihre sozialrevolutionäre Lackierung als politische Bewegung.

\subsection{Datataining, Data Selfies, Data Artists}

Obgleich die Anrufung der Unternehmerin, an der die Künstlerin weit stärker parasitiert als umgekehrt, schlägt sich die gegenseitige Indienstnahme (Bröckling

42 »Obgleich die Anrufung des Unternehmers weitaus stärker an der des Künstlers parasitiert als umgekehrt, verändert diese gegenseitige Indienstnahme ohne Zweifel das Selbstverständnis beider« (Bröckling 2014: 1). 
2014: 15) beider Leitbilder nicht nur in Form von diskursiven Selbstverständnissen, Mustern des Begreifens von Arbeit als intrinsische Kreativakte oder nichtdiskursiven Modi der Arbeitsorganisation nieder, sondern schreibt sich auch in die Arbeitsprodukte selbst ein. Dies betrifft die Präsentationen von Vermessungsprojekten während der zahlreichen Meetups und Konferenzen, ihre kontinuierliche Dokumentation auf Blogs oder im Social Web, sowie die Entwicklung der Vermessungs- oder Visualisierungssoftware selbst. So sind während der Projektpräsentationen einfache tabellarische Datensätze oder dergleichen eher selten zu sehen. Vielmehr überbieten sich die Self-Tracker*innen unentwegt nicht nur in der Originalität der angewendeten Methoden, Korrelationen oder der Untersuchungsgegenstände, sondern auch in der Ausgefallenheit ihrer visuellen Aufbereitung. Die Daten-Collagen der Quantified-Self-Mitglieder scheinen dabei nicht nur der erleichterten Vermittelbarkeit einfacher Zählungen oder komplexer mathematischer Beziehungen durch die Verwendung von Diagrammen, Graphen und Kurven zu dienen. Vielmehr erwecken die Präsentationen der Quantified-Self-Mitglieder und auch die Grafiken der Apps proprietärer Software-Firmen zusätzlich den Eindruck, sich mal konform, mal divergent an einem ästhetischen Kanon auszurichten. Ein emotional ansprechendes Datataining, das nicht selten durch enthusiastische Bewertungen oder die sprachliche Unterstreichung der eigenen Begeisterungsfähigkeit und ein lustvolles Experimentieren mit der eigenen Verdatung begleitet wird:

»I wrote an open-source app to track everything. It draws nice charts [...] What I now have... is wonderful! I have a SQLite database on my computer and can ask questions like: how much do I sleep in average? «3

Das verallgemeinerte Dispositiv der Kreativität etabliert auch hier ein Regime des Neuen, das auf die Hervorbringung ästhetischer Reize abzielt (Bröckling 2014: 15) und so das beständige Anwachsen der ohnehin bereits variationsreichen Palette verschiedener Darbietungsformen von Selbstvermessungen garantiert. Die Annual Reports der Self-Tracker*innen Nicholas Feltron und Lillian Karabaic gelten innerhalb der Quantified-Self-Community im Hinblick auf Ausgefallenheit und ästhetisches Arrangement inzwischen nahezu als Institutionen, denen nicht selten ein stilprägender Einfluss auf andere Selbstverdatungspräsentationen nachgesagt oder auf die sich in teils ehrfurchtsvoll anmutendem Duktus berufen wird.

»I have a few data viz heros! Jer Thorpe, of the new york times, makes beautiful interactive data visualizations and is one of the best speakers I have ever seen.

43 Antwort einer Self-Trackerin auf die Frage nach ihrer Motivation. Quelle: https://forum.qua ntifiedself.com/thread-i-wrote-an-open-source-app-to-track-everything-it-draws-nice-charts (inzwischen nur noch über Wayback-Maschinen abrufbar). 
Nicholas Feltron [...] is a compulsive self-tracker who releases a gorgeous printed yearly report. ${ }^{44}$

Die grafischen Annual Reports von Feltron, der als führender Designer an der Facebook-Time-Line mitgewirkt hat, werden diskursiv dabei als Kreuzungsprodukt eines musengeküssten Genies und »mindmappenden « Querdenkers (vgl. Bröckling 2007: 154) gehandelt, dem trotz glaubhafter Zurschaustellung ästhetischen Selbstzwecks der unternehmerische Sinn nicht abhanden kommt. Feltrons Annual Reports, mit denen er seine gesammelten Kommunikations-, Gesundheits oder Bewegungsdaten eines Jahres in Form mehrfarbiger Tabellen, Kurven und Diagramme collagiert, sind mit einer limitierten Auflage von 3000 Kopien (»[p]rinted with 4 color process and additional 3 spot colors including fluorescent and metallic inks with metallic foil-stamped cover $)^{45}$ käuflich zu erwerben. Zudem arbeitete er an der App Daytum mit, die in besonderer Weise darauf ausgerichtet ist, die ästhetische Darstellung von Lifelogging-Daten zu ermöglichen.

Auch der neunte Annual Report von Lillian Karabaic, der sich u.a. auf eine Studie zu Burnout im Zusammenhang mit dem Antritt eines neuen Jobs befasst und der auf der Quantified-Self-Website auf Grund der »entertaining visualizations ${ }^{46}$ angepriesen wird, bezieht seine Inspiration gleichermaßen aus verschiedenen Darstellungsformen des Feltron Reports sowie des Economist Magazine. ${ }^{47}$

Nicht zuletzt anhand des gemessenen Zeitaufwandes für die unterschiedlichen Arbeitsschritte lässt sich über eine Gewichtung der Geltung mutmaßen, die hier jeweils auf die Erfassung von Daten und ihrer visuellen Aufbereitung entfallen:

»This report, which is the 9th annual quantified self report by Lillian Karabaic, involved 31.6 hours of design, 5.4 hours of writing, and 6.2 hours of data work. $^{48}$

Websites und Apps wie Daytum, die derart zur Setzung ästhetischer Schwerpunkte tendieren, bilden zwar Ausnahmen innerhalb des Diskurses, allerdings sind sie dennoch keine Randerscheinungen. Vielmehr polarisiert sich an ihnen die diskursive Aushandlung des Verhältnisses von Funktionalität und Ästhetik, wobei sie die entsprechenden Grenzen markieren. Wohingegen die Dokumentation eines

44 Self-Trackerin Katie McCurdy in einem Interview mit Quantified Self. Quelle: http://quantifie dself.com-/2012/03/talking-data-with-your-doc/ (zuletzt aufgerufen am 14.02.2017).

45 Quelle: http://feltron.bigcartel.com/product/2014-annual-report-pre-order (zuletzt aufgerufen am 14.02.2017).

46 Quelle: http://quantifiedself.com/?s=annual+report\&x=0\&y=0 (zuletzt abgerufen am 14.02. 2017).

47 »This report was inspired by the design of The Economist magazine«; »The average day wheels were inspired by Nicholas Feltron's annual report.«Quelle: http://anomalily.net/the -year-2016/ (zuletzt aufgerufen am 14.02.2017).

Quelle: http://anomalily.net/the-year-2016/ (zuletzt aufgerufen am 14.02.2017). 
Show\&Tell in New York City die Präsentation einiger Daytum-Daten-Collagen durch Nicholas Feltron als "Beauty of Self-Tracking « ${ }^{49}$ betitelt, bleibt eine Kritik an der deutlichen Übergewichtung von Visualisierungstechniken bei gleichzeitigem Mangel an analytischem Mehrwert in Form vieler User-Kommentare ${ }^{50}$ aber auch anderer Autoritäten der Quantified-Self-Community nicht aus:

»Daytum, which is mainly about self-expression, will be nifty for the person who uses data mainly as a feature of personal identity. Daytum's origin is in the Feltron Annual Report by Nicholas Feltron; an annual report serves many purposes, but data analysis is not one of them. ${ }^{51}$

Dennoch liegt der Grund weshalb ihnen im Self-Tracking-Diskurs eine Sonderstellung zugewiesen wird darin, das ihnen eine graduelle Überbetonung von Ästhetisierungsformen und keine kategorische Unvereinbarkeit mit den Leitmaximen der Self-Tracker*innen unterstellt wird. Extreme wie die Annual Reports von Feltron und Karabaic lassen sich letztlich als Ausdruck einer kontrollierten Unangepasstheit lesen, deren Kultivierung zu transformierbaren Werten sowohl im Bereich der Kunst als auch der Ökonomie führen kann, »weil sie authentischer Ausdruck künstlerischer Freiheit ist beziehungsweise ökonomisch gesehen ein Alleinstellungsmerkmal darstellt« (Bröckling 2014: 16). Von den Extrempolen abgesehen, fungieren überdeutliche Ästhetisierungen dieser Art auf ein bestimmtes Identitätsnarrativ des Self-Tracking-Diskurses, das sich vor allem im Bereich des Lifeloggings findet, und auf einer möglichst umfassenden, lückenlosen und langwierigen Protokollierung einzelner Lebensaspekte basiert, ohne dass dabei zwingend

49 Quelle: http://quantifiedself.com/2009/06/nyc-qs-showtell-i-the-beauty/ (zuletzt aufgerufen am 14.02.2017).

50 Ein Kommentar des Users »Michael« unter dem Artikel: »The 2009 Feltron Annual Report OCD Made Sexy « auf https://flowingdata.com: »It's definitely attractive. Unfortunately it is also seemingly pointless ... unless the point is to look pretty. There is no real value to the reader and he makes no attempts to capitalize on his findings and change his actions from year to year. He just tracks information and spits it. Again pretty, but pretty useless. «Quelle: http:// flowingdata.com/2010/01/26/the-2009-feltron-annual-report-ocd-made-sexy/ (zuletzt aufgerufen am 14.02.2017). OCD steht für »Obsessive-Compulsive Disorder «- eine im ICD-10 geführte Form von zwanghafter Persönlichkeitsstörung, die sich durch Rigidität, Perfektionismus und ein ausgeprägtes Kontrollbedürfnis auszeichnet. >Flowingdata ist die Website von Nathan Yau, der nach der Promotion in Statistik an der University of California als Programmierer und Informationsdesigner tätig ist. Er ist Autor zweier Bücher über datenvisualisierung. Über die Website bietet er Tutorials zur visuellen Aufbereitung von Daten an. »l strive to help perople understand data in both their personal and professional lives. Hopefully they have fun in the process. «Quelle: https://flowingdata.com/membership (zuletzt abgerufen am 15.02.2017).

51 Quelle: http://quantifiedself.com/2009/04/flowing-data-dream-of-a-perf/ (zuletzt aufgerufen am 14.02.2017). 
nach Korrelationen zwischen verschiedenen Variablen gesucht wird. Die DatenPraktiken, die diesen Modellierungen zu Grunde liegen, zielen in besonderem Maße auf solche Ereignisse, denen für gewöhnlich kaum Aufmerksamkeit geschenkt wird. Für viele der enthusiastischen und akribischen Lifelogging-Projekte ist der Mangel einer klaren Zieldefinition daher symptomatisch. Diese Projekte folgen nicht selten der Logik eines freischwebenden Empirismus, der sowohl Fragen als auch Antworten aus dem gleichen Datensatz generiert.

In der ästhetischen Hülle bunter Diagramme konvertieren Apps wie Daytum Tätigkeiten, die in anderen Kontexten (oder eben schon in Form anderer Datenvisualisierungen wie einfacher Tabellen) kaum einen Aufmerksamkeitswert beinhalten würden, in kreativ anmutende distinktive Formen der Selbstdarstellung: „Create the ultimate data-selfie. «Ähnlich wie Daytum reagiert auch die App Frickbits dabei in paradoxer Weise auf ein grundlegendes Problem der datenbasierten Selbstdarstellung. Frickbits, Daytum und viele andere Lifelogging-Tools, die die Darstellungsvarianz über analytische Anwendungsmöglichkeiten stellen, wirken wie experimentelle Aufwertungsversuche von eher variationsarmen Lifeloggs, deren Monotonie sich ein Stück weit schon durch die erforderte Regelmäßigkeit dieser Datenpraktik bedingt und so in den meisten Fällen zuallererst eine numerische Sichtbarkeit von Alltag erzeugt. Die akribischen Arbeiten der Konzeptualisten Tehching Hsieh oder On Kawara, der Teilaspekte seines Lebens in den 1970er Jahren durch Schwarz-Weiß-Fotos und eine Stempeluhr protokollierte, aber auch schon die Tabellen zur Volkswirtschaftlichen Gesamtrechnung des Statistischen Bundesamtes, bieten eindrückliche Gegenbeispiele für die glanzlose Tristesse nüchterner Datensätze oder protokollarischer Fotografie.

Da sich nicht nur die verdateten Tage der Lifeloggs in akkumulierter Form zu einem prototypischen Alltag normalisieren, sondern auch die Datensätze verschiedener Lifelogger*innen zumindest strukturtypische Ähnlichkeiten aufweisen, wundert es nicht, dass überall dort, wo sie zur Selbstexpression genutzt werden, die spezifische Form der Darstellung, also die individuellen Fähigkeiten der Bearbeitung und Präsentation in der Vordergrund rücken.

Vergleichbar mit den Filtern von Instagram, die jedes banale Alltagsfoto in die warme Atmosphäre karibischer Urlaubsbilder zu tauchen vermögen, stellen sich allerdings auch im Fall von Data-Selfies Gewöhnungseffekte ein, insofern der Aufmerksamkeitseffekt relational zur steigenden Nutzung der Technologien und der Verbreitung ihrer medialen Produkte sinkt. Sie folgen daher einer generalisierten Anrufung, die durch eine paradoxe Logik durchzogen ist. Denn »[w]enn jeder besonders sein soll, gleichen sich alle darin, sich von den anderen unterscheiden zu müssen « (Bröckling 2014: 16). Der Bedarf an Einzigartigkeit erzeugt so immer wieder Angebote, die eine künstlerisch, ästhetische Selbstinszenierung niedrigschwellig jeder und jedem ermöglichen, womit sie die Grenzen der Einzigartigkeit gleichzeitig verschieben. 
Das Image der Apps Daytum und Frickbits wäre ohne die biografischen Verweise auf ihre Macher*innen unvollständig. Bei beiden handelt es sich um Produkte eines Innovations- und Vermarktungsprozesses, der unmittelbar auf Ästhetisierungen und der Inszenierung künstlerisch-kreativen Handelns basiert. Kongruent zu der seit den späten 1960er Jahren anhaltenden Transformation der modernen Unternehmen zu kulturellen und affektiven Orten, die über die Einbettung der Mitarbeiterin als interpretierendes und emotionales Wesen gleichermaßen das volle Spektrum ihrer Persönlichkeit in den Arbeitsprozess einzugliedern bemüht sind (vgl. Reckwitz 2011: 185), vollzieht sich auch eine Emotionalisierung der Arbeitsformen und der mit ihnen verbundenen Symboliken und Semantiken. Das kreative Spiel mit kollektivierten Daten ist so als ein Ausdruck der enthusiastischen Affizierung genuin technokratischer Arbeitsprozesse durch ein Subjekt zu deuten, das mitunter vom stereotypen Modell, künstlerischer, nichtentfremdeter, expressiver Arbeit angeleitet ist (vgl. Boltanski und Chiapello 2003). Im Medium populärer Apps wie Daytum oder Frickbits spiegelt sich, den Maximen der Designökonomie entsprechend, die Einsetzung einer durchlässigeren Grenze zwischen Konsumtion und Produktion produktiv wider, während die Bedeutung der Ware im gleichen Zug hinter die sinnlich affektive Erfahrung zurückgestellt wird (vgl. Reckwitz 2011: 187). In dieser Form der Ökonomie sind die affektiven Relationen einerseits intersubjektiv und andererseits interobjektiv strukturiert, da sie nicht nur als Mittel der Selbstinszenierung dienen, sondern auch auf die Herstellung "faszinierender Objekte durch faszinierte Subjekte« zielen (Reckwitz 2011: 196).

»Take a spin through Daytum to see charts and lists of the most mundane details of people's lives, that, in aggregate, become fascinating. $\ll^{52}$

»As a break from working through some of the heavy papers on mood metrics that I asked for and received, I started playing with Daytum, the place to make pretty, web-based charts from your data. $\ll^{53}$

Datenkünstler*innen wie Laurie Frick (die Programmiererin der App Frickbits), auf die sich diskursiv bezogen wird wenn es um eine Überbetonung der künstlerischen Seite in der sinnbildenden Kohärenz aus Unternehmer- und Künstlerfigur geht, stellt für diese Grenzverschiebungen ein eindrückliches Beispiel dar. Durch die Popularisierung und Verbreitung einer unkonventionellen und datenbasierten Selbstexpressions-App einerseits, bei gleichzeitiger Kultivierung von Alleinstellungsmerkmalen andererseits. ehacker.com/5-242319/six-easy-ways-to-graph-your-life (zuletzt aufgerufen am 17.02.2017).

53 Post auf der Quantified-Self-Website mit dem Titel »Daytum for Pretty Tracking« von Gary Wolf. Quelle: http://quantifiedself.com/daytum/ (zuletzt aufgerufen am 17.02.2017). 
»[D]ata artists are hardly the first to express themselves through their daily activities - or to try to find meaning within life's monotony. ${ }^{54}$

»It's your life, your data - why not turn it into art? $\ll^{55}$

Diskursiv erscheint die Position der Datenkünstlerin als Ursprungsort einer speziellen Form der Selbstdarstellung auf der Meta-Ebene von Datensätzen, die die Funktionalität der Datensätze vornehmlich in ihrem Potential für die Erzeugung ästhetischer Produkte bewertet. Sie ist gleichzeitig Verfallserscheinung der bürgerlich-traditionalistischen Opposition von Ökonomie und Kunst zugunsten einer »Strukturähnlichkeit von ökonomischen und künstlerischen Praktiken« (Reckwitz 2011: 237).

Die Definitionsgrenzen der Datenkunst werden dabei aber zwischen ihren eigenen Selbstexpressionen und der app-basierten Massenproduktion künstlerischer Selbstinszenierungen stabil gehalten, womit auf den Umstand reagiert wird, dass die Aufmerksamkeitsökonomie »nichts mehr bestraft als Langeweile« (Bröckling 2014: 16). Um Originalität und Einzigartigkeit sicherzustellen scheut sie dabei auch keine medialen Brüche zu Lasten der Weiterverwendbarkeit und Anschlussfähigkeit, die mit dem Verlust der digitalen Form einhergehen.

Ihre Kunstkarriere begann Frick zeitgleich mit der Entstehung der QuantifiedSelf-Community (»2007-now working artist $)$ ) ${ }^{56}$ mit der sie eng verbunden ist. ${ }^{57}$ Ihre Arbeit fokussiert hauptsächlich auf die maßstabsgetreue Übertragung persönlicher Datensätze in den dreidimensionalen Raum physikalisch-haptischer Objekte. $\mathrm{Zu}$ ihren Arbeiten zählen etwa der Nachbau einer Time-Line aus Holzklötzen oder die Nachbildung eines Stress-Diagramms aus »handcut leather on stretched linen. $\ll^{58}$

Zwar erfüllen auch die aufgetürmten Bauklötze im weitesten Sinne noch eine heuristische Funktion als zumindest holzschnittartige Repräsentation gemessener Werte, allerdings macht das Beispiel deutlich, dass derartige Artefakte Ausdruck eines ästhetischen Selbstzwecks sind. Da es zu einer der zentralen Eigenschaften des Kunstbegriffs zählt, sich einer soziologischen Bestimmung zu entziehen, bzw.

\footnotetext{
54 Quelle: www.lauriefrick.com/ (zuletzt aufgerufen am 09.02.2017).

55 So lautet der Claim der iPhone-App >Frickbits«. Quelle: www.lauriefrick.com/ (zuletzt aufgerufen am 09.02.2017).

56 Aus den Biografischen Angaben von Laurie Frick. Vor 2007 war sie u.a. Co-Founderin eines Software-Unternehmens und als Marketing Director tätig. Quelle: www.lauriefrick.com/ (zuletzt aufgerufen am 09.02.2017).

57 Aktuelle Arbeiten von Laurie Frick werden regelmäßig auf der Quantified-Self-Website gepostet. Zudem präsentiert sie ihre Arbeiten auf den internationalen Konferenzen der Community.

Quelle: www.lauriefrick.com/stress-inventory/ (zuletzt aufgerufen am 09.02.2017).
} 
die Antwort auf die Frage danach was Kunst ist und was nicht, mit den Prämissen der Rezeptionsforschung gesprochen, natürlich ganz im Auge der Betrachter*innen liegt, soll an dieser Stelle gar nicht erst versucht werden wissenschaftliche Kriterien zu bemühen, um zu entscheiden ob eine Zurechnung dieser Selbstverdatungsspielarten zum Kunstspektrum angebracht ist oder nicht.

Vielmehr erscheint es sinnvoll in den Blick zu nehmen welche Funktionen die Selbstexpressionen im Diskurs erfüllen, die unter dem Begriff Kunst firmieren. Aus der Perspektive der Visuellen Soziologie sind dies zu allererst spezifische Differenzierungsleistungen, die durch die Kunstartefakte in entsprechende Interaktionsfelder oder Diskurse eingeführt werden, bzw. die nötig sind um diese Diskurse um sie herum zu bilden.

Abstrakte zeitgenössische Kunstbilder tragen durch ihre oft kryptische Machart unweigerlich die Frage nach ihrer eigenen Deutungsweise vor sich her und werfen die weitergehende Frage danach auf, was das Objekt zum Ausdruck bringen soll, bzw. unter welchen Bedingungen es als Werk hergestellt wurde. Interpretationen sind hier zuvorderst Meinungen und müssen begründet werden. Damit wird über die Betrachtung des Bildes hinaus in der Kunst notwendiger Weise die diskursive Aushandlung von Deutungsoffenheiten des Interpretationsprozesses selbst in den Fokus der Betrachtung gezogen. Visuell ausgerichtete Kunst spannt also eine reflexive Ebene zwischen ansonsten latent ablaufenden Deutungsprozessen auf und lässt sich bedingt als ein Erkenntnisgenerierendes Werkzeug bezeichnen, dass immer wieder als Medium der Irritation tradierter Blickregime, Rezeptionsformen, medialer Dispositive oder alltäglicher Latenzen wirkt.

Obgleich der Begriff »Data Artist « im Self-Tracking-Diskurs den Bereich kennzeichnet, an dem das Spiel mit den Repräsentationen von der mathematisch-analytischen Dimension des Self-Trackings abstrahiert und hier allenfalls aussetzt (»These pieces play on recent studies that show simple daily stress has a long-term effect on future chronic health problems $)^{59}$ und er einen Kristallisationspunkt für vorsichtige Skepsis in Bezug auf die Aussagekraft derartiger Datenpraktiken bildet, führen künstlerische Umgangsformen mit den eigenen Daten erstaunlicherweise nicht zu einer Irritation der verschiedenen Selbstverdatungspraktiken oder $\mathrm{zu}$ einer kritischen Thematisierung der persuasiven Effekte visueller Datenrepräsentationen im Allgemeinen.

Obgleich Daten das Potential zugesprochen wird künstlerisch rezipiert oder vergleichbar mit einer Modelliermasse in Kunst verwandelt werden zu können, bleibt die Beziehung vergleichsweise Rückkopplungsarm und ihre Eigenschaft als Werkzeug der Selbstexploration wird auf die Ebene der Produktion ästhetischer Artefakte verlagert, denen aber analog zu der Argumentationslogik in anderen Teilbereichen des Self-Tracking-Diskurses, also in Bezug auf vergleichsweise konven- 
tionelle Selbstverdatungsmethoden, eine ähnliche Überlegenheit als Erkenntnismodus attestiert wird:

»Data art can't capture the essence or totality of somebody [...] But she [Laurie Frick] believes personalized data art can accomplish something traditional art forms can't: It allows a viewer to see her nuances and idiosyncrasies in higher resolution-and to discover things she may have forgotten about herself or perhaps has never known. «0

Obgleich diese Argumentation die Präsentationsform in den Fokus rückt, stellt sie in Fragen des Erkenntnispotentials ebenso auf eine innere Objektivität ab, deren Grundlage die künstlerischen Materialien - also die Daten - beinhalten. In Abgrenzung zu künstlerischen Objekten ist aber auch zu beobachten, dass sich die Wahrheitsansprüche »normaler« Selbstvermessung durch die Abgrenzung zur Datenkunst zusätzlich erbauen:

»[...] I got to admit. Some of it may look pretty but l'm skeptical of it's point. The concept of let start collecting data so we can make something aesthetically pleasing. Seems to be trying to force a round peg into a square hole. The goal of collecting data is usually to draw conclusions, the goal of making art is usually to create some beautiful.«[sic! $]^{61}$

Die lediglich graduell geringer ausfallenden Ästhetisierungen die sich im Routinebetrieb regelmäßiger Meetups, Show\&Tells der Quantified-Self-Szene und noch weitaus stärker in der kommerziellen Peripherie massenhaft verbreiteter Self-Tracking Devices mit den traditionell eher nicht-ästhetischen Darstellungsformen akkumulierter Daten vermischen, bleiben von kritischen Kommentaren wie diesem allerdings meist gänzlich unberührt.

Etwas anders verhält es sich innerhalb des Self-Tracking-Diskurses mit Blick auf offensichtlich gescheiterte Visualisierungsversuche, die, insofern sie nicht undokumentiert bleiben, ironisch als Kunst thematisiert werden. Der Twitter-Feed "Accidental aRt ${ }^{62}$ sammelt Graphen, Kurven und Diagramme, die von Gary Wolf als Visualisierungen »that go »beautifully wrong " ${ }^{63}$ thematisiert werden. Obgleich auch hier die produzierten Datenrepräsentationen durch den offensichtlichen Mangel eines analytischen Nutzens ausschließlich auf der Ebene ihrer Visualität

60 Quelle: https://www.theatlantic.com/entertainment/archive/2015/05/the-rise-of-the-data-art ist/392399/ (zuletzt aufgerufen am 01.03.2017).

61 »Response«des Quantife-Self-Mitglieds Edward Bennett zu einem Artikel von Ernesto Ramirez mit dem Titel »Laurie Frick: Experiments in Self-tracking.«Quelle: http://quantifiedself.c om/2014/07/laurie-frick-experiments-self-tracking/ (zuletzt aufgerufen am 15.02.2017).

62 Quelle: https://twitter.com/accidental_aRt (zuletzt aufgerufen am 15.02.2017).

63 Quelle: http://quantifiedself.com/2017/02/reading-81/(Anführungszeichen im Original. Zuletzt aufgerufen am 15.02.2017). 
kommentiert werden können und ein semantischer Etikettenwechsel des Artefaktes von wissenschaftlich zu künstlerisch die Folge ist, lenken diese "Unfälle« in der Datenauswertung den Blick reflexiv auf die Datenbearbeitung im Allgemeinen und zwingen im selben Modus zur Auseinandersetzung mit potentiellen Problemen der Datenverarbeitung:

»My personal title for this beautiful work of accidental art is: »Causation is not correlation $\ll^{64}$

Datenkunst erscheint in Relation zu der Herstellung von validem Wissen damit diskursiv auch als ein abseitiges Produkt ohne faktischen Informationswert. In Relation $\mathrm{zu}$ »traditionellen Kunstformen« wird sie unter ganz ähnlichen Bedingungen jedoch als eine Form der Selbstexpression diskutiert, die in ihrer Detailgenauigkeit und Tiefenschärfe den Informationswert anderer künstlerischer Ausdrucksformen überlegen ist.

\section{Daten-Kurator*innen}

Obgleich die unmittelbaren Bezüge zwischen Kunst und Selbstvermessung die Quantified-Self-Community zeit ihres Bestehens begleiten, taucht der die Kunst begleitende Begriff des Kuratierens erst in den Jahren nach 2014 auf. Trotzdem die Figur der Kuratorin durch die Kreativwirtschaft diskursiv noch weitaus stärker auf unternehmerisches Handeln festgelegt wird, wird sie im Zusammenhang mit Quantified Self allerdings interessanter Weise auch mit politisch-emanzipatorischen Rhetoriken verwoben und dies während der oft instrumentalisierte aber durch seine diskursive Diversität noch immer undomestizierte Kunstbegriff jeder politischen Implikation entbehrt und im eben beschriebenen Sinne auf die Ebene von Ästhetisierungspraktiken reduziert bleibt. Gerade weil das relativ plötzliche Auftauchen des Begriffs im Zusammenhang mit Selbstvermessung oft fraglos hingenommen wird und sich der Begriff schleichend auch in die wissenschaftlichen Beschreibungen des Phänomens übertragen hat ${ }^{65}$, ohne dass dies merklich zum Thema gemacht würde, scheint es vielversprechend, sich diesem Aspekt noch einmal etwas näher zuzuwenden.

Soweit es den kommerziellen Kulturbereich betrifft, versinnbildlicht sich in der Konjunktur des Kuratierens bereits seit längerem die zunehmende Verflech-

64 Quelle: http://quantifiedself.com/2017/02/reading-81/(Anführungszeichen im Original. Zuletzt aufgerufen am 15.02.2017).

65 So z.B. bei Lupton: "Self-tracking becomes performative, both for the insights that a selftracker may achieve about her or his life and in terms of the aesthetics of the data that she or he may be able to curate and the decisions the self-tracker makes about which information to voluntarily share with others, which to keep private and which to try to ignore or avoid« (Lupton 2016: 113). 
tung unternehmerischer Ordnungsstrukturen mit künstlerischen Tätigkeiten, wobei die etymologische Nähe der Begriffe Curare und Care implizit bereits auf die hiermit verbunden Aufgaben verweist. ${ }^{66}$ Nämlich die Organisation möglichst produktiver Bedingungen für die Entfaltung kreativer Potentiale, die Pflege der richtigen Beziehungen und die Zusammenstellung relevanter Artefakte. Der Begriff wird daher wie kein Zweiter durch den kommerziellen Kulturbetrieb vereinnahmt, um die produktiven Komponenten kreativer und nonkonformer Freigeistigkeit mit ökonomischer Affektkontrolle zu verschmelzen. Während der Begriff im Kulturbereich entrepreneuriale Managementpraktiken kennzeichnet, hüllt er in den Technologie- und Informationsbranchen die mitunter trockenen und monotonen Organisations- und Verwaltungstätigkeiten in ein künstlerisches Flair und verweist so nebenbei auf den zunehmenden Stellenwert ästhetischer Aspekte der digitalen Informationsverarbeitung: "The modern use of the curation metaphor draws upon the art world and suggests that a digital content curator engages in activities similar to those of a museum curator who scours the art scene, selects the finest works, arranges the pieces around a unified theme, provides a frame to understand the artists' messages, and then engages the public in a conversation around the collection« (Weisgerber und Butler 2016: 1342).

Der zunehmende Stellenwert des Begriffs im Kreis der Self-Tracker*innen zeigt in Verbindung mit anderen Bezügen zu ästhetischen Praktiken daher die Ubiquartität von (Selbst)Managementpraktiken in der Gegenwartsgesellschaft auf, lässt sich aber auch gegen jede ökonomische Verkürzung richten, die in der Selbstvermesserin nur eine technisch aufgerüstete Variante des Homo Oeconomicus sieht. Vielmehr macht der Begriff als Scharnier zwischen ästhetischen und ökonomischen Praktiken deutlich, dass sich die Selbstverhältnisse, die im Zuge der Selbstverdatung angestrebt werden, bei weitem nicht in puritanischer, unternehmerischer Rationalität erschöpfen, sondern auch eine Orientierung am libidinös besetzten Ideal der kreativen Künstlerin beinhalten, die sich erst im Schaffensakt selbst bestätigt (vgl. Reckwitz 2011:510). Die Fähigkeit individueller Begeisterungsfähigkeit für einzelne Tätigkeiten bei gleichzeitiger Bereitschaft jeden Enthusiasmus auf die semiotische oder physische Produktion von Objekten auszurichten stellt nach Luc Boltanski und Ėve Chiapello sogar eines der wesentlichsten Kennzeichen einer "projektbasierten Polis« dar (Boltanski und Chiapello 2003: 63), die als Ensemble von Tätigkeiten die »Differenzen zwischen Arbeit, Freizeit, zwischen Erwerbstätigkeit, Hausarbeit und ehrenamtlichem Engagement« im Zeichen eines post-fordistischen Arbeitsgeistes zum Verschwinden bringen (Bröckling 2007: 261). Aktiv zu sein bedeutet ihnen nach vor allem Projekte $\mathrm{zu}$ initiieren und an den

66 »The term >curate has a curious etymology, beginning with the Latin curas and evolving into root of the English word >cure«, or literally to stake care of«« (Weisgerber und Butler 2016: 1341). 
Projekten anderer zu partizipieren (Boltanski und Chiapello 2003: 156), womit sie das dominante gesellschaftliche Leitbild auf eine Art Ballancekünstlerin oder Flexibilitätsvirtuosin zuspitzen (Bröckling 2007: 263). Wie Bröckling bemerkt, lässt sich zwischen den theoretischen Konzeptionen der Projektarbeiterin von Boltanski und Chiapello sowie des unternehmerischen Selbst als zentrale Anrufungsfiguren in der westlichen Gesellschaft eine gewisse Überschneidung attestieren, da beide »die Transformationen des Kapitalismus mit denen der Subjektivierung parallel« führen, »ohne Letztere aus den Zwängen ökonomischer (Selbst-)Verwertungen abzuleiten« (Bröckling 2007: 266). Obgleich Boltanski und Chiapello die projektbasierte Polis luzide nachzuzeichnen vermögen und mit ihrem Fokus auf neoliberal ausgerichtete Managementliteratur ein ähnliches Analysematerial verwenden wie Bröckling und Reckwitz oder vor ihnen Rose, gehen sie allerdings nicht da$\mathrm{zu}$ über soziale Konfigurationen zu untersuchen, in denen diese spezifische Ratio ihren Impact hat und in der sie sich als Aktivitätsethos in gesellschaftlichen Praxen reproduziert (vgl. Bröckling 2007: 260ff.). Die zentralen Legitimationsformen, deren Wandel nach Boltanski und Chiapello, den Eintritt des Kapitalismus in eine neue Epoche charakterisiert, sind in den Selbst- und Fremdbeschreibungen moderner Projektwelten allerdings vergleichsweise mühelos auszumachen. So geben die verschiedenen Organisations- und Austauschformate der QuantifiedSelf-Community ein überdeutliches Beispiel für die Orientierung an Freiheits-, Emanzipations- und Authentizitätsversprechen ab, die diesen »neuen Geist« beschwören. Projektfokussierter Arbeit wird nicht nur eine sinnstiftende und erfüllende Eigenschaft zugeschrieben, vielmehr wird ihre potentielle Innovationskraft gleichermaßen als Mittel beschrieben, gesellschaftliche Veränderungen im Sinne der Allgemeinheit zu bewirken.

Insbesondere in den Jahren nach 2014 taucht der Begriff des Kuratierens im Quantified-Self-Kontext immer wieder als Hervorhebung der Methodizität von Auswahlprozessen auf, deren Anwendungsfeld über Künstler*innen und ihre Arbeiten hinaus auch Self-Tracker*innen und ihre Daten umfassen kann. Ein vergleichsweise naheliegendes Beispiel, stellt eine Ausstellung im Rahmen der Quantified Self Expo dar, die 2015 in San Francisco stattfand und einige Werke von Künstler*innen exponierte, die ihre Self-Tracking Techniken mit der künstlerischen Praxis verknüpfen. Kongruent zu zeitgenössischen Darstellungs- und Selbstdarstellungsformen der Kunst, werden die Self-Tracker*innen und Mitveranstalter Alberto Frigo und Jacek Smolicki, die sich nach eigenen Angaben seit 2004 bzw. 2009 mit Lifelogging-Daten beschäftigen, durch den Veranstaltungstext als »Co-Curators« ausgewiesen: 
»In the words of our co-curators, Alberto Frigo and Jacek Smolicki: Art of SelfTracking is an exhibition gathering a number of international artists who use different personal data tracking techniques in their artistic practices. ${ }^{67}{ }^{7}$

Wohingegen der Bezug zur Kunstsphäre hier noch offensichtlich ist und es nicht verwundert, dass über die Exponate und Darbietungsformen auch das entsprechende Vokabular des administrativen Kunstbetriebs importiert wird, verdeutlichen andere Beispiele sehr viel stärker die euphemisierende Rolle, die der Begriff in der punktuellen Aktualisierung künstlerischer Authentizität in grundsätzlich kunstfernen Kontexten einnimmt. Denn rhetorisch schlägt schon die bloße Verwendung des Begriffs eine Brücke in Richtung der positiv besetzten Stereotype künstlerischer Freiheit, in deren Licht die verhältnismäßig unspektakulären Organisator*innen oder Verwalter*innen dann als nonkonforme Kreativunternehmer"innen reinkarnieren.

Die semantische Doppelbelegung des Begriffs weist das Kuratieren darüber hinaus aber eben auch als eine Management-Praktik aus, durch die Personen, Objekte oder Sozialbeziehung in artifizieller Weise auf den Status sortierbarer Elemente reduziert und im Sinne einer Content Economy produktiv arrangiert werden können. Eine Ambivalenz, die sich auch in der Bezeichnung des europäischen Jahrestreffens der Quantified-Self-Community als »carefully curated unconference ${ }^{68}$ widerspiegelt, also in einem Leitsatz der schon das Zusammenbringen der Self-Tracker*innen zu den annähernd 70 Vorträgen und Diskussionen als verantwortungsvolle Analyse- und Verknüpfungsarbeit der Organisator*innen betont und die Self-Tracker*innen selbst als arrangierbare Objekte und Ankerpunkte von Entscheidungen rekonstruiert. Wohingegen das Format der »Unconference« die Verantwortung für das qualitative Produkt als Summe partizipativer Einzelbeiträge demokratisiert, obliegt das Privileg der steuernden Erzeugung eines gleichrangigen Austausches nur Einzelnen. Der Begriff des Kuratierens dient damit einerseits der Herstellung eines bestimmten Selbstbildes das dem Ethos der nichtentfremdeten expressiven Arbeit der Künstler*innen folgt (Reckwitz 2011: 194), wohingegen seine funktionale Komponente, also sein Verweis auf das Handlungsprogramm der Kurator*innen andererseits den zunehmenden Stellenwert unterstreicht, den die geschulte Arbeit mit Informationen

67 Quelle: Post von Ernesto Ramirez (Webmaster der Quantified-Self-Page) http://quantifiedse If.com/2015/06/art-self-tracking/ (zuletzt aufgerufen am 16.11.2016).

68 Quelle: Z.B. die Ankündigung der Europe Quantified Self Conference des Jahres 2015: http:/ /quantifiedself.com/-2015/09/announcing-2015-quantified-self-europe-conference-program/ (zuletzt aufgerufen am 07.09.2015). 
(d.h. vor allem Recherche-, Beurteilungs- und Selektionsfähigkeit) innerhalb der postmodernen Wissensgesellschaft einnimmt (vgl. Reckwitz 2011: 139). ${ }^{69}$

$\mathrm{Da}$ in der Gegenwartsgesellschaft Informationen und soziale Netzwerke als wichtiges Kapital fungieren, sind die gehandelten Produkte mitunter nicht von den Handelnden zu trennen (vgl. Bröckling 2007: 265). Entsprechend verbindet sich mit der Funktionsrolle der Kuratorin gleichermaßen Macht und Hierarchisierungspotential. So hat im Unterschied zu jenen, die sich in »diskontinurierlichen Rythmen der Projektplanung« (Bröckling 2007: 262) zusammenfinden, die Qualitäten von Projektleiter*innen, wer selbst »Gegensätze ausgleicht und unterschiedliche Menschen zusammenbringt, wer schließlich ergiebige von unergiebigen Informationsquellen $\mathrm{zu}$ unterscheiden vermag, seine Umgebung unentwegt nach innovativen Signalen abtastet und frühzeitig aussichtsreiche Kontakte ausmacht « (Bröckling 2007: 264). Die durch die Initiator*innen der Quantified-SelfKonferenzen gewählte Formulierung »carefully curated « spiegelt dies zunächst als reflektiertes Verantwortungsbewusstsein von Gleicheren unter Gleichen wieder. An anderer Stelle wird der Begriff durch die Community schließlich aber ein drittes mal erweitert und unter Betonung seiner sprachgeschichtlichen Mehrdeutigkeit noch expliziter als ein Modus hervorgehoben, in dem sich einer prinzipiell gebotenen Sorgfaltspflicht im Umgang mit Daten praktisch nachkommen lässt. Ein in diesem Kontext verwendeter Begriff des Kuratierens umfasst über die Herausstellung einer dezidierten Methodizität bei der Auswahl und Organisation von Objekten (1) und der Rekontextualisierung dieser vergleichsweise profanen Tätigkeit als Form künstlerischen Schaffens (2) dann zusätzlich eine politische Implikation (3), durch die die Datenpflege als Machttechnologie reflektiert wird.

\subsection{Datenmacht}

\subsubsection{Data is the new oil: Interdiskursbeziehungen zwischen Self-Tracking und Big Data}

»But instead of using the trite and troubling metaphor »data is the new oil«, he asked us to turn our attention in a different direction, to the notion of thinking about data as part of our commons. »Properly cared for and curated, it can benefit all of us, like clean air and clean water « « ${ }^{70}$

69 Reckwitz verweist hier ausdrücklich auf den Stellenwert den neue Technologien innerhalb der inzwischen als klassisch zu bezeichnenden Diagnosen einnehmen, dass die postindustrielle Cesellschaft maßgeblich durch wissensökonomische Arbeit mit Ideen und Informationen geprägt ist.

70 Quelle: Enrica Tanamachi aus einem Vortrag von Kevin Patrick, dem Direktor des »Center for Wireless and Population Health Systems at Calit2«, während des 2015 Quantified Self 
In diesem Zitat klingen in sehr eindrücklicher Weise auch noch weitere Aspekte an, die einen Einblick in das politisch-strategische Programm von Quantified Self ermöglichen und darüber hinaus Facetten des dort vorherrschenden Selbst- und Weltverständnisses offenlegen. Über den hier bereits mit einiger Distanziertheit verwendeten Bezug auf die populäre Phrase »Data is the new oil« wird in dem Symposium öffentlichkeitswirksam auf einen Bereich referiert, den man als Großdatenforschung oder mit aller Vorsicht als Big-Data-Diskurs bezeichnen könnte. Also als eine bisher nur in Ansätzen definierte Chiffre, um die sich Diskussionen und Praktiken unterschiedlicher Anschlussfähigkeit und unterschiedlichen Organisationsgrades zur

»Erfassung, Speicherung und Analyse eines großen und beliebig erweiterbaren Volumens unterschiedlich strukturierter Daten[...]. «11

gruppieren. Die Phrase hat sich während der letzten Jahre vor allem im medienwirksamen und öffentlichen Versuch von Manager*innen aus den Software-, Technologieberatungsbranchen und natürlich dem Datenhandel als eines der hervorstechendsten Ergebnisse etabliert, eine gemeinsame Sprache zu entwickeln, die die Möglichkeiten und den mit großen Datenmengen verbundenen Wert auf den Punkt bringt. Selbstbeschreibungen wie die eines entsprechenden Panels zur Big-Data-Metaphorik während einer O'Reilly Conference aus dem Jahr 2001 dokumentieren eindrücklich das übergreifende Interesse vor allem verschiedener wirtschaftlicher Akteure eine Bestandsaufnahme vorhandener diskursiver Ausdrucksformen zu entwickeln und darüber hinaus an der Etablierung ausdrucksstarker Formeln selbst teilzuhaben. ${ }^{72}$

Der Ausdruck "Data is the new oil« hat sich dabei zu einem diskursiven Zentrum einer ganzen Reihe ähnlicher Analogien entwickelt, die, wie z.B. »Data Mining«, gemessene Werte oder Kommunikationsspuren als natürliche Ressource darstellen, die sich im Namen des ökonomischen Wachstums ausbeuten lassen oder in Begriff sind zur wichtigsten Währung der digitalen Welt ${ }^{73} \mathrm{zu}$ werden. Als

Public Health Symposium. http://quantifiedself.com/2016/02/health-data-explorers-kevin-pa trick/ (zuletzt aufgerufen am 16.11.2016).

71 Horvath, Sabine (2013): »Aktueller Begriff - Big Data«, Wissenschaftliche Dienste des Deutschen Bundestages, Berlin. S. 1. Quelle: www.bundestag.de/dokumente/analysen/2013/Big_D ata.pdf (Zitiert nach Püschel: 2014: 3).

72 »Big data is a massive opportunity; we are searching for its identity and the language to define it.«Quelle: http://radar.oreilly.com/2011/o2/big-data-metaphor.html (zuletzt aufgerufen am 15.03.2017).

73 So z.B. in einem Vortrag von Meglena Kuneva, European Consumer Commissioner während eines »Roundtable on Online Data Collection, Targeting and Profiling « in Brussels am 31 März 2009: »Personal data is the new oil of the internet and the new currency of the digital world." 
euphorisch-alarmistische Zuspitzung vermengt der Satz den informationsgesellschaftlichen Hoffnungsträger des Datenbooms dabei in auffälliger Weise mit industriegesellschaftlichen Maßstäben und nimmt den Charakter einer Werbeformel an, welche die Daten zum Schmiermittel der vierten industriellen Revolution erklärt.

Die populäre und häufig zitierte Rede von Virginia Rometty (CEO bei IBM) vor dem Council on Foreign Relations 2013 Corporate Conference in New York ${ }^{74}$ stellt für diesen Aspekt eine wichtige Referenz dar. Darüber hinaus findet sich ein ebenfalls mustergültiges Beispiel in einem Image-Video der Firma Tresata:

»]ust like oil was a natural resource powering the last industrial revolution, data is going to be the natural resource for this industrial revolution. $\ll^{75}$

Daten werden im Big Data-Diskurs allerdings nicht nur als flüssiges Gold, sondern auch als Ocean, Deluge, Torrent, Waves und Streams beschrieben, ${ }^{76}$ womit sich der Fokus auf Gefahrenszenarien im Zusammenhang mit natürlichen Elementen erweitert und die Diskussion über die Problematiken einer Überversorgung mit Daten bildsprachlich an den Diskurs angeschlossen wird. Piero Scaruffi, Autor des Buches »History of Silicon Valley « problematisiert in einem vergleichsweise jungen Beitrag die Gleichsetzung von Daten als (Zwischen)Produkte von Datenpraxen mit generativen Eigenschaften und der Ausbeutung fossiler Rohstoffe:

»The difference between oil and data is that the product of oil does not generate more oil (unfortunately), whereas the product of data (self-driving cars, drones, wearables, etc) will generate more data (where do you normally drive, how fast/well you drive, who is with you, etc). $\ll^{77}$

Quelle: http://europa.eu/rapid/press-release_SPEECH-09-156_en.htm (zuletzt aufgerufen am 15.05.2017).

74 Der genaue Wortlaut variiert dabei von Quelle zu Quelle. Die Reichweite der Aussage ist jedoch offenkundig: Die Website SiliconAngle zitiert sie: »l want you to think about data as the next natural resource.« Quelle: https://siliconangle.com/blog/2013/03/11/ibms-ceo-say s-big-data-is-like-oil-enterprises-need-help-extracting-the-value/Ein Ebenfalls viel zitierter Beitrag findet sich im Forbes Magazin: Quelle: https://www.forbes.com/sites/robertlenzner/2 013/03/07/ibm-ceo-ginni-rometty-crowns-data-as-the-globes-new-natural-resource/\#49c7ab 641d60 (beide zuletzt aufgerufen am 15.05.2017).

75 Abhishek Mehta, CEO Tresata, 2013. Auf youtube.com inzwischen nur noch für Angemeldete Nutzer*innen verfügbar. (zuletzt aufgerufen am 01.08.2017).

76 Eine etwas allgemeinere Herausstellung von Analogien zwischen Daten und verschiedenen Flüssigkeiten findet sich auf dem Blog von Deborah Lupton. Quelle: https://simplysociology. wordpress.com/2013/10/29/swimming-or-drowning-in-the-data-ocean-thoughts-on-the-met aphors-of-big-data/ (zuletzt aufgerufen am 17.5.2017).

77 Piero Scaruffi, den eigenen Angaben nach: »cognitive scientist and author of »History of Silicon Valley« aus dem Jahr 2016. Das Zitat stammt aus einem Interview von seiner Webpage. Quelle: www.scaruffi.com/singular/bigdata.html (zuletzt aufgerufen am 17.05.2017). 
Über die Tatsache hinaus, dass moderne Mobilitätskonzepte im Zeichen von UberApp und autonomen Fahrzeugen neben Öl inzwischen noch Daten als zweites Antriebsmittel benötigen, werden die Daten in diesem Szenario nicht verbraucht, vielmehr zählt es zu ihren vorrangigsten Eigenschaften in ihrem Vollzug immer weitere Daten zu erzeugen. Gleiches gilt für die zirkulären Anwendungsmöglichkeiten von datenbasierten Mustern auf die Datensätze selbst.

»Aus der »Kombination bisher nicht aufeinander bezogener Daten« resultieren neue Daten, die daran anschließend zur erneuten Strukturierung und Formung des gesamten Datensatzes eingesetzt werden (können). Durch die Größe und Handhabbarkeit heutiger Datensätze wird es somit möglich, diese selbst als Crundlage neuer Datengenerierung und -kombination zu gebrauchen. $\ll^{78}$

Ausgehend von der voranschreitenden Verbreitung von Wearables, dem sich ausbreitenden Einfluss der Quantified-Self-Community und der kollektiven Erzeugung immer weiterer Datenaggregate (z.B. aus den Bereichen des participatory Healthcare usw.) schließt die Big-Data-Industrie die dezentrale Produktion von Self-Tracking-Daten in diese Rechnung mit ein. Stellvertretend schreibt etwa Tom Fawcett, der Firma Silicon Valley Data Science, in dem Artikel »Mining the Quantified Self «:

»With increasing numbers of qualified self participants collecting ever greater amounts and types of data, many people literally have more data than they know what to do with. " $^{79}$

Entsprechend konstatieren auch Beratungsfirmen aus den Data-Analytics- und Accounting-Bereichen, dass der zukünftige Erfolg der Informations- und Wertabschöpfung, vor dem Hintergrund einer ansteigenden Datenflut, die sich wellenförmig in immer weitere Geschäftsfelder ausbreitet und die mit ihnen verwobenen Verwertungsketten beeinflusst, vornehmlich an dem Grad der Unkonventionalität und technischen Neuartigkeit der Anstrengungen bemisst, die für die Extraktion der Informationen aufgewendet werden. Eigentümliche Verweise auf die Fördermethode des Frackings stellen indes einen Versuch dar, die bereits konventionalisierte Metaphorik des Data-Minings zeitlich zu überhöhen und Plädoyers für unkonventionelles Denken im Daten-Business durch die Verwendung unkonventioneller Analogien zu unterstreichen:

78 Horvath, Sabine (2013): »Aktueller Begriff - Big Data«, Wissenschaftliche Dienste des Deutschen Bundestages, Berlin. S. 2. Quelle: www.bundestag.de/dokumente/analysen/2013/Big_D ata.pdf (Zitiert nach Püschel 2014: 3. Zuletzt aufgerufen am 13.03.2017).

79 Aus einem Artikel mit dem Titel: »Mining the Quantified Self: Personal Knowledge Discovery as a Challenge for Data Science«, der 2015 in dem Journal Big Data erschienen ist: (Fawcett 2015: 249). 
»Technology watchers predict an even greater flood of business data soon. It will come from the Internet of Things (IoT) - a network of intelligently connected devices, machines, and physical objects with sensors that will produce waves of data across the entire business value chain. [...] Management accountants must emulate the strategic mind-set of the frackers, who went beyond the standard practices of their industry to extract something valuable. [...] Like oil and gas fracking, management accountants will only get back as much as they put into it in terms of expanding their Big Data and data analytics activities. $\ll^{80}$

Durch den mehrfachen Wechsel der diskursiven Register (vgl. Bröckling 2013: 184) aktiviert diese Analogie dabei wahlweise Aspekte dominanter Überfluss- oder Knappheitsszenarien.

Stärker als viele andere Verwendungen der Öl- oder Ressourcen-Metaphern knüpft das Beispiel damit an die Verwendung der Öl-Metapher des britischen Mathematikers Clive Humby aus dem Jahr 2006 an, der gemeinhin als Ursprung der Metapher gilt. Anders als die meisten gegenwärtigen Rekurse auf die Analogie, sollte sein Vergleich allerdings darauf aufmerksam machen, dass Daten zwar kostbare Potentiale beinhalten, dass sich der Nutzen allerdings erst durch aufwendige Formen der Bearbeitung ihrer »Rohform« oder die Transformation zu anderen Erzeugnissen einstellt. Gerade vor dem Hintergrund einer immer häufiger diskutierten Überversorgung mit Daten, die das Problemszenario einer Datenknappheit inzwischen ergänzt, stellt sich der entscheidende Unterschied zwischen Daten, Informationen und daraus extrahierbarem Wissen demnach wortwörtlich erst beim Wechsel des Aggregatzustands ein:

»Data is the new oil. It's valuable, but if unrefined it cannot really be used. It has to be changed into gas, plastic, chemicals, etc to create a valuable entity that drives profitable activity; so must data be broken down, analyzed for it to have value. ${ }^{81}$

Unabhängig davon in welcher Variante die Ressourcen-Metapher ausbuchstabiert wird, ob Daten also als etwas konstruiert werden dessen Wert sich, ähnlich wie fossile Brennstoffe, durch eine potentielle Endlichkeit bemisst, als ein wachsender Vorrat regenerativer Energien oder eine sich selbst unkontrolliert reproduzierende Masse, die eingedämmt und drainiert werden muss, ehe sie nutzbar gemacht werden kann; den verschiedenen Bildern ist gemein, dass sie Daten erstens zur Bedingung für das Florieren einer zunehmenden Anzahl von Wirtschaftszweigen erhe-

80 Aus der Studie: Data fracking strategy der Ph.D.-Studierenden und Tredata-Mitarbeiter Clen Gary und Micheal Alles in der Zeitschrift Management Accounting Quarterly (Gray und Alles 2015).

81 Michael Palmer, von der Association of National Advertisers, in Erweiterung des berühmten Zitates des Mathematikers Clive Humby. Quelle: https://www.theguardian.com/technology/ 2013/aug/23/tech-giants-data (zuletzt aufgerufen am 08.04.2017). 
ben, ihnen hiervon ausgehend eine immer größer werdende gesellschaftliche Relevanz bescheinigen und sie dabei drittens als substantielle, physikalische Entitäten konstruieren, die ihre eigentliche Besonderheit als immaterielles, informationelles Konstrukt ausdrücklich negiert. Für den populären Teil des Big-Data-Diskurses ist daher besonders typisch, dass er mit dem engen Fokus auf die Bedeutung von Daten und die zusätzliche Verengung auf Bearbeitungs- und Auswertungsstrategien das Vorhandensein von Daten als unvermeidlichen und faktisch gesetzten Umstand zum Ausgangspunkt nimmt.

In der kritischen Begleitung der Big Data-Entwicklung wurde entsprechend bereits mehrfach eine Art populärwissenschaftlicher Mentalitätswandel hin $\mathrm{zu}$ einer postitivistischen Denkökonomie konstatiert. Schon die etymologische Herkunft des Wortes "Daten«, welches in der lateinischen Sprache soviel wie »das Gegebene« oder »das Vorfindliche« bezeichnet (vgl. Püschel 2014:12), liefert, fernab von öffentlichkeitswirksamen Naturalisierungen in den Selbstdarstellungen von Big Data-Firmen, Hinweise auf eine problematische Grundhaltung, die den gesamten Diskurs immanent bestimmt. Ihre spezifische Geltung oder ökonomische Attraktivität erhalten Daten jeder Art vor allem durch die sprachlich transportierte Vorstellung, es "mit unverzerrt der Welt abgerungenen Fakten oder unzweifelhaften »Wirklichkeitsmolekülen« $\mathrm{zu}$ tun zu haben, welche unreflektiert zur Beschreibung und Auslegung der Gesellschaft« oder einzelner Phänomenbereiche und Marktsegmente, Konsument"innengruppen oder politischer Zusammenhänge herangezogen werden können. Insbesondere das sprachliche Insistieren auf die "maschinelle Erzeugung und Verwertung" suggeriert dabei ein Verständnis von Daten das mit universell gültigen Aussagen über die Welt gleichgesetzt wird (Püschel 2014: 14). Obgleich, anders als durch Püschel oder verschiedene weitere Autor"innen argumentiert, schon oberflächliche Blicke in den Big-Data-Diskurs aufzeigen, dass entsprechende Wahrheitsansprüche in der Regel nicht unabhängig von entsprechenden Plattformen und Datensätzen proklamiert werden. Zwar konstruieren die Big-Data-Narrative vieler Firmen und Expert"innen eine als gegeben annehmbare Realität, der Wahrheitsgrad der möglichen Aussagen über diese Realität wird aber diskursiv (z.B. entlang marktwirtschaftlicher Einzelinteressen oder individueller Professionen über verschiedene Diskurspositionen hinweg) zu einer Frage der richtigen Methodik und der verwendeten technischen Systeme erklärt. Nichtsdestoweniger kommt durch die mal implizit mal explizit verwendeten aber doch gemeinhin geteilten metaphorischen Sprechweisen, die Daten zu faktisch Vorfindlichem aufwerten, sehr deutlich zum Ausdruck, dass es sich in der Summe all dieser unterschiedlichen Varianten und Methoden um systemübergreifende Versuche handelt, einen allgemeingültigen Mechanismus der Realitätserforschung zu suggerieren (vgl. Püschel 2014: 17). 


\subsubsection{Data is not the new oil: Naturalisierung und Politisierung von Daten und ihrer Erzeugung}

Die schriftliche und mündliche Sprache ist voll von bildlichen Verweisen und mit ihr auf so essentieller Ebene verwoben, dass ihre Vermeidung selbst bei der analytischen Betrachtung von Metaphern Mühe macht. Nicht zuletzt aufgrund dieser Tatsache ist es erstaunlich, dass viele Metaphern unsichtbar oder vergleichsweise unfrequentiert bleiben, wohingegen etwa die Gleichsetzung von Daten mit Öl eine so offensichtliche Wirkmächtigkeit entfalten kann. Analytisch weisen Metaphern zuallererst die Gefahr von psychisch-interpretativen Kurzschlusserklärungen auf. Dabei scheint eine Analyse der Bedeutung einer Metapher kaum geleistet werden zu können insofern der analytische Rahmen nicht über die einfache Beziehung eines Bildes und seiner Quelle ausgedehnt wird. Zudem ließe sich auch die durch Foucault angestoßene Auseinandersetzung mit der strukturprägenden Kraft von Sprache auf beliebig vielen Metaebenen fortsetzen, insofern man fragen würde, warum er zur Beschreibung moderner (Selbst)Kontrollverhältnisse gerade das Bild des Panoptikums gewählt hat oder an anderen Stellen vom Archiv oder einer Archäologie sprachlicher Diskurse spricht.

Die Diskursanalyse weist selbst im Grunde kein gesteigertes Interesse an der Metapher auf, sondern behandelt sie lediglich in kategorischer Unterscheidung zu andern ansonsten aber analytisch gleichwertigen Begriffen. ${ }^{82}$ Dabei geht es ihr weder um eine Psychologisierung ihrer Urheber*innen, noch darum eine kausale Beziehung zwischen einer Metapher und ihrer Rezeption zu unterstellen. Sie versucht vielmehr »zu bestimmen, gemäß welchen Schemata (der seriellen Anordnung, der gleichzeitigen Modifizierung) die Aussagen miteinander in einem Diskurstyp verbunden werden können « und »wie die rekurrenten Elemente der Aussagen erneut erscheinen, sich auflösen, sich erneut zusammensetzen, an Ausdehnung oder Bestimmung gewinnen, innerhalb neuer logischer Strukturen aufgenommen werden, umgekehrt neue semantische Inhalte annehmen und untereinander partielle Organisationen bilden können.« Diese so freigestellten Schemata zielen dabei also weniger auf die Beschreibung der Gesetze der inneren Konstruktion der Begriffe oder ihre fortschreitende und einzelne Genese »im Geiste eines Menschen«, als auf ihre anonyme Verstreuung über verschiedenen Autor"innen und Textgattungen hinweg (Foucault 1981[1969]: 89). Die Diskursanalyse »verbindet die Konstanten des Diskurses« daher »nicht mit den idealen Strukturen des Begriffs, sondern [sie] beschreibt den begrifflichen Raster ausgehend von den immanenten RegelmäBigkeiten des Diskurses«selbst (Foucault 1981[1969]: 91). Wo die Betrachtung der

82 Foucault ordnet die Metapher in die Ordnung der Dinge dem letzten Punkt seines Schemas einer allgemeinen Grammatik zu. Diese besteht aus den Begriffsfunktionen »Attribution, Cliederung, Bezeichnung und Ableitung« (1981[1969]: 89). 
Metapher als Interpretation eines ursprünglichen Sinns immer tiefer in die strukturalistische Sprachanalyse führen und sich von der Bedeutung, die diese Metapher (nicht idealtypisch) sondern reell im Diskurs einnimmt, entsprechend weiter weg bewegen würde, untersucht die relationale Diskursanalyse sprachliche Verdichtungen, die einzelne Teilaspekte einer umfangreichen Argumentation hervorheben oder überbetonen und so zum Attraktor von diskursiven Anschlüssen machen. Insbesondere Metaphern weisen hier die Eigenart auf, dass sie vornehmlich metaphorische Antworten und Entgegnungen provozieren, womit sich auf Dauer eine symbolische Ebene vor den inhaltlichen (z.B. zitationsbasierten) Austausch schaltet und die Komplexität der Argumente enorm reduziert. Eine ihrer vordergründigen Funktionen im Diskurs besteht damit darin, die antizipierbare Form dessen auszudrücken was allgemein verstanden wird wenn diese oder jene Aussage gelesen oder gehört wird. Die Bedeutungsinterpretation bemisst sich dabei vornehmlich aus dem Kontext der Aussagen, ihrer Entstehensbedingung oder ihres Sprechortes und die Plausibilität bestimmter Metaphern beweist sich häufig erst an der Art und Weise wie sie im Diskurs aufgenommen, gelesen und weiterverwendet wird. Dies macht sie bereits zu einem bestimmten Ausdruck vorherrschender Denkmodi und verfestigt sie nichtzuletzt auch weiter im diskursiven Vollzug. Der Grad der Indexikalität der Aussagen, d.h. der Grad der Chiffrierung einer Aussage und ihrer enthaltenen Verweise bestimmt dabei mit über die Feinmaschigkeit des Diskursnetzes und regelt als sprachliche Bedingung die Teilnahmemöglichkeit und den Zugang, in dem sie wie ein Hashtag wirkt. Die Tatsache, dass diese Bedingungen im Zusammenhang mit der »Data is the new oil«-Metapher vergleichsweise gering ausfällt, passt gut zu dem Umstand, dass sich mit der Metapher ein gesellschaftspolitisches Programm verbindet, dessen Reichweite eine ihrer wichtigsten Prämissen darstellt. Die Thematik wird durch die Verwendung von starken Bildern vereinfacht und in Relation zu gesellschaftlichen Sachverhalten gesetzt, zu denen bereits viele Vorläuferdiskussionen stattgefunden haben und von denen ein breiteres Verständnis vorherrscht. Es ist daher nicht nötig intentionalistisch zu argumentieren um ihren diskursiven Stellenwert als Sprache und Bild gewordenes Wissen zu beschreiben oder aufzeigen zu können, dass diese Bilder dabei meist von verschiedenen Interessengruppen unterschiedlich besetzt werden. Der politische Impetus der bildhaften Verweise im Self-Tracking-Diskurs eröffnet sich vielmehr in der allgemeinen Verwendung der Bilder auf die sie verweisen und ergibt sich aus ihrer unmittelbaren Kontextualisierung.

Die Bezugnahme von Kevin Patrick, während des Quantified Self Public Health Symposiums 2015, mit der er die Analogie zwischen Daten und Öl zwar in die Diskurssphäre der Selbstverdatung spiegelt, ihre Gleichsetzung allerdings verneint, ist ein deutlicher Hinweis darauf, dass die Quantified-Self-Community und ihre Peripherie den Entwicklungen in verwandten Datendiskursen aufmerksam folgt und 
mit eigenen Mitteln an diskursiven Verschiebungen oder Akzentuierungen arbeitet. Auf diesem Weg werden durch Querverbindungen zum Big-Data-Diskurs auch die dort dominanten Naturalisierungen aufgenommen, im Bereich der Selbstvermessung fortgeführt und weiter essentialisiert - wenn auch mit einer deutlich veränderten Zielrichtung: Wie in der Betrachtung der Rede von Kevin Patrick, bereits angeklungen ist, seien die Petabytes an dezentral erzeugten Daten, als »neue natürliche Ressource« wie »Wasser« oder »Luft « anzuerkennen und als Lebensgrundlage zu demokratisieren. Das Fördern, Verwalten und Pflegen von Daten wird hier semantisch also über den Gebrauch von Vokabeln aus dem Assoziationsfeld menschlicher Grundbedürfnisse mit einer fundamentalen Bedeutung ausgestattet und ihre uneingeschränkte Zugänglichkeit buchstäblich zur Lebensnotwendigkeit erklärt - was die gesellschaftspolitische Bedeutung der Öl-Metapher (z.B. in ihrer Bedeutung als natürlich begrenzten Garanten für ein spezifisches Industrialisierungsniveau) noch einmal übersteigt. Denn mehr noch als im Zusammenhang mit Öl stellt die Monopolisierung von Wasserreservoires weltweit ein starkes Politikum dar und wird weit häufiger durch zivilgesellschaftliche Akteure als Menschenrecht moralisiert. Eine sprachliche Parallelisierung von Daten und essentiellen Ressourcen wie Wasser, inkludiert damit semantisch generelle Aspekte moralisierender Nachhaltigkeitsphilosophie, Degrowth oder Postwachstumsansätzen und arbeitet so auch am Ausbau der industriekapitalistischen Raubbau-Assoziationen der Öl-Metapher aktiv mit:

»The next few years are going to be an exciting time in humanity's development, at precisely the time that our planet can no longer support the way we've done business since the 1800 's. $\ll^{83}$

Die Positionen der Quantified-Self-Mitglieder und ihres lebhaften Umfeldes (wie auch die der Akteure aus dem Big-Data-Diskurs) sind dabei allerdings selten einheitlich und durch inhaltliche Widersprüche und schematische Dissonanzen geprägt, die quer durch die wichtigsten Publikationsorgane und repräsentativen Funktionsrollen des Diskurses verlaufen. So positioniert sich etwa das Wired Magazin, dem Richard Barbrook nicht grundlos den Stellenwert des wichtigsten Sprachrohrs der Silicon-Valley-Ideologie bescheinigt (Barbrook und Cameron 1995), noch im Jahr 2014, also merklich spät, auf der Seite der Big-Data-Euphorie und attestiert eine chancenreiche Nähe zwischen digitaler Ökonomie und dem

83 Beitrag mit dem Titel »How to take back control of our data « von Michael Haupt in einer Diskussion unter dem Artikel »Data is the New Oil< - A Ludicrous Proposition. Natural resources, the question of ownership and the reality of Big Data des selben Autors. Quelle: https://medium.com/twenty-one-hundred/data-is-the-new-oil-a-ludicrous-propositio n-1d91bba4f294\#.q8fib8ain(zuletzt aufgerufen am 02.03.2017) 
rauen Abenteuerkapitalismus des 18ten Jahrhunderts: »Data in the 21st Century is like Oil in the 18th Century. ${ }^{84}$

Ganz allgemein scheint in der Community zudem eine mal mehr und mal weniger ausgeprägte Reflexion der gestaltenden Kraft der Sprache und spezifischer Techniken des Agenda-Settings auf. Ähnlich wie im öffentlichen Austausch verschiedener Big-Data-Firmen verdankt sich die häufige Verwendung von naturalisierenden Metaphern zwar bereits verselbstständigten Effekten und unreflektierten Nutzungsweisen, die in den diskursiven Marketing- und Politisierungsstrategien ihren Anfang nehmen. Jedoch finden sich auch Beiträge in denen sehr deutlich dafür plädiert wird über einen Wandel des gesellschaftlichen Verständnisses von Daten einen anderen Umgang mit Daten anzustoßen. Ein Bemühen um konkrete, rechtliche Regelungen ist entsprechenden Strategien der sprachlichen Diskursintervention (vgl. Foucault 1981[1969]: 86) dabei nachgeordnet und die Argumentation bezieht sich auf einen diffusen Standpunkt der zwischen Open-Data/Open-Access-Philosophie und individuellen Besitzansprüchen auf personenbezogene Daten wechselt. Eindrückliche Beispiele stellen die Blog-Artikel des Quantified Self Mitglieds Sara M. Watson dar, in denen sie etwa den bereits erwähnten Versuch verschiedener Akteure der Big-Data-Firmen, im Rahmen der O'Reilly Conference ${ }^{85}$ eine einheitliche Sprache zu finden, um das wirtschaftliche Potential von groß angelegten Datenanalysen zum Ausdruck zu bringen, als Artefakt eines industriellen Komplexes wertet, der die Perspektiven der verdateten Individuen selbst nicht genügend oder gar nicht berücksichtigt. So konstatiert sie in allgemeiner Form: "Many of the metaphors we have for personal data today come from the big data industry « ${ }^{86}$ und prangert die populären Daten-Metaphern als einen Vorgang der Entmenschlichung ${ }^{87}$ an, der schrittweise die Entmachtung und kommerzielle Ausgrenzung der Individuen zementiert:

»The dominant industrial metaphors for data do not privilege the position of the individual. Instead, they take power away from the person to which the data refers

84 Gastbeitrag von Joris Toonders des niederländischen Internet-Marketing-Unternehmens Yonego. Quelle: https://www.wired.com/insights/2014/07/data-new-oil-digital-economy/8 (Zuletzt aufgerufen am 02.03.2017).

85 Auf die Konferenz wurde weiter oben bereits Bezug genommen. Quelle: http://radar.oreilly. com/2011/02/big-data-metaphor.html (zuletzt aufgerufen am 15.03.2017).

86 Aus dem Beitrag: »Data is the New »_ «- Sara M. Watson on the Industrial Metaphors of Big Data.«Quelle: http://dismagazine.com/discussion/73298/sara-m-watson-metaphors-of-bi g-data/ (zuletzt aufgerufen am 15.03.2017).

87 »Most of the metaphors we use to talk about data in popular culture make sense to technocratic corporations and their leaders, those building and disseminating information technologies, but they are fundamentally dehumanizing.«Ebd. 
and give it to those who have the tools to analyze and interpret data. Data then becomes obscured, specialized, and distanced. ${ }^{88}$

Auch Jer Thorpe, auf den Sara M. Watson sich positiv bezieht und der für die Quantified-Self-Community allgemein als wichtiger Stichwortgeber sowie als Ikone der Daten-Visualisierung gilt, nutzt die Öl-Metapher in einem Artikel für das Harvard Business Review, um eine weitreichende Kritik am Big Data-Diskurs zu lancieren: »Perhaps the »data as oil« idea can foster some much-needed criticality«). ${ }^{89}$ Dabei perspektiviert er die industriellen Aspekte ebenfalls aus Richtung populärer Nachhaltigkeitspositionen, um sie anschließend in direkter Relation zu globalen ökologischen, ökonomischen und humanitären Krisen zu problematisieren.

»One of the places where we'll have to tread most carefully - another place where our data/oil model can be useful - is in the realm of personal data. A great deal of the profit that is being made right now in the data world is being made through the use of human-generated information. Our browsing habits, our conversations with friends, our movements and location - all of these things are being monetized. This is deeply human data, though very often it is not treated as such. Here, perhaps we can invoke a comparison to fossil fuel in a useful way: where oil is composed of the compressed bodies of long-dead micro-organisms, this personal data is made from the compressed fragments of our personal lives. It is a dense condensate of our human experience. $\ll^{90}$

Vergleichbar mit Kevin Patrick aktualisiert er dabei ökologiepolitische Standpunkte im Zusammenhang mit gängigen Formen der Big-Data-Geschäftsmodelle und stellt personenbezogene Daten so in die unmittelbare Nachbarschaft zu anderen gesellschaftlichen und ökologischen Bereichen, die aus Sicht kritischer Nachhaltigkeitprämissen einer sorgfältigen Behandlung bedürfen. Gegenüber den kurzsichtigen Antrieben des digitalisierten Industriekapitalismus, verorten sich derartige Argumentationen im Jargon kritischer Ökologiebewegungen und regenerativer Energiepolitik quasi auf der Seite des Planeten. Sein Vorschlag zur Bewusstmachung und Politisierung der Monetarisierung sozialer Aktivitäten im Netz basiert

88 Ebd.

89 Weiter heißt es an dieser Stelle: »Our experience with oil has been fraught; fortunes made have been balanced with dwindling resources, bloody mercenary conflicts, and a terrifying climate crisis. If we are indeed making the first steps into economic terrain that will be as transformative (and possibly as risky) as that of the petroleum industry, foresight will be key." Quelle: https://hbr.org/2012/11/data-humans-and-the-new-oil ([Anführungsstriche im Original]. Zuletzt aufgerufen am 15.03.2017). 
dabei in eigentümlicher Weise auf der metaphorischen Konstruktion einer biologischen Natürlichkeit von Ressourcen und ihrer anschließenden Übertragung in den Kontext aktueller Formen der digitalen Ökonomie. Wohingegen er die implizite Behauptung des »Data is the new oil«-Slogans, dem nach es sich bei Daten um einen per se gegebenen, fast schon materiellen Rohstoff handelt, als Wertschöpfungsprozess auf Kosten der Nutzer*innen digitaler Technologien desavouiert, treibt er die naturalisierenden Elemente der Analogie weiter voran - indem er neben der Makroskala Big Data auch ihre Mikroskala biologisiert und als inhärente Fragmente eines übergeordneten Ganzen beschreibt. Das erklärte Ziel dieser Strategie ist dabei, wiederum sehr ähnlich zu der Rede Patricks, eine neue Rahmung von »Daten«, als neue Form von Ressource zu erreichen, deren Besitz-, Verteilungs- und Verarbeitungsmodi vergleichsweise offen sind und die daher zum Gegenstand einer ethischen Erzeugungs-, Zugangs- und Verteilungsdebatte gemacht werden können und sollen. Anders als Patrick plädiert er indirekt zwar dafür Daten als ein öffentliches Gut aufzufassen, weicht im Zuge seiner Ressourcen-Metapher allerdings von einer oberflächlichen Ontologisierung »der Daten« ab und konzeptioniert über Begriffe wie »human«, »bodies « oder »personal lives« die Merkmalsträger*innen, ihre Körper und ihre Privatleben als genuinen Ursprung der Daten - aus dem er dann in der Folge ein Begründungsmuster für die formulierten Ansprüche ableitet.

»This re-framing of data into a human context is crucial. I believe there are three things we can do to make data more human [...]. First, people need to understand and experience data ownership. [...] Second, we need to have a more open conversation about data and ethics. [...] Finally, we need to change the way that we collectively think about data, so that it is not a new oil, but instead a new kind of resource entirely. $\ll^{91}$

\subsubsection{Doing Data}

Unter Berufung auf einschlägige Sentenzen der kognitionswissenschaftlichen Metapherntheorie George Lakoffs, denen nach diejenigen darüber entscheiden, was zu einem gegebenen Zeitpunkt als wahr erachtet wird, denen es gelingt sich mit ihren Metaphern diskursiv durchzusetzen, reflektiert Watson den öffentlichen Austausch über aktuelle Fragen des Umgangs und des gesellschaftlichen Stellenwerts von und mit Daten als einen vermachteten Strukturierungsprozess, dem sie ein eigenes appellatives Programm der bewussten Intervention durch das gezielte Vorantreiben einer körperlichen oder verkörpernden Metaphorik gegenüberstellt:

»We need a new framing of a personal, embodied relationship to data. Embodied metaphors have the potential to bring big data back down to a human scale and 
ground data in lived experience, which in turn, will help to advance the public's investment, interpretation, and understanding of our relationship to our data. [...] Embodied data metaphors put more control in our hands as individuals, capable of interpreting and intervening in our own personal data management. $\ll^{92}$

Das mittelfristige Ziel einer Imagination, in der die Verdatung von Körpern und die Verkörperung von Daten aufeinander zulaufen, soll dabei langfristig dazu führen, dass sich veränderte Werte oder Normen auch in veränderten Regelungen und Datenpraxen manifestieren.

»How we think about data - and more importantly what we do with it - will depend on the value systems that our conceptual metaphors capture and reify. « ${ }^{93}$

Obgleich ein personalisierter und stark körperfokussierter Bias für die QuantifiedSelf-Community konstitutiv ist und Watson selbst die Einschätzung vertritt, dass verkörpernde Metaphern innerhalb der Community verbreiteter sind als in anderen Bereichen, ${ }^{94}$ plädiert sie für eine weitere Radikalisierung der kommunikativen Verschmelzung von Daten- und Körperbegriffen, die sich konform zu den ausgeprägten nicht-diskursiven D.I.Y.- und Maker*innen-Aspekten der Community zusätzlich stark an einer offenen Innovationskultur ausrichtet - auch wenn sie die diskursiven Facetten höher hängt. ${ }^{95}$

»Tying data back to individuals, even at the metaphorical level, could change how we design the systems that manage it and policies that protect it. ${ }^{96}$

Diese Argumentation bildet das Verhältnis von großen Datenaggregaten und Individuen auf einem relationalen Raster aus oben und unten, groß und klein, fern und nah ab, bei dem die jeweils zweite Seite dieser binären Codierung die individuelle, selbstbestimmte Verfügung über Daten repräsentiert und auf diesem Weg eine Form von Ursprünglichkeit konstruiert, zu der die Daten »zurück« kommen

92 Ebd.

93 Ebd.

94 »The metaphors used in the Quantified Self community offer a more personal, autobiographical, embodied, or practice-oriented conceptual model of data.« Ebd.

95 So schreibt sie in der Beschreibung einer Breakout Discussion mit dem Titel »The Self in Data« während der Quantified Self European Conference 2013: »In my research on the QS community, I've found that we talk a lot about our technical requirements of data, and about how we want to use data. What we don't often talk about is what it means to know ourselves through data. This breakout is an opportunity to discuss what data tells us about ourselves and how we relate to our data."Quelle: http://quantifiedself.com/conference/Amsterdam-20 13/breakout-sessions.php (zuletzt aufgerufen am 15.03.2017).

96 Quelle: http://dismagazine.com/discussion/73298/sara-m-watson-metaphors-of-big-data/ (zuletzt aufgerufen am 15.03.2017). 
müssen. Der enteignenden Entfremdung großer, intransparenter und kommerziell verwalteter Datenaggregate in der Größenordnung von Big Data wird symbolisch so ein körperlich erfahrbarer Nahbereich gegenübergestellt, der die Daten »erden« kann und die Kontrolle in die »Hände« des Individuums legt. Die Interessen einzelner Träger*innen von Vermessungsmerkmalen würden demnach besser gewahrt, insofern sie entsprechend auch durch ein körperlich-metaphorisches Framing des Datenthemas im Diskurs repräsentiert würden. Watson's Rhetorik stellt dabei auf essentielle Verständnisse von Begriffen wie Körper und Mensch ab, womit sich gleichzeitig auch eine spezifische Form von Indifferenz zwischen Körperlichkeit und Selbst in diese sprachlichen Interventionsstrategien übertragen, die für den Self-Tracking-Diskurs in vielerlei Hinsicht symptomatisch sind (vgl. Abschnitt 3.1). ${ }^{97}$

Die schon bei Watson enthaltenen performativen Aspekte und die enge Kopplung des Datenbegriffs an "gelebte Erfahrungen« werden auch durch Jer Thorpe in einem durch die Quantified-Self-Community zitierten Web-Artikel weiter gesteigert. Ausgehend von der Beobachtung, dass sich im Wandel der Sprache gesellschaftliche Kräfteverhältnisse und technologische Entwicklungen nicht nur abbilden, sondern sie auch rekursiv durch sprachlichen Wandel stabilisiert und vorangetrieben werden, empfiehlt Thorpe, diskursive Strategien der gezielten Einwirkung auf populäre Imaginationsformen von »Daten« zusätzlich um eine syntaktisch-grammatikalische Verschiebung im Sprechen über Daten zu erweitern: „Let's turn data into a verb. ${ }^{98}$

Thorpe argumentiert, dass sich die Entwicklung des Wortes Data von seiner Verwendung als plurale Form des Wortes Datum bis zur Eingliederung in die syntaktische Klasse der Massensubstantive auf der Grundlage der zunehmenden Bedeutung von Daten, den Formen ihrer technologischen Verarbeitung und vor allem ihres generellen Quantums vollzogen hat. »Data« ist dieser Argumentation nach deshalb ein Massensubstantiv (und es wird deshalb von "data is the new oil« anstatt von »data are the new oil« gesprochen), da sich die Daten auf Grund ihrer Menge einer konkreten Zählung verwehren.

»As technology reacted to this dramatic shift in scale, so did language, and the word data found itself massified. ${ }^{99}$

97 Im Anschluss an Nicholas Rose und Carlos Novas ließen sich diese Formen der direkten Verbindung von Körper und Selbst als somatische Individualität bezeichnen (Rose und Novas 2000: 487).

Quelle: https://medium.com/memo-random/data-v-daoeod24777c\#.ukek1apkj (zuletzt aufgerufen am 15.03.2017). 
Ganz ähnlich wie Sara Watson, sieht auch Jer Thorpe das Potential über einen Wandel der Sprache, latente Machtverhältnisse zunächst offen zu legen und schließlich zu destabilisieren oder umzuformen. Die schon in der sprachlichen Verwendung inskribierten Asymmetrien sollen so aufgebrochen werden und die Ermächtigung des Subjekts überhaupt vorstellbar machen, dass in den Daten bisher nur als Objekt der Vermessung vorkommt.

»Making data a verb also exposes to us the power imbalances that have kept our collective endeavours drastically off-kilter. Grammatically speaking, data-as-verb would present a number of possibilities for subject/object combinations: I data you. You data me. We data you. You data us. They data me. They data us. We data them. ${ }^{100}$

Die unvollständige Aufzählung möglicher Deklinationen der Verbform des Begriffs Data klingt dabei mit der verkürzten Gegenüberstellung »They data us. We data them « atmosphärisch nach und stellt eine neue Artikulationsform für einen Kräfteausgleich in Aussicht, die allerdings weniger auf der Entzauberung der Datenmacht als auf ihre promethianische Beherrschung aus zu sein scheint. Ein "Doing Data« weißt in dieser Konnotation immer einen doppelten Handlungsbezug auf, der einerseits die Datenpraxis, d.h. die performativen Aspekte der Verdatung in der Analyse von Daten unterstreicht, gleichzeitig aber auch eine politische Handlungsoder Aktionsfähigkeit impliziert:

»By embracing the new verbal form of data, we might better understand its potential for action, and in turn move beyond our own prescribed role as the objects in data sentences. ${ }^{101}$

Obgleich sich der Big-Data- und der Self-Tracking-Diskurs letztlich noch vergleichsweise viele naturalisierende Metaphern teilen, so z.B. die Bezeichnung modularer, untereinander kompatibler, frei assoziierter oder formal gebundener Software-Komponenten als »Ökosysteme«, eröffnet die Biologisierung und »Humanisierung « ${ }^{102}$ von Daten eine Form der Betrachtung zahlenbasierter Repräsentationen auf empirische Körper- oder Sozialaspekte, die durch finalistische Ontologien wie »Data is the new oil« verdeckt werden. Die biologisierenden und verkörpernden Metaphern setzen dagegen an der Stelle zwischen individuellem Körper und aggregierten Entitäten - also auf der Ebene des Bevölkerungskörpers an und problematisieren sie als spannungsgeladenes Verhältnis zwischen einer inklusiven Interessengemeinschaft (»us«) und einem exklusiven Außen (»them«),

100 Ebd.

101 Ebd.

102 Hier in Bezug zu dem Begriff »data human« von Jer Thorpe. Ebd. 
dessen Reichweite über den Bereich kommerzieller Daten-Aggregations- und -Auswertungsverfahren hinausweist.

Charakteristisch für eine derartige Argumentation ist dabei, dass die Forderung nach einer Verminderung von Verdatungsprozessen als Diskursposition praktisch nicht vorkommt und lediglich verhindert werden soll, dass »die Daten« diskursiv nicht durch den Gebrauch von Metaphoriken überformt werden, die vom Standpunkt progressiver und optimistischer Technologiekulturen aus betrachtet als tendenziell rückwärtsgewandt und regressiv erscheinen. Die Zielvorstellung eines rhetorischen und praktischen Kräfteringens um die Hoheit über die gesellschaftliche Bedeutung von Daten und die (zumindest partielle) Kontrolle ihrer unmittelbaren Praxen imaginiert daher in vielen Fällen ein versöhnendes Zukunftsszenario in dem sich ungleiche Machtverhältnisse im Verdatungsprozess aufheben:

»In doing so, perhaps we can imagine a future perfect for data, where not only will they have dataed us, we will have dataed them. A future, perhaps, where we all data together. $\ll^{103}$

\subsubsection{Self-Tracking und Privacy}

Zudem ist der Anspruch alle Daten, die das eigene Kommunikations-, Surf-, Laufoder Shopping-Verhalten betreffen, einsehen und mit eigenen Mitteln bearbeiten zu können, keinesfalls gleichzusetzen mit dem was gemeinhin unter Datenschutz verstanden wird. In den weitreichenden Utopien vieler Self-Tracker*innen organisiert sich die Zukunftsgesellschaft geradezu auf der Grundlage einer weitreichenden Datentransparenz und stellt bereits heute die hinreichende Bedingung für die Teilnahme am gesellschaftlichen Leben dar: »Privacy is a negative right - it obliges others to leave you alone. ${ }^{104}$ Die prominenten Stimmen der Quantified-SelfCommunity radikalisieren damit Perspektiven die unter dem Begriff Post Privacy bereits seit längerer Zeit intensiv verhandelt werden und schlagen damit eine progressiv ausgerichtete Haltung hinsichtlich einer gesellschaftlich in mehrfacher Hinsicht unbestimmten und teils äußerst widersprüchlichen Praxis im Zusammenhang mit der Veröffentlichung persönlicher Daten im Netz vor. So schreibt etwa Kevin Kelly in einem Beitrag für das Wired Magazine mit dem Titel »Why You Should Embrace Surveillance, Not Fight It«:

\section{Ebd.}

104 Aus dem Webartikel "You are your Data « von Sara M. Watson. An späterer Stelle im Text heißt es: "Some suggest avoiding quantification as a subversive means of resistance, but that will be about as effective as hiding our heads in the sand." Quelle: www.slate.com/articles/technology/future_tense/2013/11/quantified_self_self_tracking_-d ata_we_need_a_right_to_use_it.html (zuletzt aufgerufen am 16.03.2017). 
»So far, at every juncture that offers a technological choice between privacy or sharing, we've tilted, on average, towards more sharing, more disclosure. ${ }^{105}$

Im Einklang mit der irritierenden Beobachtbarkeit dessen, dass die gegenwärtige Angst davor nicht wahrgenommen zu werden, die Angst vor der Überwachung gemeinhin $\mathrm{zu}$ überwiegen scheint (vgl. Weber 2017) und dass trotz einer allgemein eigentlich eher "positiven Bewertung von Privatheitsbelangen eine enorme Selbstauskunftsbereitschaft unter den Nutzer*innen von Online-Angeboten« (Püschel 2014: 11) zu verzeichnen ist, die zudem mitunter paradox erscheint (vgl. Barnes 2006), positionieren sich die Pioniere der Selbstvermessung mit einer eigentümlichen Form progressiver Kritik, die den gegenwärtigen Stand netzbasierter Überwachungstechnologien »umarmt «, dabei aber darauf abzielt eine zentralistische Machtanhäufung im gleichen Maße dadurch aufzuheben, dass die Zugänge zu den Daten kommerzieller Tracking-Unternehmen und staatlicher Überwachungsprogramme für jede Person offenstehen.

»I think people are at a point where they are sick of worrying about who is or isn't tracking their data [...] I say, run toward the data. Take your data back and turn it into something meaningful. ${ }^{106}$

Gerade weil dieses Zitat von Laurie Frick selbst lediglich affirmativ den Appell zu einer Flucht nach vorn mit emanzipatorischen Ansprüchen zu einer einheitlichen Formel vermischt, ohne $\mathrm{zu}$ benennen wie beide Fragmente konkret zusammenhängen, lässt es sich als ein gutes Beispiel für den apologetischen Daten-Bezug der Quantified-Self-Community in Fragen der gesellschaftlichen Bedeutung kontinuierlich wachsender Überwachungsinfrastruktur lesen, der einem essentialistischen und ethisch rein gehaltenen Verständnis von Daten, allenfalls die Gefahr einer missbräuchlichen Nutzung gegenüberstellt. Eine Haltung die sich nicht gegen Daten richtet, sondern den Daten entgegen geht, verspricht ferner von der Bürde zu befreien, ohne Unterlass Mutmaßungen darüber anstellen zu müssen, inwiefern das eigene Verhalten gerade getrackt wird oder nicht, da die Relevanz der Frage mit zunehmender Ausbreitung von Tracking-Systemen schlicht gegen null tendiert. Gesellschaftspolitische Eruptionen des Datendiskurses, wie etwa der 2013 durch Edward Snowden aufgedeckte Überwachungskomplex westlicher Geheimdienste, bleiben durch die Community dabei aber nicht unkommentiert und das Ausmaß der Überwachung wird keinesfalls geleugnet. So schreibt Kevin Kelly im bereits zitierten Artikel:

105 Aus einem Artikel des Wired-Magazins mit dem Titel: »Why You Should Embrace Surveillance, Not Fight It« von Kevin Kelly. Quelle: https://www.wired.com/2014/03/going-tracked-here s-way-embrace-surveillance/ (zuletzt aufgerufen am 22.03.2017)

106 Quelle: https://www.theatlantic.com/entertainment/archive/2015/05/the-rise-of-the-data-art ist/392399/ (zuletzt aufgerufen am 22.03.2017). 
»The internet is a tracking machine. It is engineered to track. We will ceaselessly self-track and be tracked by the greater network, corporations, and governments. Everything that can be measured is already tracked, and all that was previously unmeasureable is becoming quantified, digitized, and trackable. $\ll^{107}$

Über die aktive Einwirkung auf populäre Sprachbilder hinaus, die zur Beschreibung und Diskussion von datenbezogenen Themen verwendet werden, intervenieren wichtige Figuren der Quantified-Self-Community auch mit einer eigenen Position in den Überwachungsdiskurs, die zwar Forderungen nach einer Aufhebung von Datenmonopolen beinhaltet, über eine Kritik am Privatheitsbegriff allerdings eine eigene konträr liegende Vorstellung von informationeller Selbstbestimmung ${ }^{108}$ umreißt. Gemäß der Losung »You are your Data ${ }^{109}$ bedeutet den Daten entgegen zu gehen damit notwendigerweise dem Selbst entgegen zu gehen. ${ }^{110}$ Dieser kumulativen Logik nach führen mehr Daten über sich selbst dann zu besseren Möglichkeiten der Selbstbestimmung. Kritische Positionen werden so durch eine offensive Fortschrittsargumentation absorbiert, die eine kausale Beziehung zwischen Verdatung und Selbstentfaltungspotentialen konstatiert und gleichzeitig zukünftige innovations- und kreativitätbasierte Entwicklungspotentiale zu garantieren behauptet. In der Konfrontation der Bottom-Up-Perspektive des SelfTrackings mit der Top-Down-Perspektive des Big-Data-Diskurses werden zentralistische Trackingstrukturen so auf ihre Potentiale für die Generierung von Selbsterkenntnis abgeklopft und ihre Verwendung nach den Prämissen der Selbstverdatung in Aussicht gestellt.

Aus einem Artikel des Wired-Magazine mit dem Titel: »Why You Should Embrace Surveillance, Not Fight It« von Kevin Kelly. Quelle: https://www.wired.com/2014/03/going-tracked-heres -way-embrace-surveillance/ (zuletzt aufgerufen am 22.03.2017[Hervorhebung im Original]). Ein Programm, das auch von der Peripherie der Quantified-Self-Community begrüßt wird: »The first important step is for users to shift their thinking away from concerns of privacy and towards the value inherent in the data they generate on a daily basis. The loT and Quantified Self will help us reach this consciousness. «Aus einem Beitrag mit dem Titel »How to take back control of our data « von Michael Haupt in einer Diskussion unter dem Artikel »Data is the New Oik-A Ludicrous Proposition. Natural resources, the question of ownership and the reality of Big Data « des selben Autors. Quelle: https://medium.com/twenty-one-hundred/dat a-is-the-new-oil-a-ludicrous-proposition-1d91bba4f294\#.q8f1b8ain ([»loT « steht für »Internet of Things «] zuletzt aufgerufen am 02.03.2017).

Quelle: www.slate.com/articles/technology/future_tense/2013/11/quantified_self_self_trackin g_data_we_need-_a_-right_to_use_it.html (zuletzt aufgerufen am 16.03.2017).

110 Für eine alternative Deutung des Zusammenhangs zwischen Daten und Selbst, siehe den Artikel »You are your Data« von Deborah Lupton (2016b). 
»So while a world of total surveillance seems inevitable, we don't know if such a mode will nurture a strong sense of self, which is the engine of innovation and creativity - and thus all future progress. $\ll^{111}$

111 Quelle: https://www.wired.com/2014/o3/going-tracked-heres-way-embrace-surveillance/ (zuletzt aufgerufen am 22.03.2017). 


\section{Subjekt und Daten}

Subjekt und Daten stellen im Self-Tracking-Diskurs kein klar trennbares Verhältnis dar, oder fügen sich anders formuliert durch die performativen und interpretativen Einzelleistungen der Individuen diskursiv zu einer Subjektivität zusammen, die gleichzeitig das angestrebte Ideal und den aktuellen Fortschritt aller Versuche darstellt, diese Ideale zu erreichen. Im Folgenden soll anhand verschiedener Beispiele daher der empirische Charakter des quantifizierten Selbst hervorgehoben werden, das sich überall dort, wo es in unmittelbarem Zusammenhang mit SelfTracking-Daten steht, insbesondere durch einen Zustand permanenter Unfertigkeit, Fluidität und Uneindeutigkeit auszeichnet.

Entsprechend handelt es sich bei der Verdatung nicht einfach um ein Thema dieses Diskurses, sondern der Fokus auf empirisches Material, der auch der Infragestellung von Wissen und seiner gesellschaftlichen Funktion dient, bestimmt die formalen Strukturen des Diskurses in immanenter Weise mit. Spezifika dieses Diskurses liegen anders ausgedrückt darin, dass er erstens in besonders deutlicher Weise das Ziel der Subjektformung vor sich herträgt, da das Selbst zur Formation seiner Gegenstände zählt und er dabei zweitens die Erzeugung von Roh-Daten über sich Selbst als praktikablen Modus erscheinen lässt, den damit verbundenen Willen zum Wissen zu stillen. ${ }^{1}$

Auch wenn die Erforschung, Veränderung oder Verwirklichung des Selbst unzweifelhaft die Zielfolie dieser Datensammlungen darstellt, scheinen in der Summe der verschiedenen Praktiken, Konzepte und Diskussionen jedoch sehr unterschiedliche Selbstkonzepte auf, wobei eine sprachliche Definition oder Diskussion darüber, was das quantifizierte Selbst ausmacht, unterbleibt. Im Folgenden soll das Spezifikationsraster näher betrachtet werden, anhand dessen sich verschiedene datenbasierte Selbstkonzeptionen als Gegenstände des Diskurses herausbilden. $^{2}$

Zu einer dezidierten Auseinandersetzung mit radikalem Empirismus und mit ihm einhergehenden Diskursbildern, siehe den Sammelband » Raw Data is an Oxymoron« von Lisa Gitelman und Virginia Jackson (2013).

2 Um den ermöglichenden und eingrenzenden Bedingungen bei der Herausbildung diskursiver Gegenstände näher zu kommen, hat Foucault exemplarisch die Seele als Gruppe hierar- 
Dabei spielen eine ganze Reihe an Differenzierungen eine Rolle, die mitunter in Form von widersprüchlichen Konzepten nebeneinander existieren ohne explizit Bezug aufeinander zu nehmen. Dies betrifft zum einen Differenzkonzepte, die sich primär auf die Idee des Individuums stützen und hierüber sowohl generalisierte und nicht zuletzt statistisch geformte Menschenbilder durch die Betonung der Einzigartigkeit des eigenen Körpers und der individuellen Gefühlswelt konterkarieren (Abschnitt 3.1) und zum anderen diffus gehaltene, auf Zeitlichkeit basierende Unterscheidungen, die das Selbst aus Bezügen auf vergangenes, gegenwärtiges und zukünftiges Selbst definieren (Abschnitt 3.2). Hinzu kommen thematisch spezifische Datensammlungen- und Interpretationen, die das Selbst als ein Selbst der Routinen und Gewohnheiten, aber auch verborgener Determiniertheiten darstellen (Abschnitt 3.3). Die unterschiedlichen Konzepte werden im Folgenden unter Hervorhebung der thematischen oder epistemologischen Besonderheiten dargestellt im Zusammenhang derer sie im Diskurs auftreten.

\subsection{Das Selbst des Körpers und des Gefühls}

Unter dieser Kategorie lassen sich idealtypisch Ansätze summieren, die eine spezifische Form von Körperlichkeit in den Mittelpunkt stellen. Semantisch tritt das Selbst in diesem Kontext als Selbstbezug auf, der sich willentlich und geplant mit dem eigenen Körper und seinen einzelnen biologischen Funktionen (wie z.B. Blutzucker, Körpertemperatur, Herzratenvariabilität, Puls, Hautspannung, Atmung, Blutdruck, Gehirnaktivität, Gewicht oder Körperfett) beschäftigt. Es ist insofern der durch Rose und Novas bereits anhand neuartiger genetischer Diagnoseverfahren skizzierten »somatischen Individualität« ähnlich, die sich entlang körperli-

chisierter Fähigkeiten, den Körper als dreidimensionales Volumen von Organen, das Leben - d.h. die Ceschichte der Individuen als lineare Phasenfolge bzw. als Cesamtheit möglicher Reaktivierungen, zyklischer Wiederholungen, Korrelationsmechanismen, Systeme reziproker Projektionen - und das Feld zirkulärer Kausalität als Teilfragmente eines Spezifikationsrasters beschrieben, das die Diagnose verschiedener »Wahnsinnsarten « im psychiatrischen Diskurs des 19. Jahrhunderts angeleitet hat (Foucault (1981[1969]: 64). Obgleich unter Spezifikationen (anders als im psychiatrischen Diskurs) mit Blick auf den Self-Tracking-Diskurs kein kanonisiertes oder formalisiertes Wissen zu verstehen ist, ergeben sich dennoch eine ganze Reihe an auffälligen Ähnlichkeiten zwischen beiden Spezifikationsrastern, die möglicherweise daher rühren, dass auch die Definition des Wahnsinns nur über den Umweg einer Definition des Selbst und seiner Elemente als Orientierungspunkt und normatives Beurteilungsmaß möglich ist, bzw. es sich in beiden Fällen um eine Mischung aus psychischen und körperlichen Introspektionen mit sozialen Einflussfaktoren handelt, die jeweils über verschiedene Zeiträume (z.B. die Biografie einer Person) hinweg betrachtet werden. 
cher Merkmale definiert (Rose und Novas 2000). ${ }^{3}$ Sprachlich tritt dieses Verhältnis als die weit verbreitete und daher kaum mehr auffällige Differenzierung zwischen sich und dem eigenen Körper zu Tage. Ganz ähnlich auch im Englischen: »A year of tracking my body. ${ }^{4}$ Bereits die Formulierung mein Körper impliziert diese Trennung, da der Körper sprachlich vom Nominativ Ich differenziert, sein Besitz durch die Verwendung der ersten Person Genitiv allerdings gleichzeitig angezeigt wird. Der Körper kann so in Gänze oder reduziert auf einzelne Funktionen als eigenständige Entität mit eigenen Gesetzmäßigkeiten ausgewiesen werden:

»The application for which the chip is initially being designed is the continuous monitoring of circadian rhythms: our body's way of anticipating periodic environmental change. $\ll^{5}$

Dies ist insofern relevant, als dass die offenkundige Negation von subjektivem Empfinden eines der wichtigsten Kriterien darstellt, das die Selbstvermessung von anderen zeitgenösssischen Selbsttechnologien - z.B. aus dem Bereich der Esoterik oder der sog. Achtsamkeitsphilosophie - unterscheidet. Die spezielle Körperlichkeit des Self-Tracking stellt sich dementsprechend gerade dadurch her, dass z.B. Intuition oder ein >Gespür < für sich selbst einer Selbstbearbeitung durch Daten gegenübergestellt wird, die unabhängig von subjektiven Selbsteinschätzungen erscheinen. Subjektiven Kategorien wie Müdigkeit als Indikator zur Einschätzung von Menge und Qualität des Schlafs wird so etwa durch ein Set von Messwerten und Zeitepisoden und Vergleichszusammenhängen ersetzt, die objektive Erkenntnisse über Körperzustände in Korrelation mit Schlafdaten nahelegen.

D.h. Gefühle werden zu Indikatoren oder zu Zielgebieten technischer Interventionen (wie systematischer Erfassung, periodischer Beobachtung und Korrelationen) erhoben, darüber hinaus werden sie auf der Ebene möglicher Methoden der Selbstbeurteilung jedoch unter Hervorhebung der Mangelhaftigkeit der menschlichen Selbstbeurteilungsfähigkeit diskutiert:

»These weaknesses put us at a disadvantage. We make decisions with partial information. We are forced to steer by guesswork. We go with our gut. ${ }^{6}$

»The somatic individual [...] is also a subject of self-actualization, responsibility, choice and prudence - ethics that can only be operative in the light of a knowledge of one's bodily truth « (Rose und Novas 2000: 501).

4 Quelle: http://quantifiedself.com/2017/05/qs-amsterdam-2017-preview-can-picture-worth-th ousand-numbers/ (zuletzt aufgerufen am 01.10.2014).

5 Quelle: http://www.quantifiedself.com/2017/08/three-new-devices/ (zuletzt aufgerufen am 08.09.2017)

6 Quelle: www.nytimes.com/2010/05/02/magazine/o2self-measurement-t.html?_r=2\&ref=mag azine\&page-wanted=all (zuletzt aufgerufen am 22.07.2015). 
»Electronic trackers have no feelings. They are emotionally neutral, but this very fact makes them powerful mirrors of our own values and judgments. « ${ }^{7}$

»If you want to replace the vagaries of intuition with something more reliable, you first need to gather data. $\ll^{8}$

So konstatiert auch Deborah Lupon: »The visual image or data they generate are often privileged as more »objective« than the signs offered by the »real«, fleshly body« (Lupton 2013: 399[Hervorhebungen om Original]). »As one's bodily states and functions become ever more recordable and visualised via data displays, it becomes easier to trust the "numbers« over physical sensations« (Lupton 2013: 403).

Dennoch lassen sich diese Wendung gegen die subjektive Körper- bzw. Selbstwahrnehmung nicht im strikt reduktionistischen Sinne als eine Okkupation der individuellen Selbsterfahrung durch rationale Logiken beschreiben, die sich kongruent zu einer Ausweitung der »Kulturbedeutung des Kalkulativen « (Vormbusch 2012) vollzieht. Zwar erfolgt die Selbstwahrnehmung durch das Prisma numerischer Körperwerte mit dem ausgwiesenen Ziel, objektive Bewertungskriterien zu erzeugen, dennoch werden diese spezifischen Modi durch ebenso viele Gegenbeispiele konterkariert. Rationalisierungsthesen (vgl. Vormbusch und Kappler 2018) oder solche der Verwissenschaftlichung (Zillien und Fröhlich 2018) beschreiben daher sicher nur einen Teil der diskursiven Selbst-Konstellationen der immer parallel zu affektiven Diskursaspekten existiert.

Gefühle tauchen z.B. nicht nur als affirmativer Ausdruck der Effekte von Gefühlsrationalisierungen auf, sondern stellen, wie auch Pritz (2016) anmerkt, in vielen Fällen die Teilbereichsübergreifende Zielmarkierung des Self-Trackings im Allgemeinen dar; indem sie sich auf einen Zustand des Wohlfühlens bzw. Wellbeeings ausrichten (vgl. Abschnitt 6ff.).

Darüber hinaus trägt die an sie anschließende These einer Rationalisierung von Selbstbezügen auch dem Umstand kaum Rechnung, dass die Community in starkem Maße parallel eine emotional aufgeladene Inszenierung von Daten und ihrer Erhebungen, sowie eine Eventisierung des Self-Tracking betreibt. Aspekte wie dieser sind es letztlich, die das Self-Tracking als einen Ausdruck der kreativen Produktion und ästhetischen Rezeption (vgl. Reckwitz 2011: 145) von Selbstverhältnissen ausweisen, die sich bewusst von der Reproduktion der rationalistischen, lustfeindlichen Kultur des Kalkulativen abgrenzt, die - im Weberschen Sinne - auf einen formalen, bürokratischen und entemotionalisierten Persönlichkeitstyp ausgerichtet ist (Weber (1980[1922]): 31ff.). 
Obgleich die Vorträge und Talks der Community auf der Präsentation von numerischen Daten und entsprechenden Visualisierungen basieren und die Präsentationsregeln vor allem die Vorstellung präziser und zielgerichteter Projekte honoriert, mischt sich über die biografischen Narrative, die zuweilen von Verlustund Depressionsbewältigung, Sucht oder chronischen Krankheiten handeln, ein zusätzlicher Emotionalitätsaspekt in den Präsentationsprozess; "people respond emotionally to the data they generate from self-tracking, and their emotions may be highly evident in how they display or talk about their data (Lupton 2016: 111). Allerdings handelt es sich auch bei diesen Vorträgen ebenso wenig um unvermittelte Einblicke in das Privatleben der Vortragenden, wie die präsentierten Daten ein »Fenster zur Welt der sozialen Tatsachen« darstellen (Reichert 2018: 20). Als datenbasierte Selbsterzählungen sind sie keine faktischen Beschreibungen, sondern unterliegen Darstellungszwängen, Darstellungsmöglichkeiten, interaktiver Modellierung und interpretativer Rezeption. ${ }^{9}$

Die Objektivitätsaura numerischer Daten und die personalisierenden Narrative stehen dabei in einem eigenartigen Doppelverhältnis. Wohingegen die biografischen Zusatzinformationen die Anonymität numerischer Daten mit der faszinierenden Anziehung eines persönlichen Schicksals lebendig machen, objektivieren die Zahlenaggregate, Tabellen und Diagramme die subjektiven Selbsterzählungen.

Diese gegenläufige Entwicklung der emotionalen Aufladung formalistischer Praxen, bei gleichzeitiger Eindämmung der Bedeutung von Gefühlen im Alltag, lässt sich entsprechend eher durch eine analytische Perspektive beschreiben, die die Transformationen des Kapitalismus zwar mit denen der Subjektivierung parallel führt, letztere aber nicht aus den Zwängen zu ökonomischer (Selbst-)Verwertung ableitet (vgl. Bröckling 2007: 266). Als Auflösung der Grenze zwischen affektiven und rationalen Bereichen erscheint das Tracking daher nahezu paradigmatisch für neue Formen der Regulation von Sinneseindrücken und ihrer ästhetisierenden Verwertung durch unternehmerische Individuen (vgl. Pritz 2016: 144). ${ }^{10} \mathrm{Im}$ Mittelpunkt dieses Prozesses steht eine Subjektivität, die sich selbstbewusst gegen die emotionalen Hemmnisse und disziplinarischen Zurichtungen des maschinistischen Betriebskapitalismus in Stellung bringt, dabei aber ihre elementaren Prämissen enthusiastisch affiziert (vgl. Reckwitz 2011: 185ff.) indem sie die Differenz zwischen Arbeit und Freizeit zu annullieren versucht (Bröckling 2007: 261). pekte: Er ist Selbst Medium und Ankerpunkt für die Artikulation von persönlichen Problemen und Anliegen in einem gemeinschaftlichen Verhältnis, das in dieser Dimension dem Konversationsmodus von Selbsthilfegruppen sehr ähnlich ist: »These talks open a window on often very private aspects of people's lives« (Lupton 2016: 111). 
Damit wird deutlich, dass auch die Präsentations- und Darstellungsmodi dieses Diskurses (zumindest soweit es den organisierten Teil der Self-Tracker*innen betrifft) einen entscheidenden Einfluss auf die Subjektformung nehmen, der den rationalisierten Selbstverhältnissen täglicher individueller Datenpraktiken entgegenlaufen kann.

\subsection{Das Selbst der zeitlichen Entwicklung}

Dieser Selbstzuwendungsmodus erfolgt anhand der Betrachtung unterschiedlicher Lebensphasen, Zustände oder anderer episodischer Einteilungen, die sich dazu punktuellen Datenvergleichen im zeitlichen Verlauf bedienen. Sein bevorzugter heuristischer Operationsmodus ist der Längsschnitt in Verbindung mit verschiedenen Visualisierungstechniken. In Bezug auf Dauer, Datenmasse, Akribie und die weniger strikte Fokussierung auf numerische Daten weist diese Form des SelfTrackings viele Parallelen zu solchen Praktiken auf, die im Diskurs gemeinhin als Lifelogging bezeichnet werden (vgl. Abschnitt 2.4). Einteilungen dieser Art sind dabei notwendiger Weise aber immer nur idealtypisch. Meist lässt sich eine spezielle Selbstverdatungstechnik mehreren Bereichen zurechnen, wird in den entsprechenden Foren auch unter verschiedene Threads diskutiert oder zu verschiedenen thematischen Anlässen auf Meetups präsentiert. Auch die mit ihnen verbundenen Selbstkonzepte lassen sich nur idealtypisch differenzieren und kommen daher nicht immer überschneidungsfrei vor. So können Körpermerkmale auch im zeitlichen Verlauf beobachtet werden und entsprechend zwischen einer körperlichen Selbstbeschreibung im eben beschriebenen Sinne und einer Betonung früherer, gegenwärtiger und zukünftiger Zustandsbeschreibungen wechseln. Ein Beispiel, das sehr eindrücklich eine Überlappung von Körperbezogener Selbstvermessung und Langszeitbeobachtung mischt, liefert ein Beitrag der European Quantified Self Conference 2017 in Amsterdam:

»We weight ourselves, assess our body composition, and measure our waist and limbs. But would seeing how the shape of our entire body changed over time be a stronger motivator than numerical data? Psychological motivations aside, 3D body scanning may replace BMI as a go-to health measurement. It can help estimate body fat distribution, which correlates with cardiovascular disease and cancer risk.

On a fun note, $3 \mathrm{D}$ body scans could help us find better fitting clothes. ${ }^{11}$

Auch in diese Aussage flimmert der spezifische Modus auf, in dem die Mitglieder der Quantified-Self-Community zwischen ästhetisch spielerischen Ausdrucksfor-

11 Quelle: http://quantifiedself.com/2017/05/qs-amsterdam-2017-preview-can-picture-worth-th ousand-numbers/ (zuletzt aufgerufen am 11.09.2017). 
men und einer technologisch verwirklichten Selbststrenge changieren. Über die Artikulation und Präsentation von Enthusiasmus und persönlicher Faszination erscheinen die oftmals profanen Praktiken der Datensammlung und ihrer Auswertung, bzw. das disziplinierte Befolgen der hieraus destillierten Maßnahmen als freiheitliche Entscheidungen und als authentischer Ausdruck der eigenen Person, die gebotenen Falls aber zwischen spaßhafter und sachlich-professioneller Verdatung den Ernst ihres Anliegens nicht vergisst: »All this is good insights and fun but what I really get out this data is tracking results against goals. ${ }^{12}$ Lee Rogers (»Technologist, futurist, entrepreneur ${ }^{13},{ }^{13}$ der sich wie viele andere Self-Tracker*innen in Fragen der Datenvisualisierung an den ästhetisierenden Formaten Nicholas Feltrons orientiert, ${ }^{14}$ praktiziert eine Form des Self-Tracking, das in erster Linie darauf abzielt möglichst viele teils belanglos erscheinende Daten zu sammeln und durch anschließende Analysen selbstgesteckte Ziele zu erreichen.

So bewirbt auch das Quantified-Self-Mitglied Anthony Galli sein selbstentwickeltes »Personal Control Center« mit der Nutzungsempfehlung:

»Measure your personal stats on a month-to-month basis or from time-to-time so you may objectively see your self-improvement trends. ${ }^{15}$

In der Regel geht es hierbei um verschiedene körperliche oder psychische Zustände, deren Realisierung sich in doppelter Abhängigkeit von der Datengrundlage und der eigenen Motivationsfähigkeit darstellt. »While self-tracking is directed at other aspects of life and not only health-related metrics, the idea that collecting data on oneself is a primary means by which good health can be established and maintained is dominant in discourses of self-tracking and the quantified self. Indeed self-tracking represents the apotheosis of self-reflexivity in its intense focus on the

Aus dem Transkript eines Vortrags von Lee Rogers während eines Quantified-Self-Meetups in der San Francisco Bay Area im Jahr 2014. Quelle: https://digitallifediaries.com/why-annu al-reporting/ (zuletzt aufgerufen am 11.09.2017). Auf der Webpage www.quantifiedself.com heißt es über Rogers: »Lee Rogers has been collecting data about himself for over three years. The daily checkins, movements, and other activities of his life are capture by automatic and passive systems and tools. What makes Lee a bit different than most is that he's set up a personal automation system to collect and make sense of all that data. A big part of that system is creating an annual report every year that focuses on his goals and different methods to display and visualize the vast amount of information he's collecting."Quelle: http://quant ifiedself.com/2014/08/lee-rogers-annual-reporting/ (zuletzt aufgerufen am 11.09.2017). Quelle: http://leerogers.com/ (zuletzt aufgerufen am 11.09.2017).

»But I stumbled across Nicholas Felton who is creating personal annual reports since 2005 . It resonated with me on how to snapshot my progress in life and how to see better trends over the time of my life.«»Inspired by Nicholas Felton's »Annual Reports« (www.feltron.com) I started creating annual snapshots of my quantified self three years ago. «Quelle: https://digi tallifediaries.com/why-annual-reporting/ (zuletzt aufgerufen am 11.09.2017). 
self and using data about the self to make choices about future behaviours« (Lupton 2016: 12). Veränderungswünsche oder diffuse Zukünfte lassen sich dieser Logik nach dabei prozesshaft durch die Separierung von einzelnen Tasks erreichen, die einer Zielvereinbarung mit sich selbst ähneln:

»[...]step out of yourself and reflect. Analyze your data at set time intervals is a great way. Measure that up to what you want out of life. In the end, you will thank yourself. $\ll^{16}$

»There is a difference between goals and objectives. Coals are lofty concepts like sbeing a better person<. The magic happens when you translate goals into actionable objectives, like to be healthy, drink more water. To be more well rounded and wise. ${ }^{17}$

Ganz ähnlich wie in der praktischen Indifferenz zwischen Selbst und Körper als explizitem Modus der mit Self-Tracking verbundenen Selbstwahrnehmungsformen, erscheint das eigene Leben bei der periodischen oder permanenten Quantifizierung und Speicherung partieller Alltagsgewohnheiten, Körperwerte, zufälliger Ereignisse, Interaktionen und konsumierter Nahrungsmittel als eine verobjektivierte Biografie, die vom Selbst getrennt ist, vor deren Hintergrund sich aber gleichermaßen vergangene und zukünftige Stadien des Selbst ablesen oder prognostizieren bzw. erstreben lassen. Die relative Kontrolle per se unsicherer Zukünfte wird auf diese Weise zur ökonomischen Ressource (vgl. Amoore 2013: 19). Das Selbst existiert hier als potentiell endlose Anzahl (vor allem) früherer Versionen, die sich in der Bewertung des gegenwärtigen Selbst als Konkurrent*innen adressieren lassen und sich so in das kompetitive Verhältnis mit anderen Self-Tracker*innen einreihen - sei es in Form schlankerer oder produktiverer Selbste.

»Boost productivity: Focus on results, hold yourself \& your friends accountable, and work toward specific goals. ${ }^{18}$

Durch die Einteilbarkeit von Zeitintervallen bei beliebiger Hervorhebung einzelner Aspekte der täglichen Gewohnheiten können so Entwicklungen skizziert oder die Einhaltung aktueller Aufgaben überwacht werden:

16 Quelle: https://digitallifediaries.com/why-annual-reporting/ (zuletzt aufgerufen am 11.09. 2017).

17 Ebd.

18 Quelle: https://www.personalcontrolcenter.com/ (zuletzt aufgerufen am 16.03.2017). 
»I can track these on a daily basis, like if I have gotten enough water in that day or if I walked enough steps [...] I also have annual dashboards to show my progress for the year. $\ll^{19}$

Dem Lifelogging ähneln derartige Selbsterforschungs- und Selbstkontrolltechniken dabei in Bezug auf den inhärenten Hang zur Maximierung der Datensammlung. Anders als beim teils ziellosen Lifelogging, das sich nicht selten im Akt des Datensammelns erschöpft und dabei oft Terrabyte große Speicher mit weitestgehend ungenutzten Daten füllt, ${ }^{20}$ stellt ungezieltes und möglichst allumfassendes Sammeln von Daten allerdings keinen Selbstzweck dar, insofern es auch nur vage mit dem Ziel der Selbstverbesserung oder dem Erhalt gegenwärtiger Gesundheitszustände und Produktivitätsniveaus zusammen gedacht wird. Der prinzipiell kaum eingrenzbare Vollständigkeitsanspruch in der Datenerfassung ergibt sich hier aus der empiristischen Logik selbst und hängt insofern auch mit der zunächst ziellosen Erhebung von Daten zusammen von denen angenommen wird, dass sie die interessanten Erkenntnisse von selbst offenbaren insofern sie nur umfangreich genug sind. Wie Markus Unternährer (ebenfalls in Bezug auf Lee Rogers) schreibt, handelt es sich bei der möglichst vollständigen Erfassung aller Lebensbereiche und Tätigkeiten bzw. aller sonstigen in irgendeiner Weise erreichbaren Daten zudem

19 Quelle: https://digitallifediaries.com/why-annual-reporting/ (zuletzt aufgerufen am 11.09. 2017).

20 Das scheinbar ziellose Sammeln von Daten im Zusammenhang mit rigoros eingehaltenen Praktiken der Datenerfassung (wie z.B. der Erzeugung eines digitalen Fotos im regelmäßigen Intervall von 15 Minuten) wird nicht nur durch Presseartikel und Fernsehfeatures sondern auch durch die Self-Trackerinnen selbst im Zusammenhang mit Zwangsstörungen diskutiert, wenngleich derartige Vergleiche hier in der Regel nicht auf eine Pathologisierung der Lifelogger*innen hinausläuft, sondern im Cegenteil versucht wird obsessive Verdatung in die gängigen Rahmenerzählungen des Self-Trackings einzubinden. So z.B. in einem Post des Quantified-Self-Mitglieds Kitty Ireland auf dem Blog der Firma Saga:»Some people have very specific goals for self-tracking, like regulating blood sugar or understanding their moods. Others seemingly just want to collect data about themselves, and as much of it as possible. You might see them at Quantified Self conferences wearing multiple fitness trackers and an automatic camera or two. They're not just OCD [Obsessive-Compulsive Disorder - eine im ICD-10 geführte Form von zwanghafter Persönlichkeitsstörung, die sich durch Rigidität, Perfektionismus und ein ausgeprägtes Kontrollbedürfnis auszeichnet. Anm. Th. M.] (maybe a few are), they're making an open ended scientific inquiry.« Einen Vortrag des akkribischen Liefloggers Cathal Gurrin, der über einen Zeitraum von sieben Jahren während seines Alltags massenhaft Fotos aus der First-Person-Perspektive aufnahm, teasert Ireland mit den Worten an: ¿Gurrin offers two reasons for creating such an archive. The first is the reason most people take up health and fitness tracking - improved self-knowledge is a basis for making better life choices. The other use he points to is the creation of a digital memory, so you'll never forget anything, even years later. In this video he explains to the Economist how and why he records as much of his life as possible.«Quelle: www.getsaga.com/blog/finding-patterns-inpersonal-data/ (zuletzt aufgerufen am 04.08.2017). 
um eine »weise Vorsichtsmaßnahme. Da über die Art und Beschaffenheit zukünftiger Probleme Ungewissheit besteht, kann die Datensammlung nur unter Risikoübernahme eingeschränkt werden « (Unternährer 2016: 207). ${ }^{21}$

Ganz im Sinne der epistemologischen Kernprämissen der Quantified-SelfCommunity wird der Datensatz dabei mit zunehmender Größe mehr und mehr als externalisierter Wissenspool über das Selbst behandelt:

»We're trying to build a digital version of your life experience, which we can then use as a search engine of the self. « $^{22}$

Derartige Antworten werden in den Vorträgen während der Community-Events dabei nicht selten als Offenbarungen präsentiert, durch die Self-Tracker*innen unerwartet tiefe Einblicke in ihre eigenen Gewohnheiten erhalten haben. So schreibt auch Deborah Lupton in Bezug auf ein Beispiel aus dem Bereich des Nutrition Tracking: "The app then calculates not only the nutritional values of the food but provides what is described as »deep insights into eating habits, such as whether the user eats more nutritious food in the morning or evening and where their »weak points « lie. According to the website: »Other apps tell you about your food. We're telling you about yourself« (Lupton 2016: 10).

Damit beschreiben sie den primären Handhabungsmodus großer Datenaggregate, der sich vor allem dadurch auszeichnet, dass er einem radikalen Pargmatismus folgt und zuweilen theoriefrei funktioniert. Wichtiger als die konkrete Übersetzung von ausformulierten Vorannahmen in ein empirisches Ergebnis ist hier die Handhabung der Daten selbst:

»You don't have to start with an outcome in mind when logging personal data. If you know how to ask the right questions of raw data, you may find patterns you didn't expect. The beauty of having lots of different types of data about yourself is that you may uncover unexpected correlations. ${ }^{23}$

Damit teilt die Quantified-Self-Community Datenpraktiken, die in den computerbasierten Wissenschaften oder dem Big-Data-Diskurs inzwischen weit verbreitet

21 Mit stärkerem Fokus auf den Zusammenhang von Körper und Cesundheit schreibt auch Lupton: »They privilege an intense focus on and highly detailed knowledge of the body in which it is suggested that possession of this knowledge of one's body offers a means by which illness and disease may be prevented « (2016: 9).

22 Beschreibung der Lifelogging-Praktiken und -Ziele von Cathal Gurrin. Aus einem Artikel mit dem Titel: »Finding Patterns in Personal Data« des Quantified-Self-Mitglieds Kitty Ireland. Quelle: www.getsaga.com/blog/finding-patterns-in-personal-data/ (zuletzt aufgerufen am 24.03.2017).

23 Quelle: www.getsaga.com/blog/finding-patterns-in-personal-data/ (zuletzt aufgerufen am 24.03.2017). 
sind und skaliert sie auf die individualistischen Datensätze der Selbstvermessung herunter.

»I still have probably a few hundred thousand data points. And live on the premise that with enough data, answers present themselves. ${ }^{24}$

Die Annahme, dass sich durch die Erhöhung von Datenvolumen auch die Aussagefähigkeit über soziales Verhalten erhöhen kann, gipfelte im Jahr 2008 im vielbeachteten Ausruf des vermeintlichen Endes der Theorie:

»With enough data, the numbers speak for themselves. [...] Scientists are trained to recognize that correlation is not causation, that no conclusions should be drawn simply on the basis of correlation between $x$ and $y[\ldots .$.$] . There is now a better way.$ Petabytes allow us to say: »Correlation is enough «. We can stop looking for models. We can analyze the data without hypotheses about what it might show. ${ }^{25}$

Deratige Perspektiven, wie die des zeitweiligen Self-Trackers Chris Anderson, ${ }^{26}$ die ein Primat empirischer Daten in der Erkenntnisgewinnung in Aussicht stellen, erregen dabei vor allem das Interesse populärer Community-Repräsentant*innen. Die Silicon Valley Ikone Kevin Kelly, der auch an der Gründung der QuantifiedSelf-Community beteiligt war, pflichtet Anderson entsprechend bei:

»[...] I'm not entirely sure that these correlative systems are model-free. I think there is an emergent, unconscious, implicit model embedded in the system that generates answers. But the model's invisibility doesn't matter because they work. It is not the end of theories, but the end of theories we understand. $\ll^{27}$

Obgleich diese Aussage den euphorischen Grundtenor der These merklich dämpft und zu bedenken gibt, dass die Datenerhebungs-, Verwaltungs- und Analyseinstrumente nicht voraussetzungslos funktionieren, besteht insoweit Einigkeit mit dem pragmatischen Empirismus Andersons, als dass große Datensätze und zugehörige

24 Quelle: https://digitallifediaries.com/why-annual-reporting/ (zuletzt aufgerufen am 11.09. 2017).

25 »The End of Theory: The Data Deluge makes the Scientific Method Obsolete.«Quelle: www. wired.com/2008/06/pb-theory/ (zuletzt aufgerufen am 24.03. 2017). Erstveröffentlichung des Artikels war in der Printausgabe des Wired Magazine 2007/16.

26 Chris Anderson verbreitete am 16 April 2016 über seinen Twitter-Account die Nachricht: »After many years of self-tracking everything (activity, work, sleep) I've decided it's pointless. No non-obvious lessons or incentives «; und im Zuge einer anschließenden Diskussion einige Tage später: »Having now done this since 2009, I think any observable benefits long ago reached diminishing returns.«Quelle: https://twitter.com/chr1sa/status/721198400150966274 (zuletzt aufgerufen am 24.03.2017).

27 Quelle: https://www.edge.org/discourse/the_end_of_theory.html (zuletzt aufgerufen am 24. 03.2017). 
Analysesysteme auch dann noch nutzbar gemacht werden können, wenn sie nicht vollständig verstanden werden. ${ }^{28}$ Der Stellenwert der durch datengetragene Verfahren generierten Erkenntnisse wiegt damit auch im Self-Tracking-Diskurs höher als die Erkenntnisse über das (genaue) Funktionieren der entsprechenden Verfahren und das Zustandekommen der durch sie gewonnenen Erkenntnisse.

\subsection{Das Selbst der Routinen}

Self-Tracking dieser Art befragt die Routinen des Alltags auf ihren ökonomischen Gehalt und sucht nach Verbesserungsmöglichkeiten jenseits der plattgetretenen Pfade täglicher Gewohnheiten. Besonders charakteristisch sind seine Wiederholungszyklen während der experimentellen Selbstvermessung, aber auch bei der kontinuierlichen Aggregation und Visualisierung von Daten. Insbesondere die Langzeituntersuchungen basieren dabei häufig auf Annahmen über separierbare und durch Wiederholung herausstellbare Phänomene.

»People do things for unfathomable reasons. They are opaque even to themselves. ${ }^{29}$

Dieses Vorhaben wendet vornehmlich Methoden des Vergleichs auf, die auf die Beschreibung verschiedenster Tätigkeiten angewandt werden und hier (wiederum sehr nahe an den Zielen z.B. vieler Big-Data-Verfahren) nach Regelmäßigkeiten, Auffälligkeiten und Mustern suchen:

»Collecting data about yourself can uncover discrepancies between how you think you behave and how you actually behave. ${ }^{30}$

28 Ganz im Einklang argumentiert auch der Wissenschafts- und Technikhistoriker George Dyson in einem Artikel über Andersons These: »What's a model? We presume two requirements: a) Something that works; and b) something we understand. [...] Our large, distributed, petabyte-scale creations are starting to gasp reality in ways that work just fine but that we don't necessarily understand.«Quelle: https://www.edge.org/discourse/the_end_of_theory.h tml (zuletzt aufgerufen am 24.03.2017).

29 Eine sehr häufig zitierte Phrase des Quantified-Self-Gründers Gary Wolf. Quelle: www.n ytimes.com/2010/05/02-/magazine/02self-measurement-t.html (zuletzt aufgerufen am 24. 03.2017).

30 Aus einem Artikel mit dem Titel: »Finding Patterns in Personal Data « des Quantified-SelfMitglieds Kitty Ireland. Weiter heißt es dort: »You might intend to go for a run five times per week, but your fitness tracking data will tell you exactly how far and how often you actually run. An app like RescueTime can reveal how you really spend your time on your computer. You might be surprised by the hours spent surfing the internet.«Quelle: www.getsaga.com/ blog/finding-patterns-in-personal-data/ (zuletzt aufgerufen am 24.03.2017). 
Die epistemologische Basis dieser Verfahren bildet die Annahme biologischer, physikalischer aber auch sozialer Phänomene, die im Abseits des menschlichen Bewusstseins prozessieren und die durch den soziotechnischen Formalismus des Self-Tracking aufgedeckt und kontrolliert werden können.

Im Licht der Daten erscheinen diese Variablen dann zugänglich für programmatische Interventionen durch selbst- oder fremdbestimmte Verhaltensnormen:

»Raise well-being: Crow physically, mentally, and emotionally by using your Personal Control Center to form good habits. ${ }^{31}$

Für das Erkenntnisinteresse dieser Ausrichtung ist damit charakteristisch, dass sie durch die experimentelle Entwicklung und Anwendung von Selbstvermessungsverfahren versucht verborgene Einflussfaktoren zu identifizieren und durch entsprechende Visualisierungstechniken sichtbar zu machen, die bestimmte Lebensaspekte determinieren, Potentiale begrenzen oder auf andere Weise das Kontrollvermögen über Körper-, Geistes- und Lebensumstände einschränken:

»We're only at the very beginning of taking advantage of the ways graphics and visuals reveal our mental errors, our biases, our very bizarre behavior and our blind spots - to our own minds and to the situations of other people. $\ll^{32}$

"Some graphics and interactives use a slightly different technique to show us how fooled we are by our own minds: they put the user in the drivers seat. «33

Das dieser Form von Selbstvermessungen zugrundeliegende Selbst richtet sich dabei weit weniger auf ein konkretes Ziel aus. Vielmehr sollen seine Grenzen explorativ ausgelotet werden. Den Ansprüchen liegen also allenfalls allgemein formulierte Ziele wie Zeitersparnis oder eine Verbesserung der Gesundheit zugrunde. Übertragen auf die im letzten Abschnitt relevante Unterscheidung zwischen »Goals« und »Objectives« bedeutet dies, dass die Suchbewegungen dieser Kategorie darauf abzielen auf der allgemeineren Ebene der »Goals« Interventionsfelder zu identifizieren, die sich in einem zweiten Schritt als »Objectives« konkreter formulieren, operationalisieren und systematisch bearbeiten lassen.

31 Quelle: www.personalcontrolcenter.com/ (zuletzt aufgerufen am 16.03.2017).

32 Weiter heißt es bei der Designerin, Journalistin und Developerin Lena Groeger, die durch die Quantified-Self-Community in ihrem Newsletter »What we read« zitiert wird: »Our brains fool us all the time.« Quelle: https://www.propublica.org/article/how-information-graphics-r eveal-your-brains-blind-spots (zuletzt aufgerufen am 24.03.2017).

Aus einem Artikel mit dem Titel: »How Information Graphics Reveal Your Brain's Blind Spots« von Lena Groeger, auf den sich die Quantified-Self-Community in ihrem Newsletter »What we are reading « bezieht. Quelle: https://www.propublica.org/article/how-information-graphi cs-reveal-your-brains-blind-spots (zuletzt aufgerufen am 24.03.2017). 
"So with this insight, I reset my objectives for this year to complete a set number of tasks per day and $\frac{1}{2}$ of them need to be part of projects I deemed shigh priority<. With this, I believe that I will fulfill those big aspirations. ${ }^{34}$

Auch dieser Modus der Selbsteinwirkung stellt insofern eine von vielen Bestrebungen dar, die darauf abzielen körperliche Beschaffenheiten und den Wahrnehmungszugang (z.B. zu den Funktionen des Körpers) unter den »Vorzeichen von Selbsterkenntnis, Selbstverwirklichung und Erfolg als willentlich wähl-, form- und optimierbar« (Pritz 2016: 129) erscheinen zu lassen. Er folgt dabei deutlich einer Verkopplung sinnstiftender Projekte bei gleichzeitiger Projektierung des Selbst, die sich überall dort, wo sie einen derart explorativen Charakter aufweist, kongruent zu den Versprechen der Großdatenforschung, vornehmlich als Entdeckungsunternehmen unbekannter Lebensbereiche präsentiert.

»[...] fitness trackers are trying to tune their training regimes to their own body types and competitive goals, but they are also looking to understand their strengths and weaknesses, to uncover potential they didn't know they had. Self-tracking, in this way, is not really a tool of optimization but of discovery. ${ }^{35}$

34 Ebd.

35 Quelle: https://www.nytimes.com/2010/05/02/magazine/o2self-measurement-t.html?_r=1\&p agewanted=all\# (zuletzt aufgerufen am 16.03.2017). 


\title{
4 Der Datenhoheit den Kopf abschlagen ${ }^{1}$
}

\author{
»Multitudes are marching to the big ket- \\ tled drum.« \\ Johnny Cash
}

\subsection{Optimierung und Empowerment bei Quantified Self}

Obgleich innerhalb des Self-Tracking-Diskurses verschiedene Selbst-Konzepte gleichzeitig florieren - ist dabei nicht von der Hand zu weisen, dass die meisten Verfahren der numerischen Selbstformung mit Kategorien der Steigerung und Erweiterung operieren. Dies mag auch einer der Gründe dafür sein, dass das Phänomen des Self-Tracking oft synonym mit dem Begriff der Selbstoptimierung verwendet wird und die Nennung des Einen ohne die Nennung des Anderen inzwischen beinahe unvollständig erscheint. Insbesondere in Tagesmedien und Reportagen wird dieser Zusammenhang immer wieder aufs Neue thematisiert und damit gleichzeitig beschworen und vorausgesetzt.

Auch in der sozialwissenschaftlichen Literatur fungiert der Begriff der Selbstoptimierung als Chiffre für eine anhaltende Transformation der Gesellschaft und ihrer Subjektformen, die sich nach neoliberalen Prinzipien ausrichtet. »[T]ypisch für eine sozialwissenschaftliche und öffentliche Begriffskarriere« sind die angewendeten Konzepte und die Rekurse auf wissenschaftliche Theorien dabei sehr heterogen (Röcke 2017: 13). Mit Blick auf jüngere soziologische Analysen zum Begriff der Optimierung lässt sich aber grundsätzlich eine weite und im Allgemeinen »mit Verbesserung gleichzusetzende Definition von einer engeren Fassung des Begriffs unterscheiden, bei der eine Zuspitzung der Bedeutung in Richtung auf eine technisch induzierte und grenzüberschreitende, ja entgrenzende Verbesserung des Menschen und seiner Leistungsfähigkeit erfolgt « (Röcke 2017: 1).

Bereits die allgemeine Verwendungsform des Begriffs der Optimierung ist insofern instruktiv, als dass sie durch ihre Festsetzung auf einen bestimmten Modus der Selbstveränderung bereits eine grobe Unterscheidung zu anderen, vor allem historischen Formen der Einwirkung auf sich selbst unterscheidet und z.B. simplifizierende Rekurse auf Gemeinplätze, wie Foucaults Konzeptionen verschiede- 
ner Selbsttechnologien im historischen Verlauf, aus der Theoretisierung des SelfTracking-Phänomens ausschließt. ${ }^{2}$ Denn im vergleichsweise jungen »Begriff der Optimierung steckt ein Superlativ. Optimierung ist nicht dasselbe wie Meliorisierung« (Bröckling 2014:3) - sie unterscheidet sich also von den antiken Philosophien der Selbstpflege oder spirituellen Selbstfindungspraktiken dadurch, dass in ihrem Namen nicht versucht wird ein mit sich selbst identisches Selbst (vgl. Bröckling 2007: 35) herzustellen, sondern dass dieses Selbst nur im Wunsch nach Verbesserung existiert.

Der Optimierungsbegriff kennzeichnet in vielen Fällen aber auch eine starke Verkürzung der Selbstvermessungsthematik. So scheint über die Verwendung dieser teils mechanistischen und teils betriebswirtschaftlichen Vokabel insbesondere ein Zusammenhang zwischen Selbstbezügen (allen voran den technisierten) und den Funktionsweisen der modernen Ökonomie unterstellt zu werden - und dies ohne, dass diese These überall auch wirklich ausformuliert würde. Das Inbeziehungbringen von Self-Tracking und Optimierung wirkt daher mitunter reflexartig und nicht selten im Verbund mit einer schwachen Theoretisierung die lediglich im oberflächlichen Anschluss an einzelne Begriffe Foucaults einen allgemeinen gesellschaftlichen Optimierungszwang unterstellen. Zudem gehen Beiträge die den Optimierungsbegriff in den Mittelpunkt stellen meist über die Tatsache hinweg, dass der Begriff durch die Self-Tracker*innen selbst kaum verwendet wird. Jede so zugeschnittene Betrachtung des Self-Trackings legt damit unweigerlich eine Inkonsistenz zwischen den Fremdbeschreibungen und den Selbstbeschreibungen der Self-Tracker*innen offen, die ihre Tools vor allem als technologisches Rüstzeug für den Befreiungsschlag gegen Bevormundung und Herrschaft verstanden wissen wollen.

Einen Gegenpol zu der theoretischen Überformung des SelbstvermessungsPhänomens durch deterministische Optimierungstheorien bilden entsprechend explizite Plädoyers dafür »stärker die Eigenperspektive jener Akteure zu berücksichtigen, die mit ihrem Handeln Ansprüche auf eine autonome Selbstwertsteigerung verbinden« (Röcke 2017: 1). ${ }^{3}$ Hier ist z.B. an jüngere Beiträge Uwe

2 So z.B. die ideengeschichtliche Ursprungsforschung in der griechischen Antike. In diesem Zusammenhang kritisiert auch Lisa Wiedemann, dass in dieser Hinsicht insbesondere das Konzept der Selbsttechnologie »inzwischen zum Gemeinplatz einer kritischen Beschäftigung mit diesem Phänomen geworden zu sein« scheint, von dem aus das Konzept vornehmlich zur Aktualisierung historischer Vorformen der Selbstpflege genutzt würde ohne dass eine weitergehenden Aussage getroffen würde (Wiedemann 2016a: 67).

3 Auch diese Ansätze verzweigen sich bis weit in den Journalismus, die Ratgeberliteratur und die wissenschaftliche Peripherie: Hierbei ist an Bellinger und Krieger's »Cesundheit 2.0« (2014) aber auch an das Quantified Self Institute zu denken. So z.B. auch bei Straub, Sieben und Sabisch-Fechtelpeter, die einer anthropologischen Grundannahme folgend den Menschen als »homo modificans«, also als ein sich optimierendes Lebewesen operationalisie- 
Vormbuschs zu denken. Zwar streift auch Vormbusch mit einigen Rekursen auf die französische Soziologie der Kritik - insbesondere die Luc Boltanskis Thesen, die im Zusammenhang mit einer Expansion kapitalistischer Logiken in neue Gesellschaftsbereiche stehen (Vormbusch 2016), letztendlich operationalisiert er Self-Tracking allerdings als ein Sich-Selbst-Entdecken, das gerade nicht deckungsgleich mit Selbstoptimierung sei (Vormbusch 2016: 47). Ähnlich wie in Zillien und Fröhlichs (2017) phänomenologischer Herleitung konzeptioniert auch er die Selbstverdatung als Praktik, die darauf abzielt »Leiberfahrungen aus der Sphäre des Vorreflexiven zu lösen und innerliche Erfahrungsbestände in eine repräsentierbare Form zu bringen « (Vormbusch und Kappler 2017: 222). Im Ergebnis handele es sich bei Formen der Selbstvermessung folglich um Praktiken, die "zwischen Verdinglichung und Autonomiesteigerung changieren und dabei gleichermaßen Modi der instrumentellen Rationalität ${ }^{4}$ als auch Quellen für emanzipative Potentiale darstellen können (Vormbusch 2016: 47f.).

Der Diskurs des Self-Trackings mit seinen Teildiskursen und seinen Interdiskursbeziehungen ist so vielfältig und komplex, dass sich schnell analytisches Material zusammentragen lässt, das sowohl die Optimierungsthesen stützt, als auch Praktiken, Äußerungen oder Vermessungsprojekte zu Tage fördert, die einen eindimensionalen ökonomischen Reduktionismus unterlaufen. Dies allerdings jeweils um den Preis, die Heterogenität des Diskurses nur fragmentarisch und unter Hervorhebung der Extremwerte zwischen Optimierung und Emanzipation abzubilden oder es bei einer unentschlossenen Offenheit im Sinne einer Sowohl-Als-AuchArgumentation zu belassen. Eine Alternative offeriert die Fokussierung auf genau die eigentümliche Besonderheit, dass sich dieser Diskurs sowohl unter den Prämissen der Optimierung als auch der Befreiung adäquat beschreiben lässt. Weitaus vielversprechender als Optimierung und Emanzipation antithetisch gegeneinander auszuspielen ist daher die Klärung der Frage, in welcher Weise diese Modi der instrumentellen Selbstrationalisierung und der Autonomiesteigerung miteinander verbunden sind.

ren, zu dessen natürlichen Eigenschaften ein Streben nach Verbesserung zählt (Straub et al. 2012: 40f.). Kongruent argumentiert auch Meißner mit einer Trennung zwischen quantifizierenden Praktiken in »Selbsteffektivierung «-als Präzisierung des im Optimierungsbegriff enthaltenen ökonomischen Rationalismus - und der »Selbststeigerung« als einer »Form der Selbstoptimierung im Hinblick auf unbestimmte, fiktionale und prinzipiell schrankenlose Möglichkeiten des Selbstseins« (Meißner 2016).

4 Vormbusch bezieht sich hier auf die Konzeption von Quantifizierung als Kulturtechnik der Objektivierung und instrumentellen Beherrschung nach Adorno und Horkheimer (1969[1944]). Obgleich er den Ansatz an anderer Stelle als »kulturpessimistisch « bezeichnet (Vormbusch und Kappler 2017). 
Eine weniger kontrastscharfe Unterscheidung zwischen ökonomischen Strukturzwängen der Kapitalexpansion in die Sphäre des Ideellen (vgl. Boltanski und Chiapello 2003) auf der einen Seite und einer emanzipativen Praktik des SichSelbst-Entdeckens auf der anderen Seite, vermindert zudem die Gefahr die spezifischen Aspekte neuartiger Phänomene (wie des Self-Trackings) durch makroperspektivische Theorien zu überformen oder schlicht den Rechtfertigungsmustern der Akteure zu folgen (Röcke 2017: 8) und die Phänomenbeschreibung so in sich selbst zu begründen.

Anstatt also vergleichsweise deskriptiv die auffälligsten Selbstauskünfte und intentionalen Äußerungen der Quantified-Self-Community zusammenzutragen, erscheint es vielversprechender $\mathrm{zu}$ untersuchen wie sich die Paradigmen der Optimierung und der Emanzipation durch die unterschiedlichen Äußerungen und Praktiken als produzierter sozialer Sinn herausbilden. Ein Zugang der sich weder auf die Selbstauskünfte der Individuen fokussiert, noch deterministische Theorien anlegt, rückt gerade die Verbindung zwischen den individuellen Praktiken zur Selbstherstellung und den gesellschaftlichen Rahmenbedingungen ins Zentrum der diskursanalytischen Betrachtung sozialer Sinnbildungsprozesse und ihrer auffordernden und drängenden Wirkung auf die Begriffe die sich Individuen von sich selbst machen. Hierdurch gelingt es auch besser die relative Gleichzeitigkeit des Verhältnisses zwischen Diskurs und Selbst in den Mittelpunkt zu stellen. Denn weder der Diskurs, das Selbst noch die ihn begleitenden Praktiken sind einander vorgängig (Bröckling 2010: 24) sondern bedingen sich gegenseitig. Neben der induktiven Theoretisierung im Moment der Diskursbeschreibung versprechen hier erneut vor allem genealogisch-historische Bezüge und eine Schärfung der verwendeten Begriffe zur Klärung zu verhelfen.

So zeigt eine differenzierte Auseinandersetzung mit den Begriffen Optimierung und Emanzipation, sowie ihre geschichtliche Genese im Zusammenhang mit ökonomischem Wandel und sozialen Bewegungen, eine enge Verwobenheit beider Begriffe auf, die weit hinter den Self-Tracking-Boom zurückreicht. Um diese Verwobenheit klarer in den Blick zu bekommen ist es allerdings zunächst nötig die undifferenzierte Verwendung von Optimierung und Selbstoptimierung etwas zu Resystematisieren. Denn beide Begriffe werden häufig sogar in Form einer nicht näher erläuterten Aufzählung in der verdoppelten Schreibweise »(Selbst)Optimierung« (vgl. Röcke 2017: 1) verwendet. So z.B. bei Bröckling oder Duttweiler. ${ }^{5}$ Dies ist insofern erstaunlich, da man annehmen müsste, dass die Unterscheidung zwischen Optimierung und Selbstoptimierung für die an Foucault anlehnende Gouvernementalitätsforschung im Grunde den interessantesten Aspekt aus-

5 Schon der Untertitel des 2016 erschienenen Debattenüberblicks »Leben nach Zahlen« stellt die offene Frage ob es sich bei Self-Tracking um ein Optimierungsprojekt handelt oder nicht (Duttweiler et al. 2016). 
macht. Schließlich scheint die Unterscheidung gut geeignet um die Art und Weise zu beschreiben, in der sich die Arbeitswelt und mit ihr die Organisationsprinzipien und Selbstverhältnisse in den letzten Jahrzehnten gewandelt haben.

Stattdessen verwendet Bröckling den Optimierungsbegriff mit einer gewissen Selbstverständlichkeit zur Beschreibung per se unerfüllbarer Steigerungsanforderungen, die dazu animieren individuelle oder kollektive Leistungsmöglichkeiten auszureizen oder darüber hinauszugehen (Bröckling 2013a: 4). Stefanie Duttweiler verbindet die Untersuchung von Optimierungsanrufungen mit ihren inzwischen grundlegenden Arbeiten zum Konzept des Glücks als neoliberaler Regierungstechnologie (Duttweiler 2007). Neben zahlreichen (vor allem explorativ empirischen) Publikationen zum Thema Self-Tracking (Duttweiler et al. 2016, Duttweiler et al. 2016b, Duttweiler 2017) bzw. zu der Verbindung von Self-Tracking und Sport (Duttweiler und Gugutzer) ${ }^{6}$ konzipiert sie vor allem im 2016 erschienenen Artikel »Alltägliche (Selbst)Optimierung in neoliberalen Gesellschaften« (2016) (Selbst)Optimierung als schrittweise und längerfristig angelegte »Modifikationen der alltäglichen Lebensführung hin zu einem glücklicheren, fitteren oder gesünderen Leben « (2016: 27), die im Zeichen von Foucaults Regierungsbegriffen als Strategien der Fremd- und Selbstführung konzipiert werden. Als Ergänzung zur Fremdkontrolle laufen Selbstkontrolle und Selbstbestimmung somit klassisch gouvernementalitätstheoretisch lediglich auf veränderte Formen der Kalkulier-, Kontrollier- und Verwaltbarkeit von Individuen hinaus (2016: 32). Die Optimierung des Selbst ist hier nahe an einer Optimierung der Selbst- und Fremdzwänge konzipiert, lässt aber offen, wo genau die Unterschiede liegen und ob das eine Konzept dem Primat des anderen Konkurrenz macht, es sogar ersetzt oder es Schnittmengen zwischen beiden gibt und wie diese aussehen.

Ob sich das Phänomen des Self-Tracking schlicht im Optimierungsnarrativ erschöpft oder auch in anderen Hinsichten als neue Mess- und Bewertungspraktik moderner Lebensweisen zu verstehen ist bleibt für Duttweiler offen (vgl. Duttweiler et al. 2016).

Der Umstand, dass es bislang kaum einen gouvernementalitätstheoretischen Beitrag gibt, der eindeutig kennzeichnet worin der Unterschied zwischen Optimierung und Selbstoptimierung liegt, bzw. ob zweiteres genealogisch mit ersterem überhaupt im Zusammenhang steht, mag auch damit zusammenhängen, dass sich eine solche für die Gouvernementalitätstheorien im Grunde typische Transformationsthese nicht einfach auf den elementaren und meist bemühten Wandel der gesellschaftlichen Kontrollmodi von Disziplinierung hin zu passiven Formen der Steuerung von Selbststeuerung übertragen lässt. Zudem sucht man die Manuale,

6 Quelle: https://www.uni-frankfurt.de/57462629/Gugutzer-Duttweiler_Self-Tracking-im-Sport .pdf (zuletzt aufgerufen am 24.02.2018). 
Pläne und ausformulierten Strategien zur Schaffung des quantifizierten Selbst vergeblich, was für das gouvernementalitätstheoretische Forschungsprogramm ein Problem darstellt. Eine Analyse, die das Self-Tracking auf der Ebene der Beratungsliteratur oder verschiedener Strategien des Gesundheitswesens untersucht, wird inzwischen zwar schnell fündig werden und mit ganz ähnlichen Dokumenten konfrontiert sein, wie sie bereits in Bezug auf die aktivierenden Losungen der Fitnessgurus oder der Jobcenter freigelegt wurden; um dem plötzlichen Auftauchen dieser numerischen Selbstzuwendungen und ihrer selbstbewussten Erklärung zum Befreiungsinstrument auf die Spur zu kommen, setzt dieser aus den Agenturen auf die Individuen herabschauende Blick allerdings zu spät an. Man könnte sogar sagen, dass sich die Optimierung im Moment ihrer Verinnerlichung als Selbstoptimierung der Beobachtung durch die Gouvernementalitätsstudien entzieht und sie sich durch ihre Fokussierung auf die Top-Down-Installation von gesellschaftlichen Leitmaximen in diesem Fall um ihre Zuständigkeit bringt. Dies hängt zum einen damit zusammen, dass die Erfindungs- und Entwicklungsmodi der technologischen Selbstbeschreibungsverfahren innerhalb eines sehr viel breiteren und dezentraleren Diskurses situiert sind, innerhalb dessen Ideen und Instrumente BottomUp und ohne die kausale Ableitung aus Planung und Resultat hervorgebracht werden. Zum anderen hängt es aber auch mit dem Konzept der Selbstoptimierung im speziellen zusammen, dessen Ubiquität und Wirkmächtigkeit als Maxime der Selbstführung zum Zeitpunkt der Entstehung erster Selbstvermessungstools bereits soweit vorangeschritten war, dass die wörtliche Erwähnung dieses Konzeptes überflüssig erschien und innerhalb des Self-Tracking-Diskurses entsprechend erst als Reaktion auf die kritischen Impulse der journalistischen Öffentlichkeit und der universitären Wissenschaften aufgenommen wurde. ${ }^{7}$ Den Prämissen der Diskursanalyse entsprechend offenbart sich die hegemoniale Position eines Diskurses folglich häufig gerade in dem was nicht gesagt werden kann - oder was nicht der Rede wert erscheint. ${ }^{8}$

Um der Entwirrung dieser eigenwilligen Verflechtung aus Optimierung und Selbstoptimierung, Disziplinierung und Befreiung Vorschub zu leisten, widmet

Denn die wissenschaftlichen oder massenmedialen Fremdbeschreibungen des Selbstvermessungsphänomens vollziehen sich nicht ohne Kenntnis der organisierten Self-Tracker*innen. Vielmehr haben entsprechende Reflektionen ihrer öffentlichen Wahrnehmung in diesem Diskurs einen festen Platz. Sie rezipieren, diskutieren und kommentieren dabei nicht nur wie in der massenmedialen Öffentlichkeit und den angewandten medizinischen Wissenschaften aus den Bereichen des mobile- oder public health über sie gesprochen wird, sondern nehmen auch Bezug auf verschiedene Deutungsmuster die in soziologisch oder philosophisch grundierten Studien entstehen.

8 »[D]er manifeste Diskurs ist die repressive Präsenz dessen was nicht gesagt wird « (Foucault 1981:39). 
sich der folgende Abschnitt daher zunächst der Untersuchung des Optimierungsbegriffs in seiner natürlichen Umgebung: Der fordistischen Arbeitswelt. Der anschließende Abschnitt verhandelt dann die unter dem Topos Empowerment firmierende Subjekttransformation, die gleichermaßen als einer der Auslöser für die Krise des Fordismus und der maschinellen Arbeitsoptimierung gilt und den Grundstein für neue Formen von Arbeit und die dadurch erwachsenden Selbstoptimierungsanforderungen legt. Unter den Abschnitten 4.5-4.7 sollen die geschichtlichen Ausschnitte der vorgängigen Abschnitte dann dazu dienen den Modus zu erhellen, in dem sich Selbstoptimierung und ein spezielles Emanzipationsverständnis im gegenwärtigen Diskurs der Selbstvermessung verbinden.

\section{Zum Begriff der Optimierung}

Der Begriff der Optimierung, so wie er heute verwendet wird, ist ein Kind der Rationalisierungsbewegung die zu Beginn des 20 Jahrhunderts eingesetzt hat. Angeregt durch das »Scientific Management« Frederick W. Taylors (Wupper 2002: 97) und unter starkem Einfluss der akademischen Betriebswissenschaften entwickelte sich in vielen Fabriken eine zeitanalytische Betrachtung von Produktionsvorgängen (vgl. Wupper 2002: 99), die vor allem das Zusammenwirken von maschinellen und menschlichen Elementen in den Mittelpunkt stellt. Ihr Ziel ist eine konstante Gewährleistung der Produktion auf dem höchstmöglichen Niveau, die über gleichbleibende Rahmenbedingungen erreicht werden soll, d.h.über geringen Verschleiß oder einfache Ersetzbarkeit von menschlicher Arbeit und maschineller Komponenten auf der Grundlage kontinuierlicher Leistungsüberwachung. Dieses Standardisierungsprinzip umfasst sowohl technische Richtlinien als auch verbindliche soziale Verhaltensregeln wie Arbeitsanweisungen und Vorgabezeiten (Wupper 2002: 101).

Obgleich die tayloristische Betriebsorganisation geschichtlich weithin einen Bruch mit der militärischen Führung der Betriebe markiert, vererbten sich über die parallele Verwissenschaftlichung beider Bereiche allerdings im gleichen Zug andere Kontrolltechniken in die moderne Fabrik, die bereits seit langem in militärischen Institutionen erprobt wurden. Waren die Kasernen schon der Ort an dem die numerischen Thesen der Anthropometrie erstmals im großen Stil auf menschliches Verhalten übertragen und empirisch untersucht wurden (vgl. Abschnitt III. 1.1), profitierte die Standardisierung der Produktion nun von den Erfahrungen der Heeresforschung bei der Zergliederung menschlicher Handlungen in normierte Zeiteinheiten. Entsprechend macht ein Diskussionsbeitrag während der Jahreshauptversammlung des Verbandes der deutschen Ingenieure im Jahr 1913 deutlich, dass man die Schießordnung des Heeres, also jenes schrittweise Einüben vormals zergliederter Bewegungsabläufe, die auch Foucault in »Überwachen und Strafen« 
ausführlich beschreibt, ${ }^{9}$ für »nichts anderes « als für ein »Scientific Management « halte (Wupper 2002: 101). ${ }^{10}$

Neben Foucault ist zur Beschreibung dieser Verwissenschaftlichung disziplinarischer Sozialordnungen sicher die Wirtschafts-, Herrschafts- und Verwaltungssoziologie Max Webers zuständig, der selbst allerdings nicht ganz unbeteiligt an der Genese des Taylorismus war. Im 1921 erschienenen Standardwerk »Wirtschaft und Gesellschaft « schreibt er: »Die höchsten Triumphe feierte die darauf aufgebaute rationale Absicherung und Einübung von Arbeitsleistungen bekanntlich in dem amerikansichen System des »Scientific Management «, welche darin die letzten Konsequenzen der Mechanisierung und Disziplinierung des Betriebs zieht. Hier wird der psychophysische Apparat des Menschen völlig den Anforderungen, welche die Außenwelt, das Werkzeug, die Maschine, kurz die Funktion an ihn stellt, angepaßt, seines durch den eigenen organischen Zusammenhang gegebenen Rhythmus entkleidet und unter planvoller Zerlegung in Funktionen einzelner Muskeln und Schaffung einer optimalen Kräfteökonomie den Bedingungen der Arbeit entsprechend neu rhythmisiert. Dieser gesamte Rationalisierungsprozess geht hier wie überall, vor allem auch im staatlichen bürokratischen Apparat, mit der Zentralisierung der sachlichen Betriebsmittel [...] parallel. So geht mit der Rationalisierung der politischen und ökonomischen Bedarfsdeckung das Umsichgreifen der Disziplinierung als universelle Erscheinung unaufhaltsam vor sich und schränkt die Bedeutung [...] des individuellen differenzierten Handelns zunehmend ein« (Weber (1980[1922]: 686[sic!]). Diese Beschreibungen stehen dabei

So schreibt Foucault z.B. in Bezug auf ein preußisches Infanteriereglement: »Das Reglement sah sechs Zeitintervalle dafür vor, das Cewehr bei Fuß zu stellen, vier Zeiten zum spannen, 13 Zeiten, um es auf die Schulter zu legen « usw. (Foucault 1977: 198). Zur expliziten Orientierung Taylors an militärischen Vorbildern vgl. Giedion (1982: 123f.).

10 Aussagen wie diese, lassen auch die von Marx im Grunde durchgängig verwendeten Polemisierungen der Produktionsverhältnisse durch Militäranalogien in einem anderen Licht erscheinen, dennoch werden die durch Marx skizzierten Konzentrationen disziplinarischer Macht auf einzelne Führungskräfte, zumindest auf der Ebene der Beurteilung von Arbeitsleistung, durch diese neuen Techniken gerade aufgeweicht und einer anonymen numerischen Logik überantwortet. Das von Marx beschriebene militärische Organisationsprinzip der Fabrik gehört damit eigentlich eher zu den Elementen die im Rahmen einer betriebswirtschaftlichen Neuorganisation der Produktion sukzessive abgelöst werden. In das Kapital Band 1 schreibt er in Bezug auf die Äquivalenz von Militär und Fabrik: »Die technische Unterordnung des Arbeiters unter den gleichförmigen Gang des Arbeitsmittels und die eigentümliche Zusammensetzung des Arbeitskörpers aus Individuen beider Ceschlechter und verschiedenster Altersstufen schaffen eine kasernenmäßige Disziplin, die sich zum erwähnten vollständigen Fabrikregime herausbildet und die schon früher erwähnte Oberaufsicht, also zugleich die Teilung der Arbeiter in Handarbeiter und Arbeitsaufseher, in gemeine Industriesoldaten und Industrieoffiziere, völlig entwickelt« (Marx 1974[1867]: 447). 
weitestgehend kongruent zu einer ganzen Welle experimenteller »Einschließungsordnungen, die soziales Verhalten kontrollierbar und optimal nutzbar machen sollen« (Foucault 1977: 191). Obgleich sich entsprechende Analysen, wie z.B. Foucaults dezidierte Auseinandersetzung mit der Reformation des Strafsystems nicht immer unmittelbar auf die Fabrik ausrichten, ${ }^{11}$ besteht auch für sie die zentrale Funktion "nicht zuletzt in der Bindung der Individuen an die Erfordernisse kapitalistischer Arbeits- und Produktionsverhältnisse« (Saar 2003: 167f.). Foucault beschreibt diese produktive Wende disziplinarischer Institutionen im Rahmen einer »Mikrophysik der Macht«, die verschiedene Disziplinartechniken zum Einsatz bringt um »die Kontrolle und Nutzbarmachung einer Gesamtheit verschiedener Elemente« zu gewährleisten (Foucault 1977: 191). Die Disziplin organisiert hier einen »analytischen Raum«, um die Individuen kontinuierlich beobachten zu können bzw. ihre »Qualitäten und Verdienste zu messen « (Foucault 1977: 184).

Im Zuge der zeitlichen Erfassung, Zerlegung, Analyse und Neukomposition menschlicher und maschineller Bewegungen, die sich mit dem Vokabular Foucaults treffend als »soziale Orthopädie« beschreiben lässt (Foucault 2003 [1973]: 85), ergab sich dabei die "gleichmäßig rhythmische Arbeit« (Wupper 2002: 105) als universelles Optimalkriterium des Arbeitsablaufs, das sich numerisch in der geringstmöglichen Streuung der gemessenen Zeitwerte je untersuchtem Produktionsvorgang ausdrückte (Wupper 2002: 105). Nicht umsonst wird der Begriff des Taylorismus auch in seiner alltagssprachlichen Verwendung häufig "mit der Stoppuhr, sprich mit den Zeitmessungen am Arbeitsplatz identifiziert (Wupper 2002: 105). Die betriebswissenschaftliche Transformation der Fabrikarbeit läutet dabei allerdings nicht nur das Ende des unmittelbar militärischen Führungsstils in den Großbetrieben, sondern auch aller Effekte lückenhafter Planung ein, die sich - z.B. in Form von längeren Pausen - durch Unregelmäßigkeiten im Produktionsablauf von selbst ergaben: »For the industrial worker the new discipline of clock-regulated worktime meant an end to the longer traditional workday punctuated by periods of leisure; for the employer it meant the calculation of productivity in terms of hours« (Rabinbach 1992: 31f.). ${ }^{12}$

11 Schon das in diesem Zusammenhang am häufigsten rezipierte Kontrollmodell des Panoptikums, beschreibt Foucault in seinen Vorlesungen über »die Wahrheit und die juristischen Formen « 1973 als generalisierbare »Institution, die sich für Schulen, Krankenhäuser, Cefängnisse, Besserungsanstalten, psychiatrische Anstalten und Fabriken eignet (Foucault 2003 [1973]: 85). Er verfolgt damit sehr deutlich die These, dass auch »die Strafsysteme in eine bestimmte »politische Ökonomie« des Körpers einzuordnen sind« (Foucault 1977: 35ff[Hervorhebung im Original). Auch die Beschreibung des Cefängnisses als »Cesinnungswandelmaschine« (Foucault 1977: 162), denkt die ökonomischen Prämissen der Gefängnisreformation bereits mit.

12 Zitiert nach Herrmann (2002: 193). Wie einer von Taylors Schülern (Frank Bunker Gilberth) schrieb, lag das eigentlich Neue daran, »die Leistung jedes einzelnen Arbeiters genau zu mes- 


\subsection{Die Mechanik der Macht und die soziale Orthopädie des Taylorismus ${ }^{13}$}

Zur Gewährleistung der unterbrechungslosen Produktion auf Akkordniveau, richteten sich die von Taylor entwickelten und durch die akademischen Betriebswissenschaften verbreiteten Organisationsmodelle auch auf die Kontrolle der Funktionsbedingungen von Werkzeugmaschinen über längere Zeitintervalle. Zur Optimierung der Maschinenlaufzeiten über die Reduzierung von Verschleiß wurde dabei zunächst versucht im Verhalten der Arbeitenden jene Handbewegungen zu identifizieren, die den Maschinen am wenigsten schadeten, ehe sich diese Erhaltungslogik auch auf die Gesundheit des Menschen übertrug. ${ }^{14}$ Im Zusammenhang mit den Versuchen »einer möglichst ökonomischen Ausnutzung des Arbeiters und der Maschinen ${ }^{15}$ finden sich damit bei Taylor erstmals »Ansätze zur Operationalisierung von Gesundheit als Optimierungskriterium « (Wupper 2002: 103[Hervorhebung im Original]). Mit verschiedenen Messungen wird in der Folge versucht das optimale Verhältnis zwischen Belastung, Ermüdung und Arbeitspausen zu ermitteln. Das Optimum stellt also die größtmögliche Produktivität bei gleichzeitiger Wahrung der Gesundheit der Arbeitenden dar und ist eng verknüpft mit einer funktionalistischen Gesundheitsvorstellung, die Taylor von den Naturalisten des späten 18ten und frühen 19ten Jahrhunderts bezog. Die Vorstellung basierte nicht mehr auf der Annahme eines ontologischen Gesundheitszustands, sondern erhob die quantitative Erfassung und Relationierung von Merkmalen selbst zur Ontologie. Gemäß den in Abschnitt III. 1.2 beschriebenen selbstreferentiellen Normalisierungsmodellen ${ }^{16}$ streuen die empirischen Merkmale der Arbeitenden in ähnlicher Weise um einen Mittelwert, wie die Zeitintervalle der maschinellen Abläufe. Damit werden die betriebswissenschaftlichen Gesundheitsmaximen über den Import der Gaußkurve und die durch sie definierte Unterscheidung zwischen normal und pathologisch schon zur Zeit ihrer Entstehung auf elementare Weise mitbestimmt:

sen« allerdings in der kleinteiligen Zergliederung der Arbeitsleistung in »ihre einzelnen Elemente (Gilberth 1925: 19). Zitiert nach Herrmann (2002: 197).

13 Vgl. Foucault (2003[1973]: 85).

$14 \gg B i s$ weit ins 20. Jahrhundert hinein waren die Wissenschaften vom Leben geprägt vom Konzept der Organisation. [...]. Der Körper gilt als eine gegliederte Einheit, dessen Teile in einer angebbaren Lage- und Funktionsbeziehungen stehen, in Wechselwirkung treten und sich systemisch zu einer Einheit schließen« (Schäfer 2004: 334).

15 Taylor (1922: 67). Zitiert nach (Herrmann 2002: 195).

16 Das Ineinandergehen von normierenden und normalisierenden Praktiken verdeutlicht sich im betriebswissenschaftlichen Diskurs sogar explizit auf sprachlicher Ebene durch die meist fehlende Differenzierung: »Normalisierung und Normierung werden in den Texten der Rationalisierungsbewegung synonym gebraucht« (Wupper 2002: 104). 
In der Folge wird Krankheit nicht nur als eine quantitative Abweichung des Normalen definiert ${ }^{17}$, sondern auch das gebotene Maß an Gesundheit im Arbeitsprozess anhand dieser Orientierung an Durchschnitten begrenzt. Das Optimum fällt in dieser Logik mit der als natürlich angesehenen Normalität der Gaußkurve zusammen, wodurch es für die Betriebswissenschaften und die an sie anlehnenden wissenschaftlichen Zeitstudien möglich schien (Wupper 2002: 104), den Arbeitsprozess kalkulatorisch zu beherrschen in dem sie die Ausnutzung der gegebenen Arbeitsbedingungen auf einen fixen Normalzustand orientierten und so die optimale Arbeitsleistung zu ermittelten: "The language of labor is evolving into a language of labor power as a quantifiable force of production, localizable in the economics of energy distributed within the body and the psyche« (Rabinbach 1992: 31f.). ${ }^{18}$ Die Tatsache, dass sich insbesondere die sog. freien Handarbeiten einer entsprechenden numerischen Erfassung und Normierung entzogen, wird als einer der Gründe für ihre weitere Zergliederung und Standardisierung durch die Technologie des Fließbandes betrachtet, das insbesondere durch die steigende »Fordrezeption« (Wupper 2002: 106) Eingang in die Produktion erhielt.

\section{Maschinenbegeisterung}

Im Jahr 1922 veröffentlichte der »Autokönig« Henry Ford seine Autobiografie. Hier sind die wichtigsten Elemente dessen, was zwei Jahre später durch den deutschen Professor für theoretische Nationalökonomie Friedrich von Gottl-Ottlilienfeld als »Fordismus« in breite gesellschaftliche Diskurse Eingang fand, bereits angelegt (Saldern und Hatchmann 2009: 174). ${ }^{19}$ Allen voran die Einführung und Perfek-

17 Taylor definiert den Begriff der Gesundheit biologistisch als »normalen Zustand des Gewebes« (Wupper 2002: 103).

18 Zitiert nach Herrmann (2002: 194).

19 Nach anderen Quellen geht der Begriff auf Cramsci zurück. Ob der Begriff nun durch die Befürworter oder Opponenten des »Fordismus« geprägt wurde, mit Hilfe der Webapplikation Coogle-N-Crams, das dazu dient die Häufigkeit eines Begriffs in mehreren Millionen Publikationen zu eruieren, lässt sich für das Jahr 1924 ein erster steiler Anstieg der ansonsten gleichmäßig über dem Nullpunkt verlaufenden Kurve ausmachen, ehe sie mit dem Jahr 1933 absteigt. Hier bestätigt sich auch die These über die folgenreichste Fordisierungswelle im Jahr 1948 in Europa. Gleichzeitig zeigt eine auf die Ebene einzelner Begriffe zielende Analyse allerdings auch den Unterschied zwischen einer Diskursanalyse und dem auf was z.B. im Rahmen einer quantifiziereden Inhaltsanalyse geleistet werden kann. Denn wie sich z.B. durch Coogle-N-Grams leicht zeigen lässt, wurde der Begriff Fordismus während des NS in Deutschland kaum verwendet, trotzdem eine starke Orientierung an der Produktionsorganisation Fords und auch teils starke ideologische Überschneidungen bestanden. Aufgrund seines amerikanischen Ursprungs wurde der Begriff bis 1945 überwiegend wieder durch den Begriff der Rationalisierung ersetzt und wird erst in Mitten der Ruinen des NS wieder zur Benennung entsprechender Denkgebäude benutzt. Quelle: https://books.google.com/ngrams/graph?content=Fordismus\&year_start=1800\&year_end 
tionierung von Fließfertigungssystemen, durch welche nicht nur die Produktion selbst sondern auch die personenaufwendige Kontrolle tayloristischer Zeitvorgaben weiter automatisiert wurde (Salder und Hachtmann 2009: 176). ${ }^{20}$ "So wie der betriebliche Fordismus das Taylor-System voraussetzt, wurde umgekehrt das fordistische Produktionsregime in der Folgezeit zum Ausgangspunkt für die Entwicklung weiterer Produktionsregime, die an nationale Rahmenbedingungen anknüpften und mit spezifischen technologischen oder auch politischen Entwicklungen verschmolzen« (Hachtmann 2011). ${ }^{21}$ Das Wort Rationalisierung wurde im Diskurs damit weitestgehend durch den Begriff Fordismus ersetzt und auf viele verschiedene Lebensbereiche außerhalb der Fabrik übertragen. »Ihre tendenziell inflationäre Ausweitung verweist auf eine Denkrichtung, die im Glauben an die grundsätzliche Machbarkeit aller Aspekte menschlicher Gesellschaft, deren »Verwissenschaftlichung « forderte und die »Experten« als deren Träger ins Zentrum stellte. Dies bezog sich auf öffentliche Aufgabenfelder wie die Stadtund Raumplanung ${ }^{22}$ ebenso, wie auf Prämissen und Orientierungsmuster einer technokratischen staatlichen und kommunalen Sozial- und Gesundheitspolitik« (Hachtmann 2011[Kursivsetzung Th. M.]). ${ }^{23}$

$=2000 \&$ corpus $=17 \&$ smoothing $=3 \&$ share $=\&$ direct_url=t1 \%3B \%2CFordismus \%3B \%2Cco (zuletzt aufgerufen am 25.02.2018).

20 Henry Ford selbst pries das Fließband mit den Worten an, dass es »strengste Disziplin« erzwinge Online unter: http://docupedia.de/zg/hachtmann_fordismus_v1_de_2011 (zuletzt aufgerufen am 24.02.2018). An dieser Stelle sei angemerkt, dass die teils starke Fokussierung auf die Autobiografie Henry Fords in der wissenschaftlichen Literatur im Grunde selbst einen untersuchungswürdigen Umstand darstellt, da er exemplarisch für die allgemeinen Personalisierungstendenzen in der Geschichtsschreibung technischer und wissenschaftlicher Entwicklungsprozesse ist. Allerdings werden mit diesem Namen dennoch derart viele gesellschaftliche Veränderungen zu einem Komplex zusammengefasst, die sich nicht in einer einfachen Rationalisierungsthese erschöpfen, sodass die schlaglichtartige Beschreibung dieser Entwicklung mit den teils alternativ verwendeten Begriffen Industrialisierung und Industrialismus (und ähnliches gilt bereits für den Taylorismus) weiter verfehlt würden als durch die instrumentelle Vereinfachung Fordismus.

21 Online unter: http://docupedia.de/zg/hachtmann_fordismus_v1_de_2011 (zuletzt aufgerufen am 24.02.2018).

22 So interpretierte der Architekt und Stadtplaner Fred Forbat die Metropole als volkswirtschaftlichen Betrieb neu, der ähnlich funktioniere wie die Fabrik (Hilpert 1995: 141). Auch die Städtebauer Le Corbusier und der Bauhaus-Cründer Walter Gropius, der sich selbst mitunter als »Wohn-Ford « bezeichnete, traten für den Bau von »Wohnmaschinen« ein (Saldern und Hachtmann 2009: 179, Saldern 1991: 168), von denen etwa Corbusiers Modulor - als Versuch in den Wohnungsbau eine am Durchschnittsmaß des menschlichen Körpers ausgerichtete feste mathematische Ordnung zu integrieren - ein aussagekräftiges Zeugnis ablegt. Siehe dazu auch Hilpert (1978).

23 Online unter: http://docupedia.de/zg/hachtmann_fordismus_v1_de_2011 (zuletzt aufgerufen am 24.02.2018). 
Ulrich Bröckling widmet sich hier etwas dezidierter der Frage wie sich die Konzepte der »Lebenssteigerung und Optimierung « zu dieser Zeit gesellschaftlich legitimiert haben und über die Betriebe hinaus operationalisiert wurden (Bröckling 2002: 278). Im Mittelpunkt seiner Analyse steht dabei die genealogische Aufarbeitung der zu Taylors Zeit breit frequentierten Theorie der Menschenökonomie und der in den 1960er Jahren folgenden marktliberalen Humankapitaltheorie, die beide daran beteiligt waren die bedarfsorientierten Menschenbilder der Betriebswissenschaften (zunächst $\mathrm{zu}$ anthropologisieren und schließlich) als Fundament einer staatlichen Bevölkerungspolitik zu etablieren. In beiden Fällen handelt es sich dabei gleichermaßen um eine Aufwertung wie eine Abwertung menschlichen Lebens, das es zwar zu erhalten und zu entwickeln gälte, das im gleichen Zug allerdings radikal ökonomisch als organisches Kapital bzw. Humankapital auf seinen volkswirtschaftlichen Wert reduziert wird. Das Optimum wird auch hier aus dem rein technischen Kontext gelöst, mit Gesundheitsvorstellungen verschränkt und synonym mit einem spezifischen Gleichgewichtsverständnis behandelt, von dem anzunehmen ist, dass es statistisch ermittelt und kontrolliert werden kann. Im ersten Fall, der auf Rudolf Goldscheid zurückgehenden Menschenökonomie, wird eine ausgeglichene Bilanz aus Kosten und Ertrag des »organischen Kapitals« allerdings noch normativ zum Telos planwirtschaftlicher Steuerung erhoben, die sich in der "Qualifizierung des Menschenmaterials « ${ }^{24}$ erfüllen soll. Nach Goldscheid stelle sich dieses $»$ vitale Optimum ${ }^{25}$ vor allem als Lösung des Problems ein

»wie der Mensch durch eine Arbeitszeit von weniger als 24 Stunden sein Leben während 24 Stunden fristen kann, wie er in Stand gesetzt wird, mit einer Arbeit von weniger als 24 Stunden nicht nur die Erhaltung seiner selbst, seiner nicht arbeiten könnenden Kinder und seiner nicht mehr arbeiten könnenden Eltern, sondern auch die Höherentwicklung des Typus Mensch, die Steigerung der Macht des Organischen über die Natur zu bewerkstelligen. ${ }^{26}$

Die Menschenökonomie steht dabei nahezu sinnbildlich für die Generalisierung betriebswissenschaftlicher Prämissen, denen nach die Gesellschaft von der Ebene des Gemeinwesens, über die Familie, bis hin zum Individuum als »Selbsterhaltungsmaschine ${ }^{27}$ konzipiert wird.

Die Standardisierung und sozialtechnische Normierung begannen als spezifische »Mechanik der Macht« (Foucault 1977: 176) die »industrielle Rationalisierung« über die Projektion der modernen Familie in der Folge auch im privaten Alltagsleben zu ergänzen (Bodenschatz 1995: 43), was insbesondere Frauen in der Rolle der 
Haushälterin und Kindererzieherin betraf. In seinen extremsten Ausformungen ging auch dieser Prozess mit der Übertragung von Fabrikstandards in das Design von Küchenmöbeln und die Empfehlung rechnerischer Verfahren wie Zeiterfassung und Haushaltsbilanzen einher. ${ }^{28}$

Die "Leitvorstellung« tayloristisch-fordistischer "Rechenhaftigkeit« (Salder, Hachtmann 2009: 175) und Planungseuphorie war »die gesellschaftliche Maschine orientiert am Ziel des Zerteilens und der Programmierung von Verhalten in allen Bereichen der Gesellschaft « (Hartmann 2016: 139) drang sie nicht nur in die Organisation häuslicher Abläufe vor sondern inspirierte z.B. auch Kunst- und Architekturstile wie den italienischen Futurismus und brachte Unterhaltungsformate wie die rhytmisch-maschinellen Tänze der sog. Tiller-Girls hervor. Durch die vergleichsweise hohen Lohnzahlungen bei gleichzeitiger Massenherstellung von Luxusartikeln wie des Automobils, wurde der Fordismus in weiten Teilen zu einem volkswirtschaftlichen Perpetuum Mobile stilisiert. ${ }^{29}$ Über das Bild der Maschine, installieren sich im Windschatten des gesellschaftlichen Aufschwungs, bzw. einer Verbreiterung der ökonomischen Mittelschicht dabei auch die an Normalität gekoppelten Optimierungsparadigmen in anderen gesellschaftlichen Bereichen. ${ }^{30}$

Im Kontext des zunehmenden Marktliberalismus der Nachkriegszeit setzt sich im globalen Westen zwar die Idee eines staatlich initiierten Wettbewerbs bzw. der Selbstregulation nach Angebot und Nachfrage als universelles Organisationsprinzip gegenüber der staatlichen Kontrolle über die Produktion durch, allerdings weitet auch die nach diesen Grundsätzen ausgerichtete Humankapitaltheorie die Suche nach den Bedingungen der Arbeitsproduktivität potentiell auf alle gesellschaftlichen Bereiche aus. Theodor W. Schutz und Guity Nashat Becker, als weithin prominenteste Vertreter der Humankapitaltheorie, beschwören ab den 1960er Jahren einen zurückhaltenden Staat, der lediglich die gesundheits- und bildungspolitische Entwicklung von Arbeitskräften und Konsument*innen gewährleistet:

28 Besonders interessante (wenn auch extreme) Beispiele stellen etwa die Formulare zur Zeiterfassung von Tätigkeiten im Haushalt dar, die durch die Zeitstudienabteilung des amerikanischen »Bureau of Home Economics« entwickelt wurden. Für eine Abbildung der analogen Zeiterfassungskarten siehe Saldern (2009: 179) oder die durch sie angeführte Originalquelle Witte (1928: 65). Für eine Erläuterung des Designs von (amerikanischen) Küchen unter dem Diktum »Planned to save you time, labor and money«siehe Giedion (1982). Und für eine Originalquelle »The New Housekeeping. Efficiency Studies in Home Management« von Christine Frederick (1922), bei Saldern zitiert nach der deutschen Übersetzung »Die rationelle Haushaltsführung. Betriebswissenschaftliche Studien« von Irene Witte (1922).

29 Vgl. Hachtmann, Rüdiger (2011): Fordismus. Online unter: http://docupedia.de/zg/hachtman n_fordismus_-v1_de_2011

30 In Bezug auf die selbstverstärkende Wirkung dieser Gesellschaftsvorstellungen und ihre subjektivierenden Effekte sei an dieser Stelle nochmals an die Normalismustheorie Jürgen Links erinnert, die unter Abschnitt [X] bereits ausführlicher beschrieben wurde. 
»Bruttoinvestitionen in die Gesundheit bringen Anschaffungs- und Unterhaltungskosten mit sich, einschließlich der Kosten für Kinderbetreuung, Ernährung, Kleidung, Wohnen, ärztliche Dienste und Körperpflege. Die Leistungen, die das Gesundheitskapital abgibt, bestehen aus der »gesunden« oder »krankheitsfreien« Zeit, und sie schlagen sich in der Arbeit, im Konsum und in den Freizeitaktivitäten nieder. Je länger jemand gesund ist, desto länger kann er arbeiten, konsumieren oder Freizeitbeschäftigungen nachgehen. ${ }^{31}$

Während Goldscheid buchhalterische Rationalität mit Humanität gleichsetzt und sein Programm als "normative Wirtschaftswissenschaft « verstanden wissen will, die definiert "wie Menschen ihr gesellschaftliches Zusammenleben ökonomisch gestalten sollen « (Bröckling 2002: 288[Hervorhebung UB]), changiert die Humankapitaltheorie vorgeblich deskriptiv und weitaus näher an der zunehmend populärer werdenden Figur des Homo Oeconomicus »zwischen einer Grammatik der Sorge und einer der Härte« (Bröckling 2002: 291). Der vielstimmige Diskurs des Förderns und Forderns klingt hier bereits leise an, indem etwa eine zu umfassende sozialstaatliche Wohlfahrt als ssoziale Hängematte ${ }^{32}$ und schädlicher Einfluss auf die Entfaltung der natürlichen Nutzenkalküle des Menschen beschrieben wird (Bröckling 2002: 289f.).

Diese wirtschaftswissenschaftlichen Plädoyers für eine größere Eigenständigkeit des Humankapitals (das sich begrifflich über die Arbeitskraft und die Gesundheit nun auch auf Faktoren wie äußeres Erscheinungsbild und Sozialprestige ausweitete), lassen sich bereits als Annahme darüber deuten, dass das idealisierte ökonomische Gleichgewicht in Zukunft vermehrt von der Initiative aller Arbeitenden abhängen wird, da die fordistische Produktionsorganisation im Grunde von Anfang an auf das Scheitern dieses »Wohlstandsbeteiligungsplans« zusteuerte. Denn »die Transportbänder und die mit ihm zunächst lediglich locker verknüpften Maschinen und Apparate verwachsen zunehmend zu einem Komplex teilautomatischer Fertigung zusammen - und drängen schließlich auf Vollautomatisierung« (Hachtmann 2011). ${ }^{33}$

Mit dem Ölpreisschock von 1973, der die Automobilindustrie besonders traf, und der zeitgleich einsetzenden Erodierung der Sozialsysteme verlor der Fordismus als »Gesellschaftsvision« nach der bereits einsetzenden Sättigung der Märkte

31 Schultz (1986[1899]: XII: 15f.) Zitiert nach Bröckling (2002: 289[Hervorhebungen im Original]).

32 Becker (1998): 118f). Zitiert nach Bröckling (2002: 291).

33 Online unter: http://docupedia.de/zg/hachtmann_fordismus_v1_de_2011 (zuletzt aufgerufen am 24.02.2018). Hachtmann zitiert das Wort »Wohlstandsbeteiligungsplan« frei nach der Autobiografie von Henry Ford 1956[1923]). 
und Produktivitätssteigerungen im Zusammenhang mit der massenhaften Entlassung von Angestellten wirtschafts- und sozialpolitisch weiter an Glaubwürdigkeit. Parallel wurden die Heim- und Herd-Paradigmen der fordistischen Gesellschaft und die mentalitätsgeschichtliche Geltung, mit der der Fordismus die Kulturproduktion regierte, vermehrt durch die sozialen Bewegungen in Zweifel gezogen und herausgefordert. Das Bild der gesellschaftlichen Maschine mit seinen deterministischen Implikationen, also die für dieses Konzept weitestgehend elementare Fremdbestimmung im Produktionsprozes, durch Handlungsprogrammierung und Negierung von Individualität, verliert spätestens zu diesem Zeitpunkt gänzlich seine Popularität als gesellschaftliches Selbstbild. Die gesellschaftliche Entwicklung widerlegt damit bisweilen dystopische Prognosen denen nach diese Wirtschaftsordnung die »heute den Lebensstil aller Einzelnen, die in dies Triebwerk hineingeboren werden - nicht nur der direkt ökonomisch Erwerbstätigen -, mit überwältigendem Zwang bestimmt « und auch weiterhin »bestimmen wird, bis der letzte Zentner fossilen Brennstoffs verglüht ist « (Weber 1973[1934]: 379[sic! Hervorhebung im Original]). Und im gleichen Maße, in dem sich die Prämissen der Ökonomie auf andere gesellschaftliche Bereiche ausweiten, gleitet der Ökonomietheorie die Bestimmung der Arbeitskraft als ursprünglich maschinistisches »Komplement einer auf Triebkraft von Verbrennungsmotoren basierenden industriellen Produktion « (Herrmann 2002: 193f.) sukzessive aus den Händen.

\section{3 Über den »bebenden Sockel der Kräfteverhältnisse « ${ }^{34}$ - Zur Ambivalenz des Begriffs der Arbeitskraft}

Auch Foucault, der ebenfalls nie einen Zweifel daran gelassen hat, dass sich die Entfaltung dessen, was er als Biomacht ${ }^{35}$ beschreibt seit dem 18. Jahrhundert »koextensiv« und immer stärker im Zusammenhang mit der Geschichte des modernen Kapitalismus vollzogen hat (vgl. Brieler 2007: 249), fasst das Interesse, das die Arbeit an den Potentialen des Lebens entwickelt, mit dem Ausdruck der Kräfte.

Obgleich die Konzeptionen einer »Ökonomie der Bio-Macht « und die Beschreibung der prozeduralen Herausbildung der Bevölkerung als (vorrangig statistisches) Interventionsfeld »der Regierungstechniken« (2000[1978]: 64) nicht immer konsistent miteinander verbunden sind (vgl. Bröckling 2002: 175f.), bleibt seine Konzeption allerdings auch unter den Bedingungen einer weitestgehend postindustriellen,

\footnotetext{
34 (Foucault 1983[1976]: 114).

35 In der Reihe »Sexualität und Wahrheit« verwendet Foucault die Begriffe »Biomacht« und »Biopolitik«nahezu synonym (vgl. Brieler 2007: 249). Sein Begriff des »Bios« ist dabei antiessentialistisch und verweist als »Biomacht« auf seine Historizität. Leben ist ein soziales Konstrukt und Effekt historischer Wissenspraxen (vgl. Brieler 2007: 252).
} 
modernen Informationsökonomie (die eben längst Daten zu ihrem neuen Öl erklärt hat) weiter anschlussfähig.

So schreibt Foucault etwa, dass es der Biomacht, unter deren Diktum sich »Akkumulation der Menschen und Akkumulation des Kapitals« (Foucault 1977: 83) ${ }^{36}$ parallel vollziehen und die »das Leben verwaltet und bewirtschaftet « (Foucault 1977: 166) um es »in einem Bereich von Wert und Nutzen zu organisieren « (Foucault 1983[1976]: 171), vor allem um eine »Steigerung der kollektiven und individuellen Kräfte« (Foucault 1983[1976]: 37) ) $^{37}$ geht.

Der bestimmende ökonomietheoretische Fokus des historischen Materialismus und auch vieler sozialtheoretischer Beschreibungen, die in ihrer Begriffsbildung wesentlich dem Vokabular der angewandten Betriebswissenschaften folgten, wird hier aber erweitert indem etwa der relativ eng auf physische Entitäten zugeschnittene Begriff der Arbeitskraft für eine subjekttheoretische Konzeption geöffnet wird. ${ }^{38}$

Umgekehrt ließe sich sagen, Foucault entwickelt das Konzept der Biomacht zwar »auf dem Niveau der ökonomischen Prozesse und der sie tragenden Kräfte« (Foucault 1983[1976]: 168), setzt den Begriff aber nicht mit Produktivität, Leistungsvermögen und ihrer Erhaltung gleich.

Werden die Arbeiter*innen in der kritischen Ökonomietheorie, reduziert auf die durch sie individuell abrufbare Arbeitskraft, auch als solche bezeichnet (Diestelhorst 2016: 46f.) zielt der Begriff der Kräfte nach dem produktiven Machtverständnis Foucaults (und im Zusammenhang mit der Regierung des Lebens) genau zwischen eine biopolitische Reduzierung auf den Körper (bzw. der ihm eigenen

36 Zitiert nach Brieler (2007: 249)

37 Zitiert nach Brieler (2007: 249, 250).

38 Die nicht gleichbedeutend mit dem Konzept der Persönlichkeit ist und auch in seiner Quantität nicht an die Ebene des Individuums gebunden ist: So geht er vielmehr explizit davon aus »daß die politische Ökonomie sich von dem Moment an hatte bilden können, als unter den verschiedenen Elementen des Reichtums ein neues Subjekt aufgetaucht war, nämlich die Bevölkerung« (Foucault 2004[1977-1978]: 159[sic!]). Eine Dimension des Begriffs, die Bröckling, ohne dass eine weitere Reflexion erfolgt, in den Begriff des Kollektivsubjekts auslagert. Lässt die konzeptionelle Flexibilität des Subjektbegriffs bei Foucault noch deutlich die sprachtheoretischen Wurzeln durchscheinen, bleibt er in Bröcklings Zeitdiagnostig scheinbar für eine Bedeutung reserviert, die in ihrer letzten Konsequenz (wenn schon nicht echte Menschen, wie bei Reckwitz) so doch individuelle Selbstwahrnehmungen meint, die sich ggf. überindividuell ähneln. Da Bröckling diese Seite der Neukonzeption konsequent durchhält, gewinnt er gegenüber der Foucault'schen Variante deutlich an Kontur. Der Begriff des Kollektivsubjekts ist (ohne weitere Erläuterung) dagegen etwas problematisch, da er vor allem eine bewusste Selbstidentifikation als Hauptmerkmal des durch ihn beschriebenen Zusammenhangs nahelegt, der aber nicht näher eingegrenzt ist und damit viele konkurrierende Formen von Kollektivität (politische, funktionale, nationale, ethnische usw.) nebeneinander stehen lässt. 
Fähigkeiten) und eine Beschreibung sozialer Entitäten, dessen Deutung und Verständnis sich als Ensemble von Kräften - oder besser Kräfteverhältnissen - herausbildet. Es geht hier nicht allein um das Mitdenken der Psyche (so wie es schon die Humankapitaltheorie selbst tut) und auch nicht um die im Grunde triviale Aussage, dass das menschliche Leben nicht gleichbedeutend mit seiner Arbeitskraft ist, sondern um »die Vielfältigkeit von Kräfteverhältnissen, die ein Gebiet bevölkern und organisieren; [um] das Spiel, das in unaufhörlichen Kämpfen und Auseinandersetzungen dieses Kräfteverhältnis verwandelt, verstärkt und verkehrt (Foucault 1983[1976]: 114]). Es geht also vielmehr um die Entwicklung eines Konzeptes, demnach die determinierenden Menschenbilder der Ökonomie mit widerständigen Selbstbeschreibungen ein Kräfteverhältnis bilden, das ohne die jeweils andere Seite unvollständig wäre. Die Arbeitenden erscheinen als Subjekt nicht nur abhängig von der sie beschreibenden Instanz oder den sie bestimmenden materiellen Verhältnissen, sondern als Gleichzeitigkeit kontrahierender Fremd- und Selbstverständnisse.

So lässt sich gerade die »Geburt der Biopolitik« (Foucault 2004[1977-1978]) als umfassende Genealogie einer Form von Humankapitalisierung lesen, die sich über die (vielfach auch bei anderen Autor"innen) beschriebene Gleichmachung von Körpern und ihre sozialstaatliche Steuerung hinausgehend, vermehrt auf die Subjektivität ausrichtet (Brieler 2007: 250). Diese Genealogie zielt damit konkret, auf die Beschreibung der interdiskursiven Beziehungen verschiedener Liberalismus- und Neoliberalismus-Formen, als Elemente einer Regierungskunst, die unter den Bedingungen von Freiheit zwar auf die Ökonomisierung und Regulierung des Selbst ausgerichtet ist, dieses Ziel aber niemals völlig erreicht, an Widerständigkeiten scheitert und dabei einen Gutteil seiner produktiven Macht gerade aus der fortlaufenden Einhegungen dieser Independenzen bezieht. Die durch Foucault Ende der 1970er Jahre entstehenden historischen Analysen zur Synthese von Subjekt und Wirtschaft entwickeln sich an diesem Punkt zu einer bis heute gültigen Gegenwartsdiagnose.

Vor allem in den Analysen der an Foucault anschließenden Gouvernementalitätstheorie (aber auch der Arbeits- und Industriesoziologie) wurden in den letzten Jahrzehnten Konzepte wie Disziplin und Unterdrückung entsprechend als primäre Kennzeichen moderner Arbeitsverhältnisse durch (scheinbar) gegenteilige Begriffe wie Flexibilität und Improvisationsfähigkeit ersetzt (vgl. Pongratz und Voß 2003). Die prekären Existenzen in modernen Arbeitsfeldern werden vielmehr durch Eigeninitiative charakterisiert und zeichnen sich gerade durch den Mangel an Vorstrukturierung von Abläufen und erwartbaren Regelmäßigkeiten aus. ${ }^{39}$ Wohinge- 
gen die Disziplin lediglich gelehrige Körper »fabriziert « (Foucault 1977: 176), verlagert sich mit dem Produktionsort der modernen Wissensökonomie auch das produktive Moment der Macht zunehmend ins Subjekt selbst. Mit Blick auf die Gestaltung moderner Arbeitsprozesse bedeutet dies, dass sich ein Gutteil der Gestaltungsmöglichkeiten nun zwar auch in den Verantwortungsbereich des Individuums erstrecken, das Subjekt dadurch jedoch zum Ursprung und Ziel einer entinstitutionalisierten Disziplin wird, die als verlängerter Arm die kollektive Normierung im (oder man könnte auch sagen als) Subjekt reproduziert. Die schrittweise Entsprechung avantgardistischer Freiheitsrufe vollzieht sich dabei institutionell, juridisch und materiell mit der gleichzeitigen Überführung von erkämpfter Individualität und künstlerischen Selbstverwirklichungsmöglichkeiten in Output- und Gleitzeit-Steuerung, bzw. in eine erneuerte Ökonomie der unmittelbaren Marktkonkurrenz, die seit den 1980er Jahren vor allem unter den Begriffen »Postfordismus« und »Postmoderne« firmiert.

schen Aufschwungs, inzwischen Zombiefilme produziert werden, greift die These eines klar markierbaren Übergangs von Industrieproduktion zu Dienstleistungsgesellschaft zu kurz und wurde entsprechend auch vielfach als vereinfachend und ahistorisch kritisiert. Zudem wurde ihr eine europäisch oder westlich verengte Sichtweise unterstellt, da entsprechende Arbeitsformen massenhaft in sog. Schwellen- oder Entwicklungsländer exportiert wurden. Auch in der aktuellen Debatte um Datenarbeit oder sog. Clickwork zeigt sich deutlich, dass sich fordistische Produktionsstrukturenim Zeichen der Digitalisierung auf den Bereich der modernen Informationsökonomie übertragen oder dort spezifische Mischvarianten ausbilden. So zählt es zu den besonderen Charakteristiken von algorithmischen Arbeitswelten, dass sie die fordistische Atomisierung von Arbeitsvorgängen im Grunde noch verstärkt, sie dabei aber gleichzeitig unsichtbar macht. Dies z.B. durch enthusiastische Fortschrittsrhetoriken und technische Systeme wie Webmasken oder Apps-, die sich zwischen die Arbeitenden, Kund“innen und Produzent"innen schalten. So lässt sich etwa Amazons Plattform »mechanical turk «als eine Inkarnation fordistischer Industriearbeitsstandards im informationstechnischen Cewand ansehen, die es sog. Requestern erlaubt menschliche Intelligenz so zu drenagieren, dass sie damit in der Summe Aufgaben erledigen können, zu denen Computer derzeit nicht in der Lage sind (oder für die Computer schlicht zu teuer sind). Kommerzielle Webservices können so z. B. das durch Menschen vorgenommene massenhafte »taggen «von Fotos oder Überprüfen von Kaufvorgängen anhand spezieller APIs einbinden, sodass oberflächlich kein Hinweis darauf besteht, wie das System die anfallenden Aufgaben löst. Fast überall wo von »künstlicher Intelligenz « gesprochen wird, ist irgendwo die Arbeit solcher Clickworker eingegangen. Amazon selbst spricht hier von »artificial, artificial intelligence. « Quelle: https: //www.mturk.com/ (zuletzt aufgerufen am 01.11.2017). Gleichzeitig reproduziert sich auch die Cenealogie der militärischen Betriebsführung in der Logistik-Sparte des Konzerns. In einer Stellenausschreibung für ein sog. Fullfillment-Center schreibt Amazon: »Die Einstellung von Personal aus dem Militär ist ein entscheidender Bestandteil unseres unternehmensweiten Business-Plans, die Führungskräfte der Zukunft zu finden. «Quelle - »Amazon.de Military Specialist Talentpool 2018 « https://www.amazon.jobs/en/jobs/595164/de-military-specialist -talentpool-2018-fur-fachkrafte-aus-dem-bereich-logistik-transport-der-bundeswehr (zuletzt aufgerufen am 18.02.2018). 
Von Sennet (2000) über Hardt und Negri (2002), Boltanski und Chiapello (2003) bis hin zu Reckwitz (2011) herrscht weitestgehend Einigkeit darüber, dass insbesondere das Kräftezerren der sozialen Bewegungen seit 1968 als Geburtshelfer der hierzu passenden neuen Subjektivität anzusehen ist. Demnach ist es nicht die Technisierung oder Computerisierung moderner Arbeitsprozesse allein, die das Subjekt unter Anpassungsdruck setzt; vielmehr speist sich die Veränderung der Arbeitsprozesse aus der Widerständigkeit der Produktivkräfte und ihrer erneuten institutionellen Einhegung. Die subjektgeschichtliche Bedeutung der späten 1960er Jahre liegt (aus dieser Perspektive) demnach insbesondere in der Entstehung von Gegensubjektivitäten, die zunächst unvereinbar mit den systemischen Erfordernissen des Fordismus sind, hier mittelfristig aber zu einer Reorganisation festgefahrener Arbeitsstrukturen und der Entstehung neuer Märkte führen und damit auch zum Stimulus neuer Steuerungsstrategien werden (Brieler 2007: 254). Die verschlungenen Wege über die sich diese Gegensätze im zeitlichen Verlauf immer wieder kreuzen, sollen hier nicht im Einzelnen Nachgezeichnet werden, stattdessen liegt der Fokus der folgenden Abschnitte vielmehr auf den Aspekten dieses Zusammenhangs, die in besonders deutlicher Weise mit spezifischen Eigenheiten des Self-Tracking-Diskurses verbunden sind. Hierbei ist vor allem an die konzeptuelle Differenz zwischen den Forderungen der sozialen Bewegungen und den sozialpädagogischen Selbsthilfeprogrammen zu denken, die sich begrifflich in der Differenz zwischen Emanzipation und Empowerment widerspiegelt.

Wo die (z.B. medizin- und reproduktionstechnisch zugespitzten) Emanzipationsbestrebungen kollektiver Zusammenschlüsse wie z.B. der feministischen Bewegung aber auch der Anti-Psychiatrie- oder der Krüppelbewegung auf eine Politisierung gesellschaftlicher Strukturprinzipien abheben (vgl. Abschnitt IV.), versammeln sich hinter der Empowermentrhetorik in der Summe vor allem verschiedene Regierungsprinzipien, die auf dem programmatischen Fördern und Fordern von Selbstverantwortung und Eigenständigkeit basieren und die Subjektivität selbst als dominierende Kraft (Brieler 2007: 244) einer Ökonomie erschließen, die zunehmend auf den relativen Freiheiten der Individuen basiert. Auch die Quantified-Self-Community drängt auf eine aktivere Rolle in der Gestaltung sozialer, politischer und ökonomischer Prozesse, sieht von einer grundsätzlichen Hinterfragungen ihrer Organisationsbedingungen allerdings ab. Durch sie werden zwar verschiedene Formate zur Erhöhung von Selbstbestimmung getestet, die Prinzipien durch die die gewonnene Autonomie zugleich nutzbar gemacht wird, bleiben jedoch unangetastet. Entsprechend findet der Begriff der Emanzipation im Diskurs des Self-Trackings auch kaum Verwendung, während der Begriff des Empowerment dagegen sehr präsent ist und im Verbund mit einer revolutionären Innovationssemantik sogar wesentlich ihr Selbstbild als soziale Bewegung bestimmt. Um die spezifischen Autonomievorstellungen der Quantified-Self-Community bzw. des lose mit ihr verbundenen Self-Trackings 
allgemein und die Problemszenarien und Zielrichtungen der zugehörigen Technologien genauer konturieren zu können, erfolgt im folgenden Abschnitt ein Rekurs auf einige gouvernementalitätsteoretische Auseinandersetzungen mit dem Konzept des Empowerment und der Funktion die es vorallem in der fordistischen Strukturkrise für die Transformation und Stabilisierung der sozialen und ökonomischen Gesellschaftsorganisation einnahm.

\section{4 »In praise of a paradox « ${ }^{40}$ - Der Freiheitszwang des Empowerment}

Wie Bröckling konstatiert, ist die Verwendung des Empowerment-Begriffs, spätestens seit der US-Amerikanische Psychologe Julian Rappaport seinen Aufstieg in den grundlegenden Begriffsapparat der Sozial- und Gesundheitspolitik einläutete, als ein Sammelbegriff anzusehen, der seine Karriere vor allem der oberflächlichen und alltagstauglichen Verschmelzung äußerst disparater Konzepte verdankt (vgl. Bröckling 2007: 184). Empowerment kann über unterschiedlichste soziale und politische Fraktionierungen, Milieus oder fachliche Disziplingrenzen Plausibilität für sich beanspruchen und scheint eine Art universelle Anziehungskraft auszustrahlen (vgl. Cruikshank 1999), die in seiner kurzen Genealogie - angefangen bei sozialen Bewegungen bis hin zu biopolitischen Agenturen - kaum einen Bereich unberührt gelassen hat, in dem sich mit dem Verhältnis von Selbst- und Fremdbestimmung auseinandersetzt wurde.

Wie Bröckling betont, fällt eine präzise Bestimmung des Begriffs deshalb so schwer, da er sowohl eine deskriptive wie auch eine präskriptive Seite enthält. Empowerment ist gleichzeitig Ziel, Mittel, Prozess und Ergebnis persönlicher und sozialer Veränderungen. So bezeichnet der Terminus sowohl eine Wertorientierung, an der sich die Handlungen Einzelner oder die von Gruppen und Communities ausrichten können und sollen, als auch ein theoretisches Modell um die dazu notwendigen Transformationen auf individueller, organisatorischer oder gesellschaftlicher Ebene zu definieren. Zudem ist ihm in entkontextualisierter Form nicht anzusehen, ob er in Form seiner transitiven oder reflexiven Bedeutung verwendet wird; ob er also als ein professionelles, meist verhaltenspsychologisches Konzept verwendet wird das die eigene »Lebensbewältigung " fördert aber auch fordert, oder ein meist politisches und den emanzipativen Bewegungen der späten 6oer Jahre nahestehendes Konzept meint (vgl. Bröckling 2007: 180f.). Obgleich die Praxis beider Ansätze zunächst nahezu identisch sein kann (in einem ersten Schritt z.B. auf die Ausstattung einzelner Individuen mit bestimmten Fähigkeiten ausgerichtet ist), verwehrt sich die Selbstermächtigung im Kontext der politischen Formeln

40 Mit »In Praise of a Paradox« betitelte Julian Rappaport, Namensgeber des Empowermentkonzepts, sein Plädoyer für diese neue Form einer Sozialpolitik (1981). 
sozialer Bewegungen einer gleichzeitigen Internalisierung von Problemursachen und kritisiert vielmehr die gesellschaftliche Bedingtheit individuell auftretender Probleme oder die individualisierende Kraft von Problembildern als solche. ${ }^{41}$ Hierbei ist z.B. an die Feministinnen des Boston Women's Health Book Collective, das später auch als Our Bodies, Ourselves Collective in Erscheinung trat. Vor allem in den Jahren zwischen 1966 und 1971 richtete das Kollektiv Workshops und Konferenzen aus, bot Vernetzungsinfrastruktur und gab eigene Publikationen heraus. Zusammengenommen dienten die Aktivitäten dazu Frauen einen Zugang zu ihrem Körper zu ermöglichen, der vom medizinischen Blick des patriarchalen Paternalismus unabhängig war. Neben konkreten Selbstermächtigungspraxen auf der individuellen Ebene (etwa der Vermeidung ungewollter Schwangerschaften oder der Bewältigung gesellschaftlich weithin unsichtbar gemachter Probleme wie z.B. der postnataler Depressionen) zielte ihre Kritik auf die Art und Weise in der der Frauenkörper im medizinischen Diskurs hergestellt und beherrscht wird. ${ }^{42}$ Auch die nach dem Vorbild der Frauenbewegung gegründeten sog. Krüppelgruppen, diente neben konkreten (Selbst)hilfestellungen der kollektiven Diskursintervention, um das Thema Behinderungen aus der Verfügung medizinischer Definitionen zu lösen und breiter als gesellschaftlich bedingte Probleme zu diskutieren. Auch diese Form politischer Zusammenschlüsse dient zwar der Stärkung individueller Möglichkeiten und Rechte, setzt sich letztlich allerdings gerade die Endindividualisierung von Problemen zum Ziel (Mürner und Sierck 2009). Befreiung von Bevormundung und die Überwindung von Marginalisierung basiert demnach »auf der Konstruktion eines Bewusstseins, dass als phantasievolles Erkennen der Unterdrückung neue Handlungsmöglichkeiten eröffnet « (Harraway 1995: 34), die sich letztlich gegen diese Zustände als Ganzes richten und die nicht - wie die sozialpädagogischen Empowermentprogramme - bei ihrer Kompensation auf individueller Ebene stehen bleiben.

Eine besonders richtungsweisende Auseinandersetzung mit dem Konzept des Empowerment findet sich bei der feministischen Gouvernementalitätstheoretikerin $^{43}$ Barbara Cruikshank, die mit »The Will to Empower - democratic citizens and

41 So z.B. im Konzept des Ableismus, dass die Abwertung und Ausgrenzung von Menschen mit Behinderungen in allgemeiner Weise als Bestandteil des Konzeptes Behinderung problematisiert.

42 Siehe dazu vor allem die bewegungsinterne Schriftensammlung »Unser Körper - unser Leben« (Blume et al. 1980).

43 Gouvernementalität in der bei ihr verwendeten Weise geht als das Ergebnis zahlreicher Weiterentwicklungen und Übertragungen auf die Beschreibung ganz verschiedener Machtformen dabei bereits deutlich über das hinaus, was Foucault in der Ceschichte der Couvernementalität im Sinn hatte: »Mit diesem Wort »Gouvernementalität« möchte ich drei Dinge sagen. Ich verstehe unter »Couvernementalität« die aus den Institutionen, den Vorgängen, Analysen und Reflexionen, den Berechnungen und den Taktiken gebildete Cesamtheit, wel- 
other subjects« (1999) die "politische Logik« und die »Ökonomie« des Empowerment (1999: 70ff.) als Antwort auf die Krise der Regierbarkeit gegen Ende des 20. Jahrhunderts beschreibt. ${ }^{44}$

Obgleich die jeweiligen Begründungen, Intentionen und Ziele die mit dem Begriff verbunden werden also stark variieren, je nachdem in welchem Kontext er angewendet wird, liegt in der Unklarheit vermutlich auch einer der Gründe für die rasante Karriere des Begriffs. Obgleich seine Implikationen wie Selbstständigkeit und Unabhängigkeit zeitgenössische Werte darstellen, die Spektren-, Milieu- und Lagerübergreifend geteilt werden, bleibt er meist unspezifisch genug um die genaue Ausformung der Zielparameter offen zu lassen und sich so zur Zielfläche der Projektionen aller an Empowerment-Prozessen beteiligten Akteure zu machen. Der Begriff des Empowerment ist insofern weniger ein Vehikel um unter dem Anschein des Entgegenkommens zu bewirken, dass im Zuge der Selbstermächtigung auch eine Selbstüberzeugung sozialer Bewegungen mit den politischen Zielen biopolitischer Agenturen bewirkt wird, sondern vielmehr eine Art neutrale Brücke zwischen verschiedenen, politischen Territorien, die ihre Stabilität aus einem bereits bestehenden, übergeordneten Konsens bezieht, der in der Erlangung größtmöglicher Selbstbestimmung liegt. Der Begriff hat vor allem deshalb früh das Interesse der Gouvernementalitätsforschung auf sich gezogen, da er ein sehr deutliches Beispiel dafür darstellt, wie im Kräfteverhältnis von Ermächtigungsansprüchen und ihrer partiellen Erfüllung somit »Theorie und Technologie der Führung zur Selbstführung verschmilzen « (Bröckling 2007: 184). ${ }^{45}$ Wie Nicholas Rose schreibt, hat sich der Wunsch nach Freiheit durch seine Unhintergehbarkeit an dieser Stelle gleichsam zu einer neuen Form der Vermachtung entwickelt, die nicht nur weitaus besser zu den Erfordernissen einer modernen Ökonomie und ihrer politischen Regulation passt, sondern, anders als die Disziplinarmacht, auch wenig Angriffsfläche bietet. Denn Freiheit kann als nicht umfangreich genug kritisiert werden, aber es ist

che es erlaubt, diese recht spezifische, wenn auch sehr komplexe Form der Macht auszuüben, die als Hauptzielscheibe die Bevölkerung, als wichtigste Wissensform die politische Ökonomie und als wesentliches Instrument die Sicherheitsdispositive hat« (Foucault 2004[19771978]: 162).

44 Die Diagnose einer Krise der Regierbarkeit ist vermutlich an Foucaults Aussagen zu einer »Krise der Regierung« orientiert, die er im Cespräch mit Ducio Trombadori erläutert (Foucault 2005b[1980-1988]: 51ff.). Foucault bezieht sich hier allerdings auf die Blockade der »Regierungskunst« des 17. Jahrhunderts. Ihm geht es hier darum zu sagen, dass die bisherigen Technologien der Menschenführung zu dieser Zeit ihre transzendentale und übergeschichtliche Gültigkeit verlieren. Zu den Herrschaftsformen der Souveränität und der Disziplinierung treten fortan noch die Steuerungsprinzipien der Biopolitik, mit der das Subjekt Bevölkerung einhergeht (Foucault 2004[1977-1978]: 158).

$45 \gg$ In short, the question of governance becomes a question of self-governance in the discourse of self-esteem« (Cruikshank 1993: 328). 
schwer eine grundsätzliche Ablehnung gegen jene Programme und Maßnahmen $\mathrm{zu}$ formulieren, die im Zeichen der Freiheit auftreten: »The norm of self-esteem links subjectivity to power; it "binds subjects to a subjection that is the more profound because it appears to emanate from our autonomous quest for ourselves, it appears as a matter of our freedom « (Rose 1990: 256 [Hervorhebung im Original]). ${ }^{46}$ Mit dem Begriff Self-Esteem benennt er zudem einen Aspekt von Empowerment, der als eine Art reflexiver Teilaspekt des Empowerment noch weitaus weniger auf der Erlangung oder Erweiterung tatsächlicher Macht im z.B. rechtlichen Sinne (vgl. Bröckling 2007: 205) basiert und vielmehr auf die Produktion eines reflexives Selbstverhältnisses ausgerichtet ist, das intrinsische Potentiale zur Verbesserung der eigenen (und im Zuge dessen auch der gemeinschaftlichen) Lage freisetzen soll.

Barbara Cruikshank untersucht und theoretisiert diesen speziellen, psychologisierenden Empowerment-Modus anhand einer Auseinandersetzung mit der sog. Self-Esteem-Bewegung, die im Kalifornien der 1980er Jahre angetreten ist, um soziale Probleme qua Selbstwertsteigerung der Betroffenen zu bekämpfen.

Diese Studie, die in der Tradition gouvernementalitätstheoretischer Argumentationsmuster die Prinzipien, die Foucault in seinen Analysen herausgearbeitet hat, auf die Höhe der Zeit hebt, macht deutlich, dass diese Bewegung mitunter quer zu einer eindirektionalen Steuerungsbeziehung zwischen der institutionellen Politik und einem durch sie klassifizierten Milieu liegt. D.h. obwohl klassische Professionen auch hier eine wichtige Rolle in der Konzeption, Repräsentation und vor allem der Initiation entsprechender Projekte und Maßnahmen spielen, zeichnet sich unter den avisierten Bevölkerungsteilen, $\mathrm{zu}$ denen sich die Initiator*innen mitunter selbst zählen, dabei diskursiv gleichermaßen eine hohe Identifikation mit den initiierten Projekten ab:

"Government and experts cannot fix these problems for us. It is only when each of us recognizes our individual personal and social responsibility to be part of the solution that we also realize higher $>$ self-esteem « $^{47}$

Dies verdeutlicht genau das Spezifikum dieser modernen Formen von Steuerung, die sich im Nachgang der emanzipativen Bewegungen zwischen Begriffen wie Selbstorganisation, Improvisation, Autodidaktik und einem durchgängigen Argwohn gegenüber Autoritäten der Meinungsbildung, Planung und Entscheidung oder der Konsumtion von Massenprodukten aufgespannt haben (vgl. Bröckling

46 Zitiert Nach Cruikshank (1993: 331).

47 Appendix des Berichtes »Toward a State of Esteem: The Final Report«, der California Task Force to Promote Self-Esteem and Personal and Social Responsibility. Herausgegeben vom California Department of Education (1990: vii). Zitiert nach Cruikshank (1993: 328). 
2007: 185ff.), dabei allerdings gelernt haben auf die Eigenständigkeit sozialer Umwälzungsprozesse zu spekulieren. Es ist insofern ein Konzept, dass sehr stark auf der Assoziation einer positiven Zukunft basiert, deren Erreichbarkeit sie durch den Glauben an sich selbst in Aussicht stellt. "Self-esteem is above all a metaphor, a symbol filled with excess meaning that can ignite visions of what we as a people might become« (Cruikshank 1993: 332).

Empowerment in seiner allgemeinsten Formel - als Erweiterung der Möglichkeiten über das eigene Leben zu bestimmen (Bröckling 2007: 181) - wird im Zusammenhang mit Self-Esteem dabei noch stärker als in anderen Kontexten in kausaler Beziehung zur Willenskraft konzeptionalisiert.

Ferner entwickelt es sich dicht am Konzept der Chancengleichheit, als zentrale Legitimationsformel der neoliberalen Marktwirtschaft und wird auch auf die Selbstbestimmung innerhalb dieser Rahmenbedingungen begrenzt. Es stellt einen eigentümlichen Hybrid dar, der sowohl die transitive als auch die reflexive Bedeutungsweise des Begriffs referiert, d.h. er erscheint als professionelle Selbstanleitung eigenverantwortlichen Alltagsmanagements, die im Sinne einer politischen Strategie als Ermächtigung wahrgenommen wird. Genauer, als eine Ermächtigung darüber den Anrufungen der Gegenwart unter Anwendung eigener Methoden nachzukommen.

Entsprechend setzt ein derartiger Ansatz auch weniger auf der Ebene von Strukturproblemen an und verlagert im Zuge der Skizzierung eines Subjekts, das seine Bedingungen durch bloße Willenskraft verändern kann, mittelfristig auch die Ursache für soziale Probleme in den Verantwortungsbereich der Individuen. Der Self-Esteem-Ansatz verkehrt seinen instrumentellen Freiheitsbegriff damit nicht nur zur Pflicht, sondern tritt leicht ersichtlich als ein Subjektivierungsprogramm an, »that will solve social problems from crime and poverty to gender inequality by waging a social revolution, not against capitalism, racism and inequality, but against the order of the self and the way we govern our selves" (Cruikshank 1993: 329).

Aus gouvernementalitätstheoretischer Sicht stellt der Ansatz einen administrativen Apparat dar, der in seiner Beziehung zur Bevölkerung »zugleich ein Apparat des Wissens ist (Foucault 2004[1977-1978]: 397), da er das Subjekt sukzessive von der Notwenigkeit der Überwindung eines Zustandes überzeugt, der durch seine immanenten Perspektiven, seine Begrifflichkeiten, Kontexte und das Wissen auf das er rekurriert, überhaupt erst definiert wird. Der Self-Esteem-Ansatzes bringt dabei paradoxer Weise das Subjekt hervor, dass er bekämpft.

Dabei handelt es sich um einen Prozess an dem die universitären Disziplinen wie die Psychologie mit ihren verschiedenen Bindestrich-Sparten ebenso beteiligt sind wie die Pädagogik oder die sozialwissenschaftliche Forschung und die theoretischen aber insbesondere empirischen Werkzeuge, die sie Kommissionen und Ämtern für die Identifikation von Handlungsbereichen und die Plausibilisierung 
ihrer Maßnahmen überantworten (Cruikshank 1993: 332). Auch hierbei ist natürlich in erster Linie an quantifizierende und klassifizierende Verfahren zu denken, die den Prozess der Selbstwertsteigerung und die damit verbundenen Freiheitsbegriffe durch einen Rationalitätskontext erden. In Bezug auf Ian Hacking (1986) schreibt Cruikshank: "Self-esteem is a practical and productive technology available for the production of certain kinds of selves, for »making up people«, [...] Selfesteem is a technology in the sense that it is a specialized knowledge of how to esteem our selves, to estimate, calculate, measure, evaluate, discipline, and to judge our selves. It is especially, though not exclusively, a literary technology« (Cruikshank 1993: 329[Hervorhebung im Original]). Zahlen nehmen also auch Cruikshank zur Folge einen wesentlichen Stellenwert ein, den sie 1999 nochmals als »Ruling by Number « zusammenfasst (1999: 104ff.) und der verbunden mit der Technologie des Self-Esteem letztlich jene Verschmelzung aus Selbst- und Fremdführung bewirkt, die potentiell gesellschaftsweit Subjektivierungsprogramme über ihre designierten sozialen Problemfelder entfaltet: »A whole society of esteemed, estimated, quantified and measured individuals can replace a citizenry defined by their lack of selfesteem« (Cruikshank 1993: 334).

\section{Gründe dich selbst - Empowerment und "Kalifornische Ideologie"}

Obgleich keine Kausalbeziehung unterstellt werden soll, wäre es irrig die mustergültigen Prinzipien des Self-Esteem als Bestandteil der »Kalifornischen Ideo$\operatorname{logie}{ }^{48} \mathrm{zu}$ leugnen. Umgekehrt rekurriert auch der Self-Esteem-Ansatz auf die regionaltypischen Fortschrittserzählungen Kaliforniens, das den EmpowermentBegriff hervorgebracht hat (Bröckling 2007: 185; 189).

»self-esteem is a kind of »liberation therapy« that requires a complete reorientation to social problem-solving, as well as the mobilization of an effort compared by advocates to landing on the moon, the discovery of the atom, and it calls for the mobilization of »every Californian «. $\ll^{49}$

Die Mobilisierung aller Kalifornier schließt die kreativen Wissensarbeiter*innen der Technologiemetropolen in San Francisco mit ein. In seiner appellativen Funktion richtet sich das Konzept des Self-Esteem überdies auch an Personen, bei denen gerade keine offensichtlich korrekturbedürftigen Probleme bestehen. Die wissenschaftlich angeleitete Optimierung der (lange Zeit maschinell übersetzten) Beziehung zwischen Ökonomie und Mensch, wird mit der gesellschaftlichen Extension ökonomischer Prinzipien so zu einer Optimierung des Menschen selbst. Die Idee es

48 (vgl. Barbrook und Cameron 1995).

49 Appendix des Berichtes »Toward a State of Esteem: The Final Report«, der California Task Force to Promote Self-Esteem and Personal and Social Responsibility. Herausgegeben vom California Department of Education (1990: vii). Zitiert nach Cruikshank (1993: 328). 
könnte eine anthropologische Konstante geben, der nach der Mensch ein von Natur aus optimierendes Wesen sei (vgl. Straub et al. 2012: 40f.), hat ihren Ursprung möglicher Weise in solchen potentiell gesellschaftsweiten Strategien der Eindämmung, Dekonstruktion und Beseitigung von Wachstumsstillstand oder mangelndem Veränderungswillen als diskursfähige Positionen.

Dies wird nicht zuletzt auch durch die diversen Belege darüber plausibilisiert, dass Prekarisierung, die Gefahr des finanziellen und sozialen Abstiegs, sowie die hiermit verbundenen Ängste bei weitem keine Phänomene sind, die nur außerhalb der (akademischen) Wissensökonomien im Zusammenhang mit geringqualifizierter Erwerbsarbeit bestehen. Ganz im Gegenteil wird das Silicon Valley häufig als nahezu sinnbildlich für eine prosperierende wirtschaftliche Ballung führender Technologiebranchen betrachtet, die sich essentiell auf eine strukturelle Prekarisierung auf hohem Niveau (Manske 2007) stützt (vgl. Abschnitt III. 2.2). Entsprechend liegen kurzfristiger ökonomischer Erfolg und sozialer Abstieg hier immer nah beieinander. Self-Esteem funktioniert nicht nur in die Aufstiegsrichtung sondern kann auch in der entgegengesetzten Weise auf die Angst vor zukünftigen Abstiegen einwirken, die Ursache in der Vokabel des Scheiterns individualisieren, nur um sie dann in einem erneuten Richtungswechsel zur Chance zu erklären.

Mehr noch als in anderen Regionen der Welt hat sich die auffordernde Ermutigung zu mehr Selbstvertrauen und Eigeninitiative hier im zeitlichen Verlauf immer weiter mit einem marktradikalen Liberalismus vermengt, der sich selbst nicht zuletzt dadurch unhinterfragbar macht, dass er jeden Zweifel auf das innere Verhältnis zurück spiegelt, das das Subjekt zu sich selbst unterhält: »Today, a state of esteem can be founded upon the inner dialogue between self and self « (Cruikshank 1993: 333). Damit sind auch die Wurzeln der Gründerszene mit dem Diskurs der methodischen Selbstgründung verschränkt.

Wohingegen das Empowerment der sozialen Bewegungen seinen Teil zur Krise der fordistischen Produktion beitrug, überantwortet das Empowerment im Dienste gouvernementaler Regierungsprogramme den Arbeitenden die Suche nach der Lösung der im Zuge postfordistischer Produktion auftretenden Strukturprobleme.

Eines der hervorstechendsten Strukturprobleme bildet die für die Organisation der Produktion wichtige Bemessung von Arbeitsvorgängen in der immateriellen Ökonomie der Zeichen (Reckwitz 2011: 141). Wohingegen das Set an Qualifikationen, das von einer Facharbeiterin erwartet wird, durch die eindeutigen Mechanismen der zugehörigen Verwertungsprozesse klar eingegrenzt wird, existieren in Bezug auf selbstständige Kreativarbeit kaum derartige Normen, die ein Verhältnis zwischen den Arbeitsprozessen und ihren Produkten definieren. Dieses Problem potenziert sich je weiter sich die Produktionsorte in das Selbst verlagern bzw. je stärker sich Arbeitsvermögen und Persönlichkeit zu kongruenten Kategorien entwickeln. 
Dabei handelt es sich um einen Umstand der in direkter Folge auch die Bemessung der Leistung affiziert, da sie immer weniger im Rahmen der relativen Abgeschlossenheit bürokratischer Angestelltenverhältnisse oder disziplinarischer Maschinenparks evaluiert, analysiert und gesteigert werden kann. Die Erodierung von Normalarbeitsverhältnis und des zugehörigen Konzeptes »Arbeitsplatz« hinterlässt hier zu aller erst ein Orientierungsproblem, für das zunächst weder der moderne Staat noch die wirtschaftsnahen Wissenschaften eine Lösung anbieten.

Trotz der zunehmenden Probleme Arbeitsvorgänge über den Leistungsbegriff adäquat zu erschließen, wird aber nicht automatisch sein Ende eingeläutet - im Gegenteil, die postindustrielle Gesellschaft ist nahezu »besessen von Leistung, und sie scheint umso besessener je weniger es objektive Bewertungskriterien für sie gibt« (Klopotek und Scheiffele 2016: 36). Denn eine konstante Optimierung der Leistung wird durch den sich erhöhenden Wettbewerbsdruck vieler kleiner unabhängiger Unternehmen und Entrepreneure für das Bestehen in der Konkurrenz im Grunde immer dringender. Wenn also das Scientific Management und seine institutionellen Erben keinen Fuß in die Tür der Ich-AG bekommen, können Zeitmanagement sowie körperliche und psychische Leistungsfähigkeit der kreativarbeitenden Freelancer daher von niemand anderem mehr optimiert werden als von ihnen selbst.

\subsection{Selbstoptimierung oder »die Zeit anders leben « ${ }^{50}$}

Das hervorstechendste Merkmal der Selbstoptimierung ist demnach, dass sie die konstitutive Differenzierung zwischen Subjekt (optimierende Instanz) und Objekt (Gegenstand der Optimierung) aufhebt (Straub et al. 2012:30). ${ }^{51}$ Selbst etwas zu optimieren, heißt im Zusammenhang mit diesem Begriff also immer sich selbst zu optimieren. Die Gouvernementalitätstheorie thematisiert Selbstoptimierung (schon im Zusammenhang mit ihren kritischen Analysen der Ratgeberliteratur) als zeitgenössische und allgegenwärtige Pflicht, die in der modernen Gesellschaft an die Stelle des Gehorsamszwangs getreten ist und unterschiedliche Formen des Einwirkens und der Animation zu einem Modus der Subjektivierung vereint, der Individuen frei zu setzen vermag, ihnen aber gleichzeitig Sachzwänge und Verantwortung für sich selbst aufbürdet (vgl. Bröckling 2003: 339). Ein Zusammenhang zwischen Selbstoptimierung und einem zweischneidigen Freiheitsverständnis wird dabei meist vorausgesetzt und z.B. als Grundlage wirtschafts- und sozialpolitischer Programme, wie der Agenda 2010 ausbuchstabiert, die in der bereits beschriebenen

50 Foucault, Michel (2003b[1976-1979]).

51 Zitiert nach Röcke (2017: 2). Siehe dazu auch Link (2012). 
Weise als Mischverhältnis aus Expertokratie, Empowermentpädagogik und methodischer Menschenführung (Bröckling 2007: 191) ein komplementäres Subjekt für die moderne Arbeitswelt zu erzeugen versuchen: „Die Freiheit vom Disziplinarzwang wird erkauft mit der Pflicht zur permanenten Optimierung und Selbstoptimierung. Die Macht die Empowerment verspricht und verleiht, haben die Bemächtigten auf sich selbst zu wenden, und diese subjektivierende Faltung soll sie produktiver machen, als äußere Autoritäten es jemals vermögen« (Bröckling 2007: 212). Allerdings bleiben die Gouvernementalitätsstudien auch in der Auseinandersetzung mit der Beziehung die das Empowermentkonzept zu dem der Selbstoptimierung unterhält häufig bei ihrer Problematisierung als Regierungsinstrumente stehen, die sich vergleichsweise einseitig nur als intentionaler Einwirkungsversuch auf die Entstehung einer Subjektivität darstellen. ${ }^{52}$ Das empirische Material der Gouvernementalitätsanalysen (die Manuale, Richtlinien, Empfehlungen und Paragraphen, die zusammengenommen das durch sie beschriebene Programm bilden) sind zwar meist dermaßen deutlich formuliert, dass an ihren Absichten kein Zweifel besteht, für die Beschreibung einer diskursiven Formation oder eine Abschätzung über die gesellschaftliche Wirkmächtigkeit dieser Programme fehlt es hier allerdings an diskursiver Resonanz - z.B. in Form anderer Materialtypen, Schauplätze und Nebenstränge. Kurzgefasst: Eine Subjektivität wie das unternehmerische Selbst erscheint so gerade nicht als Kräfteverhältnis, sondern lediglich als kritisch kontextualisierte Imagination biopolitischer Agenturen. Dies entspricht vollends dem Anspruch der Gouvernementalitätsstudien und der selbstgewählten Materialbegrenzung mit der sie operieren, führt allerdings auch dazu, dass sie bei der Beschreibung der produktiven Komponente der Macht so zwangsläufig auf der Ebene normativer Appelle verhaftet bleibt und kaum mehr als das geeignete Mittel erscheint um eine spezielle Form der Produktivität zu beschreiben, die sich explizit aus dem Verhältnis von Macht und Gegenmacht entwickelt hat.

In Bezug auf die eigentümliche Art in der Selbstoptimierung und Empowerment sich innerhalb der Selbstvermessung verschränken, lässt sich vom Standpunkt einer heterogen ausgerichteten Diskursanalyse dagegen genauer sagen, dass es sich bei der Erfindung von numerischen Verfahren, in Teilen um einen Ansatz zur Lösung von Problemen handelt, die aus der relativen Freisetzung der Individuen erst erwachsen. Die Quantified-Self-Bewegung wendet sich gegen die staatlichen Agenturen und ihre administrativen Monopole und doch redet sie ihren Programmen das Wort. Sie ist weit mehr als eine Selbsthilfegruppe für sozialpsychologisches Empowerment und ihre Experimente liegen teils quer zu den Doktrinen der Gouvernementalität. Denn es gab im Jahr 2007 augenscheinlich keine Agentur die verlangte: »Quantifizier dich selbst!« Dennoch hat die Subjektivität die sie 
stützt die kongruenten Aktivierungsimperative längst internalisiert. Ihr Empowerment geht folglich auch nicht weiter als bis zur punktuellen Problematisierung jenes Fundaments, das für die unabhängige Selbstentfaltung im Rahmen zeitgenössischer Ökonomieprinzipien unentbehrlich ist: Der ökonomischen und biopolitischen Datenlage.

In einer Bestandsaufnahme verschiedener Self-Tracking-Projekte, die Gary Wolf 2010 für das New York Times Magazine vornahm und die als eine der am stärksten frequentierten Selbstbeschreibungen der frühen Jahre von Quantified Self gilt, tritt vor allem die Infragestellung der Allgemeingültigkeit offizieller Wissensreservoires als zentrales Element hervor. In Bezug auf den Computerspezialisten Bo Adler schreibt Wolf:

»[the] idea that we can - and should - defend ourselves against the imposed generalities of official knowledge is typical of pioneering self-trackers. $\ll^{53}$

In dieser kurzen Zeile sind drei zentrale Implikationen enthalten, welche die Außenwirkung und das Selbstverständnis der Quantified-Self-Community in den frühen Jahren prägen und auch eine hohe Bedeutung für die Selbststilisierung zur Bewegung einnehmen: 1. Die Positionierung gegenüber offiziellem Wissen, dessen Entstehungsbedingungen den meisten Menschen intransparent sind, 2. das aus diesem Grund als Gefahr wahrgenommen wird 3. und gegen das sich die Pioniere der Selbstvermessung zur »Wehr« setzen.

Dieses Zitat stellt zudem nur eines unter vielen Beispielen dafür dar, dass die frühe Phase diskursiver Identitätsgebung der Quantified-Self-Bewegung in hohem Maße auf der Artikulation einer grundsätzlichen Skepsis gegenüber gesellschaftlichen Wissensbeständen basiert, die sich darüber hinaus nicht in der Infragestellung einzelner Datenaggregate erschöpft, sondern die Bedeutung institutioneller Wissensproduktion und -verwaltung für das Zustandekommen eines gesellschaftlichen Allgemeinwissens und damit auch der eigenen Weltzugewandheit hinterfragt.

»The self-tracker is empowered to make this refusal not just by information, however, but by quantified self-knowledge. $\ll^{54}$

Wenn sich Cruikshank also auf Foucault beruft um die subjektivierende Kraft jener "politischen Technologien" (Foucault 1993[1988]: 146) zu beschreiben, die das Individuum an die Imagination einer Gesamtgesellschaft oder sozialen Einheit wie

53 Quelle: www.nytimes.com/2010/05/02/magazine/o2self-measurement-t.html?_r=2\&ref=mag azine\&page-wan-ted=all (zuletzt aufgerufen am 02.09.2015).

54 Quelle: „Empowerment Through Numbers? Biomedicalization 2.0 and the Quantified Self« http://thesociety-pages.org/cyborgology/2012/09/06/empowerment-through-numbersbiomedicalization-2-0-and-the-quantified-self/ (zuletzt aufgerufen am 18.02.2018). 
einer Nation oder eines Staates rückbindet und dabei auch an die statistischen Evaluationen und ihre medialen Repräsentationen denkt (Cruikshank 1993: 330), argumentiert sie auf der Höhe eines technologischen Standes, der sich seither selbst überholt hat.

Denn numerische Verfahren werden durch die Quantified-Self-Community dazu genutzt, um unter den Vorzeichen des Empowerment und in der Tradition seiner Ansprüche und Ziele gerade eine Herauslösung des Individuums aus dem statistisch manifestierten Gesellschaftskörper zu ermöglichen. Kongruent zu den Postulaten der Selbstwertsteigerung nehmen die Beiträge zum gemeinschaftlichen Wohl hier den Umweg über die eigene Person. Anders als durch die gouvernementalen Kritiken beschrieben, verschiebt sich der Konnex zwischen statistischen Verfahren, Individualität und Gemeinschaft, im Zuge der Selbstquantifizierung dabei aber von der Rezeption kollektiver Vergleiche zur unabhängigen Herausforderung dieser Vergleichsbasis.

Wobei diese Herausforderung lediglich die Aussagekraft dieser Daten und nicht die definitorische Macht von Daten per se betrifft. Das Quantifizierte Selbst sieht sich in den Aggregaten der Bevölkerungsstatistik zwar nicht genügend repräsentiert, strebt ansonsten allerdings vor allem danach, die gleichen Praktiken und Wissensformen anhand der formalistischen Methodik des Self-Trackings auf sich selbst anzuwenden.

Die Quantified-Self-Community folgt keinem ausformulierten Programm. Sie folgt weder den Ratschlägen einer Kommission noch einer Task Force. Dennoch übertrifft sie sich von Jahreskonferenz zu Jahreskonferenz mit der Menge an neuen Zielen und den mit ihnen koinzidierenden Verfahren. Bei der Quantified-SelfCommunity ist keine Hilfe zur Selbsthilfe in der gouvernementalen Auslegung konventioneller Motivationskonzepte oder ähnlicher Anrufungen zu erkennen, jedenfalls keine die in direkter Weise an sie gerichtet würde. Stattdessen folgen die SelfTracker*innen vielmehr einer Selbsthilfe zur Selbsthilfe. Diese folgt einem handlungspraktischen Empowerment-Verständnis, das sich im Tun verwirklicht und keine anderen politischen Appelle als jene nach mehr Möglichkeiten der Selbstkontrolle kennt. Diese Bewegung muss durch niemanden aktiviert oder im Sinne Bröcklings "ge-nudget ${ }^{55}$ werden, denn sie hat die strukturellen Parallelen zwischen der antiautoritären Auflehnung nach innen und die neoliberale Indienstnahme des Individuums als Schmied des eigenen Glücks bereits internalisiert und zu einem Teil von sich gemacht. Das quantifizierte Selbst »stupst« sich selber an und dies längst nicht mehr nur zur Steigerung des eigenen Selbstvertrauens, der eigenen Fähigkeiten, Marktchancen, Kreditwürdigkeit und Lebenslage, sondern zur Optimierung der Optimierung des Selbst.

55 Siehe dazu: Bröckling (2015). Online unter: https://soziopolis.de/beobachten/kultur/artikel/g esteigerte-tauglichkeit-vertiefte-unterwerfung/). 
Das quantifizierte Selbst geht über die drängenden Empfehlungen hinaus, gesunde Nahrung zu konsumieren, die Kalorien zu zählen, richtig zu schlafen und genügend Sport zu machen. Es protokolliert und korreliert so lange bis es herausgefunden hat was unter Maßgabe der individuellen Besonderheiten seines individuellen biologischen Metabolismus, seiner Lebenssituation, den täglichen Umwelteinflüssen oder den psychischen und genetischen Prädispositionen genau gemeint ist, wenn allgemein von gesunder Nahrung, richtigem Schlaf und genügend Sport gesprochen wird.

Als praktische Kritik richtet es sich gegen den Widerspruch des allgegenwärtigen Ansporns zu mehr Selbstbewusstsein im Zeichen der Individualisierung von Risiken und Verantwortung bei gleichzeitigem Ausbleiben einer Individualisierung der gesundheitlichen und wirtschaftlichen Bemessungsgrundlagen. Die gleichen Agenturen, die das Individuum zu mehr Selbstkontrolle animieren, können selbst nur über den Durchschnitt der Bevölkerung informieren. Diese statistischen Großkategorien werden in den meisten Fällen natürlich methodisch in Kohorten, Berufsgruppen, Krankheitsbilder oder andere Kategorien aufgespalten; das Wissen das sie anbieten beziehen die Agenturen allerdings dennoch aus einem Vergleich, der die individuelle Dimension allgemeiner Probleme und vor allem die eigenen Leistungen zum Verschwinden bringt. Die Agenturen fördern so die herausragende Individualität ihrer Klient*innen, Patient*innen und Angestellten und drängen sie doch immer auf den Durchschnitt zurück.

Hier wird nicht mehr nur durch Manifeste und Bürgerforen auf die Politik eingewirkt (vgl. Abschnitt III. 2f.), sondern es werden grundsätzlich die Regierungsmittel und ihr Wissen in Frage gestellt. Es wird nicht mehr appellativ versucht die Aufmerksamkeit, der offiziellen Statistiken auf die durch sie vergessenen Bereiche $\mathrm{zu}$ lenken, sondern es wird eine allgemeine Bemächtigung der Datenhoheit angestrebt um eine adäquate Selbstregierungsgrundlage zu schaffen.

Durch die Entwicklung und Anwendung von individuellen Messverfahren bringen die Self-Tracker*innen dabei allerdings performativ das hervor, was sie semantisch negieren - einen biopolitischen Machtzusammenhang: Im Zuge der systematischen Beobachtung vergangener oder gegenwärtiger Körper- und Geisteszustände ist das Subjekt zwar einerseits Souverän über eigene Technologien, Datenerhebungen und -deutung, allerdings transportiert es dabei übergeordnete biopolitische Prämissen in den Mikrokosmos seiner individuellen Lebenswelt: »Ist der Datenkörper der Bevölkerung Teil einer historisch vertrauten, präventiven Politik des Risikomanagements, so repräsentiert sich hier ein personalisierter Datenkörper als Ort mikroskopischen Selbstmanagements« (Wiedemann 2016a: 65).

Im gouvernementalitätstheoretischen Doppelsinn der Selbstkontrolle installiert sich so die einzige Kontrollform, die für die Gewährleistung und Steigerung der Produktivität im Selbst-Unternehmen sinnvoll ist. 
»David has managed to create a streamlined workflow allowing him to record everything from sleep, weight and food intake to productivity, yoga and meditation. [...] He says his dashboard has done »wonders to keep me accountable, and more importantly, to help me notice when I have fallen off the horse, allowing me to keep on track with my goals. « For those of us (all of us) looking to optimize our workflows and understand our habits, this is definitely a talk to see. ${ }^{56}$

Der Intensitätsgrad mit dem dieses Subjekt seine Selbstdefinition von der ökonomischen Lebensorganisation abhängig macht, zeigt sich einerseits schon in der Selbstverständlichkeit mit der hier Produktivität und essentielle Bedürfnissen wie Schlaf und Nahrungsaufnahme verwoben werden, andererseits aber auch darin, dass im Zuge des Self-Tracking sogar Schlafen als Kompetenz erscheint über die man die Kontrolle verlieren kann (Wiedemann 2016a: 89) - wenn das Subjekt zum Produktionsort wird, wird die Optimierung der Produktion zur Selbstoptimierung.

\subsection{Disziplin und Selbstdisziplin}

Obgleich mit der zunehmenden Verbreitung digitaler Produktionstechnologien »letztlich alles quantifizierbar gemacht werden kann« und sich auch für die Qualität von Arbeitsergebnissen unterschiedlichste "messbare Indikatoren festlegen « lassen (Bröckling 2014: 17), stellt sich die Frage danach, welche Indikatoren die richtigen sind und wie sie zu jenen der Arbeitsleistung in Beziehung stehen als nicht minder komplex dar, und zu ihrer Beantwortung scheint kaum verallgemeinerbares Wissen zur Verfügung zu stehen. Bei der Aufgabe die richtigen Daten $\mathrm{zu}$ erheben, sie gewinnbringend zu korrelieren und so das optimale Arrangement zwischen Arbeitsmodus und Produktqualität auszutarieren bleiben die selbstständig arbeitenden Individuen scheinbar in so vielen Fällen sich selbst überlassen, dass die Produktivitätsanalyse als strukturelles Problem der postfordistischen Arbeitswelt im Allgemeinen erscheint.

Das Paradox, dass im Zuge voranschreitender Liberalisierung die Bemessung und der Nachweis von Leistung gleichermaßen schwieriger und wichtiger wird, verspricht sich in der Entwicklung zugehöriger Introspektionstechniken zumindest vorübergehend aufzulösen. Vorübergehend deshalb, da eine dem Leistungserhalt und der Leistungssteigerung dienende Optimierung aller hierfür relevanten

56 Quelle: »Dashboard of My Life« Teaser für einen Vortrag des Self-Trackes David de Souza auf der Quantified Self Conference 2017. http://quantifiedself.com/2017/05/qs17-preview-dashbo ard-life/ (Link zuletzt aufgerufen am 17.01.2018). 
Aspekte, anders als in der hierarchisch organisierten Arbeitswelt, auch nicht länger an einer vordefinierten Zielvorgabe ausgerichtet ist und sich daher unentwegt verschiebt.

Dennoch erschöpft sich die Beschreibung dieses entscheidenden Wandels nicht in einer einfachen Entgrenzungsthese. Das durch die Massenmedien vielfach beschworene Bild der numerischen Optimierung ist insofern korrekturbedürftig, als dass Optimierung nicht immer gleichbedeutend mit Steigerung ist, sondern, ganz ähnlich wie in den tayloristischen Zeitstudien, nicht selten die Suche nach dem höchstmöglichen Leistungsniveau meint, dass über längere Zeit eingehalten werden kann (vgl. Abschnitt III. 6.3). Auch Bröcklings vergleichsweise pauschaler Optimierungskritik kann daher unter den spezifischen Voraussetzungen des SelfTracking-Diskurses nur bedingt zugestimmt werden, obgleich das Self-Tracking die Akribie und Selbststrenge vieler anderer Optimierungspraxen sicher übertrifft: »Das Optimum selbst ist hier zwar nicht vorgegeben, wohl aber die Richtung, in der es angestrebt wird. Der Vektor wird ins Unendliche verlängert. Theoretisch ist grenzenlose Verbesserung möglich. Programme der Steigerung tendieren theoretisch zur wissenschaftlichen Rationalisierung im Sinne des one best way und praktisch zu Strategien der Disziplinierung und Selbstdisziplinierung« (Bröckling 2014: 17).

So werden basale Prinzipien der disziplinarischen Arbeitsorganisation nicht zuletzt auch im Rückbezug auf die wissenschaftlichen Standards der Industrieproduktion in den Diskurs des Self-Tracking importiert, obgleich sie mit einer etwas unentschiedenen Ambivalenz aus Kontinuität und Bruch diskutiert werden. Wohingegen die (vor allem kalifornische) Rahmenerzählung des modernen Arbeitsethos sich aus der bewussten Abgrenzung mit den tayloristisch/fordistischen Produktionsprinzipien speist, sind die Self-Tracking Projekte in Bezug auf ihre Effizienzfokussierung zuweilen strukturell ähnlich ausgerichtet, entwickeln tayloristische Prinzipien weiter und artikulieren gelegentlich eine kritisch untersetzte Faszination. Argumentativ wird dieser Widerspruch z.B. durch die selektive Referenz auf utopistische Pioniere des Scientific Managements, wie die Taylor-Schülerin, Zeitmessungs- und Bewegungsforscherin Lillian Moller Gilbreth geglättet. Ansätze für das moderne Arbeitsverständnis der Creative Class werden auf diese Weise schon in den Produktivitätsstudien der 1920er gesucht. So schreibt Gary Wolf in einer Rezension einer aktuellen Biografie Gilbreths:

»[...] she'd taken what began as Taylorist dogma and turned it into a practice of close observation and participatory learning that almost turned it on it's head. Instead of seeing human beings as a factor of production, to be exploited like any other resource until worn and replaced, she asked about the human factor in pro- 
duction: what was work for, what were its conditions and benefits, and how could it be improved. $\ll^{57}$

Wird dieses Verhältnis umgedreht, lassen sich also auch in modernen Arbeitsformen, die diskursiv in der Semantik persönlicher Sinnstiftung und Persönlichkeitsentfaltung erscheinen, die strukturellen Ähnlichkeiten zum Taylorismus besser als kontinuierliche Fortschrittsgeschichte beschreiben, im Zuge derer sich die Produktionsoptimierung auf dem Höhepunkt ihrer Strukturkrise lediglich der asymmetrischen Kontrollprinzipien entledigt hat. Die richtigen Prinzipien werden dieser Lesart nach in der Folge aus ihrer falschen Verwendung befreit, müssen aber auch sogleich angewendet werden, um die systemischen Probleme zu bereinigen, die durch die hierarchische Arbeitsorganisation hinterlassen wurden:

»Automation and routinization works best under controlled circumstance, but controls fail, and someone has to clean up the mess. ${ }^{58}$

Eine Besonderheit der Quantified-Self-Community besteht demgemäß auch darin, dass sie (z.B. vom Standpunkt gouvernementaler Empowermentprogramme aus betrachtet) sowohl das Maß an Selbstkontrolle, als auch ihre enthusiastische Artikulation als Weg der Befreiung noch steigert. Sie erscheint als Effekt und Organisationsort produktiver Macht, das die graduellen Freiheiten der Selbstverantwortung mit neuen Anwendungsformen alter Disziplinartechniken verbindet.

Während mit der Zurichtung an der Maschine gebrochen wird, wird Gilbreth zaghaft als Pionierin einer Form von Produktivitätsmessung schraffiert, die heute unter eigener Aufsicht im Home Office stattfindet. Seit den Anfängen der Communitybasierten Selbstvermessung ist dabei in engem Zusammenhang mit Quantified Self eine ganze Reihe an numerischen Productivity-Tracking-Tools entstanden. So z.B. die umfangreiche Software RescueTime:

»This week its Rescue Time. This service sits on your machine and tracks everything you do. If you install the browser extension, it tracks every website you visit. They then do some crowdsourced categorisation of your activities to inform your productivity. $\ll^{59}$

Die selbstreferentielle Anwendung von Zahlen, so wie sie durch RescueTime angeboten wird, soll es den Nutzer*innen ermöglichen, sich durch die Aufdeckung von

57 Gary Wolf, Quelle: http://quantifiedself.com/2017/03/making-time-lillian-moller-gilbreth (zuletzt aufgerufen am 04.01.2018[Hervorhebung durch Gary Wolf]).

58 Gary Wolf, Quelle: http://quantifiedself.com/2017/03/making-time-lillian-moller-gilbreth (zuletzt aufgerufen am 04.01.2018[Hervorhebung durch Gary Wolf]).

59 Quelle: http://tableautim.com/tabtqs-week-1-rescue-time/ (zuletzt aufgerufen am 04.01. 2018). 
Regelmäßigkeiten von vermeintlich falschen Gewohnheiten $\mathrm{zu}$ >emanzipieren dem die täglichen Routinen und Verhaltensmuster zunächst als numerische Muster sichtbar gemacht werden. Als Orientierungsfolie dienen Balkendiagramme, Tabellen und Durchschnitte, die durch längerfristige Nutzung erstellt werden und die Nutzer*innen mit den Werten vergangener Tage, Wochen oder Monate konfrontieren. Die Parameter lassen sich dabei frei modifizieren und den eigenen Arbeitskontexten anpassen; zudem können auch Ziele gsetzt oder Restriktionen verhängt werden, wie z.B. das Sperren bestimmter Internetseiten. Daten, die nicht automatisch durch die Software erfasst werden können (wie z.B. die Mittagspause oder Meetings), lassen sich unter Zuhilfenahme der entsprechenden App nachtragen, die sich anschließend selbstständig mit den übrigen Software-Komponenten auf PCs und Tabletts synchronisiert.

Moderne Selbstverdatungssysteme wie RescueTime, können als Versuche aufgefasst werden, in eine geringfügig vorstrukturierte Arbeitskultur routinemäßige Abläufe und generalisierbare Bewertungskriterien wieder einzuführen. An diesem Beispiel wird geradezu deutlich, dass die Uhr als etwas, dass Foucault in Überwachen und Strafen zur Verdeutlichung der disziplinarischen Normen von Einschließungsverhältnissen (wie der modernen Fabrik) herangezogen hat, hier bereits als etwas anderes erscheint. Die Uhr, die als simple Mechanik eine Konvention prozessiert und es ermöglicht Work von Recreation zu trennen, lässt sich auf das komplexe Gewirr aus Arbeits- und Freizeitintervallen, das nicht selten den Arbeitsalltag der Kreativökonomie bestimmt, nicht in gleicher Weise anwenden. Vielmehr müssen zunächst unterschiedliche qualitative und quantitative Parameter bestimmt, erfasst und in Beziehung gesetzt werden, um die unterschiedlichen Schattierungen vieler Arbeitsformen zu durchdringen. Ein Vorgang der auch durch das mehrfache Starten und Stoppen der Arbeitszeit nicht mehr zu gewährleisten ist. Vielmehr wird versucht, durch den Einsatz von komplexer Software das Quantum und die Qualität der Arbeitszeit aus einem entgrenzten Freizeit-Arbeit-Gemisch herauszudestillieren, das sich nicht mehr in der Binarität Arbeit/Nicht-Arbeit ausdrücken lässt. Der Datenhunger dieser Systeme ist symptomatisch für den potentiell grenzenlosen Inklusionsanspruch postfordistischer Arbeitsparadigmen - da sie mit einer zeitgenössischen, dauerhaft prekarisierten Subjektivität koinzidieren die sich selbst nicht mehr von ihrer Arbeit zu unterscheiden weiß und deren Social-MediaAktivitäten daher immer gleichzeitig der Pflege von privaten und beruflichen Kontakten dient.

Der offensichtlichen Inkonsistenz, dass diese Applikationen eine auffällige Nähe zu den Zeiterfassungssystemen in Großraumbüros oder eben der peniblen Erfassung einzelner Arbeitsschritte im Kontext des Scientific Managements aufweisen bzw. als ihre logische Weiterentwicklung erscheinen, wird allerdings vorausgegriffen, indem der rationalistischen Geschichte der Zeitmessung (nicht zuletzt 
unter Berufung auf Pionierbiografien wie die von Gilbreth) eine zweite emanzipative Lesart gegenübergestellt wird.

In den für das avantgardistische Selbstverständnis der Quantified-SelfCommunity typischen Extremformen der Selbstverrechnung wird dabei nicht selten das kleine Einmaleins der Startup-Plattitüden zu der Semantik des SelfGrowth addiert:

»I've been hacking RescueTime, which tracks how I use my computer, to make a tool for personal growth rather than work optimization. $\ll^{60}$

In einem Experiment koppelte der Self-Tracker Kyrill Potapov seine Arbeitsleistung, gemessen am Produktivitäts-Output der Software RescueTime, an die Versorgung seiner Zimmerpflanze mit dem (über)lebenswichtigen Licht einer Glühbirne und spekuliert dabei auf die erbauende Wirkung der direkten Überführung von ideeller Leistung in materielles, haptisches und beständiges Wachstum, das als symbolisch aufgeladener Indikator der eigenen Vitalität nicht nur das Werk sondern die ganze Person in der Blüte stehen lässt. ${ }^{61}$ Zusätzlich synchronisierte er die durch RescueTime grundsätzlich vorgenommene Unterscheidung zwischen "productive time« und »distracting time« mit einer persönlichen Zufriedenheitsbewertung einzelner Tätigkeiten.

»When he spends time on things he finds personally fulfilling, like working on his PhD, the light turns on and the plant grows. [...] The arrangement adds a new dimension to his productivity data. Every couple of days, Kyrill opens the box to water the plant. This ritual provides an opportunity to take stock on how he has been using his time, based on the condition of the plant. «" ${ }^{62}$

Diese organisch-technische Verschaltung lässt sich zum einen natürlich als ein Spiel mit Begriffen wie Green Economy und dem Chic von Ökologie- und Nachhaltigkeitsphilosophie lesen, die als ethische Begleitmusik während der letzten Jahre

60 Aus einem Teaser für einen Talk des Self-Trackers Kyrill Potapov während der Quantified Self European Conference 2017 in Amsterdam. Quelle: http://quantifiedself.com/2017/05/qs17-pr eview-externalizing-health-rewards/ (zuletzt aufgerufen am 10.11.2017).

61 »Reflecting on his own mostly computer-based work, Kyrill noted how little of a physical trail he leaves in the world. Could his time and productivity data leave a mark on anything? [...] Kyrill explored this idea by connecting the time-tracking service RescueTime to a light placed in a box with a house plant[...]. «Aus der Dokumentation eines früheren Vortrags von Potapov mit dem Titel: »Tracking Productivity for Personal Growth. «Die Pflanze wurde ohne Angabe von Gründen zwischenzeitlich ausgetauscht. Quelle: http://quantifiedself.com/2017 /09/qs17-highlight-kyrill-potapov-tracking-productivity-personal-growth/ (zuletzt aufgerufen am 10.11.2017).

62 Quelle: http://quantifiedself.com/2017/09/qs17-highlight-kyrill-potapov-tracking-productivity -personal-growth/ (zuletzt aufgerufen am 10.11.2017). 
zunehmend die Selbstdarstellung der New Economy bestimmt; zum anderen verdeutlicht dieses Experiment aber auch, dass es sich bei dieser Beziehung um keine harmonische Symbiose von Naturprinzipien, sondern um ein einseitiges Abhängigkeitsverhältnis handelt, dass in den periodisch zu erwartenden Konjunkturtiefen der Leistungsfähigkeit nicht nur das Wachstum hemmt, sondern sogar zu einem Entzug der Lebensgrundlage führt. Regression herrscht hier nicht erst dann, wenn keine Arbeitsanstrengungen mehr unternommen werden, sondern bereits wenn »im Dunkeln« bleibt »welche Faktoren in eine Quantifizierung« der aufgewendeten Anstrengungen einfließen sollen (Diestelhorst 2016: 42).

Wo sich die Produktivität, bzw. die Leistungsfähigkeit aus der Zufriedenheit mit der eigenen Arbeit bemisst und umgekehrt, bedeutet die Unwissenheit darüber wie diese Zufriedenheit herzustellen ist bereits Stillstand und Zerfall der Lebensbedingungen.

»When he's caught up in other activities, the leaves yellow and die. ${ }^{63}$

Ganz ähnlich wie die digitale Rankenpflanze auf dem Pixeldisplay von einem der ersten Fitbit-Gürtel-Clips, muss auch die Zimmerpflanze jeden Leistungseinbruch mit dem Verlust von Blättern büßen, von denen jedes einzelne ein Zeugnis unzureichender Balance in der Lebensführung ist. Die Tatsache sich mit Arbeitsvorgängen $\mathrm{zu}$ beschäftigen, die nicht das eigene Wohlbefinden fördern oder der Persönlichkeitsentwicklung dienlich sind, treten hier als persönliches Versagen in Erscheinung das mögliche Auswirkungen auf das Umfeld immer gleich mit aufzeigt. ${ }^{64}$ Die Zwangsmomente, die der Self-Growth-Semantik eingepflanzt sind, kommen hier ungeniert zum Vorschein:

$»[$...] tying the health of another to one's own behavior can be an extremely motivating force. ${ }^{65}$

Das Hacking von Technologien als immanenter Bestandteil der Innovationspraxis der Community - das auch vor Technologien keinen Halt macht die, wie RescueTime oder FitBit, selbst durch Mitglieder der Quantified-Self-Community entwi-

63 Quelle: http://quantifiedself.com/2017/09/qs17-highlight-kyrill-potapov-tracking-productivity -personal-growth/ (zuletzt aufgerufen am 10.11.2017).

64 So werden die gelben und welken Blätter der Pflanze zu einer Chronik der Unausgeglichenheit. »A record of my failures right there among all the green leaves. «Quelle: http://quantifie dself.com/2017/09/qs17-highlight-kyrill-potapov-tracking-productivity-personal-growth/ (zuletzt aufgerufen am 10.11.2017).

65 Quelle: http://quantifiedself.com/2017/05/qs17-preview-externalizing-health-rewards/ (zuletzt aufgerufen am 10.11.2017). »Distractions take on a new threat. Rather than just endangering his goals, they now threaten the health of Eddie [der Pflanze. Anm. Th.M.].« Quelle: http://quantifiedself.com/2017/09/qs17-highlight-kyrill-potapov-tracking-productivity -personal-growth/ (zuletzt aufgerufen am 10.11.2017). 
ckelt wurden - ist im Diskurs allerdings meist mit einer abgemilderten Bedeutung des Begriffs belegt, der eher die Umnutzung oder Kombination verschiedener Technologien und Dienste als z.B. die technologieaktivistische Sensibilisierung einer breiteren Öffentlichkeit für Sicherheitslücken o.ä. bedeutet. Diese Form von Umnutzung führt kongruent zu den Selbsterzählungen der Quantified-SelfCommunity zu einem frühen Post von Gary Wolf zurück, indem er die unkonventionellen Nutzungsmöglichkeiten des sog. BodyBuggs zur Messung von Bewegung, Hauttemperatur, Hautspannung und Herzfrequenz bewarb, dass im Jahr 2008 ein absolutes Nischenprodukt aus dem Bereich der klinischen Medizin darstellte. ${ }^{66}$ Die Hackings der Quantified-Self-Community basieren seither darauf Technologien aus einem vordefinierten Anwendungsbereich in einen anderen zu übertragen. Auf diesem Weg sind während der letzten Jahre etliche experimentelle Selbstvermessungen entstanden, die (ob wissentlich oder nicht) in die tiefen Fußabdrücke der tayloristischen Betriebsforschung treten und vor allem Technologien aus den Bereichen Arbeit und Gesundheit immer weiter miteinander verschmolzen haben.

Wohingegen sich Kyrill Potatov durch die Integration pflanzlich-biologischer Indikatoren in die Zeit- und Produktivitätserfassung, eine Abbildung der psychischen Zufriedenheit mit den aktiven Office-Tasks verspricht, dient ihm ein anderes Technologie-Mash-Up aus Fitnessarmband und Terminplanern bei der Selbstkontrolle fernab des Schreibtisches. Auf seinem Twitter-Account hat er unter dem Titel »Turn your FitBit into a productivity coach « entsprechend eine Anleitung für das Hacking eines FitBit-Armbandes gepostet:

»Wouldn't it be great if your favourite productivity app could communicate with your Fitbit instead? Enter bitTicker: bitTicker makes your Fitbit vibrate whenever you get a notification from a chosen app. [...] You can use a scheduling app like TimeTune (for recurring tasks) or Any.do (for one-offs) to specify the exact day and time to do what you need to do. [...] You're in the kitchen making a sandwich and then your Fitbit vibrates. You look down at your wrist and read: >HISTORY ESSAY $\ll{ }^{67}$ 80412121302/-www.quantifiedself.com/ (zuletzt aufgerufen am 10.11.2017).

67 Quelle: https://twitter.com/kyrillpotapov, Post vom 28.08.2016. Hyperlinkziel des Posts: ww w.classroomhustle.com/-learning/sh-html/ (zuletzt aufgerufen am 10.11.2017[Hervorhebung im Original]). Die Hauptvorteile darin Benachrichtigungen dieser Art über somatische Impulse zu übertragen werden auf der Webpage wie folgt angegeben: »1. You can't do much with a Fitbit so it's not going to create more distraction. 2. It doesn't hold the negative associations of all the things you stress about on your phone. 3. It's right there on your wrist, holding you to account. «Wie so häufig, scheint der kommerzielle Erfolg des Unternehmens allerdings auch im Fall von Fitbit mit einer Schließung der durch es vermarkteten Technologien einherzugehen: »Fitbit have stopped allowing this feature on the Fitbit Charge. It only works on the Surge, Blaze and Alta« [Kursivsetzung im Original]. 
Die hierdurch in Aussicht gestellte technologisch externalisierte Verminderung von Wahlfreiheit artikuliert sich in diesem Bereich als Akt der Befreiung. Denn »im Zeitalter der unbegrenzten Möglichkeiten « in dem die Wahl zur Norm geworden ist, ist die Unsicherheit der Preis (Ehrenberg 2011: 249). Die Unabhängigkeits-, Selbsterkenntnis- und Emanzipationsrhetorik, unter deren Vorzeichen derartige Software meist verwendet wird, versinnbildlicht dabei aus einer Foucault'schen Perspektive sehr treffend das ambivalente Verhältnis zwischen verringerter Fremdbestimmung und einer hierdurch ins Werk gesetzten eigenverantwortlichen Steuerung, die als disziplinarische Kontrolle zweiten Grades durch die Kontrollierten selbst ausgeübt wird.

»And so you go to your desk and do the essay. Removing the choice of when you do the things you're meant to do can be very liberating! « ${ }^{68}$

Dieser sich auch über den Konnex zwischen Arbeit und Gesundheit hinaus vollziehende Brückenbau erscheint dabei diskursiv als technologische Verwirklichung eines breiter angelegten Vorhabens der produktiven Konfrontation einzelner, traditionell unabhängig geltender Lebensbereiche im Sinne eines Life Hacks. Ein Abbild dieser Entwicklung bietet etwa das Twitter-Profil Kyrills, in dem sich nur wenige Klicks von der Beschreibung des FitBit-Experiments entfernt Hashtags wie »\#lifehacks, \#bestversionofself, \#productivity, \#happy, \#success \#mondaymotivation, \#stayproductive, \#enhance, \#moreEfficient « ${ }^{69}$ usw. verästeln. Wenn die Arbeit Besitz von der Persönlichkeit genommen hat und alle Lebensbereiche nach ihren Prämissen organisiert werden, wenn also Arbeit zum Lebensinhalt wird, dann heißt produktiv zu arbeiten, produktiv zu leben: »\#productiveLife. ${ }^{70}$

D.h »Neue Arbeitsmethode und die Lebensweise lassen sich nicht von einander trennen: Es lassen sich keine Erfolge in einem der beiden Felder erreichen ohne spürbare Ergebnisse im anderen« (Brieler 2007: 257). Mit anderen Worten: »Die Arbeit wird mit Lust und Individualität aufgeladen und die Freizeit mit Handlungsmustern und Strategien aus der Welt der Arbeit gestaltet« (Diestelhorst 2016: 45). Die für den Diskurs der Kreativität mustergültige Figur der Künstlerin hat vorgemacht, wie sich im gemeinsamen Frame der Schaffensorientierung die vormals gegensätzlichen Organisationsprinzipen des materiellen Überlebens und solche der kulturellen und sozialen Selbstverwirklichung aufeinander zu bewegen (vgl. Abschnitt III. 2f.).

Auch der Post »Catherine Hooper on Hour Tracking for Priority Optimization « von Alexandra Carmichael auf der Quantified-Self-Webpage, lässt hinter der Be-

68 Quelle: https://twitter.com/kyrillpotapov, Post vom 28.08.2016. Hyperlinkziel des Posts: classroomhustle.com/-learning/sh-html/ (zuletzt aufgerufen am 10.11.2017).

69 Quelle: https://twitter.com/kyrillpotapov (zuletzt aufgerufen am 10.11.2017).

70 Quelle: https://twitter.com/kyrillpotapov (zuletzt aufgerufen am 10.11.2017). 
freiungsrhetorik die enge Verwobenheit von persönlichem Management und Persönlichkeit hindurchschimmern. Neben dem technischen Begriff der Optimierung greift sie den (für die projektförmige Arbeit ebenfalls konstitutiven) Begriff der Priorisierung auf und wendet beide gegen ihren intuitiven Gebrauch. So beschreibt sie wie sie ihren Alltag akribisch nach Tätigkeiten, Ereignissen oder Aufgaben scanned, die für sie von geringer Bedeutung sind um ihr Leben stärker nach ihren eigenen Prioritäten zu gestalten:

»Catherine Hooper has been tracking how she spends every hour of every day for the past 3 years. Why? To make sure she is living by her priorities. She defines her priorities, turns them into actions, then schedules them. [...] After canceling any things that don't fit, she adds in the actions that are important to her, in order of importance, as well as supporting actions that need to surround them. Her closing message? Don't have anything in your calendar that fails to meet your priorities! This is a great framework for saying no to unimportant things. $\ll^{71}$

Als ihre größte Priorität gilt dabei der Kategorie »Me«, in deren Dienst sie die Handlungen: »Exercise, Eating Right, Meditating, Personal Finances, Administration ${ }^{72}$ stellt. Das Beispiel zeigt daher einmal mehr auf, dass die »Macht der Abstraktion über uns« ihre Wirkung nicht verfehlt nur weil »wir sie anmaßend zu unseren eigenen erklären « (Klopotek 2016: 28).

Auch die in diesem Kontext bereits ausführlich diskutierte Plattform Daytum taucht im Diskurs dabei nicht ausschließlich unter Akzentuierung ihrer ästhetischen Merkmale auf, sondern als ein Produktionsort akribischer Fleißarbeit, dessen Disziplinareffekten sich die Self-Tracker*innen durchaus gewahr sind:

»The discipline it takes to collect that much data about yourself is quite the feat, but if you'd like to give it a try, you'll be interested in Nicholas' other project, Daytum, which is an application that allows you to do just that. ${ }^{73}$

So betrachtet, geht die Entfaltung der Persönlichkeit hier also zuerst den Weg der Selbsteinschränkung. Dieser offenkundige Widerspruch kann auf den ersten Blick sogar in Bezug auf das poststrukturalistische Vokabular der Machttechnologien irritieren, das sich mit der zunehmenden Liberalisierung demokratischer Staaten in Fragen der Organisation sozialer Prozesse von der disziplinarischen Kontrolle zu einer Lenkung individueller Freiheiten verschiebt. Obgleich die Disziplinarmacht (nicht nur im Zusammenhang des Self-Trackings sondern in weitaus mehr

71 Quelle: http://quantifiedself.com/2011/12/catherine-hooper-on-hour-tracking-for-priority-opt imization/ (zuletzt aufgerufen am 10.11.2017).

72 Quelle: https://vimeo.com/32106581 (zuletzt aufgerufen am 22.06.2018).

73 Quelle: http://flowingdata.com/2014/08/21/feltron-annual-report-2013/ (zuletzt aufgerufen am 10.11.2017). 
Bereichen des gesellschaftlichen Alltags) ein offenkundig und fast allgegenwärtiges Strukturmerkmal der Gegenwartsgesellschaft ausmacht, erscheint sie für ihre Beschreibung dennoch ungeeignet. Dies liegt sicher nicht zuletzt daran, dass der Term der Disziplin in den an Foucault anschließenden Arbeiten oftmals reduziert auf das Werk Überwachen und Strafen betrachtet wird und daher meist mit den totalen Institutionen des Gefängnisses und der ihnen teils funktional ebenbürtigen Anstalten, Schulen und Fabriken ${ }^{74}$ erscheint.

Disziplin wird so zu dem Ausdruck einer Machtasymmetrie zugunsten disziplinarischer Autoritäten verkürzt. Als Synonym für die Disziplinargesellschaft wird ihre organisatorische Bedeutung als Prinzip sozialer Kontrolle daher mit der zunehmenden Liberalisierung der Gesellschaft immer weiter auf einzelne Funktionen (wie z.B. das exekutive Gewaltmonopol und die Bekämpfung von Delinquenz) reduziert. ${ }^{75}$ Dabei werden die Grundlagen der disziplinarischen Kontrolle im Gegenteil mehr und mehr zu einem gesellschaftlichen Verhältnis und garantieren etwa die gouvernementale Kontrolle über die Gesundheit des Bevölkerungskörpers durch die Selbstdisziplin der Individuen. »Disciplinary techniques are »de-institutionalized « and dispersed throughout the social body« (Johnson 2014: 6). In Überwachen und Strafen schreibt Foucault daher auch von der sich selbst disziplinierenden Gesellschaft, deren alltägliche Praxen gegenseitiger Beobachtung und Überwachung sich quer durch die verschiedensten sozialen Bereiche verzweigen. »Foucault aggressively concludes: »We are ... in the panoptic machine« (Johnson 2014: 9). ${ }^{76}$ An dieser Stelle drängt sich ein Bezug zu der Sousveillance des Self-Tracking gerade zu auf, dass das Subjekt als spezifischer Fremd- und Selbstbeschreibungsmodus in Beziehung zu sich selbst setzt und es hierdurch gleichermaßen zur Wirkung und Voraussetzung seiner eigenen Subjektivierung macht, es dabei jedoch immer in bestimmte Ordnungen des Wissens einbindet (vgl. Bröckling 2012: 132). Dieses Paradox der affizierenden und affizierten Selbstwerdung hat Louis Althusser gleichnishaft durch das Bild der Anrufung zu verdeutlichen versucht, in dem

74 Wenn Foucault schreibt, dass »das Zellengefängnis mit seinem Zeitrhythmus [...] den Fabriken, den Schulen, den Kasernen, den Spitälern gleicht, die allesamt den Gefängnissen gleichen « (Foucault 1977: 292), so entsprechen diese gezogenen Parallelen durchaus auch den Vorstellungen des liberalen Gefängnisreformers Jeremy Benthams selbst. In »Panopticon; or Inspection House « schreibt er 1787: »[the] idea of a new principle of construction ... applicable to any sort of establishment: Prisons, Houses of industry, Workhouses, Poor Houses, Manufactories, Madhouses, Lazarettos, Hospitals, and Schools« (Bentham 1995: 29). Zitiert nach Johnson (2014: 8[Worteinfügung Th.M.]). Johnson zitiert hier aus der englischsprachige Ausgabe von Überwachen und Strafen (Foucault 1977b).

75 So kritisiert auch Neocleous: »Foucauldians use the police concept so abstractly that it comes to look as though it is yet one more synonym for »power «, »discipline«, and »governmentality« (2000: ix). Zitiert nach Johnson (2014: 22). 
ein Passant den sinnbildlichen Ruf eines Polizisten nur deshalb auf sich selbst zurechnet, da er sich selbst bereits als komplementäres Subjekt der Schuld und Unterwerfung imaginiert (Althusser 1977: 143) ${ }^{77}$ Althusser unterstreicht hier also die subjektheoretischen Aspekte von Kontrolle und Disziplinierung denen nach »die gesellschaftliche Bestimmtheit des Selbst als Selbstbestimmung zu begreifen « ist (Bröckling 2012: 134), also als die konstitutive Verbindung äußerer Zwänge mit der Selbstwahrnehmung und dem Selbstwerden.

So führen auch die Wahrheit und Objektivität versprechenden Zahlen der SelfTracker*innen für sich genommen noch nicht zu einer Veränderung von Gewohnheiten oder der Stabilisierung des gesundheitlichen und arbeitsorganisatorischen Höchstniveaus.

»A graph or a spreadsheet talks only in numbers, but there is a policeman inside all of our heads who is well equipped with punishing words. ${ }^{78}$

Das Self-Tracking der Quantified-Self-Community lässt sich damit auch als ein Beispiel dafür fassen, wie sich das nach Autonomie strebende Subjekt der Moderne in seiner Freiheit selbst begrenzt, indem es das geschichtlich keinesfalls obsolet gewordene Konzept der Disziplinarmacht innerlich aktualisiert. Und zwar in der nicht zwingend widersprüchlichen Verknüpfung von offensiver Auflehnung gegen die Wissensmonopole und die rigide Regelgeleitetheit biopolitischer Agenturen mit der intrinsischen Reproduktion ihrer Prämissen auf mikrosozialer Ebene im Maßstab N=1: $:^{79}$

\section{7 "A Calling to Account «"}

Die Thesen des ubiquitären Entrepreneurships haben sich inzwischen weit verbreitet, sodass sie interessanter Weise auch gerade im Kreis der Freelancer und eben der Self-Tracker*innen dazu verwendet werden kritisch untermalte Selbstreflexionen zu artikulieren, die (wie in Wolfs Polizeibezug) auch Formen des disziplinarischen Selbstzwangs umfassen. So wird z.B. die Infragestellung und Ablehnung gouvernementaler Autoritäten im Rahmen der Verdatung einzelner Lebensbereiche mitunter als etwas offengelegt, das im Rahmen des neoliberal geprägten Klimas der Individualisierung und der Selbstverantwortung in Form einer Erwartung an sie gerichtet wird. Vor allem durch die Sozialwissenschaftlerin

\footnotetext{
77 Zitiert nach Bröckling (2012: 134).

78 Quelle: www.nytimes.com/2010/05/02/magazine/02self-measurementt.html?_r=2\&ref=mag azine\&page-wanted=all (zuletzt aufgerufen am 02.09.2015).

79 Vgl.: http://quantifiedself.com/n1/ (zuletzt aufgerufen am 17.02.2015).

80 Vgl. Waring (1999[1988]).
} 
und das Quantified-Self-Mitglied Whitney Eric Boesel wird im Zusammenhang mit Self-Tracking zumindest schlaglichtartig die schwerhintergehbare Rekursivität einer auf Unangepasstheit basierenden Angepasstheit und einer kontrollfeindlichen Selbstkontrolle beleuchtet.

»Self-tracking, however, is not just an exercise in defying medical authority. The self-tracking subjectXresearcher also takes neoliberalism's preoccupation with The Individual and turns it inward, onto his own individual self « [sic!] ${ }^{81}$

Die diskurstheoretische Beschreibung einer allgemeinen numerischen Selbstprojektierung ist entsprechend kein Entlarvungsprogramm, das einer speziellen Subjektivität den Spiegel vorhält. Es zeigt vielmehr gerade auf, dass das Erkennen und Reflektieren von modernen Regierungsformen nicht automatisch von ihrem durchdringenden Einfluss befreit. Die Gründe hierfür lassen sich einerseits auf der Ebene von materiellen Strukturzwängen und marktwirtschaftlichen Konkurrenzmechanismen beschreiben, sie lassen sich andererseits aber auch diskursanalytisch aus der zirkulativen Verbindung individueller Selbstverständnisse und der Wirkung des Diskurses auf die Begriffe bestimmen, die sich Individuen von sich selbst machen. Denn weder der Diskurs, das Selbst noch die ihn begleitenden Praktiken sind einander vorgängig (Bröckling 2010: 24) sondern bedingen sich gegenseitig. In Bezug auf Praktiken der Selbstverantwortung und des Empowerment bedeutet dies, dass sie (selbst dann wenn ihr Ursprung in gouvernementalen Subjektivierungsprogrammen liegt) gerade auf der individuellen Inbesitznahme dieser Forderung basieren, wodurch sie mehr und mehr zu einem Selbstläufer werden. Die authentische Selbstermächtigung kann sich nur als etwas Innerliches und nicht als etwas äußerlich Auferlegtes plausibel artikulieren. Diskursiv erscheint das hierzu komplementäre Subjekt daher nicht selten als Resultat einer bewussten Entscheidung $^{82}$ (worin sich etwa die Losungen des Self-Esteem bereits erfüllen). Auch der durch die Programme der Mobilisierung des unternehmerischen Selbst unentwegt geforderte Nonkonformismus anstelle der stummen Regelbefolgung führt in

81 Quelle: „Empowerment Through Numbers? Biomedicalization 2.0 and the Quantified Self« http://thesocietypages.-org/cyborgology/2012/09/06/empowerment-through-numbersbiomedicalization-2-0-and-the-quantified-self/ (zuletzt aufgerufen am 10.11.2017).

82 Mit der teils in Zynismus umschlagenden Ironie der digitalen Bohemiens Holm Friebe und Sascha Lobo, wurde ein Versuch der Herstellung einer konsistenten Selbsterzählungen, die vor dem Problem steht, die Selbstbefreiung mit der gleichzeitigen Selbstunterwerfung zusammenbringen zu müssen, bereits angesprochen. IhrTraktat»Wir nennen es Arbeit« (2006) trägt eine Vielzahl unternehmerischer Anrufungen zusammen, um sie zu selektieren, zu sezieren, zu parodieren und die unternehmerische Anrufung schließlich als ein durchschautes Spiel im Modus des Als-Ob doch mitzuspielen. »Kritik perlt an ihnen ab, weil sie sie immer schon selbst formuliert haben« (Bröckling 2012: 140). 
ein ähnlich produktives Paradox, indem sie den Widerspruch selbst zum Innovationsprinzip erheben und der kritischen Abwendung schon dadurch zuvorkommen, dass sie ein anderssein fordern (Bröckling 2012: 141).

Es ist daher kaum verwunderlich, dass sich die mit der Quantified-SelfCommunity zusammenhängenden Selbstverständnisse im Diskurs überall dort, wo sie nicht ohnehin eine frappante Deckungsgleichheit mit den sie umsäumenden Leitbildern der unternehmerischen Unabhängigkeit aufweisen, als essentieller Kontrapunkt einer im Inneren liegenden, durch obsolete Wissensmonopole oder ökonomische Produktionstraditionen eingeschränkten Individualität konstituieren, die in Form völliger Kontrolle über alle sie betreffenden Belange gleichbedeutend mit einer immer schon im Innern liegenden Freiheit erscheint.

Dem flüchtigen Blick scheinen die Ambitionen der Quantified-Self-Bewegung möglicher Weise nicht weit entfernt zu sein von einer grundsätzlichen Kritik an humanwissenschaftlichen Macht-Wissens-Komplexen (vgl. Foucault 1981), die nicht zuletzt auch mit der Foucault'schen Prämisse in Einklang zu stehen scheint, dass »die Frage, ob man anders denken kann als man denkt, und auch anders wahrnehmen kann als man sieht, zum Weiterschauen und Weiterdenken unentbehrlich ist " (Foucault 1986: 15). Wo die postmoderne Theorie als Fluchtpunkt dieses Willens zum Wissen notwendiger Weise jedoch die Dekonstruktion des Subjekts entlang der epistemischen Integrationsstrukturen, Machtpraktiken und Wissensformen voraussetzt, ${ }^{83}$ scheinen die numerischen Vermessungstechnologien des quantifizierten Selbst, schon dem Namen seiner populärsten Community nach, vor allem ein Selbstverhältnis zu (re)produzieren das, wie auch Boesel bemerkt, im Zuge der Herausforderung biopolitischer Agenturen eine kritische Geneaologie ihrer numerischen Instrumente gerade ausspart und im Gegenteil die enthusiastische Aneignung ihrer Disziplinarpraktiken und Wissensformen bewirkt.

»The self-empowerment of self-quantifying, it seems, has less to do with the individual in the world and more to do with the individual in his own interiority; it

83 Siehe dazu Foucault: „Wenn das Wissenwollen heute seine größten Ausmaße annimmt, so nähert es sich nicht einer universellen Wahrheit; es verleiht dem Menschen keine sichere und ruhige Herrschaft über die Natur; im Gegenteil, es vervielfältigt die Cefahren, es zerstört die schützenden Illusionen; es vernichtet die Einheit des Subjekts; es befreit in ihm alles was auf seine Auflösung hinarbeitet« (Foucault 1978: 107f.). Und Bröckling: »Das Subjekt der Subjektivierung ist weder der letzte Zurechnungspunkt des Denkens, Wollens und Fühlens, noch imaginäres Personzentrum, in dem sich aller, Entfremdung zum Trotz ein authentisches Ich kristallisiert, noch gar potentieller Souverän, der sich nur erst von allen möglichen ,Kolonialisierungen< befreien muss« (Bröckling 2012: 132 [Hervorhebungen im Original]). 
may be less about challenging older relationships of authority and expertise, and more about coming to terms with the world as it stands. ${ }^{84}$

Entsprechend scheint der Einfluss aktueller Wissensregime, deren »Macht nicht zuletzt darin besteht, den Menschen eine Wahrheit über sich, über die Logik ihres Handelns und ihrer sozialen Beziehungen zu vermitteln « (Bröckling 2007: 14) in der Wissenskritik der Quantified-Self-Community ungebrochen zu sein. ${ }^{85}$

84 Quelle: „Empowerment Through Numbers? Biomedicalization 2.0 and the Quantified Self« http://thesocietypages.-org/cyborgology/2012/09/06/empowerment-through-numbersbiomedicalization-2-0-and-the-quantified-self/ (zuletzt aufgerufen am 01.02. 2018).

85 Auch Cruikshank resümiert entsprechend: »Self-esteem is a technology of citizenship and self-government for evaluating and acting upon our selves so that the police, the guards and the doctors do not have to« (1993: 330). 


\section{Quantrepreneure und der Individualismus der Prototypen}

\subsection{Quantified Self und die Verbreitung von Self-Tracking-Technologien}

Die Quantified-Self-Community ist nicht nur an der Entwicklung, sondern vor allem an der Verbreitung von Self-Tracking-Techniken und zugehöriger Technologien beteiligt. Als Inkubatorin bewirkt sie die Reifung von Ideen und beschleunigt im Sinne eines Accelerators die Entwicklung von Prototypen. Sie ist Anlaufpunkt für Nachwuchsprojekte, war besonders in ihren frühen Jahren die Ansprechpartnerin für die Massenmedien und ist bis heute auch selbstständige mediale Promotorin, ${ }^{1}$ Organisatorin weltweiter Treffen $^{2}$ und Lobbyorganisation für Kontakte in verschiedene Wirtschaftsbereiche und den Public Health Bereich. Die Mitglieder der Community sind Initiator*innen des Self-Tracking-Trends, Influencer und gleichzeitig auch Early Adopters der ersten kommerziellen Wearables und Software-Applikationen, die außerhalb oder über die Community hinaus vermarktet wurden.

Obgleich die verschiedenen Vermessungsmethoden, -technologien und -intentionen sehr divers sind, bzw. sie sich in Bezug auf technisches Entwicklungsniveau, Funktionsumfang und Kontinuität sehr unterscheiden, sorgt die Community für eine Popularisierung und Verbreitung des Selbstvermessungsthemas insgesamt.

Sie treten medienwirksam durch Interviews z.B. mit der New York Times in Erscheinung, sind auf Ted Talks zu Gast, stellen aktuelle Self-Tracking Projekte auf ihrem Blog zur Schau und sind personell verwoben mit dem Wired Magazine, dass die Reichweite der Community durch regelmäßige Artikel und Features von der ersten Stunde an kontinuierlich vergrößerte.

2 Während die meisten Meetups dezentral organisiert sind und keine explizite Absprache mit der Kernszene in San Francisco erforderlich ist, wurde dort dennoch ein Unternehmen gegründet, das z.B. die Organisation der europäischen oder globalen Konferenzen übernimmt: "QS Labs, LLC, is a California-based social enterprise that supports the Quantified Self movement worldwide.«Quelle: http://quantifiedself.com/aboutqs-labs/ (zuletzt aufgerufen am 07.06.2018). 
Vor allem das fluide Wechselspiel zwischen der unkonventionellen Entwicklungskultur der Quantified-Self-Szene und der Konsumgüterindustrie ist schon wenige Jahre nach der Community-Gründung in einen Kreislauf aus offener Diskussion von Vermessungstechniken, der individuellen oder proprietären Entwicklung dazugehöriger Soft- und Hardware, sowie der erneuten Erprobung (un)intendierter Nutzungsweisen und (Re)kombinationsmöglichkeiten mit anderen Vermessungstools, Anwendungsfeldern oder Datenquellen übergegangen, wovon nicht zuletzt auch angrenzende Marktsegmente profitieren. Und obgleich die Selbstdarstellung der Szene merklich auf einer scharfen Grenzziehung zwischen intrinsischer Selbstvermessungsbegeisterung und partiell partizipierenden Marktwirtschaftsinteressen basiert, war das gegenseitige Einflussnehmen von unabhängigen Entwickler*innen, kommerziellen Unternehmen und anderen Organisationen dennoch von Beginn an ein prägnantes Merkmal der CommunityEntwicklung. ${ }^{3}$

Dies führt immer wieder zu Spannungen, die sich zum einen auf der rein diskursiven Ebene in der Herausbildung von Selbstverständnissen und Semantiken, Rhetoriken und Begriffen äußern; zum anderen aber auch organisatorische und strukturelle Manifestationen erzeugen - wie z.B. die kontinuierliche Verstetigung von Unternehmensbeziehungen. Auch die im Jahr 2017 erstmals ausgerichtete SelfTracking-Messe während der globalen Jahreskonferenz in der San Francisco Bay Area ist hier zu nennen.

Diskursiv entstehen auch innerhalb der Community regelmäßig kritische Beurteilungen kommerzialisierender Entwicklungen, die sich meist zwischen den Extrempolen instrumenteller Marktinteressen und intrinsischer Experimentierfreude ansiedeln. Kritiken dieser Art richten sich nicht nur auf Versuche, Wearables, die ursprünglich im Netzwerk der intrinsischen Selbstvermesser*innen entwickelt und diskutiert wurden, gewinnbringend zu vermarkten, sondern legen allgemein eine Unterscheidung zwischen einer befreienden Selbstvermessung einerseits und einer Vermarktung von Selbstvermessungstechnologien andererseits $\mathrm{zu}$ Grunde. Letztere treten zudem häufig zusammen mit dem Verdacht auf, dass

3 Auszug aus der Beschreibung der Konferenz 2012 in Palo Alto: »We will also explore the potential effects of self-tracking on ourselves and society. If you are an advanced user, designer, tech inventor, entrepreneur, journalist, scientist, or health professional, please join us for a weekend of collaboration and inspiration!«Quelle: http://quantifiedself.com/conference/pal o-alto-2012 (zuletzt aufgerufen am 10.06.2013). In einer frühen Bestandsaufnahme aus dem Jahr 2010 zählte Gary Wolf an mehr als 200 Unternehmen, die über die verschiedenen Austauschformate der Community mit dem Diskurs verbunden sind: »l am working on a post that maps out some of the commercial territory I see being explored by the more than 200 companies that have been mentioned at the QS Show\&Tell meetings or here on our blog.«Quelle: http://quantifiedself.com/2010/03/quantified-self-business-model/ (zuletzt aufgerufen am 12.01.2016). 
Self-Tracking-Praktiken als neue Datenvorkommen erschlossen werden sollen und werden kongruent zu den kritischen Positionierungen gegenüber des Big-DataDiskurses mit Blick auf verschiedene Vermachtungsszenarien thematisiert (vgl Abschnitt III. 2.5.1):

»How can I get other people to collect data about themselves, and then use that data to get those people to do what I want them to do? ${ }^{4}$

Derartige Kritiken betreffen nicht nur Repräsentant*innen größerer Firmen, CoFounder oder »Venture Capitalists. « Vielmehr sind auch originäre Self-Tracker*innen, die ihre Innovationen einem Massenmarkt zugänglich machen, vor der Unterstellung einer kommerziellen Instrumentalisierung nicht gefeit, dernach sie das Tracking eigener Datenspuren lediglich als Multiplikatoren-Strategie verwenden um sich so innerhalb des Diskurses Gehör zu verschaffen. Insbesondere dann, wenn der oft schwer einzuhaltende Spagat zwischen einer durchaus erwünschten Enterprise Culture (in der sich persönlicher und beruflicher Erfolg vermischen) und Kommerzialisierungsambitionen misslingt, kann so schon das uneigennützige Zurverfügungstellen einer App als Strategie der unlauteren Datengewinnung über andere angesehen werden.

Sowohl die Beziehungen zu Technologieunternehmen als auch die verschiedenen Startups die aus der Community hervorgehen, wirken sich so auf die Konsistenz des Community-Selbstverständnisses aus - und das gleich in zweifacher Weise: Zum einen durch den irritierenden Effekt, den formale Beziehungen zu Unternehmen und politischen Institutionen auf das Unabhängigkeitsideal der Szene haben und zum anderen dadurch, dass die standardisierte Produktion von Trackern und Wearables dem DIY-Image entgegenläuft, das den Pioniergeist der Community diskursiv fest mit technischen Provisorien und Prototypen verbindet.

Das wichtigste und vorangigste Produkt der Community - die diskursive Verknüpfung von Self-Tracking-Technologien mit einem Narrativ der Authentizität und des Empowerment - wird durch die Kommerzialisierung bedroht. Dieser Konflikt bringt daher immer wieder sowohl diskursiv als auch institutionell ausgerichtete Strategien hervor, um kommerzielle Interessen ${ }^{6}$ von einem originären und authentischen Selbstverwirklichungs-Tracking zu trennen ohne dabei ein Inbeziehungtreten beider Bereiche zu verunmöglichen.

4 Quelle https://thesocietypages.org/cyborgology/2013/09/26/return-of-the-quantrepreneurs/ (zuletzt aufgerufen am 12.01.2016).

5 Quelle https://thesocietypages.org/cyborgology/2013/09/26/return-of-the-quantrepreneurs/ (zuletzt aufgerufen am 12.01.2016).

6 D.h. dem Verkauf der Daten einerseits und dem Verkauf der diese Daten generierenden Hardware andererseits. 
Das Projektplanungs- und Selbstsanktionierungs-Tool Beeminder stellt ein Beispiel für unternehmensseitige Strategien dar, dieser Diskrepanz zu begegnen. So wird über dieses Produkt versucht die disziplinarischen Motivationskonzepte der Quantified-Self-Community als Einnahmequelle zu erschließen. Beeminder ist kostenlos, solange die selbstgestecken Ziele eingehalten werden. ${ }^{7}$

Auf Seiten der Community basieren nicht-diskursive, d.h. primär organisatorische Versuche einerseits Gewinnstreben zu befördern ohne dabei den Sell-Out der Community voranzutreiben, meist auf turnusmäßig aufkommenden Abkopplungen kommerzieller Interessen aus einem intrinsischen Kern der Szene:

»It has been an amazing year since I stepped into the role of lead organizer for the Boston Quantified Self group. In that time we have seen the group over double in size, adding 700+ new members. Through the hard work of our dedicated team of organizers we have continued the tradition started by co-founder Cary Wolf and the Bay Area QS group of holding "show\&tells" where members can share their personal self-tracking stories, while also creating new event formats to serve the needs of our growing community. With events including our Health \& Wellness Innovation Night, The Quantified Patient, Women's Health event, and our Ideas to Action series we have been able to partner with amazing organizations including IDEO, athenahealth, Withings, Harvard i-lab, Bodymedia [...] and Endeavour Partners to bring the Boston QS community exciting events featuring world-class speakers and innovators. Due to this growth and expansion into additional areas of focus I have decided [...] to create the Self-Tracking group. The Self-Tracking group will be the connection point for industry professionals, researchers and users who are part of the emerging self-tracking ecosystem. This will allow the Boston Quantified Self group to remain focused solely on traditional show\&tells $[\ldots] . \ll^{8}$

Dabei wird schnell deutlich, dass sich die ideellen und ideengeschichtlichen Beziehungen zwischen Self-Tracking-Technologien und unternehmerischen Prämissen nicht in gleicher Weise trennen lassen. So dient auch die funktionale Aufteilung der Bostoner Quantified-Self-Szene letztlich einem Inbeziehungsetzen beider Gruppen über Sekundärwege. Sie ermöglicht einerseits ein direkteres Knüpfen von Geschäftskontakten und andererseits den Schutz des Produktionsortes unkonventioneller Technologieinnovationen vor dem Verdacht der kommerziellen Vereinnahmung und bewahrt den zugehörigen Unabhängigkeitsnachweis. Diskursiv kann so zwischen den idealtypischen Figuren der Self-Trackerin und der Ver-

8 Quelle: https://thesocietypages.org/cyborgology/2013/o9/26/return-of-the-quantrepreneurs/ (zuletzt aufgerufen am 12.01.2016). 
markterin eigener Technologien hin und her geswitcht werden, wobei sich auch die Zuhörerschaft etwaiger Vorträge (je nach Spezifikation des Treffens) entweder als Adressat technologieenthusiastisch gerahmter Selbstproblematisierungen oder kommerzieller Produktpräsentationen anbieten kann. Auf diesem Weg lässt sich so z.B. im Netzwerk der Boston Quantified Self Group eine individuelle Entstehungsgeschichte generieren, die sich in der Self-Tracking Group vermarkten lässt.

Auch diskursiv ausgerichtete Differenzierungen, die auf einer Unterscheidung zwischen unternehmerischen und intrinsischen Tracking-Projekten basieren, fallen letztlich hinter die Komplexität dieses Verhältnisses zurück. Dies sowohl dann, wenn sie mit entsprechender Kontrastschärfe als Form produktiver Aufgabenteilung beschrieben werden als auch dann, wenn im Sinne der politischen Facetten des Community-Verständnisses ein genuin »self-directed « Self-Tracking von den instrumentellen Ambitionen wirtschaftlicher Akteure unterschieden wird.

»As they discover hidden insights, it is the entrepreneurs who are bringing their findings - and their tools - to the masses. As self-trackers are pushing the movement forward, entrepreneurs are helping it scale. $\aleph^{9}$

Im Versuch, eine diskursive Unterscheidungsfähigkeit zwischen individuellen Selbstvermessungsambitionen und ihrer kommerziellen Überformung zu etablieren, verwendet Erin Boesel etwa die Wortschöpfung "Quantrepreneur. « ${ }^{10}$

Differenzierungsprobleme ergeben sich vor allem dadurch, dass sich die unternehmerische Grundierung der Community aber gerade nicht in formellen Geschäftstätigkeiten erschöpft. Die Diskussionen um eine Trennung zwischen kommerziellen und nichtkommerziellen Formen des Self-Tracking nimmt durch ihren starken juridisch-materialistischen Bias von den unternehmerischen Grundsatzprämissen der Quantified-Self-Community und ihrer vielschichtigen Verwobenheit mit dem rhetorisch-ideologischen Rahmenwerk des »Californien way of life« (Reckwitz 2010: 555) daher zu wenig Notiz. Die strikte Trennung zwischen intrinsischen (»self-directed«) Tracking-Projekten auf der einen und Geschäftsmodellen auf der anderen Seite bekommt den speziellen Modus nicht in den Blick, durch den hier elaborierte Problemlösungsfähigkeiten innerhalb eines zur Norm erhobenen Klimas der Kreativität und der Eigenständigkeit praktisch unter Beweis gestellt

9 Quelle: http://technori.com/2013/04/4281-the-beginners-guide-to-quantified-self-plus-a-listof-the-best-personal-da-ta-tools-out-there/ (zuletzt aufgerufen am 12.01.2016).

10 »I wish I could take credit for coining »quantrepreneur«, but I'm reasonably certain I picked it up somewhere along the line at either QSEU13 or QS2012; the term is a portmanteau of »quantified« and »entrepreneur«.«Quelle: https://thesocietypages.org/cyborgology/2013/09/ 26/return-of-the-quantrepreneurs/ (zuletzt aufgerufen am 12.01.2016[Hervorhebung im Original]). 
werden. Das quantifizierte Selbst, so wie es in den Tech-Blogs und CommunityEvents zu Tage tritt, ist als diskursive Kreuzung aus unternehmerischer Rationalität, kreativer Ambitioniertheit, Selbstständigkeitsstreben und historischen Traditionen der quantitativen Selbstwahrnehmung vielmehr zunehmend an einer ideellen Diskursfigur orientiert, die sich als QuantrepreneurIn treffend bezeichnen lässt. Der Begriff eignet sich über die eingeschränkte Verwendung innerhalb des Self-Tracking-Diskurses hinaus daher gut, um einen speziellen Modus des Selbstvermessens bzw. die mit ihm koinzidierende Subjektivität zu bezeichnen, die sich an den Kriterien der Projektförmigkeit sowie an verschiedenen Effizienz- und Optimierungsstrategien ausrichtet. Die Quantrepreneurin lässt sich entsprechend als eine Perspektivierung des quantifizierten Selbst lesen, die die unternehmerischen Implikationen stärker hervorhebt. Diskurstheoretisch gesprochen entspricht der Unterschied zwischen quantifiziertem Selbst und QuantrepreneurIn dabei in etwa der Unterscheidung zwischen unternehmerischem Selbst und UnternehmerIn: Wo das quantifizierte Selbst einen Modus der Selbst- und Fremdwahrnehmung beschreibt, der ohne numerische Werte inzwischen unvollständig wäre, allerdings niemals gleichbedeutend mit den an sie gerichteten Erwartungen ist, bezeichnet die Quantrepreneurin eine komplementäre Akteursfiktion: ${ }^{11}$ Eine spezifische Komposition aus Wünschen, Problemen und Fähigkeiten, die fiktiven Individuen durch Hardwarehersteller, Datenanalyst"innen, Personalentwickler*innen oder Manager*innen des Gesundheitssystems zugeschrieben werden. So wie das Ideal des Unternehmers subjektivierend auf das unternehmerische Selbst wirkt, wirkt auch die stereotype Quantrepreneurin, die sich aller sie betreffenden Probleme unternehmerisch und ergänzt um quantifizierende Problemlösungsmethoden zuwendet, subjektivierend auf das quantifizierte Selbst.

\subsection{A Community of like-minded others - Individuelles Tracking und überindividuelle Vergleiche}

Aus der gemeinsamen Verdatung anhand geteilter Apps und ihrer Standards ergibt sich für Quantified Self als »community of like-minded others" allerdings nicht erst ein diskursives Konsistenzproblem wenn Self-Tracking-Technologien mit pekuniärem Gewinnstreben verbunden werden, sondern schon dann, wenn es zur bloßen Verbreitung einer Self-Tracking-Technologie kommt - denn das individuelle Wissen, das Entwickler*innen über ihre Applikation besitzen führt innerhalb einer größeren Menge von Nutzer*innen zu einem informationellen Ungleichgewicht und bzw. schlimmsten Falls dazu, dass sich das Verhältnis zwischen Masse 
und zentralistischem Expert*innenwissen innerhalb des Self-Tracking-Diskurses reproduziert:

»Yet as a quantrepreneur, there's only so much marketing you can do before your (self-)marketing becomes telling someone else what to do, casting yourself as some kind of authority, or in some other way running afoul of someone else's individual autonomy. ${ }^{12}$

Aus einer Perspektive, die Individualismus mit Unabhängigkeit gleichsetzt, ${ }^{13}$ stellt sich im Grunde jede Befolgung von Ratschlägen oder die Anwendung nicht selbst erdachter Self-Tracking Verfahren als Beschneidung der eigenen Autonomie dar unabhängig davon, ob sich mit diesen Verfahren oder Technologien kommerzielle Strategien verbinden oder nicht.

Zudem stellt die Individualität der Self-Tracker*innen nicht nur ein zu erhaltendes Ideal, sondern in vielen Fällen auch ein strukturell auferlegtes Problem dar, das sich durch die Vereinzelungstendenzen moderner Arbeitsformen bedingt und das nun mit Self-Tracking-Technologien kompensiert werden soll. Die entsprechenden Hardware- und Software-Tools sind daher häufig so spezifisch, dass sie nicht ohne Übertragungsverluste zu einem Serienprodukt gemacht werden können.

»I remain convinced that attempts to translate the largely self-directed selftracking practices of Quantified Self into other-mandated self-tracking compliance tools will ultimately prove futile, because such attempts fail to take into account the personal, cultural, and structural reasons that self-directed selftrackers track in the first place. $\ll^{14}$

In Bezug auf die Produktion und Verbreitung standardisierter Self-TrackingTechnologien positioniert sich die Community daher ebenso ambivalent wie in Bezug zu überindividuellen Vergleichen individuell erhobener Daten.

Denn das Data-Sharing beschränkt sich, überall dort wo es als Austausch im Rahmen der Quantified-Self-Community ausgeflaggt wird, meist auf die ausschnittsweise Präsentation aggregierter und visuell aufbereiteter Daten, die z.B. als Slide-Show die sprachlich-numerischen Selbstdarstellungen während der Meetups begleiten.

12 Quelle: https://thesocietypages.org/cyborgology/2013/09/26/return-of-the-quantrepreneurs/ (zuletzt aufgerufen am 12.01.2016).

13 »The implied or imagined »self « of Quantified Self is almost always an individual self, an independent and autonomous self.«Quelle: https://thesocietypages.org/cyborgology/2013/09/ 26/return-of-the-quantrepreneurs/ (zuletzt aufgerufen am 12.01.2016 [Hervorhebung im Original]).

14 Quelle: https://thesocietypages.org/cyborgology/2013/09/26/return-of-the-quantrepreneurs/ (zuletzt aufgerufen am 12.01.2016). 
Daten dieser Art bieten hier das Potential von einer indirekten Ressource $\mathrm{zu}$ einer Ressource zu werden, die direkt in Wert gesetzt oder zur Erhöhung von sozialem Prestige eingesetzt werden kann. Die subjekttheoretisch apostrophierte Quantrepreneurin kann sich als kalkulatorisch versierte Unternehmerin ihrer Selbst nicht nur auf ihre eigene Vermessungshistorie berufen, um qua Training und organisatorischen Eingriffen die eigene Leistungsfähigkeit zu verbessern; sie ist durch die Daten auch in der Lage ihre Leistungsfähigkeit unter Beweis zu stellen und sich in bestimmten Kontexten (unter Adaption der dort geltenden Prämissen) z.B. als funktionale und berechenbare Arbeitskraft, als gesunder und körperbewusster Mensch oder als selbstvergessener Workaholic zu konstituieren. Extrembeispiele stellen z.B. Zeitraffer-Videos aus Bildschirmmittschnitten dar, die von den Self-Tracker"innen (mit der gewohnten Absicherung durch eine ironische Doppelbödigkeit) als »Productivity Porn $\aleph^{15}$ bezeichnet werden. Unter Titeln wie »We're Up All Night To Get Stuff Done ${ }^{16}{ }^{16}{ }$ Maniac Weekend ${ }^{17}$ oder $»$ Maniac Week ${ }^{18}$ stellen sie Zeitraffer-Videos ihres letzten Arbeitsmarathons ins Netz - wobei auf den Screenshots der Arbeitsvorgänge immer wieder die Dashboards von RescueTime oder Beeminder aufflackern.

Wo die analytische Selbstverdatung am ehesten dem Human-RessourceManagement des eigenen Unternehmens entspricht, stell diese Form der Zweitverwertung das Marketing dar, das potentielle Geschäftskontakte über die Qualitäten des Unternehmens informiert. Der kompromisslose Individualismus der Quantified-Self-Community stößt hier allerdings auf ein Problem, denn der hierdurch entstehende Wert stellt eine relationale Größe dar; entsprechend stellt sich auch der Nutzen vieler Leistungs-Scores erst im Vergleich mit anderen ein. Der Bedarf an vorstandardisierten Selbstvermessungstechnologien, die bestimmte Formen der Berechnung von Vergleichswerten und ihre öffentliche Distribution ermöglichen, kann daher erst im Zuge ihrer Verbreitung über den Kreis der Entwickler*innen und aktiven Promoter*innen hinaus bedient werden. Umgekehrt lässt sich sagen, dass sich die (Re)integration von überindividuellen Vergleichswerten in den Self-Tracking-Diskurs parallel zu einer steigenden Popularität des Themas und der Verbreitung standardisierter Self-Tracking-Technologien vollzieht.

15 Quelle https://blog.beeminder.com/maniac. An anderen Stellen auch als»Entrepornography« bezeichnet. (Zuletzt aufgerufen am 12.01.2016).

16 Quelle: https://youtu.be/IcYQUXozxOA Der Titel bezieht sich auf die Textzeile »We're up all night to get lucky« aus dem Titel »Cet Lucky« von Daft Punk (zuletzt aufgerufen am 12.01.2016).

17 Quelle: https://youtu.be/giBIJW-2hro (zuletzt aufgerufen am 12.01.2016).

18 Quelle: https://youtu.be/ODhx-CbXglg (zuletzt aufgerufen am 12.01.2016). 
Wohingegen die Messungen der Quantified-Self-Mitglieder vornehmlich auf einer kontemplativen und im engeren Sinne selbstbezüglichen Orientierung an den Datenspuren vergangener (eigener) Tätigkeiten, Gefühle oder Körperwerte beruhen, die sie anhand selbst definierter Ziele und Grenzwerte bewerten, treten im Zuge der zunehmenden Vernetzung und Akkumulation dezentraler Selbstvermessungen nun immer mehr Bewertungskriterien hinzu, die sich vor allem aus überindividuellen Durchschnitten ableiten lassen und es den Vermessen(d)en ermöglichen selbst aktiv kleinteilige Details über verschiedene Aspekte ihres Lebens in ein Verhältnis zu anderen zu setzen. Viele der inzwischen massenhaft genutzten und kommerziell betriebenen Selbstvermessungsportale basieren sogar schwerpunktmäßig darauf, Protokolle über Bewegung, Gefühlszustände oder Körperwerte der Nutzer*innen in standardisierten Kategorien wie Kilometern, Kalorien oder sog. Mood-Scales auf einem entsprechenden Webportal zu speichern und mit den Werten anderer Nutzer*innen zu vergleichen. Während innerhalb der QuantifiedSelf-Community neue Vermessungspraktiken noch in institutionalisierten Treffen wie z.B. den Show\&Tell-Formaten durch konsistente Beschreibung von Vermessungsweisen und zugrundeliegender Bedarfe fundiert wurden, ${ }^{19}$ werden individuell festgelegte Ziele, im Zuge der massenhaften Vernetzung von Vermessungstechnologien, nun sukzessive durch die integrierten Mittelwerte auf den Displays der Smartphones ergänzt. Die Möglichkeit sich selbstbezüglich mit den Daten auseinanderzusetzen, eigene Fortschritte und Rückschritte zu beobachten oder sich Ziele zu setzen, korrespondiert so immer stärker mit der fortlaufenden Beobachtbarkeit der Fortschritte und Rückschritte aller übrigen Nutzer*innen im (zumindest plattformweiten) Durchschnitt. Die großen Datenaggregate vernetzter VermessungsApps bieten über die Möglichkeiten hinaus gezielt auf sich einzuwirken, daher häufig selbst die nötigen Kennwerte, an denen sich der erforderliche Grad an Selbstjustierungen, z.B. bei der Änderung von Ernährungsgewohnheiten, Arbeitsweisen oder Körperroutinen in Relation zu den Werten Anderer bemessen lässt.

Seit 2007 sind viele Technologien entstanden, die automatisiert und cloudbasiert zahlenbasierte Vergleichszusammenhänge anbieten. Viele von ihnen lassen sich als das indirekte Ergebnis des jahrelangen diskursiven Austausches rund um Quantified Self betrachten oder sind aus den individuellen Selbstverdatungsprojekten einzelner Mitglieder hervorgegangen.

Ein weithin symptomatisches Beispiel stellt das bereits erwähnte RescueTime dar, das über den Co-Founder Robby McDonell eine enge Verbindung zur Quantified-Self-Community aufweist, dort im Rahmen verschiedener Panels, Breakout-Discussions oder Talks bis heute immer wieder eine Rolle spielt, aber

19 Zu Beginn jedes Show\&Tell-Plenums der Quantified Self Conference 2014 in Amsterdam, wiederholte Mitorganisator Gary Wolf die drei Kernfragen, an denen sich die Vorträge orientieren sollen: "What did you do? How did you do it? What did you learn?« 
eben auch eine große und weltweit verstreute Menge an Nutzer*innen hinter sich versammelt, die nicht in den semiprofessionellen Strukturen der Quantified-SelfCommunity vernetzt sind oder von diesen möglicher Weise noch nie etwas gehört haben.

»We started telling people about the little nerdy analytics toy that we had built, and to our surprise a lot of people said it sounded really cool and they'd like to try it themselves. That was the point when we realized it might be an interesting product to build for others. ${ }^{20}$

Spätestens an diesem Punkt der diskursanalytischen Spurensuche erweitert sich die Perspektive auf das Self-Tracking damit auch von der vergleichsweise engen Fokussierung auf eine diskursiv sehr präsente aber zahlenmäßig kleine Avantgarde hin zur Betrachtung des Mainstreaming der Self-Tracking-Technologien, also der viel allgemeineren Bedeutung die diesem Phänomen inzwischen in anderen gesellschaftlichen Bereichen zukommt. ${ }^{21}$

\subsection{Selbstwertsteigerung und Ressourcenplanung im Ich-Unternehmen}

So bietet RescueTime über das bereits angesprochene Repertoire an Funktionen für die selbstreferentielle Optimierung des individuellen Arbeitstages hinaus auch die Akkumulation überindividueller Messdaten an. Vergleichsmaße wie Mittelwerte setzen Nutzer*innen untereinander in Beziehung und machen die durch sie

20 Aus einem Interview mit Robby McDonell. Quelle: http://lifehacker.com/im-robby-macdonel I-and-this-is-the-story-behind-rescu-1497037183 (zuletzt aufgerufen am 14.11.2017).

21 Dabei ist die Tatsache, dass sich die durchschnittliche Nutzerin innerhalb dieses Diskurses sprachlich kaum zu Wort meldet, sondern allenfalls als anonymisierter Zahlenwert in den Nutzungsstatistiken auftaucht oder in ähnlich normalisierter Weise durch die pauschalen Zitationen des Marketings massentauglicher Produkte präsent ist, gleichermaßen Ergebnis als auch Defizit der Diskursanalyse: »RescueTime teaches me things about my workday I would never otherwise have the time or perspective to learn. It's a great tool for anyone who wants to be intentional about improving their productivity. «Quelle: https://www.rescuetime.com/ (zuletzt aufgerufen am 26.06.2018). Obgleich die Methode (anders als z.B. ethnografische Interviews) nicht in der Lage ist Stellungnahmen, Positionierungen oder Bewertungen aktiv anzuleiten und sich auf das bereits Dokumentierte beschränken muss, spiegelt sie gleichzeitig wieder, wie und in welchen Modi derartige Technologien auch für andere passive Rezipient ${ }^{*}$ innen im Diskurs erscheinen. So bleibt der Modus in dem Technologien wie RescueTime (fernab des Marketings) diskursiv erscheinen, deutlich durch einen avantgardistischen Bias bestimmt, der die Darstellung, Kommentierung und Bewertung dieser Technologien meist mit der Beschreibung individueller Aneignungspraktiken oder experimentellen (Um)Nutzungen verbindet. 
aufgewendeten Arbeitsstunden, die Effektivität oder Kontinuität ihrer Arbeitszeit vergleichbar.

Über selbstbezügliche Formen der Kontrolle hinaus (die einzelne Nutzer*innen anhand von Balkendiagrammen und Tabellen über Höhen und Tiefen der individuellen Leistungsfähigkeit während der vergangenen Tage, Wochen oder Monate informieren), bietet das Portal auch die Homogenisierung einzelner Praktiken, Zeitabschnitte, Tages- und Wochenzyklen zu einem Normalfeld an, innerhalb dessen über Skalierungen, vor allem aber über Prozentwerte, Vergleichbarkeit mit anderen Nutzer*innen ermöglicht wird - so z.B. durch den sog. »Produktivitätsmittelwert. ${ }^{22}$ Inhaber*innen von RescueTime-Accounts können sich bis auf einzelne Perzentile genau in der Produktivitätsnormalverteilung der Gesamtnutzer*innen, spezifischer Berufsgruppen oder Belegschaften verorten. Durch die standardisierte und thematisch begrenzte Erfassung von einzelnen Tätigkeiten und den Zeitspannen, die für sie aufgewendet werden, operationalisiert das Portal den Anschluss an den (meist) anonymen Cluster der Plattform-Nutzer*innen, wobei die persönlichen Ziele in unmittelbarer Nähe zur Leistung des "Avarage-Users« stehen.

In den entgrenzten Arbeitsverhältnissen jenseits von traditionellen Nine-toFive-Jobs, die häufig mit einem hohen Maß an Isolation und Vergleichsschwierigkeiten einhergehen, dient ein Tool wie dieses nicht nur in der bereits beschriebenen Weise dazu, den Arbeitsalltag zu (re)strukturieren, sondern bietet vielmehr durch das vergleichende Inbeziehungsetzen einzelner Selbstverdatungen Orientierungshilfen, die z.B. die annäherungsweise Einschätzung darüber ermöglichen, wieviel wöchentliche Arbeitszeit für eine bestimmte Branche als normal gilt. Die Rationalisierung von Tätigkeiten und ihre Übersetzung in konkrete Werte, denen der Anschein des Faktischen innewohnt, stellen dabei den Anschluss an die zahlenförmige Sprache der modernen Arbeitswelt sicher, ermöglichen eine Selbstherstellung, die von der eigenen subjektiven Einschätzung unabhängig erscheint und liefern die Grundlage für die Bestimmung des eigenen Marktwertes und eben auch des Marktwertes anderer. ${ }^{23}$

Damit ist der durch Patricia Clough formulierten Kritik an Hardt und Negris (2002) Behauptung darüber zuzustimmen, dass immaterielle Arbeit grundsätzlich

22 Wie Link ausführt, kommt es zu einer Homogenisierung durch die Verdatung von Populationen unter selektiven Prämissen (Link 2001: 82).

23 Auch wenn für die konkrete Überführung geleisteter Arbeitsstunden in Geldwerte meist andere Programme bevorzugt werden: »Therefore, RescueTime isn't going to replace Time Doctor as my preferred service for tracking my work hours (mainly for tax purposes and financial records). Although it does an excellent job helping analyze productivity and helping you determine how to work smarter in the future.«Quelle: https://thetechreviewer.com/serv ice/rescuetime-pro-review-time-management-productivity-tracking/ (zuletzt aufgerufen am 10.11.2017). 
nicht messbar sei (vgl. Clough 2013: 113). Unabhängig von Objektivitäts- und Validitätsfragen zeigt sich, dass die so kreierten Werte eine strukturierende Wirkung auf den Diskurs und die nicht-diskursiven Praxen der Wissensarbeit haben.

Spätestens an dieser Stelle erfüllt sich dann auch für den Großteil der technisch weniger versierten Nutzer*innen die zentrale Losung jedes Empowermentprogramms als Selbstwert. Dies allerdings im doppelten Sinne: Nämlich einerseits als ein Gewahr werden über die eigenen Fähigkeiten und andererseits als Nachweis über diese Fähigkeiten, der sich auf dem Markt in Geldwerte transformieren lässt. Eine solche Besser-Schlechter-Bestimmung koppelt die Selbstaufwertung allerdings an die Abwertung der Vergleichsprofile und umgekehrt. Im Falle eines so spezifischen und selektiv ausgerichteten Erhebungs- und Vergleichsinstruments wie RescueTime ist eine solche dynamische Verteilung gleichbedeutend mit flexiblen Konkurrenzindikatoren, die nicht zuletzt das Potential bergen zu einem zirkulären Wechselverhältnis gleichzeitiger Erzeugung und Erfüllung von Verbesserungsansprüchen beizutragen.

Insbesondere mit Blick auf die Mittelwertberechnungen solcher Portale wird dabei deutlich, dass in der Peripherie der Quantified-Self-Community klassische bevölkerungspolitische Instrumente wieder mit den Methoden der individuellen Verdatung verwoben werden. Obgleich hier zunächst keine organisationelle Verbindung oder ein direkter Rekurs auf das durch biopolitische Agenturen bereitgestellte Wissen erfolgt, überträgt sich doch ihr allgemeingültiger Mechanismus (die eigendynamische Wirkmächtigkeit numerischer Vergleiche ${ }^{24}$ in den Bereich des Self-Tracking - wodurch sich die Bedeutung des Selbst verschiebt. Ein Gutteil der subjektivierenden Eigenschaften dieses Programms liegt damit in Funktionen, die überhaupt erst mit der massenhaften Verwendung einer standardisierten Software möglich werden.

Obgleich die Zahlen-Cluster von RescueTime nur einen sehr selektiven und homogenisierten Bereich abbilden, ermöglichen sie die vergleichende Einordnung in einen Gesamtzusammenhang und somit die Erzeugung eines funktionalen Selbstverständnisses. Ein Selbstverständnis das kongruent zu den Funktionen der Gesundheits- oder Arbeitsmarktstatistiken graphisch aufbereitete Wissensordnungen rezipiert, welche dazu anleiten, sich auf eine bestimmte Weise $\mathrm{zu}$ verstehen und bestimmte Routinen mehr zu pflegen als andere. Allerdings lassen diese interaktiven Systeme die statistische Repräsentation der eigenen Position innerhalb einer Verteilung dynamisch erscheinen. Anders als in Relation zu den enorm abstrakten Ausschnitten großer Sozial-Panels, sowie sie in den Massenmedien dargestellt werden, sind die Konsument*innen der Durchschnitte unmittelbar auch ihre Produzent"innen. Strategien des Einwirkens auf sich selbst können hier unmittelbar Feedback-gestützt getestet und verändert werden. Umgekehrt wird 
das Prinzip des zahlenförmig operierenden Normalisierungszusammenhangs stärker an Technologien des Selbst gekoppelt, wobei die Hervorbringung von statistischer Normalität und ihre Beobachtung nun die gleichen technischen Kanäle nutzen.

\subsection{Work/Workout-Balance - Resilienzstrategien im Home Office}

Im fluiden Zyklus der Projektarbeit, in dem Statuspositionen und Funktionsrollen ständig neu verteilt werden und die Arbeitskraftunternehmerin zusätzlich zur Unternehmerin der Arbeitskraft anderer werden kann, wechselt auch das Tracking mit ähnlicher Häufigkeit die Register. Die Anwendung von Selbstüberwachungstechnologien zur Überwachung ${ }^{25}$ anderer ist im Bereich der Nutzung vorstandardisierter Massenprodukte wie RescueTime oder Time Doctor daher eine häufig anzutreffende Verschiebung.

»Keeping your employees productive while working online is a difficult task for many business owners. [...] By signing up with Time Doctor, you will be able to automatically track your employee's hours of work and productivity levels in a straightforward and simple way. By accessing the Time Doctor's control panel via the web, you can view on-demand reporting, analysis, and productivity levels make sure your employee's are not wasting your valuable time and money. ${ }^{26}$

Das Tracking der Produktivität im Rahmen hierarchischer Arbeitsbeziehungen basiert hier einerseits auf der konzeptionellen Ähnlichkeit, die derartige TrackingTools zu den schon lange eingesetzten Zeiterfassungssystemen größerer Unternehmen aufweisen, allerdings auch darauf, dass sich diese Technologien in einer Kategorie mit Selbstvermessungstechnologien verorten lassen, wodurch sich die Diskrepanz zwischen einem affirmativ aufgeladenen, auf Freiheit basierenden Arbeitsverständnis und vergleichsweise rigiden Kontrolltechnologien diskursiv auflösen lässt.

Gerade im Bereich von Startups oder projektbezogenen Arbeitszusammenhängen, in denen auch Positionen wie Employer und Employee bzw. Festanstellung und Selbstständigkeit häufig in dichter Folge wechseln, entwickelt sich aus der Selbstvermessung zudem auch eine Legitimitätsformel für die Vermessung anderer, die darauf basiert, dass man Methoden der datenbasierten Leistungsüberwachung jederzeit auch auf sich selbst anwenden würde - und dies möglicher Weise

25 Oder der Überwachung der Selbstüberwachung anderer.

26 Quelle: https://thetechreviewer.com/service/time-doctor-review-online-time-tracking-analysis/ (zuletzt aufgerufen am 15.03.2018[Hervorhebung im Original]). 
auch tut. Wie Foucault in Bezug auf die gouvernementale Steuerung von Selbststeuerungen schreibt, sind die Fragen "wie sich regieren, wie regiert werden, wie die anderen regieren [...]? « seit jeher fest verbunden (Foucault 2004[1977-1978]:135).

Die Tatsache, dass das Thema der Überwachung von Angestellten in dieser Weise überhaupt angesprochen wird, verdeutlicht für sich genommen bereits, dass es sich um eine erklärungsbedürftige Praktik handelt, die nicht von vornherein Legitimität für sich beanspruchen kann. Es handelt sich um einen Auslotungsprozess dessen, was gegenwärtig als akzeptabel gilt und um eine Suche jener argumentativen Strategie, die sich am besten eignet die Überwachungsmaßnahmen zu rechtfertigen. Zwischen den Zeilen gelesen bedeutet dies, dass die Überwachung der Selbstüberwachungen erfordert, dass man sich selbst sichtbar den gleichen Normen und Sachzwängen unterstellt, die man zur Kontrolle anderer heranzieht. Was für den Nachgang der gebürtigen Souveränität galt, scheint somit auch noch für den Nachgang der hierarchischen Angestelltengesellschaft zu gelten: Wer andere regieren will, muss sich selbst regieren bzw. wer andere überwacht, legitimiert dies dadurch auch sich selbst überwachen zu lassen:

»I know this might be a privacy issue to some, but personally l'd want every dollar in compensation to be rightfully earned. Personally, it has aided me in becoming more productive and staying focused on the task at hand. I highly recommend the Time Doctor service to anyone looking to actively track their own time and productivity or anyone who hires a freelancer, consultant, or telecommuter on a regular basis. « ${ }^{27}$

Im Scharnier zwischen eigenverantwortlicher Selbstständigkeit und der Arbeit in den temporären Teams von Kleinstunternehmen bereitet sich über Beispiele der eben beschriebenen Art ein diskursiver Zirkelschluss vor, der das posttraditionale Arbeitssubjekt der Selbstverwirklichung wieder mit den Kontrolltechniken der Angestelltengesellschaft verbindet. Und das meint in erster Linie das hervorgehobene Interesse, das biopolitische Agenturen an den verschiedenen Beziehungen zwischen Produktivität und der Gesundheit der Arbeitenden haben. Während die Analyse der Leistungsbedingungen den Verkauf der eigenen Arbeitskraft kongruent zum Kauf fremder Arbeitskraft erleichtert, wird die Pflege der Arbeitsfähigkeit (ganz im Einvernehmen mit dem Optimierungsverständnis der Betriebswissenschaft und der Humankapitaltheorie) dabei zu einer werterhaltenden Maßnahme.

27 Quelle: https://thetechreviewer.com/service/time-doctor-review-online-time-tracking-analysis/ (zuletzt aufgerufen am 15.03.2018). 
»The secret to being as productive as possible is striking the right balance between taking care of ourselves while maximizing the time we spend working at peak productivity « $[$ sic! $] .^{28}$

In dieser marktwirtschaftlichen Peripherie scheint das im Diskurs sehr populäre »Wir« seine Bedeutung verschoben zu haben und weniger an dem vergleichsweise elitären Kreis der Quantified-Self-Mitglieder und dafür mehr an einem »Wir« $\mathrm{zu}$ liegen, von dem aus im Namen aller Menschen gesprochen wird, die mit ähnlichen Problemen konfrontiert sind und denen folglich ähnliche Bedürfnisse attestiert werden. Dort wo der Sprechort näher an Technologieunternehmen, Marketingfirmen oder staatlichen Instituten liegt, transformiert sich das exkludierende Community- »Wir« zu jener diffusen Inklusionskategorie, die durch die $>\mathrm{N}=1$ Experimente als Allgemeinheit politisiert und kritisiert wird. Hier wird das Subjekt vielmehr wieder vor allgemeine Tatsachen gestellt, die sich (klassisch gouvernemental) im Modus des Drängens innerhalb des Diskurses verbreiten (vgl. Foucault 1981[1969]: 39). So z.B. über den Rekurs auf eine Studie des Centers for Disease Control and Prevention:

»In a pair of recent studies, the CDC called attention to two very alarming trends. Americans are not getting nearly enough exercise and a majority fall many hours short of the recommended amount of sleep. Not only is this bad for our health, it harms our productivity as well. $\ll^{29}$

Selbstquantifizierung erscheint hier zwar immer noch als Lösung der behandelten Probleme, jedoch nicht um die Durchschnitte und Verallgemeinerungen der gouvernementalen Agenturen im gleichen Maß herauszufordern, sondern um ihnen im Sinne einer bevölkerungspolitischen Kongruenzbeziehung nachzueifern.

Bevölkerungspolitische Prämissen werden hier nicht mehr auf Individualebene reproduziert, sondern durch den Normalismus Link'scher Kurvenlandschaften (vgl. Link 2001: 88) breiter statistischer Orientierungsmaßstäbe gerahmt - wobei die überall verfügbaren und gesellschaftsweit popularisierten Self-TrackingTechnologien als adäquates Mittel angepriesen werden, für sich selbst jene Mängel zu beheben von denen der Durchschnitt der Gesellschaft als Ganzes betroffen ist.

Die richtige Balance aus Arbeit und Reproduktion erfordert dieser Logik nach daher auch das richtige Mashup aus Produktivitäts- und Fitness-Apps, die

Quelle: http://blog.sumall.com/journal/science-productivity-using-fitness-apps-like-fitbit-can -impact-perfor-mance-work.html (zuletzt aufgerufen am 15.03.2018).

Quelle: http://blog.sumall.com/journal/science-productivity-using-fitness-apps-like-fitbit-can -impact-performance-work.html Direktlink zu der erwähnten Studie des Centers for Disease Control and Prevention: »CDC 24/7: Saving Lives. Protecting People«: https://www.cdc.

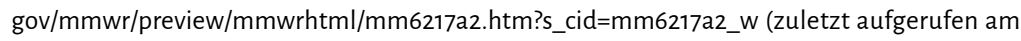
15.03.2018). 
neben dem numerischen Beobachtungs- und Kommunikationsmodus sowie der analytischen Detailtiefe auch die motivationalen bis disziplinarischen Aspekte der Selbsterhaltung aus dem Kreis avantgardistischer Self-Tracker*innen importiert und über den Markt verbreitet.

»Seeing that blank space on your fitness app's progress chart or the blinking light on your Fitbit can serve as subtle yet powerful motivator. Not only will reminders like this help you keep consistent with your active lifestyle, they will also help cement the corresponding gains in your overall productivity. ${ }^{30}$

Auch das Unternehmen Fitbit, dem heute nachgesagt wird, als eines der ersten kommerziell erfolgreichen Startups aus der frühen Phase von Quantified Self hervorgegangen zu sein, ist als globaler Inkubator und öffentlichkeitswirksamer Promotor bestimmter Definitionsweisen von Selbstvermessung bzw. damit verbundener Selbstverständnisse heute sehr präsent im Diskurs vertreten und trägt durch eine standardisierte Produktpalette $\mathrm{zu}$ einer erleichterten praktischen Adaption des Self-Tracking in unterschiedlichen Gesellschaftsbereichen bei; Ideen und Legitimität bezieht das Unternehmen dabei allerdings zu einem Gutteil aus dem dezentralen Basissegment der Self-Tracking-Szene, dessen Popularisierung es gleichermaßen vorantreibt.

Dabei ist festzustellen, dass im Bereich der Kommerzialisierung von SelfTracking-Technologien auch ehemals szeneinterne Bottom-Up-Projekte wie Fitbit, die als Teil der Quantified-Self-Bewegung mit der Herausforderung von Wissensautoritäten verbunden waren, nun auf wissenschaftliche und teils normative gesellschaftliche Konventionen wie den Quetelet-Kaup-Index (besser bekannt als Body-Mass-Index) rekurrieren oder durch Voreinstellungen in der Gerätesoftware Grenzmarker und Soll-Werte definieren - so z.B. das meist willkürlich erscheinende Mindestmaß an Schritten, dass laut den Lauf-Apps pro Tag absolviert werden soll. ${ }^{31}$

Hersteller wie Fitbit oder Withings gehen zudem inzwischen Kooperationen mit großen global agierenden Firmen ein, um Self-Tracking-Technologien über maßgeschneiderte Hardware-Software-Angebote oder Gesundheitsprogramme unter den Belegschaften zu verbreiten.

»Gesundheit der Mitarbeiter verbessern. Mitarbeiterproduktivität steigern. $\ll^{32}$

30 Quelle: https://thetechreviewer.com/service/time-doctor-review-online-time-tracking-analysis/ (zuletzt aufgerufen am 15.03.2018).

31 »Die Partnerschaft kombiniert den wissenschaftlich fundierten Ernährungsansatz von Weight Watchers mit den marktführenden Activity Trackern von Fitbit, um Lösungen für ein gesünderes und aktiveres Leben anzubieten.«Quelle: https://www.presseportal.de/pm/4354 5/2764371 (zuletzt aufgerufen am 19.11.2015).

32 Quelle: www.fitbit.com/de/fitbit-wellness (zuletzt aufgerufen am 19.11.2015). 
Die Service- und Beratungssparten Withings Corporate Wellness $360^{\circ}$ oder Fitbit Wellness $^{33}$ sind nur zwei konkrete Beispiele dafür, wie sich der breite Diskurs über die numerische Erfassung von Produktivität und Fitness über Begriffe wie Wellness inzwischen mit angrenzenden Diskursen, wie etwa der Personalentwicklung oder des betrieblichen Gesundheitsmanagements verzweigt. Die Selbstbeschreibungen dieser Firmen und Programme basieren allerdings gerade darauf, die Verweise auf den individualistischen Selbstvermessungsenthusiasmus der Quantified-SelfSzene in den Narrativen ihrer Firmenentstehung (auch Jahre nach dem Durchbruch zum multinationalen Vertrieb ihrer Produkte oder Dienstleistungen) weiterhin durchschimmern zu lassen.

»Wir sind ein engagiertes Team aus Gesundheits- und Fitnessbegeisterten, die Produkte erstellen, die das Leben der Benutzer positiv verändern. «34

33 Quellen: http://corporate.withings.com/, www.fitbit.com/de/fitbit-wellness (zuletzt aufgerufen am 19.11.2015). Zu den Fitbit Wellness Kunden gehören neben vielen anderen inzwischen die Bank of America, IBM und die Time Warner Gruppe. Im April 2016 gab das Unternehmen Target bekannt, dass es 335.000 Fibit-Devices für seine Belegschaft geordert hatte. Das Unternehmen Barclays bot seinen Angestellten zur gleichen Zeit ca. 75.000 Tracker von Fibit an (vgl. Christl und Spiekermann 2016: 65).

34 Quelle: www.fitbit.com/de/company (zuletzt aufgerufen am 22.07.2015). Gary Wolf schreibt über zwei Gründungsmitglieder von Fitbit: »Park and his partner, Eric Friedman, first showed their prototype at a San Francisco business conference in the summer of 2008. Five weeks later, Park and Friedman [...] were flying back and forth to Singapore to arrange production.«Quelle: www.nytimes.com/2010/05/02/magazine/o2self-measurement-t.html?_r=2\&re $\mathrm{f}=$ magazine\&pagewanted=all (zuletzt aufgerufen am 22.07.2015). Inzwischen bedient Fibit annähernd $27 \%$ des Wearble-Marktes (vgl. Christl und Spiekermann 2016: 62). Dementsprechend ist das Unternehmen als globaler Inkubator und öffentlichkeitswirksamer Promotor bestimmter Definitionsweisen von Selbstvermessung bzw. damit verbundenen Selbstverständnissen im Diskurs vertreten und trägt durch eine standardisierte Produktpalette zu einer erleichterten praktischen Adaption des Self-Tracking in unterschiedlichen Cesellschaftsbereichen bei; Ideen und Legitimität bezieht das Unternehmen dabei allerdings zu einem Gutteil aus dem dezentralen Basissegment der Self-Tracking-Szene, dessen Popularisierung es gleichermaßen vorantreibt. 



\title{
6 Selbstvermessung und Betriebliches Gesundheitsmanagement ${ }^{1}$
}

\author{
»Wer sich schneller entspannt ist besser als \\ jemand der sich nicht so schnell entspannt. \\ Der aber immer noch besser ist als jemand \\ der sich überhaupt nicht entspannt. « \\ Aus: »Wettentspannen« von Peter Licht
}

Gegenwärtig ist $\mathrm{zu}$ beobachten, dass sich in vielen Unternehmen verschiedene Technologien zur zahlenbasierten Vermessung und Analyse der sog. Vitalfunktionen von Angestellten etablieren, welche den ohnehin breit ausgebauten Bestand an Controlling-Systemen um neue Formen der numerischen Selbstbeobachtung erweitern. Anders als Tracking-Systeme wie die digitalisierte Zeiterfassung oder die Protokollierung des Internet-Traffic, die schon lang zum Standardrepertoire vieler Unternehmen gehören, ${ }^{2}$ zielt das Self-Tracking in viel stärkerem Maße auf die Analyse täglicher Gewohnheiten sowie die körperliche und geistige Verfasstheit der Angestellten. Den prägnantesten Anwendungsfall stellt bislang die episodische oder dauerhafte Installation von Selbstvermessungssystemen im Rahmen des betrieblichen Gesundheitsmanagements dar. ${ }^{3}$ Zwei einfache Beispiele: Das Pro-

Für eine kürzer gefasste Auseinandersetzung mit den in diesem Kapitel verhandelten Problemen, siehe Mämecke (2016).

2 Derartige Technologien firmieren inzwischen unter dem Begriff »Digitalized Management Methods« (DMM) und wurden in den letzten Jahren z.B. durch Phoebe Moore aus der Perspektive des New Materialism untersucht (Moore 2017, 2018, Moore et al. 2018).

Die Programmatik des betrieblichen Cesundheitsmanagement soll gesundheitsförderndes Verhalten am Arbeitsplatz ermöglichen und bewirken. Es richtet sich in einem semantischen Zweischritt meist gleichzeitig an Angestellte und Personalentwicklung. Einerseits dient es der Prävention, zum anderen ist es aber auch eng mit dem Konzept der Resilienz verbunden, das sich sowohl als Variante des Präventionskonzeptes und als Alternative zur Prävention beschreiben lässt: »Während Prävention heute interveniert, damit morgen die befürchteten Schäden nicht eintreten, will Resilienzförderung heute dazu befähigen, die möglicherweise morgen eintretenden Schäden besser zu bewältigen « (Bröckling 2017: Online unter: https://s oziopolis.de/beobachten/kultur/artikel/resilienz/). Die Ansprüche der Resilienz sind entsprechend widersprüchlich; es animiert nicht nur dazu »vorhersehbare Belastungen abzufedern, 
gramm »Moove - Tu's für dich« der Firma Vitaliberty kombiniert verschiedene Activity-Tracker, Apps und Sensoren um über die Berechnung von Herzratenvariabilität und der täglich durch die Teilnehmer"innen zurückgelegten Schritte eine Bewertung über das Maß an körperlicher Aktivität sowie das Stresslevel der Angestellten eines Unternehmens vornehmen zu können. Die so erzeugten Daten werden über Cloud-Computing sowohl den Angestellten selbst zurückgespiegelt ${ }^{4}$ als auch in aggregierter und anonymisierter Form dem "gesamten Unternehmen« zugänglich gemacht:

»Aussagekräftige Vergleiche zwischen Mitarbeitergruppen können gezogen und Benchmarking betrieben werden. Sparen Sie sich Zeit und Geld für aufwändige Analysen - moove macht Risikopotenziale unmittelbar sichtbar. Übersichtliche Darstellung von Häufigkeitsverteilungen und Mittelwerten. $\ll^{5}$

Das Programm Evergreen, das wie Moove für den AOK-Gesundheitspreis für digitale Prävention 2013 nominiert war, soll neben der Selbsteinschätzung der eigenen Gesundheit zusätzlich externe medizinische Daten (z.B. der Hausärztin) aufnehmen. In beiden (so wie in den meisten übrigen) Fällen werden die so gewonnen Daten genutzt um "gesundheitshemmende Faktoren ${ }^{6}$ im Unternehmen frühzeitig zu identifizieren, welche die Produktivität mindern und zu hohen Folgekosten führen können. Darüber hinaus dienen sie als »Ausgangspunkt zur Ableitung von Maßnahmen «, ${ }^{7}$ wie der automatischen und individuellen Ermittlung der passenden gesundheitsfördernden Programme des Betriebes für die einzelnen Angestellten. Erreicht der Stresspegel einer Angestellten im Rahmen des Moove-Programms etwa ein bestimmtes Niveau, können mit Hilfe einer Smartphone-App Entspannungsübungen durchgeführt oder Gesundheitsberater via Telefonverbindung zugeschaltet werden - Althussers berühmtes Bild der Anrufung des Subjekts ist in diesem Zusammenhang damit beinahe wörtlich zu verstehen (vgl. Althusser 1977: 140ff.).

Die sensorische Überwachung von Vitalfunktionen konnte sich über derartige Programme während der letzten zwei Jahre Schritt für Schritt sowohl unter den

sondern auch auf unkalkulierbare Störungen vorbereitet zu sein« und vorbereiten »auf künftige Krisen und Katastrophen kann man sich immer - und sei es durch Achtsamkeitsmeditation« (Bröckling 2017: Online unter: https://soziopolis.de/beobachten/kultur/artikel/resili enz/).

4 »Die gemessenen Daten werden automatisch an das moove Gesundheitsportal übertragen, so hat der Mitarbeiter sein persönliches Stresslevel immer im Blick.«Quelle: https://www.co rporate-moove.de/home/ (zuletzt aufgerufen am 13.04.2015).

5 Quelle: https://www.corporate-moove.de/home/ (zuletzt aufgerufen am 13.04.2015).

6 Quelle: www.aok-bv.de/imperia/md/aokbv/mediathek/gg/spezial/gg_spezial_03_14_ansicht_ offset.pdf (zuletzt aufgerufen am 08.05.2015).

7 Quelle: https://www.corporate-moove.de/home/ (zuletzt aufgerufen am 13.04.2015). 
Belegschaften global agierender Konzerne als auch kleiner und mittelständischer Unternehmen etablieren. ${ }^{8}$ Zwar ist die Zahl der Unternehmen in denen solche Systeme zum Einsatz gebracht werden gegenwärtig noch vergleichsweise gering, ihre Zahl steigt jedoch kontinuierlich ${ }^{9}$ und mit ihr die Zahl der entsprechenden Beratungsfirmen sowie Hard- und Software-Hersteller. Self-Tracking-Devices zur Abfrage, Aufzeichnung und Analyse von akkumulierten Körperwerten oder Gefühlszuständen einzelner Belegschaften oder Kohorten ergänzen damit zunehmend das Instrumentarium der Personalentwicklung vieler Unternehmen.

\subsection{Interdiskursbeziehungen: Burnout und Self-Tracking in Unternehmen}

$\mathrm{Zu}$ den prägnantesten Bedarfen, Gefahren oder Dringlichkeiten, die die Etablierung von Selbstvermessungstechnologien im Kontext betrieblicher Angestelltenverhältnisse derzeit begleiten, zählen Problemdiagnosen wie demografischer Wandel und ein daraus resultierender Fachkräftemangel oder die Dezentralität moderner Arbeitsverhältnisse sowie stetig steigende Belastungen der Angestellten vor dem Hintergrund von erhöhtem Wettbewerbsdruck. ${ }^{10}$ In den Angebotsbeschreibungen und Selbstdarstellungen privater Dienstleistungsunternehmen und Softwarefirmen werden diese Prognosen, die oftmals ganz unterschiedlichen Quellen entstammen, meist hierarchisch zu einer sich schrittweise verstärkenden Argumentation aufgeschichtet, wobei Arbeitsausfälle durch steigende Belastungen als hinreichendes Argument für die Durchführung von Maßnahmen zur Prävention von Stress und psychischen Erkrankungen in Unternehmen instrumentalisiert, ${ }^{11}$ demografischer Wandel und Dezentralität darüber hinaus allerdings vor allem als Begründungen für die technische Ausrichtung der Maßnahmen herangezogen werden.

Z.B. Appirio, AXA, MIT, SAP. Auch Betriebskrankenkassen, die diese Verfahren unterstützen, verwenden Self-Tracking-Systeme innerhalb der eigenen Belegschaft. So z.B. die BKK Mobile Oil.

9 Bereits im Jahr 2014 startete der Wearable-Hersteller Fitbit ein ähnliches Programm für Unternehmen. Zu den Anwendern des Programms zählt u.a. das international tätige Energieunternehmen BP. In Deutschland zählen auch viele Betriebskrankenkassen zu den Anwendern von Self-Tracking-Systemen im Arbeitskontext.

10 »Demografischer Wandel, Fachkräftemangel, steigender Wettbewerbsdruck: Die heutige Arbeitswelt stellt Unternehmen und insbesondere die Personalarbeit vor zahlreiche Herausforderungen.«Quelle: https://www.corporate-moove.de/home/ (zuletzt aufgerufen am 13.04.2015).

11 »Vielen Beschäftigten fällt die Erfüllung der Anforderungen der heutigen Arbeitswelt zunehmend schwer: Arbeitnehmer sind gestresst, erschöpft und die Zahl der Arbeitsunfähigkeitstage aufgrund psychischer Erkrankungen wächst stetig." Quelle: https://www.corporate-mo ove.de/home/ (zuletzt aufgerufen am 13.04.2015). 
Das Monitoring der Angestellten, dessen Grundlage ihre eigene Vermessung bietet, soll so u.a. ein evidenzbasiertes Personalmanagement ermöglichen, das ein vorzeitiges Ausscheiden von Beschäftigten aufgrund von körperlichen oder psychischen Erkrankungen frühzeitig zu erkennen vermag und durch regelmäßige Screenings die allgemeine »Belastungssituation einer Organisation ${ }^{12}{ }^{2}$ z.B. im Zusammenhang mit der innerbetrieblichen Altersstruktur sichtbar machen kann. Die Dezentralität moderner Arbeitsverhältnisse begründet darüber hinaus den Einsatz mobiler Geräte oder Apps, welche die (Selbst)Überwachung, Analyse und Erhaltung der Gesundheit auch in zeitlich flexiblen und nicht notwendigerweise ortsgebundenen Arbeitskontexten ermöglichen und sie vom Interieur physikalischer Firmenniederlassungen unabhängig machen soll. ${ }^{13}$ Unternehmen und Selbstunternehmen sehen sich damit nicht nur mit vergleichbaren Problemen konfrontiert, sondern nutzen auch ähnliche Technologien um sie zu kontrollieren.

Selbstvermessungstechnologien heben sich von anderen präventiven Maßnahmen zwar gerade dadurch ab, dass sie ihre Apologie aus der selbstverstärkenden Logik von Sozialstatistiken beziehen, die gleichermaßen zahlenbasierte Problemdiagnosen, evidenzbasierte Entscheidungshilfen für Interventionsstrategien und eine kontinuierliche Evaluation von Ergebnissen versprechen, diskursiv fügen sich so erzeugte Zahlenaggregate jedoch erst in Verbindung mit sinnbildlichen Beschreibungen zu artikulierbaren Gefahrenszenarien, erstrebenswerten Zielvorstellungen oder Selbstverständnissen zusammen. Die gewählten Begriffe sind dabei keinesfalls willkürlich sondern immer gleichzeitiger Ausdruck interdiskursiver Verstrickung z.B. medizinischer, technischer, sozialstruktureller und psychopathologischer Semantiken, die sich als Rationalisierungsformen sowie sukzessiver Ermöglichung und Verunmöglichung von kommunikativen Anschlüssen zu einem Machtfeld aus Sinn- und Wissensproduktion verdichten, in dessen Zentrum die Herausbildung einer bestimmten Subjektform steht.

Susann Sontag hat in ihren vielbeachteten Essays zur Metaphorik von Krankheiten verschiedene Beispiele zum diskursiven Charakter populärer Krankheits-

12 Quelle: www.psyware.de/de/precire-health (zuletzt aufgerufen am 13.04.2015).

13 Derartige Vermessungsportale lassen sich daher als Strategie betrachten, Orientierungsbedürfnisse zu erfüllen, die im breiteren Kontext unternehmerischer Eigenverantwortung, Individualisierung aber auch der voranschreitenden Digitalisierung vieler Arbeitsbereiche und den damit einhergehenden Strukturveränderungen überhaupt erst entstehen. Wohingegen Stefan Selke das Self-Tracking im Untertitel des Sammelbands »Lifelogging « in die semantische Nähe zu disruptiven Technologien setzt (2016), also zu digitalen Innovationen, die gezielt auf die »Transformation von Produktionsabläufen, Unternehmensstrukturen und Marktbeziehungen « (Nachtwey und Staab 2016:2) ausgerichtet sind und damit traditionelle Organisationsformen in verschiedenen gesellschaftlichen Bereichen erodieren, erweist sich ein Self-Tracking-System wie RescueTime im Grunde eher als ein stabilisierender Faktor von Produktionsordnungen innerhalb einer sich rasant verändernden Arbeitswelt. 
bilder vorgelegt, die sehr detailreich zeigen, wie die Symbolik von Krankheiten gesellschaftlichen Problemlagen, Ängsten und Ressentiments zum Ausdruck verhilft und sich letztlich mit subjektivierender Wirkung gleichermaßen auf kranke und gesunde Menschen niederschlägt bzw. sie als kranke oder gesunde Menschen diskursiv erzeugt (Sontag 1981).

Als Umschlagspunkt von einer vornehmlich empathischen Thematisierung von Krankheiten zu einem Diskurs der Schuldzuweisung, der das kranke Individuum in die Verantwortung nimmt, identifiziert sie die Ablösung der Tuberkulose durch Krebs als diskursiv präsenteste Krankheitsform. Eine Krankheit die von Beginn an immer auch als eine Erkrankung des Willens galt (vgl. Sontag 1981: 74). Die Sprache zur Beschreibung von Krebs entwickelt sich eng an den ökonomischen Katastrophenszenarien der damaligen Zeit; er ist die Krankheit »des nichtregulierten, abnormen, zusammenhangslosen Wachstums« (Sontag 1981: 75). Krankheitsmetaphern und ihre diskursive Wirkkraft sind damit von prägenden Ordnungsverständnissen und gesellschaftlichen Normen kaum trennbar. Die für den Frühkapitalismus als unabdingbar erachtete rationale Beschränkung und rigide Kontrolle ökonomischer Prozesse lies den Krebs in der öffentlichen Wahrnehmung damit als Phänotyp von ungebändigter Begierde und einer inneren Rationalitätskrise erscheinen. Wie Sontags historische Untersuchungen nahelegen beschränkt sich der diskursive Stellenwert von Krankheitsmetaphern nicht auf vorangehende Jahrhunderte sondern spielt auch bei gleichzeitigem Aufkommen evidenzerzeugender Technologien wie der Sozialstatistik weiterhin eine wichtige Rolle bei der Konstruktion und Popularisierung von Notständen und Bedarfen, z.B. für die biopolitischen Interventionen der modernen Psychopathologie.

So auch bei der Einführung von Selbstvermessungstechnologien in Unternehmen, im Zuge derer vor allem auf stereotype Burnout-Szenarien rekurriert wird um die Gefahren von psychischem Stress zu thematisieren. ${ }^{14}$ Das bemerkenswerte an den Rekursen auf das Burnout-Szenario ist allerdings, dass sie sich nicht in der Instrumentalisierung eines abschreckenden Krankheitsbildes erschöpfen, sondern mit dem Burnout-Begriff eine umfangreiche Chronik diskursiver Auseinandersetzungen zu den Arbeitsverhältnissen postindustrieller Staaten in den speziellen Diskurs des betrieblichen Gesundheitsmanagement importieren. Eine Chronik die zu allererst davon zeugt, dass sich der Grundtenor des Burnout-Begriffs im Verlauf dieser Auseinandersetzungen mehrfach geändert und seine Bedeutung schließlich

14 Ein besonders aussagekräftiges Beispiel ist das »Burnout-Bingo« das die Firma Vitaliberty im Rahmen eines theaterpädagogischen Seminars unter dem Titel »Wenn die Akkus leer sind... Business-Balance zwischen Himmel und Hölle« auf dem Corporate Health Congress 2013 veranstaltet hat. Quelle: https://www.youtube.com/user/vitaliberty (zuletzt aufgerufen am 17.03.2014). 
ins Gegenteil verkehrt hat. Wohingegen der Begriff zunächst überwiegend verwendet wurde um subjektives Leidensempfinden unter den strukturellen Bedingungen $\mathrm{zu}$ hoher Arbeitsbelastungen zum Ausdruck zu bringen, dient er gegenwärtig vor allem der Artikulation volkswirtschaftlicher Defizite durch Burnout-bedingte Arbeitsausfälle die das Subjekt und seine Verpflichtung zur Selbstsorge in den Mittelpunkt stellt. So wird der Beginn der steilen Karriere des Burnout-Begriffs in den Jahren nach $2004^{15}$ vielfach gerade darin gesehen, dass er eine Möglichkeit eröffnet hat, im Kontext prekärer Arbeitsverhältnisse, in denen Positionen nicht dauerhaft zugeteilt, sondern in regelmäßigen Abständen immer wieder von neuem ausgehandelt werden müssen (vgl. Rosa 2009: 662) und in denen jeder Arbeitsausfall eine potentielle Gefährdung für den Marktwert des eigenen Arbeitskraftunternehmens darstellt (vgl. Voß et al. 1998), ${ }^{16}$ eine Erkrankung noch unter der Hervorhebung der eigenen Leistung artikulieren zu können. Denn wer ausgebrannt ist muss zunächst gebrannt haben (Neckel et al. 2013: 8). Damit wurde der erschöpfungsbedingte Arbeitsausfall in eine Rhetorik integriert, die im metaphorischen Sinne der Kleidsamkeit eines Verwundetenabzeichens entsprach (vgl. Schmidbauer 2010: 159). ${ }^{17}$

Mit der zunehmenden Popularisierung drückte der Begriff schließlich ein viel allgemeineres »Unbehagen am Leistungsdruck im heutigen Berufsleben, an der Beschleunigung von Arbeit und Kommunikation, an alltäglicher Überforderung und neu empfundenen Formen der Entfremdung « (Neckel et al. 2013: 7) aus. Burnout wurde damit der Status einer breit debattierten Pathologie verliehen, die nun nicht mehr nur einzelne Individuen betraf, sondern in viel allgemeinerer Weise da$\mathrm{zu}$ verwendet wurde die "psychische Seite« der destruktiven Wettbewerbsformen (Neckel et al. 2013: 17) gegenwärtiger ökonomischer Verhältnisse öffentlich zu thematisieren. Wer zu diesem Zeitpunkt »Burnout « sagte, sprach bereits im Modus der Kulturkritik (Bröckling 2013: 179).

15 Der Begriff reicht in die 1970er Jahren zurück und wurde von Herbert Freudenberger geprägt, der mit dem 1974 erschienenen Aufsatz »Staff burn-out«strukturelle gesellschaftliche Bedingungen als mögliche Ursachen für die emotionale und körperliche Erschöpfung seiner Sozialarbeitkolleg*innen diskutierte (Neckel et al. 2013: 10). Ein öffentlichkeitswirksamer Diskurs unter dem Begriff Burnout entfaltet sich allerdings erst in den Jahren nach 2000.

16 Alternative Modellierungen stellen z.B. der Mitarbeiter-Unternehmer (Deutschmann et al. 1995: 445) im Kontext der Theorie reflexiver Modernisierung sowie das Unternehmerische Selbst (Bröckling 2007) aus Gouvernementalitätstheoretisch-Diskursanalytischer Perspektive dar.

17 Womit natürlich nicht ausgesagt ist, dass damit auch eine tatsächliche Akzeptanz der Erkrankung oder gar eine Anerkennung der Leistung einherging, für die mit diesem Begriff zum Ausdruck gebracht werden soll. Psychische Erkrankungen und (vorübergehender Verlust der Arbeitsfähigkeit) führen auch in den wohlhabendsten (Post-)Industrienationen nicht selten zum Verlust der Anstellung oder sozialem Ausschluss. 
Wie bereits anhand des Begriffs der Depression gezeigt wurde, dessen gesellschaftliche Bedeutung Alain Ehrenberg genealogisch als Ausdruck eines Selbst untersucht hat, das erschöpft davon ist, ses selbst $<\mathrm{zu}$ sein, lässt sich eine diskursive Konjunktur von Verausgabungs-, Ermüdungs- und Überanstrengungsdiskursen in der jüngeren Geschichte postindustriell geprägter Staaten gerade dann beobachten, wenn disziplinarische Modelle der Verhaltenssteuerung durch die Verpflichtung zur persönlichen Initiative bei gleichzeitigem Gebot zur performativen Selbstverwirklichung ersetzt werden. Damit verbindet sich die Metaphorik von Erschöpfungssyndromen unmittelbar mit modernen Arbeitsverhältnissen sowie einem Verständnis zeitgenössischer Individualität und ihrer Dilemmata (Ehrenberg 2011: 14ff.). Schon in den 1980er Jahren wurde das moderne Unternehmen daher zum »Vorzimmer der nervösen Depression« ernannt (Ehrenberg 1991: 270f.) ${ }^{18}$, was psychosomatische Störungen, Angstzustände und Depressionen gleichsam auch zu leitenden Themen der Arbeitsmedizin ${ }^{19}$ und der sich entwickelnden Personalentwicklungsforschung machte. Die breitenwirksame Auseinandersetzung über die psychischen Zumutungen moderner Arbeitsverhältnisse wurde zu diesem Zeitpunkt durch pharmakologische, psychotherapeutische und sozialpolitische Positionen besetzt, die im depressiven Subjekt vor allem das Subjekt seiner eigenen Konflikte sahen und deren Programme daher auf die Herausbildung einer Individualität zielten, die selbstständig handeln und sich verändern kann, indem sie sich auf ihre inneren Antriebe stützt (Ehrenberg 2011: 299).

Ein vergleichbarer Paradigmenwechsel ist während der letzten Jahre im Zusammenhang mit dem öffentlichen Umgang zum Thema Burnout zu verzeichnen. Bei ansteigender Anerkennung als eine therapiebedürftige Krankheit durch verschiedene am Diskurs beteiligte Institutionen wird der Burnout in die Behandlungs- und Präventionsprogramme therapeutischer und beratender Agenturen integriert und dabei sein kulturkritischer Status im Diskurs minimiert. Mehr noch als bei der Depression offenbart im Grunde bereits die Definition des ICD-10-Index, der das Burnout-Syndrom erstmals aufgenommen hat, eine Verschiebung der Bedeutung des Begriffs, die sich auch über den medizinischen Fachdiskurs hinaus fortsetzt. Denn das Burnout-Syndrom wird dort nicht als psychische Erkrankung geführt, sondern ist unter dem Abschnitt »Problems

18 Zitiert nach Ehrenberg (2011: 245).

19 Foucault hat die Herausbildung der arbeitsmedizinischen Biopolitik in der zweiten Hälfte des 18. Jahrhunderts als Fokussierung auf»Endemien «beschrieben, die im Gegensatz zu Epidemien als permanente Faktoren »des Entzugs von Kräften, der Verminderung von Arbeitszeit, des Energieverlustes und ökonomischer Kosten« zu einer Verwaltung von Krankheiten gegenüber den Versuchen ihrer Ausrottung geführt haben (Foucault 2001 [1975-1976]: 287f.). Die sich hierin begründenden arbeitsmedizinischen Interventionsstrategien beziehen sich jedoch zu dieser Zeit nicht auf psychische Faktoren. 
related to life-management difficulty ${ }^{20}$ klassifiziert. Durch das ausdrückliche Ausklammern sozioökonomischer Faktoren negiert die medizinische Definition der Erkrankung damit gerade die gesellschaftlichen Problemlagen, für deren Artikulation der Begriff im öffentlichen Diskurs lange verwendet wurde und setzt das Syndrom in die semantische Nähe zu »unzulänglichen sozialen Fähigkeiten ${ }^{21}$ des Individuums.

Gesundheit wird im Burnout-Diskurs kongruent $\mathrm{zu}$ dem viel allgemeineren "pursuit of healthiness « (Greco 1993) daher inzwischen als Gegenstand und Resultat des eigenen Willens konstruiert. Eine derartige psychosoziale Konzeption von Gesundheit zielt nicht mehr auf die Beschreibung eines aktuellen Zustands, sondern wird zu einem Zeichen von Initiative, Anpassungsfähigkeit und Dynamik: Unausgewogene Ernährung oder zu wenig Bewegung stellen in dieser Hinsicht bereits selbst korrekturbedürftige Tatsachen dar, denn wenn »Selbstbeherrschung und Autonomie die Voraussetzung für Gesundheit sind, ist ein mangelnder Wille und eine unzureichende Selbstführung bereits das erste Symptom einer Krankheit, deren Ursache letztlich im Inneren des Subjekts liegt« (Lemke 2000: 40f.).

Anders als die Krankheitsmetaphern konventioneller medizinischer Programme, stellt der Burnout-Begriff damit nicht einfach einen Hebel zur zentralistischen Durchsetzung von Hygienemaßnahmen oder Ähnlichem dar. Denn weder akute noch chronische Erkrankungen zählen zu den ausgewiesenen Interventionszielen der Self-Tracking-Systeme betrieblicher Gesundheitsmaßnahmen. Vielmehr sind sie konzeptionell darauf abgestimmt »die persönlichen Stresswerte [der] Mitarbeiter $\mathrm{zu}$ optimieren und deren Stressbewältigungskompetenzen nachhaltig $\mathrm{zu}$ fördern ${ }^{22}$ indem sie ihnen in viel allgemeinerem Sinne die erforderlichen Mittel für eine Planung der eigenen Ressourcen, wie die Spiegelung der täglichen Bewegung oder aufgenommenen Kalorien bereitstellen. Eine perfide Besonderheit des Burnout-Begriffs liegt damit gerade darin, dass er heute unter dem Anschein eines gesundheitspolitischen Entgegenkommens zur technologiegestützten Regulierung des eigenen Verschleißes unter hoher Arbeitsbelastung animiert, um einem Krankheitsbild vorzubeugen, dessen diskursive Funktion ursprünglich gerade darin lag, die Pathologien der Wettbewerbsgesellschaft artikulierbar zu machen.

20 Quelle: http://apps.who.int/classifications/icd10/browse/2015/en\#/Z73.0 (zuletzt aufgerufen am 12.01.2016). ICD steht für Internationa Statistical Classification of Diseases and Related Health Problems). Es ist das zentrale Klassifikationskompendium der Weltgesundheitsorganisation (WHO).

21 Quelle: www.icd-code.de/suche/icd/code/Z73.html?sp=Sz73 (zuletzt aufgerufen am 12.01. 2016).

22 Quelle: https://www.corporate-moove.de/home/(zuletzt aufgerufen am 13.04.2015). 


\subsection{Interdiskursbeziehungen: Quantified Self und Self-Tracking in Unternehmen}

Programme der hier beschriebenen Art ließen sich auf rein formaler Ebene sicher leicht als rechtliche und institutionelle Inkubatoren von Selbststeuerungen skizzieren, denen ganz offenkundig Regierungsstrategien zu Grunde liegen, die in Form von Manualen, Empfehlungen, Verordnungen, Gesetzen und Strategiepapieren auch offen einsehbar sind. Allerdings blieben bei einer empirisch derart eng zentrierten Analyse die diskursiven Erscheinungsbedingungen und BottomUp-Effekte dieser Entwicklung zwangsläufig unterbelichtet.

Denn die Praktiken der Selbstvermessung werden in der Regel nicht in direkter Weise diktiert. Vielmehr werden sie Unternehmens-, Technologie- und Dienstleisterübergreifend als Maßnahmen im Sinne der Beschäftigten angeregt, wobei sie argumentativ durch eine Ambiguität aus programmatischer Gefahrenprävention und partizipativer Unternehmenskultur gerahmt werden.

Foucault beschreibt die Entstehung neuartiger Machttechnologien demgegenüber als die Summe einer »Vielzahl von oft geringfügigen, verschiedenartigen und verstreuten Prozessen, die sich überschneiden, wiederholen oder nachahmen, sich auf einander stützen, sich auf verschiedenen Gebieten durchsetzen, miteinander konvergieren - bis sich allmählich die Umrisse einer allgemeinen Methode abzeichnen« (1977: 177). Aus diskurstheoretischer Perspektive, die den Analysefokus nicht programmatisch auf den Bereich des praktischen Wissens aus Handlungsanleitungen, Manualen, Leitfäden, Richtlinien und Gesetzen beschränkt, stellt sich das Aufkommen von Selbstvermessungspraktiken in den Arbeitsalltag verschiedener Berufssparten damit vielmehr als produktive Konfrontation gegenläufiger Gesundheits-, Emanzipations-, und Technologiediskurse dar, die sich im Bereich der betrieblichen Selbstvermessung gegenwärtig zu funktionalen, dabei allerdings niemals abgeschlossenen Prozessen verdichten. Denn die Bedarfe, Notstände oder Nutzen, die den Empfehlungen oder Verordnungen zugrunde liegen und auch die Erscheinungsformen der Self-Tracking-Tools selbst, ihr Funktionsumfang oder die nahegelegten Nutzungsweisen sind selbst bereits diskursiv erarbeitet, werden kontinuierlich verändert oder (re)kombiniert und überwinden daher niemals den Status einer nur vorläufigen Stabilität. Als Kreuzungspunkt verschiedener Teildiskurse konstituieren sich die Anwendungsszenarien und Technologien des Selbstvermessungsfeldes vielmehr als Bündelungen von Wiederholungen, Regelhaftigkeiten und Wahrscheinlichkeiten, die nicht durch Subjekte produziert werden, sondern sich als Resultat der Ermöglichung und Verunmöglichung bestimmter Anschlüsse ergeben, an denen ganz unterschiedliche institutionelle oder nichtinstitutionelle, gegenwärtige und vergangene Diskurspositionen beteiligt sind. Dies macht alle beteiligten Individuen und Institutionen zu Produktionsorten dieser Technologien, 
verweigert ihnen aber den Status von Produzent*innen - die Urheber*innen von Manualen, Empfehlungen und Verordnungen eingeschlossen.

Zwar liegen aus gouvernementalitätstheoretischer Sicht die Verheißungen des alltagsdatengestützten Personalmanagements auf der Hand, dennoch wären zur Minderung und Prävention aktueller Gefahren aus dem Bereich psychischer und körperlicher Arbeitsüberlastung auch viele andere Maßnahmen und Programme vorstellbar, wenn nicht sogar naheliegender. Zudem stellt sich die Frage, aus welchem Grund sich derart umfassende Kontrollinstrumente überhaupt unter den Belegschaften etablieren lassen. Natürlich mag der zwanglose Zwang derartiger Programme, der eine Beteiligung an betrieblicher Leistungs- und Gesundheitsvermessung zwar als freiwillig in Aussicht stellt, sich dabei allerdings auf die Dynamik innerbetrieblicher Konkurrenz berufen kann, hinter der in letzter Konsequenz das Machtverhältnis der Lohnabhängigkeit steht, ein entscheidender Faktor der Durchsetzung von Selbstvermessungstechnologien in Betrieben sein, allein kann er allerdings nicht erklären, weshalb z.B. die tradierten Institutionen des Arbeitnehmer*innenschutzes die Einführung dieser Systeme kommentarlos begleiten anstatt vor ihnen zu warnen. ${ }^{23}$

Wie die folgenden Beispiele verdeutlichen erweckt die Art und Weise, wie diese betrieblichen Vermessungs-Technologien und -Praktiken im Diskurs auftauchen den Eindruck als seien sie durch die Belegschaften gemeinhin akzeptiert. Positionen wie die der (nicht über Gewerkschaften oder Unternehmens-PR vermittelten) Belegschaft kommen dabei allerdings im Diskurs garnicht vor oder gelangen zumindest zu keiner medial Wahrnehmbarkeit. Dies weist die angeblich hohe Akzeptabilität der Tracking-Systeme innerhalb von Angestelltenverhältnissen somit besonders deutlich als einseitig konstruiertes Narrativ aus, das kaum Schlüsse über die tatsächlichen Einstellungen der Angestellten zu diesem Thema zulässt. Dieses Narrativ hebt dabei vornehmlich darauf ab den Etablierungsprozess von technischen Verfahren für die Protokollierung und Analyse von Leistung und Gesundheit vor möglichen Kontroll- und Überwachungsvorwürfen zu immunisieren indem sie als Technologien dargestellt werden, die sich außerhalb der Betriebe in den Innovationsnetzen emanzipatorischer Subkulturen entwickelt haben.

Unter der Beschränkung auf spezifische Teilbereiche sowohl der betrieblichen Personalentwicklung als auch der progressiven Selbstverdatung lässt sich hier ein

Mit Blick auf die allgemeinere Frage nach der Etablierung von Gesundheitsmanagement in Unternehmen finden sich indes eher Befürworter unter den etablierten Arbeitsschutzinstitutionen. So sieht die Projektgruppe Betriebliches Gesundheitsmanagement (BCM) des Landesbezirkes Nord der Gewerkschaft Verdi »in dem Konzept des Betrieblichen Cesundheitsmanagements eine zwischen Unternehmensleitung und Beschäftigtenvertretung abzustimmende Strategie, die auf Verbesserungen für das Wohlbefinden der Beschäftigten und höhere Produktivität zielt«, ohne das Konzept weitreichender zu Rahmen oder auf spezifische Maßnahmen wie z.B. das vermehrt aufkommende Self-Tracking im Detail einzugehen. 
diskursiver Zirkelschluss hervorheben: Wohingegen die technologieaffinen Freelancer selbstständig Applikationen für das Home Office entwerfen, die eine auffällige Nähe zu den Zeiterfassungssystemen der Großraumbüros aufweisen und sich dabei sogar explizit auf die tayloristische Betriebsforschung beziehen, orientiert sich hier nun das in diesen Kreisen vielfach als restriktiv und autoritär angesehene Angestelltenverhältnis an den Überwachungs- und Disziplinartechnologien des unabhängigen Selbstunternehmens. ${ }^{24}$

Mit dem Wandel der Arbeitsformen hat sich nicht nur das Verhältnis von Leistung und Disziplin verschoben, sondern auch das Verhältnis zwischen Arbeit und Gesundheit. Die systematische Entgrenzung zwischen den traditionell getrennten Lebensbereichen Arbeit und Freizeit, die lokale Entwurzelung, Leistungsdruck und das gesteigerte Verantwortungsniveau scheinen auch an den Quantrepreneuren nicht spurlos vorbeizugehen, sondern sich im Gegenteil als konjunkturelle Berg- und Talfahrten ihrer Datenlandschaften abzubilden. Insofern verwundert es nicht, dass auch in diesem Bereich kontinuierlich neue experimentelle Technologie-Mashups aus Produktivitäts- und Gesundheitsmessung entstanden sind. Anders als z.B. das Experiment von Kyrill Potatov (vgl. Abschnitt III. 4.6) stehen bei diesen Versuchen allerdings nicht nur allgemeine psychische Zufriedenheit oder eine innere Erfülltheit mit der eigenen Arbeit im Zentrum, sondern die Indikation des Zusammenhangs von psychischer und leistungsbezogener Stabilität.

Die langjährige, vornehmlich dezentrale und diskursive Entwicklung dieser Tracking-Technologien und ihre Verbreitung über File-Sharing-Netzwerke und App-Stores ${ }^{25}$ gehen ihrer systematischen Etablierung innerhalb des betrieblichen Gesundheitsmanagements damit zeitlich voraus.

Und auch auf semantischer und metaphorischer Ebene sind hier schnell entsprechende Kongruenzen auszumachen. Ganz ähnlich wie der institutionalisierte Gesundheitsdiskurs dreht sich auch die Diskussion quantifizierender Selbstexperimente um behelfsmäßige Gemeinplätze wie Burnout, Stress und Bewegungsmangel. Nicht nur über populäre Quantified-Self-Mitglieder, wie Maggie Delano, ${ }^{26}$ die zum Thema Burnout-Prävention eine Session auf der Quantified Self Conference

(Selbst)überwachungssensoren dieser Art, die die Körperfunktionen im modernen Produktionsprozess in Echtzeit überwachen und immer nur ein Startup weit davon entfernt zu sein scheinen, sich ganz mit dem menschlichen Körper zu verbinden, attestierte schon Donna Haraway in ihrem Cyborgmanifest, dass sie den Alptraum des Taylorismus bald würden idyllisch erscheinen lassen (vgl. Haraway 1995: 34).

»Some apps were top-charting, reaching the 5th place among the most downloaded paid apps in the Netherlands. "Quelle: www.marcoaltini.com/ (zuletzt aufgerufen am 22.07.2015). Maggie Delano leitete zu diesem Thema eine Session auf der Quantified SelfConference 2015 in San Francisco. Quelle: http://quantifiedself.com/2014/12/tracking-recovery-process-learn-m aggie-delano-qs15/ (zuletzt aufgerufen am 02.12.2014). 
2015 leitete, den burnout-bezogenen Annual Report der Datenkünstlerin Lillian Karabaic, ${ }^{27}$ den Mitbegründer der Berliner Quantified-Self-Gruppe Marcel Rütschlin, der eine Burnout-Erkrankung als Initiationspunkt und ursprüngliche Motivation für die eigene Vermessung angibt, ${ }^{28}$ oder Rachel Kalmar, die 30 verschiedene Tracker simultan verwendet um unter anderem ein Frühwarnsystem für Depressionen und Burnout zu entwickeln ${ }^{29}$, verzweigt sich der Diskurs bis tief in die Kreise der gesundheits- und produktivitätsbezogenen Experimente der Self-Tracker*innen. ${ }^{30}$

So zählte die experimentelle Erprobung von Verfahren zur Messung psychosozialer Stresssymptome und der Präventionsmöglichkeiten stressbedingter Gesundheitsprobleme schon im Gründungsjahr $2007 \mathrm{zu}$ vielbesprochenen Themen bei Quantified Self. ${ }^{31}$ Und auch jüngere Threads des Quantified-Self-Forums bieten aufschlussreiche Einblicke in die diskursiven Aushandlungsprozesse sowie die dezentrale Entwicklung und Erprobung der technischen Erfassung von körper- und umweltbezogenen Stressindikatoren bzw. entsprechender Anwendungsszenarien und Dateninterpretationsverfahren innerhalb der Community.

Der Blog-Artikel »Life Stress ${ }^{32}$ von Altini veranschaulicht zudem sehr eindrücklich, wie die selbstexperimentelle Stressforschung mit der Produktivitätsmessung verbunden und zur Ursachenforschung eines Burnouts verwendet wird und zeigt nebenbei wie Beides zur diskursiven Selbstherstellung als leistungsstarkes und belastbares Subjekt verwendet wird. Altini verbindet verschiedene Leistungsnachwei-

27 Quelle: http://quantifiedself.com/?s=annual+report\&x=0\&y=0 (zuletzt abgerufen am 14.02. 2017).

28 Quelle: www.welt.de/vermischtes/article109557685/Die-digitale-Entbloessung-ist-ein-lukrati ver-Markt.html (zuletzt aufgerufen am 04.11.2013).

29 Quelle: http://allthingsd.com/20130923/the-extremely-quantified-self-meet-rachel-kalmar-w ho-wears-21-fitness-trackers-at-the-same-time-video/ (zuletzt aufgerufen am 14.02.2017).

30 Auch der an die RescueTime-Webpage angegliederte Blog befasst sich regelmäßig mit dem Thema Burnout und folgt dabei ähnlichen Mustern: »When our daily stresses become commonplace, we're at the risk of becomming burn out« Quelle: http://blog.rescuetime. com/burnout-syndrome-recovery/ (zuletzt aufgerufen am 25.10.2017). Die enge Verbindung zur Quantified-Self-Community weisen die Entwickler über die regelmäßige Teilnahme an Community-Events nach: „We're pretty active in local Quantified Self meetups for example. That's a great group of people to be around when you're exploring the analytical side of productivity.«Aus einem Interview mit Robby McDonell. Quelle: http://lifehacker.com/im-robbymacdonell-and-this-is-the-story-behind-rescu-1497037183 (zuletzt aufgerufen am 14.11.2017).

31 So z.B. in dem frühen Quantified-Self-Blog-Eintrag »Wrist-Device for Real Time Stress Tracking«des Quantified-Self-Mitbegründers Cary Wolf vom 16.10.2007. Quelle: http://quan tifiedself.com/2007/10/wristdevice-for-real-time-stre/(zuletzt aufgerufen am 13.04.2015).

32 »This post is about being an entrepreneur, a toolmaker and a datascientist« Quelle: http s://medium.com/@marco_alt/life-stress-47b9576do9e7\#.105e5053a (zuletzt aufgerufen am 15.04.2015). 
se, wie Produktivitätswerte und Angaben über die Qualität der aufgewendeten Arbeitszeit, mit einem anekdotischen Kontext. In kürzeren Texten beschreibt er, wie er, bald nachdem er Europa verlassen hatte, um eine Stelle in einem Startup in San Francisco anzutreten, Arbeitsstunden in Höhe von "16/day« bzw. "300+/month« aufwendete. Im Anschluss an eine einjährige Leistungsphase erlebte er mit dem Ende seines USA-Aufenthalts auch einen Einbruch seiner Produktivität.

»Life stress piled up, until the inevitable happened. My productivity crashed: I almost went down to the infamous 40 hours/week (or $\sim 160 /$ month). $\ll^{33}$

Um seinen Leistungseinbruch auf infame 40 Stunden pro Woche zu kompensieren, widmete er sich mit technischen Mitteln der Wiederherstellung des Arbeitsvermögens und der zukünftigen Vermeidung weiterer Leistungseinbrüche. Auch hier wird die Herzratenvariabilität erneut als psychisch-physiologischer Indikator von Stresssymptomen verwendet:

»Making a few oversimplifications, we expect HRV to be higher when we are in a better physiological state, less stressed, ready to perform [...]. Looking at my HRV [...], I could clearly see it was crashed during weeks I was not productive [...]. On the other hand, working many hours/day was typically associated with higher HRV. ${ }^{34}$

Altinis Selbstexperiment stellt dabei ein eindrückliches Beispiel für den anwendungsorientierten Pragmatismus dar, der als Versuch »Dinge zum Laufen zu bringen« (vgl. Knorr Cetina 2012: 24[Hervorhebung im Original]) die meisten SelfTracking-Projekte begleitet. Die Vorannahmen, die sich mit dem ansonsten weitestgehend atheoretischen Charakter selbstquantifizierender Forschungsprozesse verbinden, sind aus diskursanalytischer Perspektive dabei weitaus aufschlussreicher als die erhobenen Daten selbst. So lässt sich anhand der Schlussfolgerungen Altinis nicht nur in allgemeiner Weise die entscheidungsimprägnierende (vgl. Knorr-Cetina 2012: 28) Wirkung interpretativer Wissensbildung verdeutlichen, sondern auch deduktiv auf das Selbst- und Weltbild des interpretierenden Subjekts schließen. Dies verdeutlicht etwa der statistische Zusammenhang zu dem Altini die zwei getrennt (und anhand getrennter Self-Tracking-Technologien) gebildeten Deskriptionen über Stresslevel und Produktivität verbindet:

»There was a really strong correlation between my baseline HRV (7 days moving average) and more highly productive hours, as logged by RescueTime. ${ }^{35}$

33 Quelle: https://medium.com/@marco_alt/life-stress-47b9576do9e7\#.105e5053a (zuletzt aufgerufen am 15.04.2015).

34 Ebd.[Hervorhebungen im Original].

35 Quelle: https://medium.com/@marco_alt/life-stress-47b9576do9e7\#.105e5053a (zuletzt aufgerufen am 15.04.2015 [Hervorhebungen im Original]). 
Obgleich man diesen Zusammenhang in zwei Richtungen lesen kann, aus dem Zusammenhang also auch schließen könnte, dass der erhöhte Stress die Produktivität gemindert hat, entscheidet sich Altini für die Interpretation dernach das Stresslevel gestiegen ist, weil die Produktivität eingebrochen ist. Er separiert eine durch körperbezogene Indikatoren nicht beschreibbare Stresskategorie, die er als genuinen Bestandteil der von ihm gewählten Lebensweise essentialisiert.

»In today's society things can be very different. I was working many hours trying to build something I believe in, and that was definitely positive stress. $\ll^{36}$

Da wo eine Korrelation zwischen Lohnarbeit und der Vorstellung eines erfüllenden Lebens besteht, liefert die Produktivitätsmessung die Indikatoren für subjektives Wohlbefinden.

»RescueTime was my perfect indicator of wellbeing. «7 $^{37}$

Die Experimente und Vorträge des Organisators des Denver Quantified-SelfMeetups Paul LaFontaine zeigen auf ähnliche Weise auf, wie das stressbezogene Self-Tracking in persönliche Coping- und Resilienz-Strategien eingebunden wird. ${ }^{38}$ LaFontaine beschreibt etwa im Vortrag »Using Heart Rate Variability to Analyze Stress in Conversation« wie er 154 Gespräche mit Vorgesetzten (und »CoWorkers « als Vergleichsbasis) loggte, ${ }^{39}$ um seine Stressbewältigung in beruflichen Situationen und unter längerer Anspannung zu verbessern:

»Breathing tools, understanding how the physiology reacts when meeting with the Big Bosses and finding the balance between Fight/Flight and relaxation can improve both how you prepare, and how you ramp yourself down when in an intense situation. And these same tools allow a fast transition to a new environment where you can be productive as appropriate for the situation. $\ll^{40}$

36 Quelle: https://medium.com/@marco_alt/life-stress-47b9576do9e7\#.105e5053a (zuletzt aufgerufen am 15.04.2015).

37 Quelle: https://medium.com/@marco_alt/life-stress-47b9576do9e7\#.105e5053a (zuletzt aufgerufen am 15.04.2015 [Hervorhebungen im Original]).

38 »Among the many ways of measuring stress, heart rate variability - patterns of change in time between heartbeats - has emerged as a practical choice. Discuss current tools and challenges with one of the most knowledge HRV trackers in the QS community. «Aus dem Programm des Quantified Self Public Health Symposiums 2016 Quelle: http://quantifiedself.com/2016/02/2 015-quantified-self-public-health-symposium/ (zuletzt aufgerufen am 06.04.2018).

39 Quelle: http://quantifiedself.com/qs15/ (zuletzt aufgerufen am 02.02.2018).

40 Paul LaFontaine, »Tracking Fight or Flight at the Office.«Quelle: http://quantifiedself.com/201 5/05/tracking-fight-flight-office/ (zuletzt aufgerufen 14.02.2017) Fight/Flight bezieht sich hier auf das fight-or-flight-response-syndrom (Kampf-oder-Flucht-Syndrom), das in der Physiologie und Neurobiologie den plötzlichen Anstieg von Adrenalin als Stressreaktionen auf Gefahrensituationen beschreibt. Eine ganz ähnliche Methode hat der Self Tracker Justin Lawler 
Der bereits im Ruhestand lebende Mark Leavitt geht noch einen Schritt weiter. Vergleichbar mit Altini und LaFontaine, deren Umgang mit Stresssituationen oder psychischer Erschöpfung sich um Fragen des richtigen Variabilitätstrainings ${ }^{41}$ oder der Unterscheidung von positivem und negativem Stress drehen, sucht er nach Korrelationen zwischen HRV und Willenskraft, die sich in der Vermeidung von Bewegungsmangel und kontinuierlicher Analyse unter Beweis stellt:

»In this talk, Mark looks at how heart rate variability relates to his willpower. [...] Also, you will get a glimpse of his amazing customized workstation with pedals to keep him active, a split keyboard on the armrests to keep his knees free and builtin copper strips for measuring HRV. ${ }^{42}$

Wer die Willenskraft nicht allein aufbringen kann, kann über das Selbstdisziplinierungstool Beeminder - sowohl das schlechte Gewissen, als auch die Selbstbestrafung technologisch externalisieren und bequem kleine Geldbeträge von der Kreditkarte abbuchen lassen, insofern die selbstverordneten Yoga- und Meditationsübungen zu kurz kommen: »Willpower not needed « ${ }^{43}$

\section{Diskursive und Nicht-Diskursive Bedingungen der Etablierung von Self-Tracking in Unternehmen}

Für Unternehmen, die ihre Belegschaften im Rahmen betrieblicher Gesundheitsprogramme $\mathrm{zu}$ Selbstvermessung animieren, ist es daher ein Leichtes über den Import der intrinsisch motiviert erscheinenden Experimente der Quantified-Self-Community und ihrer speziellen Verbindung von Selbstdisziplin und Empowerment-Rhetorik, sowie einzelner Elemente des Burnout-Diskurses argumentativ eine schwer zu hintergehende Verschmelzung aus belegschaftsnaher partizipativer Unternehmenskultur und dringender Notwendigkeit zu erzeugen.

»Zazengo enables all organizations with a desire for social action to attract, inspire and empower their members with a revenue-generating, easy-to-use, shared

auf die Analyse seiner Körperwerte während eines Show\&Tell-Talks angewendet: »Another stress-related piece. Justin shows through data how his body responded to the stress of giving a talk about his lifelogging experiences at QSEU15 [Quantified Self Europe Conference 2015. Anmerkung Th. M.].«Quelle: http://quantifiedself.com/?s=stress\&x=0\&y=0 (zuletzt aufgerufen am 14.02.2017).

41 Seine Bezeichnung lautet »personal variability training.«Quelle: http://quantifiedself.com/2 015/05/tracking-fight-flight-office/ (zuletzt aufgerufen 14.02.2017).

42 Quelle: http://quantifiedself.com/2016/04/mark-leavitt-daily-hrv-measure-health-willpower/ (zuletzt aufgerufen am 05.06. 2016).

43 Die Nutzung von Beeminder ist zunächst kostenlos, das Programm bucht allerdings langsam steigende Beträge (zum Beispiel in Höhe von fünf oder zehn Dollar) von der hinterlegten Kreditkarte ab, insofern die Ziele nicht eingehalten werden. Beeminder Review: Quelle: htt ps://youtu.be/JZH6rOHeqig (zuletzt aufgerufen 14.02.2017). 
platform that provides project matching, tracking, action and impact across multiple projects and organizations. $\ll^{44}$

Viele Unternehmen setzen inzwischen auch in nicht-diskursiver Weise darauf, über die Gewährleistung der technischen Integrationsfähigkeit populärer Fitnessarmbänder, private und außerbetriebliche Selbstverdatungsambitionen aufzufangen und in die firmeneigene Dateninfrastruktur zu inkludieren. Viele Firmen richten die Etablierung selbsttragender Überwachungssysteme für Stress, Bewegung oder Leistung daher gezielt auf bereits vorhandene Selbstverdatungspraktiken ihrer Belegschaft aus oder entwickeln diese Systeme unmittelbar unter Einbeziehung der Mitarbeiter*innen und der dort vorhandenen Erfahrungen mit Selbstverdatungstechnologien weiter. Die Unternehmen rekurrieren hier auf den diskursiven Austauschprozessen des Self-Tracking-Diskurses, die die einzelnen Devices oder ihre Integration in bestehende Software-Systeme weniger als die Entscheidungen einer hierarchischen Organisation und mehr als das gemeinsame Produkt einer firmeninternen Community erscheinen lässt.

Das Projekt CloudFit der Firma Appirio lässt sich als aufschlussreiches Beispiel für entsprechende Versuche heranziehen, die diskursive Entwicklungs- und Anwendungskultur der Self-Tracking-Szene aufzugreifen und innerhalb firmeneigener Strategien zur Krankheitsprävention praktisch fortzusetzen. Im Rahmen eines Pilotprojekts investierte das global agierende Unternehmen in Absprache mit ihrem Versicherungspartner Anthems die Rückzahlung eines Überschussbetrags, der für firmeneigene Gesundheitsprogramme vorgesehenen war, in portable FitnessTracker des Herstellers Jawbone und lud 200 Mitglieder der Belegschaft zu ihrer experimentellen Anwendung ein. Der Anschluss der Tracker an das firmeneigene Computernetz wurde in der folgenden Zeit sowohl unter Mitarbeit des Geräteherstellers als auch einigen Teilnehmer*innen des Testprogramms selbst verwirklicht, die durch die Firmenleitung »autorisiert « wurden, sich im Rahmen ihrer regulären Arbeitszeit mit der Entwicklung einer sog. "prototype aggregation engine« zu beschäftigen. Die Engine sollte eine unmittelbare Integration der SelfTracking-Daten in die zugehörigen Diskussionsgruppen der intern verwendeten Arbeitsorganisationstechnologien wie Salesforce oder das entsprechende SocialNetworking-Plug-Inn Chatter ${ }^{45}$ ermöglichen. Über Chatter konnten sich auch alle

44 Quelle: www.indeed.com/cmp/Zazengo?from=reviews (zuletzt aufgerufen am 12.01.2016). Auch über Zazengo gibt es eine enge Verbindung zur Quantified-Self-Community. Im bereits zitierten Bericht von Gary Wolf»How to make money from this « über das Treffen »Quantified Self Buisness Models« entfällt ein großer Teil auf dieses Startup. Quelle: http://quantifiedsel f.com/2010/03/quantified-self-business-model/ (zuletzt aufgerufen am 12.01.2016).

45 Bei Chatter handelt es sich um ein soziales Netzwerk, das u.a. der Vernetzung global agierender, virtueller Unternehmen sowie der Förderung und Abschöpfung kooperativ generierten Wissens dient: »Bieten Sie allen Mitarbeitern ein Forum, in dem sie Erkenntnisse teilen und 
übrigen Mitarbeiter*innen in die Gestaltung des firmeneigenen Tracking-Systems einbringen und z.B. selbstinitiierte Umfragen zu einzelnen Aspekten des Projekts oder numerische Vergleiche der gemessenen Aktivität unter den Teilnehmenden anregen. Hier entschieden die Angestellten nach einigen technischen Problemen im ersten Jahr auch über den Wechsel der Hardware von Jawbone zu Fitbit. Kontextbedingte Machtasymmetrien, die den unternehmensinternen Entwicklungsprozess dieses Tracking-Systems in gleicher Weise durchziehen wie die materiellen Abhängigkeiten das freiwillige Selbstverbesserungsengagement der Angestellten, sind hinter dem Eventcharakter der öffentlichen Darstellung nur schwer zu erkennen oder vermischen sich mit der beispielhaften Nutzung durch repräsentative Projektfiguren; ganz im Sinne der unverfänglichen Rhetorik, wie sie aus den Show\&Tell-Vorträgen der Quantified-Self-Meetups bekannt ist:

»For me, that's important because I don't sleep well. My personal goal this year is to sleep more. It drives more productivity. $\ll^{46}$

Die experimentelle und technisch teils sehr niedrigschwellige Pionierkultur der Selbstvermessung reproduziert sich auf diesem Weg über verschiedene Hierarchieebenen hinweg sowohl im diskursiven wie auch im nicht-diskursiven Sinne innerhalb betrieblicher Anstellungsverhältnisse, wobei sich über das hohe Maß an Selektivität, mit der sich sprachliche und technische Elemente aktueller Selbstvermessungstrends innerhalb von Unternehmen fortsetzen, vor allem die in der Selbstvermessung bereits angelegte Logik unternehmerischer Selbstoptimierung weiter verstärkt (vgl. Abschnitt III. 5.3).

\subsection{Belastung und Anpassung - Partizipative Approximation nachhaltiger Leistungsfähigkeit}

Bei näherer Betrachtung der medialen Oberflächen vieler portabler Stress- und Activity-Tracker, die am Arbeitsplatz zum Einsatz kommen wird sehr deutlich, dass sich die Erfolge und Misserfolge der eigenen Verhaltenskonditionierung, die über Software-Dashboards oder Weekly Reports ausgegeben werden, meist in Form von Balken- und Kurvendiagrammen darstellen. Vergleichbar mit den Produktivitätsmittelwerten bei RescueTime operationalisieren diese Technologien hier nun in Bezug auf gesundheitliche Präventionskategorien den Anschluss an spezifische Nutzer*innengruppen, Unternehmensbelegschaften oder einzelne Abteilungen.

neue Ideen vorschlagen können.«Quelle: www.salesforce.com/de/chatter/overview/ (zuletzt aufgerufen am 25.09.2015).

46 Chris Barbin, CEO bei Appirio. Quelle: www.citeworld.com/article/2115575/consumerization/w hy-appirio-issued-fitness-monitors-employees.html (zuletzt aufgerufen am 21.04.2015). 
Flexible Mittelwerte und hierarchische Rankings als Orientierungs- und Anreizstrukturen lassen die aufgewendeten Anstrengungen der Self-Tracker*innen als Anlageertrag erscheinen. Nicht nur in den Aussagen der beteiligten Dienstler*innen liegt der »Return on Prevention « hier nahe am »Return on Investment. « ${ }^{47}$ Diese Formen der systematisierenden Selbstbeobachtung basieren damit in elementarer Weise darauf, das Selbstverständnis unmittelbar mit dem Verständnis von Erfolgsfaktoren und dem eigenen Marktwert oder dem eigenen Wert für das Unternehmen $\mathrm{zu}$ verweben, ${ }^{48}$ um ganz im Sinne eines betriebswirtschaftlichen Benchmarking-Verfahrens Verbesserungs- bzw. Steigerungsmöglichkeiten zu identifizieren. Am Beispiel der technischen Verkettung von Appirios CloudFit mit der kommerziellen Kundenbetreuungs- und Provisionsmanagementsoftware Salesforce, die seit 2011 auch Funktionen für das sog. »Human-CapitalManagement « umfasst, oder den Rhetoriken der modernen Personalentwicklung, die durch Tracking-Technologien unterschiedslos auf »die Leistungsfähigkeit und Gesundheit von Unternehmen und die Menschen, die darin arbeiten ${ }^{49}$ zielt, lässt sich die Indifferenz verdeutlichen, in der hier personelle Gesundheit und Unternehmensvitalität verschmelzen. Durch die standardisierte Erhebung vordefinierter Indikatoren und die Verschaltung der Daten zu überindividuellen Vergleichen wird die mediale Repräsentation der eigenen Gesundheit damit als eine kalkulierbare Größe für den innerbetrieblichen Wettbewerb modelliert.

Das Programm Moove von Vitaliberty geht noch weiter und zeigt auf dem Software-Dashboard den wöchentlichen Aktivitätsdurchschnitt als Kennwert im unmittelbaren Vergleich zu allen übrigen Teilnehmenden des Unternehmens an: "Activities 6. +2.0 more then others. $\ll^{50}$

Wie schon Deleuze dezidiert herausstellte, setzt das moderne Unternehmen in Abgrenzung zu den Kontrollmechanismen der fordistischen Einschließungsver-

47 Thorsten Grießler »Leiter Vertrieb und Partnermanagement Vitaliberty $\mathrm{GmbH}$.«Quelle: http s://www.youtube.com/watch?v=PO3R8CXdFHw (zuletzt aufgerufen am 13.04.2015).

48 Moldaschl und Sauer beschreiben derartige Effekte bereits früh als Reaktion einer »Verinnerlichung des Marktes« auf Unternehmensebene, welche die Arbeitenden zunehmend in direkter Weise mit dem Markt kurzschließt und somit konventionelle Organisationsaufgaben wie Planung und Überwachung als »Externalisierung nach Innen« an die Angestellten überträgt. Dieser Argumentation folgend findet die »Unterwerfung (Subjektivierung im Sinne von Foucault) der Arbeitenden unter fremdgesetzte »ökonomische Zwänge« [...] so gesehen also nicht mehr im Arbeitsinhalt statt, wie es die Abstraktifizierungsthese der jüngeren Frankfurter Schule verkündet, sondern vielmehr im Verhältnis der Arbeitenden zu sich selbst« (Moldaschl et al. 2000: 220).

49 Aus der Beschreibung des Vortrags »People Analytics - Work Privacy Balance« der Personalentwicklerin und »Prozessgestalterin« Cornelia Reindl im Rahmen der re:publica 2015. Quelle: https://re-publica.de/session/people-analytics-work-privacy-balance (zuletzt aufgerufen am 29.09.2015). 
hältnisse vornehmlich auf eine »unhintergehbare Rivalität als heilsamen Wetteifer und ausgezeichnete Motivation, die die Individuen zueinander in Gegensatz bringt« (Deleuze 1993: 257). Analog lässt sich auch die Verbindung von individuellem Tracking mit kompetitiven Datenvergleichen innerhalb der Belegschaft als ein neuer Modus deuten, indem innerbetriebliche Konkurrenz als strukturierendes Organisationsprinzip der Unternehmensfitness aktiviert wird:

»Appirio is working on integrating corporate data into the dashboards so that it can organize teams around the company's structure. That would allow it to set up competitions between work groups or between offices in different regions of the world. $\ll^{51}$

Auch im Pharmakonzern Novartis zählt »ein gesunder Wettbewerb unter den Mitarbeitern« ebenso zu einem festen Bestandteil der Unternehmenskultur, wie die Verwendung von Withings-Pulse-Trackern im Rahmen der betrieblichen Krankheitspräventionsprogramme. ${ }^{52}$

Kongruent $\mathrm{zu}$ den durch Foucault herausgearbeiteten Erfassungstechniken, vermitteln auch diese digitalen Management-Technologien Leistungen oder Verhaltensweisen mit einer Grundgesamtheit, die sowohl als Vergleichsfeld sowie als Differenzierungsraum wirkt und die Individuen »quantifiziert und in Werte hierarchisiert (Foucault 1977: 236). Obgleich die Methodik der disziplinarischen Arbeitsorganisation somit hinter der Empowerment-Semantik des betrieblichen Gesundheitsmanagements hervor schimmert, ${ }^{53}$ handelt es sich bei ihr nicht

$51 \quad$ Chris Barbin im Interview mit dem Blog CiteWorld (Consumerization of IT in the enterprise). Weiter heißt es dort: »For now, workers can monitor their activity and sleep data through the standard Jawbone dashboard. There, they can create teams and comment on each other's progress. They can sort data about their team to look at who logged the most activity or had the best sleep.«Quelle: www.citeworld.com/article/2115575/consumerization/why-appirio-iss ued-fitness-monitors-employees.html (zuletzt aufgerufen am 21.04.2015). Das Unternehmen folgt dabei ebenfalls den aktuellen Trends des Quantified-Self-Diskurses und beabsichtigt in naher Zukunft auch GPS-Daten, sowie Funktionen zur Überwachung der Herzratenvariabilität mit aufzunehmen.

52 Dort heißt es weiter: »Durch die Einführung eines innovativen digitalen Programms in Verbindung mit der Tätigkeit von Gesundheitsprofis wurden sich alle Teilnehmer bewusst, wie wichtig es ist, sich körperlich zu betätigen und in einem gesunden Wettstreit den Teamgeist zu stärken.«Quelle: www.withings.com/de/solutions/professional-care.html (zuletzt aufgerufen am 10.04.2015. Inzwischen nur noch abrufbar über Waybayk-Maschinen). Auch das Unternehmen Withings selbst bewirbt sein Angebot unter Berufung auf kompetitive Aspekte des Programms: »Koordinieren Sie Einzel- und Teamwettbewerbe«; »Inspirieren Sie soziale Netzwerke. Teilnehmer können ihre Kollegen anfeuern, ausbuhen und ihnen personalisierte Nachrichten schicken.«Quelle: http://corporate.withings.com/(zuletzt aufgerufen am 19.11.2015).

53 Auch der Resilienzbegriff, der eng mit dem BCM verbunden ist, verweist mit einem Teil seiner Logik und Semantik auf die tayloristische Betriebswissenschaft und ihre maschinistischen 
einfach um ein hölzernes Pferd, durch das die rigiden Prinzipien der disziplinarischen Kontrolle an den Arbeitsplatz der Gegenwart transportiert werden, sondern um neuartige Kombinationen betrieblicher Steuerungsstrategien und dezentral entwickelter Innovationen technologieenthusiastischer Freelancer.

Disziplin, Optimierung qua Wettbewerb und ein aktivierender, libertärer Paternalismus bilden so ein Verhältnis funktionaler Ergänzung aus Prinzipien der Selbst- und Fremdsteuerung. Diese quantifizierenden Präventions- und Resilienzprogramme setzen dabei auf die Autonomie ihrer Teilnehmer (»Employees are selfdirected, so it's easy to administer $\ll),{ }^{54}$ sprechen sie z.B. im Verbund mit den Führungsmethoden der Output-Steuerung als freie Unternehmer*innen ihrer Selbst an, wechseln diesen Modus allerdings, wenn sie konstitutive Rationalitätsschwächen erkennen, die nicht durch Selbststeuerung kompensiert werden und adressieren die Selbstunternehmer*innen dann als Angestellte ${ }^{55}$ :

»Zazengo's key tool is the »Actvertisement«, - this is a message delivered to some targeted subset of employees, usually through the company intranet. ${ }^{56}$

Die Gestaltung der Soll- und Normwerte der Erfassungstechniken geben sie dabei ebenfalls nicht aus der Hand. Anstelle der Ausrichtung auf ein Normalfeld tendieren diese Technologien jetzt aber vielmehr zur Förderung kontingenter Konkurrenzbeziehungen, die ein permanentes individuelles Neujustieren notwendig machen. Dass die einzelnen "Qualitäten ausschließlich relational zu jenen der Mitbewerber zu bestimmen sind, verleiht dem Handeln den Charakter eines sportlichen Wettkampfs« (Bröckling 2012: 138). Verfahren wie diese bewegen sich damit in der ständigen diskursiven Ambivalenz von gemeinschaftlich erzeugten Vereinzelungstechnologien, die die Mitglieder (ob nun einer imaginierten Community oder formalen Belegschaft) separiert und unter Betonung ihrer Gegensätze wieder in Beziehung setzt.

Cesundheitskonzepte: »Der Begriff stammt ursprünglich aus der Werkstoffphysik und bezeichnet dort die Eigenschaft elastischer Materialien, nach Verformung wieder in ihre Ausgangsposition zurückzukehren. Überträgt man diese Vorstellung auf biologische, technische, soziale oder ökologische Systeme, bedeutet Resilienz das Oszillieren um einen Gleichgewichtszustand, den ein System im Fall von Abweichungen aus eigener Kraft durch geeignete Adaptionen wiederherzustellen sucht - das Prinzip der Homöostase. In einem weiteren Sinne beschreibt Resilienz das Vermögen eines Systems, Störungen und andere Stressoren $z u$ absorbieren oder ihnen standzuhalten, ohne einen »Regimewechsel« zu vollziehen, das heißt unter Aufrechterhaltung seiner grundlegenden Struktur und Funktionen« (Bröckling 2017: Online unter: https://soziopolis.de/beobachten/kultur/artikel/resilienz/). Quelle: https://www.humana.com/vitality (zuletzt aufgerufen am 14.03.2015).

55 Vgl. Bröckling (2015: Online unter: https://soziopolis.de/beobachten/kultur/artikel/gesteigert e-tauglichkeit-vertiefte-unterwerfung/). 
Dieser Modus entspricht dabei durchaus den paradoxen Anrufungen moderner Arbeitsverhältnisse die zwar die Bildung von Teams und Projektgruppen verlangen, letztlich aber jedes (unternehmerische) Individuum einzeln beurteilen.

In diesem »Diktat des Komparativ, dessen Vergleichspunkte und Maßstäbe sich fortwährend ändern«, können auch die Spitzenposition immer nur temporär besetzt werden. »Die Optimierung im Zeichen des Wettbewerbs installiert deshalb Feedbackschleifen und Technologien des (Selbst-)Monitorings, die kontinuierliche Anpassungen an sich ebenfalls kontinuierlich wandelnde Zielmarken ermöglichen sollen. Bei der Disziplinierung kann man Fortschritte erzielen, unter den Bedingungen des Wettbewerbs muss [aber] selbst der Beste das nächste Ranking fürchten. $\ll^{57}$

\subsection{Self-Tracking als Teil einer kompetetiven Selbstsorgekultur}

Im Verschränkungsverhältnis aus Burnout- und Quantified-Self-Diskurs stellt sich Self-Tracking am Arbeitsplatz somit in erster Linie als intrinsische Reproduktion jener zeitgenössischen Paradigmen dar, die das strukturelle Problem der Arbeitsüberlastung als ein persönliches Problem der Work-Life-Balance neu entwerfen, es in den Bereich individueller Verantwortlichkeit verschieben und es dem Subjekt dabei selbst überlassen Technologien für die eigene Kontrolle zu entwerfen und anzuwenden. ${ }^{58}$

Über die Affirmation des vormals gesellschaftskritischen Burnout-Diskurses durch die moderne Personalentwicklung (sowie Beratungs- und Softwaredienstleister), als auch über das unternehmerische Selbstverwirklichungsprojekt der gesundheitsbezogenen Selbstvermessung der Quantified-Self-Community, entwickeln sich Self-Tracking-Systeme im Rahmen des betrieblichen Gesundheitsmanagements so $\mathrm{zu}$ ambivalenten Kontrolltechnologien, deren Etablierung in starkem Maße auf außerbetrieblichen und privaten Selbstverdatungsambitionen und der allgemein zunehmenden Verbreitung von Produktivitäts- und ActivityTrackern basiert.

Unternehmen versprechen sich von diesen Technologien zum einen ein Personalmanagement in Echtzeit, das auf der Grundlage der Vitaldaten ihrer Angestell-

57 Vgl. Bröckling (2015: Online unter: https://soziopolis.de/beobachten/kultur/artikel/gesteigert e-tauglichkeit-vertiefte-unterwerfung/).

58 Siehe dazu exemplarisch den Eintrag »How Tracking What I Do Every Day Helped Me Find Better Work-Life Balance« der Self-Trackerin Melanie Pinola, unter der Rubrik »Productivity « auf der Webpage www.lifehacker.com. Quelle: http://lifehacker.com/how-tracking-what-i-do -every-day-helped-me-find-better-1710608636 (zuletzt aufgerufen am 28.07.2015). 
ten basiert, ${ }^{59}$ darüber hinaus sollen diese Technologien die Angestellten aber vor allem selbst in die Lage versetzen zwischen maximaler Arbeitsleistung und minimaler krankheitsbedingter Ausfallwahrscheinlichkeit annäherungsweise ein stabiles Verhältnis herzustellen, wobei im Gegensatz zu Leistungsdruck und Produktivitätsniveau diskursiv natürlich allein das Maß der persönlichen Krankheitsvorsorge als veränderbare Variable erscheint. D.h. das Maß an Belastungen selbst wird im Diskurs gar nicht oder als unveränderlich behandelt.

Das Aufkommen von Self-Tracking-Systemen am Arbeitsplatz, bzw. die Verschaltung der Vitaldaten von Angestellten zu numerischen Aggregaten und offen einsehbaren Durchschnitten, stellt sich aus dieser Perspektive als die Herausbildung einer kompetetiven Selbstsorgekultur dar, die im Verschränkungsverhältnis aus progressiven Technologie-Communities und betrieblichem Gesundheitsmanagement ihre Form gewinnt. Die Zielfolie dieser Selbstvermessungstechnologien ist ein Subjekt, das sich in der zahlenförmigen Symbolik betriebswirtschaftlicher Wettbewerbsanalysen selbst über die Kosten und Nutzen gewahr werden kann die es für ein Unternehmen bedeutet und das aus eigenem Antrieb den Widerspruch zwischen maximaler Arbeitsleistung und dauerhafter Erhaltung der Arbeitsfähigkeit approximativ als Teil einer technologiegestützten Personalentwicklung zu lösen versucht.

Das Leitbild des freien aber im betriebswirtschaftlichen Sinne rational handlungsfähigen Subjekts aktualisiert sich so in der diskursiven Auseinandersetzung zwischen Unternehmen, gesundheitspolitischen Institutionen und progressiven Self-Tracker*innen auch in der Form der Angestellten. Dabei ist über die tatsächlichen (in vielen Fällen sicher Weise konträr liegenden) Wünsche und Bedürfnisse der Angestellten wenig ausgesagt. Vielmehr verdeckt das auf Freiwilligkeit basierende Narrativ, dass eine intrinsische Motivation zum Experimentieren mit Technologien zur leistungs- und Gesundheitsüberwachung voraussetzt und die strukturellen Zwänge die mit Anstellungsverhältnissen jeglicher Art verbunden sind dabei überdeckt.

Die kalkulatorische Logik und der Datenhunger dieser Selbstvermessungssysteme machen allerdings bei den Angestellten selbst keinen Halt. TrackingProgramme wie das der Firma bwell, das der Selbstbeschreibung nach darauf spezialisiert ist, Organisationen anhand mobiler Fitnesstracker wie Fitbit oder Jawbone eine Errechnung der Bruttoerträge ihrer Mitarbeiter*innen im Zusammenhang mit ihrem Ausfallrisiko durch Krankheit zu ermöglichen, berücksichtigen inzwischen auch, dass die Produktivkraft einer Angestellten nicht kongruent mit ihrer physischen Entität berechnet werden kann, sondern sich nicht zuletzt durch die Reproduktionsarbeit ihres unmittelbaren Lebensumfeldes bemisst. Vor

59 Z.B. nach Maßgabe des u.a. unter den Abschnitten III. 6f. bereits näher beschriebenen Programms Moove von Vitaliberty. 
dem Hintergrund der ebenfalls im Wandel befindlichen Familienverhältnisse, die den fordistischen >Deak der Care-Arbeit zunehmend herausfordern, ist es daher naheliegend, dass für eine Bemessung der Produktivität als flexibles Verhältnis aus Leistungsertrag und Erhaltungskosten der Arbeitsfähigkeit, nicht nur Daten über die Reproduktionszeit der Arbeitenden selbst, sondern aller an dieser Reproduktionsarbeit beteiligten Personen relevant werden.

In Form eines kurzen Ausblicks soll sich im Folgenden nun noch einigen Beispielen gewidmet werden die sehr deutlich zeigen, dass auch auf diesem Gebiet derzeit in unterschiedlicher Weise experimentiert wird und die Grenzen des machbaren bzw. die Grenzen der Akzeptabilität systematisch ausgelotet und verschoben werden. Wie sich im Fall der Firma bwell bereits abzeichnet, ist daher in einem nächsten Schritt mit einer Ausdehnung des kalkulatorischen Zugriffs auf den Alltag der Angestellten zu rechnen, der potentiell auch das unmittelbare soziale Lebensumfeld wie Lebenspartner*innen und Kinder miteinschließt:

»Employees can enroll their spouses and children in the program, extending the benefits to the entire family. ${ }^{60}$

Parallel orientiert sich auch die wachsende Produktpalette der Wearables an Eltern und ihren Kindern.

»Cetting your young ones a Fitbit is a great way to help them take control of their fitness goals and see progress in real time. [...] Fitbit's forthcoming Ace tracker is basically an Alta made kid-friendly. It features the same iconic design, but in the Fitbit app, you'll set up a family account, which has animations, goal celebrations, and information geared toward children. $\ll^{61}$

»Keep the family connected in the app. ${ }^{62}$

60 Quelle: www.bwellamerica.com/how-bwell-solution-works (zuletzt aufgerufen am 21.04.2015). Weitere Beispiele für diese Entwicklung stellen etwa ein Eintrag des WellnessPoints-Katalogs des Life-Benefits-Programms der Firma BP oder des (mitunter Trackingbasierten) Wellness-Programms Vitality von Humana Insurance dar: »Working together or compete - but make it a team effort! Challange your coworkers, family, and friends to step up their step count. A little friendly competition can add spice to your routine!« Quelle: $h$ ttp://hr.bpglobal.com/LifeBenefits/Sites/Core/BP-Life-benefits.aspx (zuletzt aufgerufen am 14.03.2015). »As a family, parents and kids can set goals and earn rewards together « Quelle: https://www.humana.com/vitality (zuletzt aufgerufen am 14.03.2015).

61 Quelle: https://www.imore.com/which-fitbits-are-best-kids (zuletzt aufgerufen am 10.06. 2018).

62 Quelle: https://www.fitbit.com/ace (zuletzt aufgerufen am 10.06.2018). 
Technologien dieser Art sind damit gleichzeitig als eine sensorische Exploration von Alltagsroutinen, sowie als ein Modus anzusehen, durch die sich eine biopolitische Präventionspädagogik in die Mikrokosmen der modernen Familie verzweigt.

»Ace motivates kids to reach the 60 minutes of daily activity recommended by the CDC. $\ll^{63}$

Die numerische Information über Aktivitäts- und Ruhephasen von Kindern richtet sich in erster Instanz an deren Eltern, die Information darüber, wie die Eltern mit diesen Informationen umgehen, wird - so darf jedenfalls vermutet werden allerdings noch an anderer Stelle ausgewertet. ${ }^{64}$

»Cood sleep habits play a critical role in kids' health and well-being. Fitbit Ace let you know if they're getting the rest they need. $\ll^{65}$

Aber auch den Kindern selbst werden die Daten über ihr eigenes Verhalten in einer visuell vereinfachten Version dargeboten. Kongruent zu den Wettbewerbsimplikationen der >Erwachsenen-Tracker < beinhalten auch sie die Möglichkeit sich mit anderen Jungquantrepreneuren zu messen. Vereinfacht werden nicht nur die Rezeption von Aktivitätswerten und der kompetetiven Vergleiche, sondern auch weitere Planungs- und Management-Technologien. Die Octopus-Watch von Joy soll Kinder nicht nur an den Umgang mit numerischen Zeitkonventionen, Kalender- und Datumsformaten gewöhnen, sondern auch dabei helfen Termine einzuhalten, sowie Unabhängigkeit und Selbstwert zu erlangen.

»Kids can go head-to-head with others in step challenges and compete to win virtual trophies. ${ }^{66}$

»Octopus by JOY is the first icon-based watch that empowers kids by teaching good habits and the concept of time. [...] It fosters responsibility, independence and selfesteem. ${ }^{67}$

63 Quelle: https://www.fitbit.com/ace (zuletzt aufgerufen am 10.06.2018).

64 Der Funktionsumfang der Wearables ist sehr unterschiedlich. Ein Beispiel mit überdurchschnittlich vielen Features stellt die QQ-Watch von Tencent dar: »All-in-one wearable phone, Wifi GPS locator, fitness tracker, and more! «»When calling the Watch as a Listener, the QQ Watch will open up audio so you can listen to child's surroundings« Quelle: http://qqwatch.c o/ (zuletzt aufgerufen am 10.06.2018). Tencent ist als Dienstleister aus dem Bereich Onlinehandel, Onlinewerbung und soziale Internet-Medien u.a. an dem landesweiten chinesischen Citizen-Scoring-Projekt Sesame Credit beteiligt.

65 Quelle: https://www.fitbit.com/ace (zuletzt aufgerufen am 10.06.2018).

66 Ebd.

67 Quelle: https://www.heyjoy.io/ (zuletzt aufgerufen am 10.06.2018). 


\subsection{Interdiskursbeziehungen: Self-Tracking und partizipatives Risikomanagement im Gesundheitswesen}

Auch in Deutschland bieten immer mehr global agierende private Versicherungsdienstleister und gesetzliche Krankenkassen spezielle Präventionsprogramme oder Versicherungstarife an, die in ähnlicher Weise auf Freiwilligkeit basierende Systeme installieren, um ein direktes Beobachten und Antizipieren der positiven und negativen Effekte des eigenen Verhaltens innerhalb eines definierten Regelkanons zu ermöglichen. Die passiven Tracker wie Schrittzähler oder Sensoren für die Aufzeichnung der Herzratenvariabilität werden hier mehr und mehr durch sog. unstrukturierte Daten ergänzt, die eine aktive Eingabe der Vermessen(d)en erfordern und diese in flexible Versicherungstarife oder Bonusprogramme integriert.

Die hier vorherrschenden Gefahrenszenarien setzen meist bei sehr viel allgemeineren Bildern an, die auch über den speziellen Kontext der Arbeitsüberlastung hinaus anschlussfähig sind, dabei allerdings ihre Verbindung zu den Symptomatiken halten, die als spezifische Probleme der postindustriellen Wissensgesellschaft gelten: Allen voran der Bewegungsmangel, der durch Krankenkassen Mantra artig beschworen wird ${ }^{68}$ und der inzwischen durch die Verkaufsstrategien der Hardwarehersteller diskursiv lebendig gehalten wird:

"Sitting is the new cancer. ${ }^{69}$

Auszug aus dem durch Wearables unterstützten Bonusprogramm Gesundheits-Coaching der Techniker Krankenkasse: »Wer regelmäßig körperlich aktiv ist, leistet einen wichtigen Beitrag für die Erhaltung und Verbesserung seiner Cesundheit. Cehen ist eine unkomplizierte Form der moderaten Bewegung, die sehr einfach in den Alltag integriert werden kann. Das Fitnessprogramm orientiert sich dabei an den Empfehlungen nationaler und internationaler Organisationen, die zum Erhalt und zur Verbesserung der physischen Gesundheit ein »Tagespensum von 10.000 Schritten« definieren. «Quelle: https://www.tk.de/techniker/unterneh mensseiten/unternehmen/die-tk-app/tk-app-fitnessprogramm-2023654 Auch die IKK Cesund Plus schreibt: »Bei den zurückgelegten Schritten stellt sich jedoch die Frage: Wie viele sollen es sein? Man geht davon aus, dass rund 10.000 am Tag bereits mehr Fitness bringen, zum Erhalt der Cesundheit beitragen und Übergewicht entgegenwirken.«Quelle: https:// www.ikk-gesundplus.de/gesundheit/sport_bewegung/schrittzaehler/ (zuletzt aufgerufen am 10.06.2018).

69 Apple CEO Tim Cook während der Präsentation der Apple Watch. Quelle: https://www.the guardian-.com/technology/2015/feb/11/tim-cook-apple-watch-health-benefits (zuletzt aufgerufen am 05.06.2016). Apple expandiert in den Markt der Cesundheitsdaten. Über die App Health Records sollen Benutzer*innen Cesundheitsdaten und Krankenakten zentralisieren und teilen können: »Empower your patients with Health Records on iPhone.« Quelle: http s://www.apple.com/healthcare/health-records/ (zuletzt aufgerufen am 10.06.2018). Amazon plant derweil zusammen mit Berkshire Hathaway und der Investmentbank JPMorgan Chase eine eigene Krankenkasse. Auch Coogle investiert Risikokapital in diesen Bereich. 
Obwohl leicht erkennbar ist, dass derartige Aussagen auf der für Werbeformeln typischen Dramatik basieren, werden sie dennoch im Kontext von medizinischen Expertenaussagen und den Statistiken der Gesundheitsbehörden rezipiert:

»Apple Watch comments may be seen as distasteful in some quarters, but the dangers of sedentary lifestyles are more than a marketing line. $\aleph^{70}$

Trotzdem die Radikalität der Aussage hier in Zweifel gezogen wird, trägt sie doch $\mathrm{zu}$ einer diskursiven Sedimentierung einer monokausalen Ursacheninterpretation von im Grunde vielen verschiedenen und sehr komplexen Krankheiten bei, durch die die Verantwortung erneut individualisiert und Handlungsoptionen noch enger an technologische Lösungen gekoppelt werden. Vorreiter etwaiger Bonusprogramme in Deutschland war das Programm Vitality des Versicherungsdienstleisters Generali, der Self-Tracking und Selbstauskunfts-Applikationen ${ }^{71}$ in Verbindung mit Berufsunfähigkeits- und Risikolebensversicherungen einführte. ${ }^{72}$ Andere Krankenkassen sind schnell gefolgt. ${ }^{73}$ Neben den großen Versicherungs-

70 Quelle: https://www.theguardian.com/technology/2015/feb/11/tim-cook-apple-sitting-the-ne w-cancer (zuletzt aufgerufen am 05.06.2016).

71 »Die Vitality-Mental-Tests (Online-Fragebögen) helfen dabei, die emotionale und psychische Gesundheit besser einzuschätzen und persönliche Stressfaktoren zu erkennen.«Quelle: Quelle: https://netzpolitik.org/2018/tracking-durch-die-versicherung-zu-risiken-und-nebenwi rkungen/ (zuletzt aufgerufen am 10.06.2018).

72 Quelle: https://www.generali.de/ueber-generali/presse-medien/pressemitteilungen/versi cherung-neu-denken---generali-vitality-geht-an-den-start-10844/ (zuletzt aufgerufen am 06.10.2018). Die Versicherten können dazu Hardware und Apps mit einem speziellen Portal synchronisieren um Aktivitätspunkte zu sammeln. Cenerali orientierte sich dabei an einem Telemonitoring-Programm der südafrikanischen Versicherung Discovery. »Wir beeinflussen das Verhalten unserer Kunden, [denn] gesündere Kunden sind besser für uns. « Mario Creco - Chef des Cenerali-Konzerns. Quelle: Quelle: www.faz.net/aktuell/feuilleton/generali-a pp-preisnachlass-bei-zusenden-der-koerperdaten-13287991.html (zuletzt aufgerufen am 11.12.2014). »The healthier your lifestyle the bigger the rewards!« IHS/Discovery. Quelle: www.lifestyle-benefits.co.za/get-discovery-vitality/ (zuletzt aufgerufen am 11.12.2014). Die Liste an Ländern in denen das Vitality- Programm von Discovery angeboten wird verlängert sich dabei unentwegt: »Discovery is also offered in the UK, branded as »VitalityHealth« and »VitalityLife«. In addition, Vitality has built partnerships with insurance companies all over the world to introduce similar programs, for example with AIAin Asia and Ping An Health in China« (Christl und Spiekermann 2016: 67).

73 »Die DAK bietet ihren Klient*innen die Smartphone-App FitCheck an. Wer Versichertennummer, Cewicht und Sportaktivitäten angibt, kann über ein Bonusprogramm bei den Beiträgen sparen. Und Versicherte der Daimler-Betriebskrankenkasse erhalten ebenfalls eine Geldprämie, wenn sie ihre sportliche Leistung mit der App Runtastic belegen.«Auch die AOK Nordost bietet ihren Klient*innen in Zusammenarbeit mit dem Schweizer Fitness-Portal Dacadoo das Pilotprojekt AOK mobil vita an. Quelle: www.rp-online.de/digitales/neugierige-krankenkas sen-nutzen-apps-zur-datensammlung-aid-1.4211696 (zuletzt aufgerufen am 14.03.2015). Die Techniker Krankenkasse hat inzwischen ebenfalls ein entsprechendes Bonusprogramm ge- 
firmen United Health, Humana, Cigna and Highmark, die das Tragen von Wearables vor allem durch Sachgüter entlohnen, bietet etwa die John-HancockVersicherungsgesellschaft ${ }^{74}$ einen Beitragserlass an insofern die Klinet*innen die Daten ihrer Fitness-Armbänder mit den Servern von Apple's HealthKit platform synchronisieren (Christl und Spiekermann 2016: 66), das Portfolio derartiger Angebote wird in den USA zudem von einer inzwischen kaum mehr überschaubaren Anzahl an Startups ${ }^{75}$ ergänzt, die an der Individualisierung von Versicherungsleistungen arbeiten. In Italien ist das Anbieten eines Bonus-Tarifs inzwischen gesetzlich vorgeschrieben. ${ }^{76}$

Parallel formieren sich auch Anbieter wie Wellmo (»Simple \& easy selftracking «), ${ }^{77}$ die sich darauf spezialisiert haben »Gesundheitsdienstleister und Versicherungsgesellschaften « technisch und beratend dabei zu unterstützen »effektive, motivierende und skalierbare Gesundheitsprogramme ${ }^{78}$ durchzuführen, um Kosten $\mathrm{zu}$ reduzieren und den Gesundheitszustand der Klient*innen $\mathrm{zu}$ verbessern:

»Wearables, health and wellbeing apps and integrated health sensors are revolutionizing the way we keep track of our health and wellbeing. Increasingly many corporate wellbeing providers, preventative health care providers and health insurers are exploring self-monitoring tools and digital channels to develop new ways to support their clients. $\ll^{79}$

startet: »Das Fitnessprogramm ist ein neues Angebot des TK-CesundheitsCoachings, das Sie mit der TK-App nutzen können. Es unterstützt Sie zwölf Wochen lang dabei, mehr Bewegung in Ihren Alltag zu bringen. Dokumentieren Sie über Ihre Schritte Ihre Aktivität und lassen Sie sich von den begleitenden Fitnesslektionen in Schwung bringen.«Zusätzlich bietet die TK Apps für die Erfassung von Migräne-Symptomen und die Durchführung von Entspannungsübungen an. Quelle: https://www.tk.de/techniker/unternehmensseiten/unternehmen/die-tkapp/tk-app-fitnessprogramm-2023654 (zuletzt aufgerufen am 10.06.2018). Manulife bietet ein ähnliches Programm in Kanada an.

75 Ein einfaches Beispiel stellt das Unternehmen Oscar dar, das zurückgelegte Schritte mit Geschenkgutscheinen für den Amazon Versandhandel honoriert: »Cet the whole family active and earn Amazon ${ }^{\circledast}$ Gift Card rewards [...] « Quelle: https://www.hioscar.com/individuals/ny. Der US-Anbieter Beam bietet eine Zahnzusatzversicherung im Zusammenhang mit einer datensammelnden Zahnbürste, anhand derer Art und Regelmäßigkeit der Zahnpflege bemessen werden soll. Quelle: https://beam.dental/tech (zuletzt aufgerufen am 10.06.2018).

76 Quelle: https://ehealthblog.de/2015/03/26/generali-bringt-die-personalisierte-versicherung-n ach-europa/(zuletzt auf-gerufen am 10.06.2018).

77 Quelle: www.wellmo.com/ (zuletzt aufgerufen am 05.06. 2016).

78 Quelle: www.corporate-health-convention.de/de/messe/ausstellerverzeichnis/ (zuletzt aufgerufen am 05.06. 2016).

79 Quelle: www.wellmo.com/ (zuletzt aufgerufen am 05.06. 2016). 
»Die Wellmo Plattform verbindet die führenden Gesundheits-Apps, FitnessTracker und Wearables. ${ }^{80}$

Auch hier sind Anspielungen auf den euphorischen und kreativen Spirit der Technologieinnovationskultur häufig anzutreffen und werden durch die Entwicklungsgeschichten in einem angegliederten Blog gerahmt. ${ }^{81}$

»We are a team of experienced health tech enthusiasts, who want to help you create wellness programs that rock! $\ll^{82}$

Die Bedeutung, die vergleichsweise einfache Technologien wie Schrittzähler und die durch sie anfallenden Daten in diesem Zusammenhang spielen, erschließt sich dabei erst auf den zweiten Blick. Denn obgleich Bonusprogramme dieser Art auch ausschließlich selektiv einzelne Sportpraktiken fördern und ihre Durchführung überwachen könnten, zielen sie im buchstäblichen Sinne darauf ab sich schrittweise in alltägliche Tagesabläufe $\mathrm{zu}$ integrieren und hier ungenutzte Potentiale an die allgegenwärtige Logik finanzieller Verwertung anzuschließen. Im Spiegel numerischer Vergleiche werden Tätigkeiten, denen bisher kaum Aufmerksamkeit zugekommen ist, so zu einer potentiellen Ressource im Gesundheitswettbewerb:

»Bei Cenerali Vitality zählt wirklich jeder Schritt und bringt wertvolle Vitality Punkte. $\ll^{83}$

"You're already walking. Make your steps count. ${ }^{84}$

Die auf der aktiven oder passiven Verdatung beruhenden Versicherungstarife mögen derzeit noch nicht allzu weit verbreitet sein und vor allem in Form von auf Freiwilligkeit beruhenden Pilotprojekten in Erscheinung treten, dennoch weist vieles darauf hin, dass derartige Programme und Tarife mit zunehmender Verbreitung durch die voranschreitende Etablierung von Selbstvermessungsverfahren im Gesundheitswesen ihre Bonus-Logik zukünftig umkehren. Selbstvermessungspraktiken im Zusammenhang mit Versicherungsleistungen können über ihre Kopplung an flexible Tarife freiwillig bleiben und gleichzeitig bei Nichtnutzung zu monetären Nachteilen führen oder auf andere Weise schleichenden Druck auf abweichende Lebensstile ausüben - insbesondere dann wenn sie nur einen Teil eines Portfolios

80 Quelle: www.corporate-health-convention.de/de/messe/ausstellerverzeichnis/ (zuletzt aufgerufen am 05.06. 2016).

81 Quelle: www.wellmo.com/welcome-to-wellmos-blog/ (zuletzt aufgerufen am 05.06. 2016).

82 Quelle: www.wellmo.com/ (zuletzt aufgerufen am 05.06. 2016).

83 Quelle: https://www.generali-vitalityerleben.de/ (zuletzt aufgerufen am 10.06.2018).

84 Quelle: https://www.unitedhealthcaremotion.com/ (zuletzt aufgerufen am 10.06.2018). 
ausmachen, in das zusätzlich GPS-Daten, Daten aus Online-Aktivitäten und aus bargeldlosen Bezahlverfahren oder Bonus- und Payback-Karten eingehen.

Beispielsweise erhalten Teilnehmer*innen der HealthyFood-Option des Vitality-Programms von Humana Insurance, im Kontext dessen Angestellte ebenfalls dazu angehalten werden Wearables und Fitness-Devices zu tragen, ${ }^{85} \mathrm{Ra}$ batte auf als gesund bewertete Nahrungsmittel aus dem Sortiment von Walmart, dessen Angestellte sich ebenfalls bereits durch Technologien des Startups Zazengo tracken ließen.

»WalMart is currently one of Zazengo's clients. WalMart started using the platform for sustainability campaigns, and now is beginning to use it to orchestrate health and wellness campaigns. [...] By creating detailed feedback that tracks employee and departmental contributions at any level of the corporate hierarchy, Zazengo can be used to stage competitions and orchestrate rewards. ${ }^{86}$

Nicht zuletzt aus Sicht der Big-Data-Apologet*innen lassen sich all diesen Daten, allein dadurch dass sie untereinander in Beziehung gesetzt werden, zur Erzeugung neuer Informationsquellen heranziehen und führen zu Aussagen, Scores und Rankings, die für Individuen selbst kaum mehr zu ermessen sind. Es ist anzunehmen dass diese Zusammenführung von Daten, so wie sie aus anderen Bereichen, etwa der Customer-Analysen im Web bereits bekannt sind, durch die Weitergabe und den Verkauf von Daten durch Data-Broker oder innerhlab von Firmennetzwerken bereits Realität ist: Wie Wolfie Christl und Sarah Spiekermann schreiben, scheint es so, dass allein Fitbit Daten an mehr als 10 »third-party companies« weitergibt, $\mathrm{zu}$ denen auch das weltgrößte Datenhandelsunternehmen Acxiom zählt (Christl und Spiekermann 2016: 64). ${ }^{87}$

85 »Each employee will have a program designed just for them and their improved health, and they'll earn Vitality Points for activities such as wearing a fitness device, completing their Health Assessment and getting a flu shot. Your employees can earn Vitality Points for reaching certain target levels while wearing a fitness device, like a pedometer and for doing verified activities that could include biking to work, and taking a walk after dinner.« Quelle: https://www.humana.com/employer/humanavitality/ (zuletzt aufgerufen am 14.03.2015).

86 Quelle: www.indeed.com/cmp/Zazengo?from=reviews (zuletzt aufgerufen am 12.01.2016).

87 Und das sind nur die Handelskooperationen die auch tatsächlich in den Selbstauskünften enthalten sind. An wie viele Unternehmen die Daten tatsächlich weitergeleitet werden ist schwer abzuschätzen und wird nicht extern kontrolliert: »A study from 2013 analyzed 43 popular Android and iOShealth and fitness apps. They found that $39 \%$ of free apps and $30 \%$ of paid apps sent data to third parties not mentioned in the app or in any privacy policy (Christl und Spiekermann 2016: 64). Auf diesen verschlugenen Wegen (vgl. Christl und Spiekermann 2016: 63) gelangen die Daten der Activity-Tracker so möglicher Weise auch unabhängig von personalisierten Bonus-Programmen zu Versicherungsanbietern oder Arbeitgebern (vgl. Christl und Spiekermann 2016: 64). 
Ein ebenfalls sehr wesentlicher und weithin unbeachteter Aspekt dieser Entwicklung besteht darin, dass mit zunehmender Bedeutung und Verbindlichkeit individueller Messwerte auch die Kontrolle über die Erzeugungsbedingungen und ihre Validität erfolgen muss, die nur durch einen Ausbau entsprechender Überwachungssensorik erfolgen kann. Bereits jetzt enthalten die zugrundeliegenden Verträge entsprechende Klauseln denen nach z.B. die Weitergabe entsprechender Hardware an Dritte untersagt wird. Die Kontrolle darüber, dass ein Fitnessarmband nicht einfach dem Hund umgebunden wird oder sich sogar ein entsprechender Grauzonenmarkt im Gig-Work-Segment entwickelt, der auf dem Spazierentragen fremder Fitness-Tracker basiert, beginnt sich aber bereits heute über das Abfragen zusätzlicher Daten wie GPS oder die Erfassung der Puls-Signatur einer Person am Handgelenk $^{88} \mathrm{zu}$ realisieren. Hierdurch lassen sich wiederum zusätzliche Daten für die Lebensbedingungen und den Gesundheitszustand der Klient*innen und ihres unmittelbaren Umfeldes erheben.

Die freiwillige Abgabe selektiver Daten erzeugt somit zwangsläufig den Bedarf an weiteren Daten. Mehr noch als im Zusammenhang mit den WellnessProgrammen des betrieblichen Gesundheitsmanagements kann so zukünftig jeder Alltagsaspekt in den Inklusionssog datengetragener Versicherungsmodelle geraten - wodurch sich auch der kategorische Komparativ (vgl. Bröckling 2007: 282) der vergleichenden Selbstvermessung potentiell immer weiter mit unterschiedlichen Lebensbereichen verzweigt. Und das meint in erster Linie die Forcierung von gesundheitsökonomischen Konkurrenzbeziehungen innerhalb von Kohorte, Risikogruppen, Unternehmensbelegschaften oder Familien.

Diese Bonusprogramme stellen damit bereits heute in Aussicht, die bisherige Logik von Versicherungsleistungen in Zukunft mit einer ständigen Beweispflicht der Versicherten zu verweben, die aktiv bedient werden will. ${ }^{89}$ Spätestens an dieser Stelle greifen zwei Stränge des Self-Tracking-Diskurses rekursiv ineinander und bewirken das Gegenteil dessen, wofür die Self-Tracker*innen offiziell eintreten. Denn die Versorgung, die einem Individuum durch das Gesundheitswesen zu Teil

88 Quelle: https://netzpolitik.org/2018/tracking-durch-die-versicherung-zu-risiken-und-nebenwi rkungen/ (zuletzt aufgerufen am 10.06.2018).

89 Zudem beginnen auch Negativ-Konditionierungen (ähnlich wie im Fall des ProduktivitätsTools Beeminder) schleichend zu einer akzeptierten Praktik zu werden: »Other companies are experimenting with punishment schemes instead of rewards. The U.S. startup StickK offers an app that incorporates data from wearables, but instead of collecting »wellness points«, points are deducted if users do not achieve their activity goals. StickK'soffer to consumers is based on a kind of »contract«, in which users commit to donate a certain amount of money to specific charities when they are not achieving their goals. StickKargues that their approach is »far more effective than offering rewards « and already had 13 corporate customers in 2014, including three Fortune companies« (Christl und Spiekermann 2016: 68). 
wird, gerät in noch stärkere Abhängigkeit von massenhaften, kompetetiven Vergleichsmaßen, deren Datengrundlage nun durch die individuellen Selbstvermessungsanstrengungen ergänzt wird.

"BP's corporate wellness program is managed by Fitbit's partner StayWell, according to Forbes, a "population-management firm « who manages the collected health data as a »neutral third party«. StayWell describes itself as a »health engagement company«, whose "population-specific programs« are »backed by decades of experience and deep expertise in the science of behavior change « (Christl und Spiekermann 2016: 66).

Die dahinterstehende Methodizität verfeinert im Grunde nur den Zugriff biopolitischer Institutionen auf das Subjekt und die Vorstellung, die es von sich selbst unterhält. D.h. die institutionelle Ausweitung von Kontroll- und Überwachungspraxen stellt zwar den rechtlich-materiellen Rahmen von Subjektivationsprogrammen dar, ihre gesellschaftliche Bedeutung besteht darüber hinaus aber vor allem in der Erhebung von ökonomischen Versicherungslogiken zu sozialen Organisationsprinzipien schlecht hin: What is important here is that we are seeing the proliferation of consulting - a way of thinking, ordering, calculating, and acting on the world - rather than primarily the actions of consultants as identifiable agents" (Amoore 2013: 19).

Dabei sind es nun gerade die individualisierten Datenquellen, die - zu einem Datensatz zusammengeführt - vor allem eine Benachteiligung chronisch kranker Menschen oder Menschen mit Behinderung nach sich ziehen können. Über tarifliche und ideelle Förderung von (versicherungs-)wirtschaftlich Handelnden bzw. der damit zusammenhängenden Akteursfiktion des unabhängigen, rationalen und optimierenden Quantrepreneurs seiner selbst, konturieren sich diskursiv zudem auch immer stärker die Facetten seines Gegenübers - des unzulänglichen Individuums (vgl. Bröckling 2007: 289). 

IV. Schluss 



\title{
1 Progressive Selbstverdatung oder die Pflicht zum Wissen
}

\author{
»Ich bin sehr stolz darauf, dass manche \\ Leute glauben, ich sei eine Gefahr für die \\ geistige »Cesundheit«der Studenten.« \\ Foucault (2005c[1980-1988]: 963)
}

Selbstverdatungsprojekte im Zusammenhang mit der Erforschung, Behandlung und Kompensation chronischer Erkrankungen und Behinderungen gehören zu den Beispielen, an denen sich am ehesten emanzipative Ambitionen identifizieren lassen, die nicht gänzlich in den sozialpädagogischen Leitformeln des Empowerment aufgehen und reduktionistische Rationalisierungsthesen, die schon die Motive der Self-Tracker*innen auf einen ökonomistisch überformten Optimierungsantrieb festlegen wollen, ein Stück weit ausbremsen. Folgt man den Selbstbeschreibungen chronisch kranker Self-Tracker*innen, führen ihre Verdatungspraktiken zu einer Verbesserung ihrer Lebensqualität und bieten Möglichkeiten auf verschiedenen Ebenen repressiven Tendenzen entgegenzuwirken, die sie z.B. durch medizinischen Paternalismus oder soziale Entmündigung auf den Status benachteiligter Minderheiten festschreiben. Sie werden zudem als alternative Forschungsmethoden für Krankheiten diskutiert, die dem wissenschaftlich-medizinischen Betrieb auf Grund ihrer Seltenheit als zu unwirtschaftlich gelten. Die zwar geringe, diskursiv aber sehr präsente Anzahl an Self-Tracker*innen mit chronischen Erkrankungen oder körperlichen Behinderungen machen den Zusammenhang zwischen Selbstverdatung und progressiver Opportunität gegenüber den gesundheitspolitischen oder medizinisch-pharmazeutischen Autoritäten (vgl. Foucault 1973; 2002b[1971]: 829f.) und der Datengrundlage auf der sie operieren, damit sehr deutlich (vgl. Bowker und Star 2000).

»[We] need alternate methods for doing research that does not rely on large sample sizes. $\ll^{1}$ 
Die Ingenieurin und Co-Organisatorin des Quantified-Self-Meetups in Stockholm, Sara Riggare, legt z.B. in einer ganzen Reihe von Präsentationen und Blogartikeln dar, wie die anfänglich experimentelle und später routinierte Selbstverdatung ihrer Parkinsonkrankheit zu einer Erhöhung ihrer Lebensqualität und einer Verminderung ihrer Abhängigkeit von der Massenmedizin geführt hat:

"Self-tracking is, in my opinion, the most powerful weapon I can wish for in my battle against Parkinson's disease (PD) and I will explain why. I see my neurologist once or twice a year, about half-an-hour every time. That is one hour per year, and the rest of the year's 8765 hours, I spend in selfcare. [...] And this is where selftracking comes in. ${ }^{2}$

»I manage a complex regime of medication for Parkinson's disease. Self tracking allows me to re-calibrate my dosages after adding a new medication to the mix. $\aleph^{3}$

Unabhängig von einer verbesserten und lediglich gefühlsbasierten Selbstmedikamentierung, bzw. der Abschätzung von Kreuzreaktionen mit Ernährungs- oder Verhaltensweisen, ermöglicht die systematische Erhebung von Daten eine eigene

ding No. 83.«Quelle: http://quantifiedself.com/2017/03/reading-83/ (beide zuletzt aufgerufen am 12.12.2017).

Quelle: www.riggare.se/2014/07/30/self-tracking-part-1/ (zuletzt aufgerufen am 21.12.2017). Quelle: http://quantifiedself com/2017/05/qs17-preview-managing-parkinsons-8765-hours$y$-ear/ (zuletzt aufgerufen am 12.12.2017). Self-Tracking-Daten werden im medizinischen Betrieb in der Regel als sog. »unstrukturierter Daten« bezeichnet, denen in der Regel auf Crund unklarer und schwer zu überprüfenden Erhebungsmethoden die Validität abgesprochen wird. Obgleich es auf Seiten der Hersteller medizinischer Geräte inzwischen diverse Verzweigungen mit der Quantified-Self-Community gibt, bleiben nennenswerte Durchbrüche in diesem Bereich bisher aus. Die experimentelle und weniger wirtschaftlich ambitionierten Segmente der Quantified-Self-Community thematisieren diesen Umstand allerdings meist in Verbindung mit der Annahme eine strukturellen Weigerung medizinischer Professionen und kommerzieller medizinischer Dienstleister selbsterhobene Daten in die Diagnostik und Therapie mit einzubeziehen. In dem Quantified-Self-Newsletter »What we are reading No. 38« vom 29.11.2014 verlinkt Sara Riggare zu diesem Thema eine Studie, die durch eine Community von Diabetiker"innen initiiert wurde: »What happens when patients know more than their doctors? Experiences of health interactions after diabetes patient education: a qualitative patient-led study« Quelle: http://bmjopen.bmj.com/conten t/3/11/e003583. full (zuletzt aufgerufen am 12.12.2017). Im Newsletter heißt es dazu: »Some interesting findings about how healthcare providers may be uncomfortable with patient who understand themselves and their condition."Quelle: http://quantifiedself.com/2014/11 /reading-38/(zuletzt aufgerufen am 12.12.2017). Ein weiteres Beispiel stellt der Artikel: dar. [Nur noch über Wayback-Maschinen verfügbar] Quelle: http://web.archive.org/web/2017021 1081849/https://www.nytimes.com/2017/02/04/business/his-doctors-were-stumped-then-hetook-over.html?mtrref=quantifiedself.com\&gwh=BB0203AF649F94BB3735D3D63A484017\&g wt=pay (zuletzt aufgerufen am 12.12.2017). 
Artikulation bzw. Selbstherstellung im Diskurs anhand der hier vorherrschenden numerischen Äußerungsmodalitäten - also in der Sprache der medizinischen Wissenschaft. Sie lassen sich daher bedingt in der Tradition von anderen "uncountables« (vgl. Bowker 2000; Bowker und Star 2001; Röhle 2017) sehen und gehen über die Kritik daran in entsprechenden Datensätzen keinen Platz zu haben (vgl. Waring 1999[1988]) ${ }^{4}$ durch die Erstellung eigener Datensätze hinaus.

»Not enough data exists to explain the links between osteoporosis and metabolism on an individual basis, making data like Justin's important to our awareness of the cross-system nature of the disease. $\ll^{5}$

Das nach Gesundheit und Kontrolle strebende Subjekt, das an einer Datenbasis arbeitet, die den Spezifika des individuellen Lebens mehr Rechnung trägt, lässt sich mit all seinen Unbequemlichkeiten für den medizinischen Normalbetrieb daher sicherlich nicht auf den generellen Nutzen reduzieren, den es für den modernen Staat und die postfordistische Ökonomie hat (vgl. Rose 1999). ${ }^{6}$

Dennoch sind die Bestrebungen nach Autonomie selbst nicht autonom, sondern nur als relationales Verhältnis zu begreifen, das es mit gesellschaftlichen Normen und materiellen Zielbestimmungen unterhält, die bestimmte Vorstellungen von Freiheit erwecken und einen bestimmten Gebrauch erlangter Freiheiten nahelegen (Lemke 2010: 253). So wird die Art der Selbstherstellung chronisch kranker Menschen im Diskurs des Self-Tracking merklich durch verschiedene Frames angeleitet, beeinflusst und eingedämmt. Träger*innen von chronischen Erkrankungen oder Menschen mit Behinderungen erscheinen unter den Quantified-SelfMitgliedern daher einerseits kongruent zu einem »Diskurs der Differenz, der das Leben in Begriffen von »Abwesenheiten «, »Fehlern« und »Defekten « analysiert «, als defizitär (Lemke 2010: 237[Hervorhebungen im Original]); andererseits allerdings auch als aktive, tatkräftige, progressive und optimistische Gestalter*innen ihrer eigenen Lebensbedingungen. In beiden Fällen bilden dabei aber jene Körper- und Leistungsbezogenen Normen der Selbstbestimmung die Fixpunkte, die im Zusam-

4 Siehe vor allem den zweiten Abschnitt (»A Calling to Account«) der Studie »Counting for Nothing- What men value and women are worth.«

5 Quelle: http://quantifiedself.com/2017/05/qs17-preview-taking-osteoporosis/ (zuletzt aufgerufen am 04.12.2017).

6 Coggin und Newell übertragen die enstprechenden Thesen die Rose zum Nutzen aktiver Bürger aufgestellt hat auf das Empowerment von Menschen mit Behinderungen: »Our argument is if disability shares the trend to »active citizenship « that is of a piece with the contemporary form of governmentality that Rose identifies, then a specific modality of governmentality exists that relates to the power relations of disability - a modality symbolized by the way that people with disability are overlooked as »active citizens, « lingering on the margins of the gouvernable« (Goggin und Newell 2005: 272). 
menhang mit sozialpolitischen Empowermentkonzepten und der für sie typischen Semantiken bereits beschrieben wurden.

»]ustin Lawler: »At the age of 38, I was diagnosed with osteoporosis. After exhausting the usual route of blood tests \& scans from the doctors, I took things into my own hands and uncovered deeper health issues underlying the initial diagnosis«. $\ll^{7}$

Im gleichen Maße, in dem für die operativen Modi des medizinischen Betriebs individuell wirkungsvollere Alternativen erarbeitet werden, erfüllen sich so die gouvernementalen Prämissen (d.h. die Herstellung von Eigenständigkeit und Funktionsfähigkeit) auf einem verschlungenen Sekundärweg. Zwar gehen Biopolitik und Selbstbestimmtheitsstreben hier zunächst Hand in Hand, allerdings erzeugt ihr Zweckbündnis gleichzeitig diskursive Exklusionsbereiche, die feste Grenzen des Sagbaren markieren und die verschiedene Handlungsoptionen oder Selbstbilder aus dem Diskurs ausschließen: Etwas einfach nicht zu können oder dauerhaft auf die Hilfe anderer angewiesen zu sein, diese Hilfe einzufordern oder anderen zukommen zu lassen, ist in einem strikt lösungsorientierten Unabhängigkeitsprojekt wie dem Self-Tracking-Diskurs keine tragfähige Aussage. Ferner wird ein kausaler Zusammenhang zwischen Selbstbestimmung, bestimmten Formen des Wissens über sich selbst und einem besseren Leben unterstellt, der das Recht darauf etwas nicht $\mathrm{zu}$ wissen kategorisch überformt - mit dem Ergebnis, dass man in dieser Art von informationeller Selbstbestimmung nicht nicht mündig sein kann, ohne dass diese Nichtmündigkeit als technisches Problem oder ein korrekturbedürftiges Rationalitätsdefizit erscheint (vgl. Lemke 2010: 238, 253). Der Unwille die eigenen Möglichkeiten bis auf das Maximum auszuschöpfen wird so zur eigentlichen Anomie im Diskurs.

Anders als es z.B. Lemke im Zusammenhang mit der zunehmenden molekularbiologischen Neubetrachtung von phänotypischen Krankheiten als schicksalhafte Defizite des Genotyps beschreibt, findet hier, trotz zunehmender Objektivierung und Rationalisierung der Art und Weise über Krankheiten zu sprechen, aber gerade keine Emanzipation der Krankheit von den Kranken statt. Vielmehr stellt die experimentelle Herstellung und Nutzung von Informations- und Medientechnologien eine weitaus engere Beziehung zwischen Individuen und ihren Krankheiten im Sinne eines Healthisms (Lupton 2014) her und etabliert eine Art Stellvertreterdiskurs, der die Ursachen und die Verantwortung für Krankheiten oder dauerhafte Beeinträchtigungen im Verhalten des Individuums situiert, anstatt sie auf der Ebene gesellschaftlicher Strukturbedingungen zu suchen: »[T]echnological solutions are

7 Ankündigung des Vortrags von Justin Lawler auf der Quantified Self Europe Conference. Quelle: http://quantifiedself.com/2017/05/qs17-preview-taking-osteoporosis/ (zuletzt aufgerufen am 04.12.2017). 
held out for their potential to abolish or ameliorate the disability that is seen to lie within the individual « (Goggin und Newell 2005: 263); mit dem Effekt, dass lediglich nach der eigenen Anpassung an die gesellschaftlichen Bedingungen gestrebt wird, anstatt die gesellschaftlichen Voraussetzungen an die vielfältigen Bedürfnisse der Individuen anzupassen. Und dies selbst dann, wenn sie als (Teil-)Ergebnis aus der Analyse von Krankheitsursachen hervorgehen:

»[Osteoporosis] is more prevalent in people who work desk jobs [...]. ${ }^{8}$

In den Tabellen und Korrelationsmatrizen erscheinen die identifizierten Defizite als Verbindungen zwischen Ist-Zustand und freiheitlichen Entscheidungen in Bezug auf die vergangene Lebensweise, womit die Self-Tracker*innen in Form introspektiver Diagnosezirkel immer wieder auf sich selbst zurückfallen.

»[...] self-tracking can help those with chronic conditions spot associations between symptoms and lifestyle that a clinician might not have time to uncover. These personal discoveries can help improve one's health. $\ll^{9}$

Semantisch drohen diese Argumentationen dabei immer vom Modus der vermeintlich nüchternen numerischen Ursachenanalyse des Auftretens, der Chronifizierung oder der temporären Verschlechterung einer Erkrankung in eine Schuldbewertung umzuschlagen, die (und das ist ein entscheidender Unterschied $\mathrm{zu}$ anderen, ähnlich verlaufenden gesellschaftlichen Entwicklungen) nicht primär normativ gesetzt ist, sondern eine diskursfähige Beweislast produziert, die für sich selbst zu sprechen scheint und die die Freiheit des Individuums als verspielte Chance erscheinen lässt.

») If I saw in real time what my lifestyle was doing to my health ten years ago, I would have changed then « $^{10}$

Selbstbestimmung heißt im Zusammenhang mit Self-Tracking immer auch Selbstproblematisierung und Befreiung meint immer auch die Befreiung von zweifelhaften Wünschen und Gewohnheiten (vgl. Lemke 2010: 253). Und dies nicht nur in Bezug auf vergangenes sondern auch gegenwärtiges Verhalten und seine zukünftigen Auswirkungen auf das eigene Leben und die eigene Unabhängigkeit. Die Logik der numerischen Selbstüberwachung lässt sich so vom biopolitischen Paradigma der Quelle: http://quantifiedself.com/2017/05/qs17-preview-taking-osteoporosis/ (zuletzt aufgerufen am 04.12.2017).

9 Quelle: http://quantifiedself.com/2017/07/qs17-amsterdam-highlight-taking-osteoporosis/ (zuletzt aufgerufen am 04.12.2017).

10 Ankündigung des Vortrags von Justin Lawler auf der Quantified Self Europe Conference. Quelle: http://quantifiedself.com/2017/05/qs17-preview-taking-osteoporosis/ (zuletzt aufgerufen am 04.12.2017). 
Prävention kaum abgrenzen, das einen gegenwärtigen Mangel an Selbstdisziplin als erstes Symptom einer potentiellen Krankheit in der Zukunft erscheinen lässt. Insbesondere in Bezug auf das weithin unbestimmte Feld der Gesundheit kann jede noch so unbedeutend wirkende Information spätestens in Korrelation mit anderen Daten zum fehlenden Puzzleteil eines Krankheitsbildes werden, weswegen die quantitative Methodik der Selbstvermessung prinzipiell nur auf eine unerreichbare Vollständigkeit ausgerichtet sein kann und in ihrem exponentiellen Vollzug die Freiheit des Nichtwissens mehr und mehr einschränkt.

»The self awareness that comes with self tracking can be useful across months and years, elucidating subtile patterns that might otherwise be undetectable. ${ }^{11}$

Die Befreiung von der erzwungenen Unmündigkeit im Paternalismus der Massenmedizin ist diskursiv daher eng mit der wachsenden Pflicht verbunden, zumindest den Status Quo individueller Gesundheitsinvestitionen zu erhalten und das eigene Engagement fortwährend unter Beweis zu stellen. Zwischen dem Tracking von Entwicklungsverläufen chronischer Erkrankungen und alltäglicheren Anomalien besteht daher formallogisch immer nur ein gradueller Unterschied. Vielmehr lassen sich durch die entsprechende Feinjustierung von numerischen Introspektionstechniken, korrekturbedürftige Mängel im Verhalten jeder Person finden, womit diese Techniken auf lange Sicht dazu neigen ihren eigenen Bedarf zu erzeugen und die soziale Gesundheitsnormen vor sich her zu treiben.

Die diskursiv erzeugte Verkopplung von Gesundheitszustand mit stereotypen Erfolgsnarrativen, die im Umkehrschluss Krankheit mit Exklusion und sozialem Abstieg assoziiert, verhilft einer individuellen Selbsthilfeanleitung wie Sara Riggares »How not to fall«" ${ }^{12}$ so $\mathrm{zu}$ einer Allgemeingültigkeit, die weit über chronische Erkrankungen hinausweist und vielmehr symptomatisch für den gesamten SelfTracking-Diskurs ist, in dem es immer auch darum geht, nicht zurückzufallen.

11 Quelle: http://quantifiedself.com/2017/07/qs17-amsterdam-highlight-taking-osteoporosis/ (zuletzt aufgerufen am 04.12.2017).

12 Ein Symptom von Sara Riggares Parkinson-Erkrankung bezeichnet sie plötzlich auftretendes "freezing of gait. «An-hand verschiedener Tracking-Verfahren entwickelte sie eine Methode um einer akuten Muskelversteifung zumindest soweit entgegenzuwirken, dass sich die Gefahr eines Sturzes und entsprechender Folgeverletzungen minimiert lies. Quelle: http://qua ntifiedself.com/2014/08/sara-riggare-fall/ (04.12.2017). 


\section{$2 N=1$ taugt nicht zum Prinzip ${ }^{1}$}

Da die individuelle Verdatung von chronischen Erkrankungen bzw. entsprechender Therapiemethoden als wirksame Methoden diskutiert werden, die Effektivität einer Medikalisierung zu erhöhen oder Nebenwirkungen gering zu halten, erscheinen sie diskursiv sicher als die bedeutendsten Errungenschaften unter den verschiedenen Self-Tracking-Technologien, insofern jedenfalls, als dass sie die Lebensqualität einzelner Individuen erhöhen und ihnen zu mehr Selbstbestimmtheit verhelfen können. Dennoch wird deutlich, dass Self-Tracking-Verfahren nicht automatisch zu Befreiungstechnologien werden, nur weil sie abseits von den Bereichen der Arbeitseffizienz und des Erhalts der Arbeitsfähigkeit betrachtet werden. Vielmehr wirken Individualisierung und Erhöhung von Verantwortung für das eigene Handeln als ubiquitäre Prämissen des Self-Tracking-Diskurses schlechthin. Das Subjekt auf dessen Produktion sich die Self-Tracking-Technologien ausrichten, wird durch sie weder im positiven Sinne noch im negativen Sinne direkt geformt, sondern vielmehr im Verbund mit dem Diskurs erzeugt, der auch das Self-Tracking als Technologie diskutiert und realisiert.

Die Antwort auf die weiterführende Frage, ob Self-Tracking-Technologien prinzipiell emanzipative Potentiale bergen, ist daher auch weit weniger auf der Ebene der technischen Instrumente und der durch sie verwendeten Symbolsysteme zu finden, sondern hängt stärker mit den Prämissen und der Organisationsform des sie hervorbringenden Diskurses zusammen.

Die letzten Überlegungen zur Herkunft und Beschaffenheit des quantifizierten Selbst betreffen daher noch einmal das mit dieser Form der Subjektivität einhergehende Autonomieverständnis - nun allerdings dezidierter mit Blick auf seine Positionierung zwischen Individualität und Gemeinschaft. Die dahinterstehende Überlegung ist, dass Self-Tracking-Technologien wichtige Funktionen für die Bildung progressiver Gemeinschaften übernehmen könnten, insofern ihnen nicht die Herstellung von Individualität als oberstes Prinzip zu Grunde liegen würde. Denn die Kompatibilität zwischen Selbstverwirklichungsansprüchen wird für die über- 
individuelle Kooperation umso mehr zum Problem, »je stärker die Originalität und Unvergleichlichkeit der Individuen in den Vordergrund rückt (Klinger 1995: 130).

So haben etwa die emanzipatorischen Kämpfe verschiedener sozialer Bewegungen der 1960er und 1970er Jahre deutlich gemacht, dass Heteronomie und Autonomie keine unvereinbaren Gegensätze darstellen. Ihr Engagement richtet sich auf die Erhöhung individueller Freiheiten ohne dabei in die Falle der Vereinzelung zu tappen. ${ }^{2}$

So gab etwa das feministische Boston Women's Health Book Collective verschiedene gemeinschaftlich erarbeitete Ratgeber heraus, die sich mit dem Ziel der Aufklärung und der Ermutigung an Frauen richteten. Als Strategie der Gegeninformation thematisierten sie vor allem den patriarchalen Bias in der medizinischen Versorgung, sowie der pharmazeutischen Forschung und die spezifischen Probleme, die mit deterministischen Funktionsrollen wie z.B. der Haushälterin und Mutter verbunden waren (bzw. es zu weiten Teilen bis heute sind). Auch hier spielt das unabhängige Erfassen von Daten eine große Rolle bei der eigenständigen Entwicklung von Verfahren zur unabhängigen Symptomanalyse von Krankheiten, die sowohl Linderung versprechen als auch die Kontrollmöglichkeit für die Diagnosen eines Medizinwesens verbessern sollten, das die Spezifika des weiblichen Körpers weithin ignorierte und einer patriarchalen Definition unterwarf (Blume und Preuschoft 1981: 408). Anders als im Self-Tracking-Diskurs erschöpfen sich diese Strategien aber nicht in der Errichtung individueller Arzt-Patient*innen-Verhältnisse, sondern zielen vielmehr darauf, individuelle Erfahrungen im medizinischen Betrieb auf gesellschaftlicher Ebene abzubilden. Sie machen bei der Verbesserung der eigenen Gesundheit nicht Halt, sondern zielen auf die Politisierung der Medizin als Archipel verschiedener Machtbeziehungen (Foucault 2005d[1980-1988]: 228; 2003c[1976-1979]: 76) und unternehmen den Versuch dieses Machtverhältnis durch kollektive Organisation zu verschieben (Blume und Preuschoft 1981: 411). ${ }^{3}$

Im Vorwort der Neuauflage von 1992 heißt es: »Doch ein Rückzug auf rein individuelle Möglichkeiten ist keine Lösung, denn es hat in den meisten Fällen zur Folge, dass die einzelne Frau sich überfordert: Sie fügt den traditionellen gesellschaftlichen Erwartungen selbst noch die neue Forderung Emanzipation hinzu.«

3 Ganz ähnliches gilt, wenn in dem feministischen Ratgeber »Unser Körper, unser Leben« danach gefragt wird: »Was kann ich am Arbeitsplatz tun um fit zu bleiben?« Auch wenn die lohnarbeitsförmige Organisation der Produktion als tragende Säule der westlichen Gesellschaften von der Kritik des Kollektivs unberührt bleibt, dient das hier diskutierte Problem allenfalls nachrangig dem Erhalt des Arbeitsvermögens. Die Frage zielt also nicht auf die Suche nach der besten Resilienzstrategie. Vielmehr geht es dem Kollektiv, schlicht um die Abwendung körperlicher Schäden, die das Individuum - in diesem Fall die Frau in der Rolle der Haushälterin oder der Bürokraft - durch monotone Tätigkeiten erleidet. Das Cesunde Selbst soll vielmehr (durchaus im Einklang mit dem fordistischen Arbeitsverständnis der damaligen Zeit) vor den Arbeitsbedingungen geschützt werden (Blume und Preuschoft 1980: 23). 
Diesem Kollektiv ging es damit gerade darum, die Ohnmacht des Individuums durch gezielte Vernetzung und Austausch zu überwinden und so auch zu einer Aufdeckung von Krankheitsursachen zu gelangen, die nicht deckungsgleich mit den Individuen und etwaigen Mängeln in der Selbstsorge sind:

»Wir brauchen eine Medizin, in der die Wechselwirkungen zwischen Krankheit (Gesundheit) und Gesellschaft mit einbezogen werden[...] « ${ }^{4}$

Auch in der Argumentation des Boston Women's Health Book Collective spielen Zahlen als vermeintliche Wahrheitsäquivalente eine tragende Rolle. Allerdings sollen die zitierten Statistiken dem Kollektiv dazu dienen gesellschaftliche Verhältnisse, strukturelle Ungleichheiten oder normative Entscheidungen im medizinischen Betrieb sichtbar zu machen. Die Selbstbehauptung erfolgt hier von der Position einer solidarischen Gemeinschaft aus. Die Verbesserung der eigenen Lebensgrundlagen wird hier als erster Schritt einer umfassenden Überwindung von struktureller Ungerechtigkeit gedacht. Über Durchschnittsangaben zu ungewollten Schwangerschaften oder einzelnen Krankheiten nehmen sie einzelne Individuen so ein Stück weit aus der Verantwortung für das eigene Unglück und zeigen z.B. über die Häufung bestimmter Probleme in einzelnen Bevölkerungsgruppen die gesellschaftlichen Ursachen auf, die sie gleichzeitig zum Fernziel ihrer politischen Interventionen machen (Blume und Preuschoft 1981: 384ff.).

Das Problem einer strukturellen Benachteiligung in gesellschaftlichen Alltagspraxen setzt sich allerdings nicht selten auf der Ebene der statistischen Abbildung gesellschaftlicher Zustände fort. Insofern liegt hier ein inhaltlicher Berührungspunkt mit dem Self-Tracking-Diskurs, in dem die Strukturblindheit staatlicher und wissenschaftlicher Datenerhebungsverfahren gegenüber spezifischen Missständen und individuellen Problemen geradezu ein identitätsgebendes Merkmal darstellt.

Zusammengenommen machen diese beiden Perspektivierungen nummerische Kollektive zumindest denkbar, die z.B. die Verbesserung des individuellen körperlichen und geistigen Wohls mit der Problematisierung gesellschaftlicher Krankheitsursachen parallel führen und die der Abschaffung von Missständen den Vorzug vor einer individuell-kompensatorischen Auseinandersetzung mit entsprechenden Problemlagen gibt.

Die Plattform >curetogether etwa ist mit einem Ansatz gestartet, der zumindest teilweise in die Richtung der Erforschung von Krankheiten und kollektiv fundierter Behandlungen weist: Anfänglich vernetzte die Plattform die auf Selbsteinschätzung basierenden Angaben der Teilnehmer*innen und differenzierte dabei die Kategorien >Symptome $<$, >Behandlung $<$ und >Ergebnis $<$. Die Daten wurden auch hier als Streuung um einen Mittelwert abgebildet. Im Ergebnis lieferten das Projekt 
so z.B. Daten über die Wirksamkeit von pharmazeutischen Produkten, die auf unmittelbarem Austausch der Anwender*innen basierten, förderte Umwelteinflüsse oder eben gesellschaftliche -Ursachen zu Tage, die durch die Evaluationsart und Darbietungsform aber als gemeinsames und damit auch politisierbares Wissen erschienen. Mit Blick auf den Self-Tracking-Diskurs stellt die Plattform eines der seltenen Beispiele dar, indem die spezielle Äußerungsmodalität der Zahlen semantisch mit dem Begriff »together « auftrat und sich nicht auf das Prinzip N=1 ausrichtete. Wer heute die Url des Projektes aufruft, erhält allerdings folgende Nachricht:

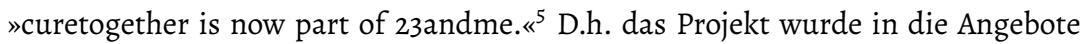
und damit auch die Datenpolitik eines Biotech-Unternehmens integriert an dem u.a. Google maßgeblich beteiligt ist. Ob der Idealismus der Seitenbetreiber*innen nun authentisch oder Teil einer für das Silicon Valley ${ }^{6}$ inzwischen obligatorischen Marketingsprache war, ändert zunächst nichts am Basisprinzip der Plattform, das prinzipiell in der Lage ist einzelne Werte von Individuen unter eigener Kontrolle in überindividuellen datenbasierten Vergleichen zu vernetzen. Womit diese seltene Form des Datensammelns sich dazu eignen würde die Massenbasis traditioneller Sozialpanels mit den Unabhängigkeitsbestrebungen der Self-Tracker*innen in Fragen der Erhebung, Verwaltung und Auswertung von Daten zu verbinden. Tatsächlich ist die Zahl derartiger Projekte allerdings sehr gering und die wenigen existierenden Plattformen sind dem kommerziell überformten Public-Health-Bereich zuzuordnen. Der auch den Ankerpunkt zur Quantified-Self-Community bildet. Der Self-Tracking-Diskurs weicht indes von seiner Fokussierung auf den radikalen Datenindividualismus kaum ab. Dabei sollen die kurzweiligen Erfolge vieler der dort diskutierten Praxen und Verfahren auch nicht in Abrede gestellt werden. Die Herstellung von äquivalenten Bedingungen, die Menschen mit strukturellen Benachteiligungen in die Lage versetzen am gesellschaftlichen Leben teilzunehmen, verhelfen Individuen partiell $\mathrm{zu}$ mehr Freiheit und mögen ihre Lebensbedingungen punktuell verbessern (teilweise auf enorme Weise). Aber als emanzipative Technologien müssten sie sich auch auf die Identifikation und Korrektur benachteiligender Strukturbedingungen selbst ausrichten. Der Self-Tracking-Diskurs bringt offenkundig keine numerischen Kollektive und Netzwerke hervor, die Individuen in die Lage versetzen Ursachen zu identifizieren, die außerhalb ihres Mikrokosmos liegen, sich die hierdurch entstehende Last unter einander zu teilen oder gemeinschaftlich gegen sie vorzugehen. Vielmehr zementiert er diese Zustände indem er kompensatorische Technologien hervorbringt, die ein längeres Aushalten

6 Das Startup Curetogether stammte aus Moutain View, Kalifornien. Auch die ähnlich funktionierende Plattform > Patientslikeme war von vornherein auf das Ziel ausgerichtet die durch Crowdsourcing generierten Daten an Pharmaunternehmen zu verkaufen. Quelle: https://ww w.patientslikeme.com/ (zuletzt aufgerufen am 27.08.2018). 
von krankmachenden Zuständen ermöglichen. Und dies gilt für chronisch kranke Self-Tracker*innen genau so wie für alle übrigen: Das quantifizierte Selbst zeigt sich als virtuos in der Entwicklung und Anwendung innovativer Resilienzstrategien, mit denen es auch bei hoher Belastung noch der eigenen Vernutzung trotzt. Aber sich dem zu widersetzen, was es auszuhalten gelernt hat und das außer Kraft zu setzen, was es so souverän bewältigt, kommt ihm nicht in den Sinn. ${ }^{7}$

»Statt einer Konklusion« formulierte Foucault in einem Aufsatz über den medizinisch-psychiatrischen Betrieb: »Es gibt in unserer Gesellschaft furchtbare Maschinen: Sie filtern Menschen, sortieren Geisteskranke, sammeln sie und sperren sie ein [...]«, man kann sie durch Rufe zu irritieren versuchen »[a]ber diese Rufe sind wirkungslos. Da sie von anderswoher und aus der Ferne kommen, sie sind nicht im Stande, die Maschine zu stören« (Foucault 2002c[1971]). Damit brachte er zum Ausdruck, dass die verschiedenen gesellschaftlichen Institutionen und Diskurse nach eigenen Regeln prozessieren und für die Interventionen anderer gesellschaftlicher Bereiche naturgemäß taub sind. Wohingegen das Boston Women's Health Book Collective die Stressreduktionsprogramme der Unternehmensgesundheit schon Ende der 1960er Jahre mehr oder minder ungehört als Vereinzelungsstrategien entlarvt und die mangelnde Thematisierung von Stressursachen bemängelt (Blume und Preuschoft 1980: 31), haben sich die Self-Tracker*innen indes auch über ihre Kreise hinaus Gehör verschaffen können und ihre Innovationen und Impulse werden über unterschiedliche Diskursgrenzen hinweg etwa im betrieblichen Gesundheitsmanagement aufgenommen. Allerdings ist dies mehr als ein Zeichen dafür zu deuten, dass sie sich bereits auf der konzeptuellen Ebene als anschlussfähig an die Operationsmodi moderner Arbeitsorganisation oder des Stressmanagements erweisen. Dort wo sie nicht ohnehin von vornherein wichtige Funktionen z.B. für die Aufrechterhaltung von Arbeitsvermögen und -leistung erfüllen, laufen sie immer Gefahr durch die Prämissen dieser angrenzenden Diskurse vereinnahmt zu werden. Denn sowohl gesundheitsbezogene Personalentwicklung als auch Krankenversicherungen schließen an zahlreiche Elemente des Self-Tracking-Diskurses an. Die Tatsache, dass ihnen eine solche Appropriation verhältnismäßig leicht gelingt mag damit zusammenhängen, dass zwischen den gesellschaftspolitischen Prinzipien der Self-Tracker*innen und denen der biopolitischen Agenturen weit weniger Differenzen bestehen, als weithin behauptet.

Das quantifizierte Selbst nimmt eine aktive Rolle in der Gesellschaftsgestaltung vielmehr dadurch ein, dass es die dezentrale Umgestaltung verschiedenster Lebensbereiche nach den Prämissen der modernen Ökonomie vorantreibt, die es $\mathrm{zu}$ einem essentiellen Bestandteil seines Selbstverständnisses gemacht hat. Das Unabhängigkeitsverständnis des quantifizierten Selbst zielt nicht auf die Unabhängigkeit von dezidierten Machtverhältnissen, vielmehr scheint es sich erst in 
einer Form von Eigenständigkeit zu erfüllen, die sich durch die völlige Kontrolle über alle es betreffende Belange herstellt. Entsprechend setzt es auch nicht bei der gemeinschaftlichen Kritik von Strukturproblemen an, sondern kämpft geradezu darum, die Ursachen für jedwede Form von Problemen in den eigenen Verantwortungsbereich zu ziehen.

Vor dem Hintergrund einer mit Vereinzelung gleichzusetzenden Individualisierung erzeugen Self-Tracking-Technologien dabei langfristig ihren eigenen Bedarf, da sie den Mangel an Fürsorge ausgleichen müssen, den sie selbst mitproduzieren: "Je abstrakter und distanzierter die objektive Ordnung der Dinge wird, desto intensiver wird die "Sorge um sich« (Klinger 1995: 226).

Auch für Foucault liegt die einzige Möglichkeit der Befreiung von einer derartigen Überformung durch eine unkontrollierte Kontrolle, die sich gerade über Freiheit und Individualität ins Werk setzt, daher in einer zunächst gegenläufig erscheinenden Beziehung zwischen Heteronomie und Autonomie. Denn obgleich Foucault kongruent zu den Formeln des gouvernementalen Empowerment konstatiert, »dass es keinen anderen, ersten und letzten Punkt des Widerstands gegen die politische Macht gibt als die Beziehung seiner selbst zu sich ${ }^{8}{ }^{8}$ kann dieser Prozess nur in Gemeinschaft und nicht in der gemeinschaftlichen Suche nach individuellen Problemlösungen erfolgen, die jeden Einzelnen und jede Einzelne notwendiger Weise auf sich selbst zurückwerfen. Wie Foucault im Aufsatz »Subjekt und Macht « schreibt, handelt es sich bei der Selbstermächtigung vielmehr gerade »um Kämp$\mathrm{fe}$, die den Status des Individuums in Frage stellen. Einerseits treten sie für das Recht auf Anderssein ein, und betonen alles, was die Individualität des Individuums ausmacht. Andererseits wenden sie sich gegen alles, was das Individuum zu isolieren und von den anderen abzuschneiden vermag, was die Gemeinschaft spaltet, was den einzelnen zwingt, sich in sich selbst zurückzuziehen, und was ihn an seine eigene Identität bindet. Diese Kämpfe werden nicht für oder gegen das »Individuum « ausgetragen, sondern gegen die "Lenkung durch Individualisierung«, wie man sie nennen könnte« (Foucault 2005b[1980-1988]: 274[Hervorhebungen im Original]). ${ }^{9}$

Damit besteht das Hauptziel emanzipatorischer Kämpfe der Gegenwart »zweifellos nicht darin, herauszufinden, sondern abzulehnen, was wir sind. Wir müssen uns vorstellen und konstruieren, was wir sein könnten, wenn wir uns dem doppelten politischen Zwang entziehen wollen, der in der gleichzeitigen Individualisierung und Totalisierung der modernen Machtstrukturen liegt« (Foucault 2005e[1980-1988]: 280).

Wenn die Machtstrukturen sich durch das Subjekt verwirklichen bzw. eins mit ihm sind, dann muss das Ablehnen dieser Strukturen zwangsläufig mit dem Ab-

8 Foucault (2004[1981-1982]: 313).

9 Zitiert nach Brieler (2007: 255f.). 
lehnen dessen einhergehen was man ist. Weder führen technologische Introspektionstechniken allein zu einer Unabhängigkeit, die sich von modernen Formen des Erhalts der ökonomischen Produktionsbedingungen und der Gesundheit des Bevölkerungskörpers merklich unterscheidet, noch reicht es, schlicht hinter die eigene Subjektivität zu sehen. Ein emanzipativer Technik-Diskurs setzt bei einem quantifizierten Wir an und muss sich fernab der Wünsche optimale Versionen des Selbst zu erzeugen der Frage widmen, woher diese Wünsche kommen. 



\section{Literaturverzeichnis}

Adorno, Theodor W.; Max Horkheimer (1969[1944]): Dialektik der Aufklärung. Frankfurt a.M.: Fischer.

Althusser, Louis (1977): Ideologie und ideologische Staatsapparate. Anmerkungen für eine Untersuchung, in: Ideologie und ideologische Staatsapparate. Aufsätze zur marxistischen Theorie. Hamburg: VSA, S. 108-153.

Aly, Götz; Roth, Karl Heinz (2005[1984]): Die restlose Erfassung. Volkszählen, Identifizieren, Aussondern im Nationalsozialismus. Frankfurt a.M.: Fischer.

Amoore, Louise (2013): The politics of possibility. Risk and security beyond probability. Durhum: Duke University Press.

Andersen, Niels Åkerstrøm (2003): Discursive analytical strategies. Understanding Foucault, Koselleck, Laclau, Luhmann. Bristol: Policy Press.

Angermüller, Johannes; van Dyk, Silke (2010): Diskursanalyse meets Gouvernementalitätsforschung. Perspektiven auf das Verhältnis von Subjekt, Sprache, Macht und Wissen. Frankfurt a.M. [u.a.]: Campus Verlag.

Bauer, Matthias; Ernst, Christoph (2010): Diagrammatik. Einführung in ein kulturund medienwissenschaftliches Forschungsfeld. Bielefeld: transcript.

Barbrook, Richard; Cameron, Andy (1995): The californian ideology - Mute Mix. Mute 1 (3). Online unter: www.hrc.wmin.ac.uk/theory-californianideology-mu te.html (zuletzt aufgerufen am 17.08.2018).

Barnes, Susan B. (2006): A privacy paradox: Social Networking in the United States, in: First Monday 11 (9). Online unter: http://firstmonday.org/article/view/1394/ 1312_2 (zuletzt aufgerufen am 17.08.2018).

Barth, Manuela (2009): »Wir nennen es Kreativität« - Inszenierungen von »alter« und »neuer « Arbeit in Werbebildern der Informations- und Kommunikationstechnologie, in: Irene Götz/Barbara Lemberger (Hg.): Prekär arbeiten, prekär leben. Kulturwissenschaftliche Überlegungen auf ein gesellschaftliches Phänomen. Frankfurt, New York: Campus, S. 183-204.

Becker, Guity Nashat (1998): Ökonomie des Alltags. Vom Baseball über Gleichstellung zur Einwanderung: Was unser Leben wirklich bestimmt, Tübingen: MohrSiebeck. 
Behrisch, Lars (2006): Vermessen, Zählen, Berechnen. Die politische Ordnung des Raums im 18. Jahrhundert. Frankfurt a.M. [u.a.]: Campus Verlag.

Bellinger, Andréa; Krieger, J. David (2014): Gesundheit 2.0 - Das ePatienten Handbuch. Bielefeld: transcript.

Betz, Fritz; Riegler, Johanna (2003): Bilder der Arbeit im Spätkapitalismus. Zum strategischen Machtverhältnis von Arbeit, Selbst und Technologien. Cultural Studies Band 5. Wien: Löcker.

Blume, Angelika; Preuschoft, Claudia (1980): Unser Körper - unser Leben. Ein Handbuch von Frauen für Frauen. Band I. Reinbek: Rowohlt.

Blume, Angelika; Preuschoft, Claudia (1981): Unser Körper - unser Leben. Ein Handbuch von Frauen für Frauen. Band II. Reinbek: Rowohlt.

Bodenschatz, Harald (1995): Analogismus von Fabrikarbeit und Alltag außerhalb der Fabrik: Ein Essential des Fordismus?, in: Regina Bittner (Hg.): Zukunft aus Amerika, Fordismus in der Zwischenkriegszeit, Siedlung, Stadt, Raum. Dessau: Stiftung Bauhaus Dessau, S. 39-43.

Boehm, Gottfried (2007): Unbestimmtheit - Zur Logik des Bildes, in: ders. (Hg.): Wie Bilder Sinn erzeugen - Die Macht des Zeigens. Berlin University Press, S. 199-213.

Bohn, Cornelia (2003): Mediatisierte Normalität: Normalität und Abweichung systemtheoretisch betrachtet, in: Jürgen Link/Thomas Loer/Hartmut Neuendorff (Hg.): »Normalität« im Diskursnetz soziologischer Begriffe. Heidelberg: Synchron, S. 39-50.

Boltanski, Luc; Chiapello, Eve (2003): Der neue Geist des Kapitalismus. Konstanz: UVK-Verlagsgesellschaft.

Bourdieu, Pierre (1998): Gegenfeuer. Konstanz: UVK-Verlagsgesellschaft.

Bowker, Geoffrey C.; Star, Susan L. (2000): Sorting Things out - Classification and its Consequences. Cambridge. MIT Press.

Bowker, Geoffrey C.; Star, Susan L. (2001): Pure, real and rational numbers: The American imaginary of countability, in: Social Studies of Science, 31 (3), S. 422425.

Bublitz, Hannelore; Bührmann, Andrea D.; Hanke, Christine; Seier, Andrea (1999): Diskursanalyse - (k)eine Methode? Eine Einleitung, in: Hannelore Bublitz/Andrea D. Bührmann/Christine Hanke/Andrea Seier (Hg.): Das Wuchern der Diskurse. Perspektiven der Diskursanalyse Foucaults. Frankfurt a.M. [u.a.]: Campus-Verlag, S. 10-21.

Bublitz, Hannelore (2003): Diskurs. Bielefeld: transcript.

Bublitz, Hannelore (2006): Differenz und Integration. Zur diskursanalytischen Rekonstruktion der Regelstrukturen sozialer Wirklichkeit, in: Reiner Keller (Hg.): Handbuch sozialwissenschaftliche Diskursanalyse. Theorien und Methoden. Wiesbaden: VS-Verlag, S. 227-262. 
Bublitz, Hannelore (2010): Im Beichtstuhl der Medien. Die Produktion des Selbst im öffentlichen Bekenntnis. Bielefeld: transcript.

Brieler, Ulrich (2007): Genealogie im »Empire«. Zum theoretischen Produktionsverhältnis von Antonio Negri und Michel Foucault, in: Clemens Kammler/Rolf Parr (Hg.): Foucault in den Kulturwissenschaften. Eine Bestandsaufnahme. Heidelberg: Synchron, S. 239-262.

Bröckling, Ulrich; Krasmann, Susanne; Lemke, Thomas (2000): Gouvernementalität, Neoliberalismus und Selbsttechnologien. Eine Einleitung, in: dies. (Hg.): Gouvernementalität der Gegenwart. Studien zur Ökonomisierung des Sozialen. Frankfurt a.M.: Suhrkamp, S. 7-40.

Bröckling, Ulrich (2003): You are not responsible for being down, but you are responsible for getting up. Über Empowerment, in: Leviathan 31 (3), S. 323-344.

Bröckling, Ulrich (2004): Menschenökonomie, Humankapital. Eine Kritik der biopolitischen Ökonomie, in: Ulrich Bröckling/Benjamin Bühler/Marcus Hahn/Matthias Schöning/Manfred Weinberg (Hg.): Disziplinen des Lebens. Zwischen Anthropologie, Literatur und Politik. Tübingen: Gunter Narr Verlag, S. 275-296.

Bröckling, Ulrich (2007): Das unternehmerische Selbst. Soziologie einer Subjektivierungsform. Frankfurt a.M.: Suhrkamp.

Bröckling, Ulrich (2010): Totale Mobilmachung. Menschenführung im Qualitätsund Selbstmanagment, in: Ulrich Bröckling, Susanne Krasmann und Thomas Lemke (Hg.): Gouvernementalität der Gegenwart. Studien zur Ökonomisierung des Sozialen. Frankfurt a.M.: Suhrkamp, S. 131-167.

Bröckling, Ulrich; Krasmann, Susanne (2010): Ni methode, ni approche. Zur Forschungsperspektive der Gouvernementalitätsstudien - Mit einem Seitenblick auf Konvergenzen und Divergenzen zur Diskursforschung, in: Johannes Angermüller/Silke van Dyk (Hg.): Diskursanalyse meets Gouvernementalitätsforschung. Perspektiven auf das Verhältnis von Subjekt, Sprache, Macht und Wissen. Frankfurt a.M. [u.a.]: Campus-Verlag, S. 23-43.

Bröckling, Ulrich (2012): Der ruf des Polizisten - Die Regierung des Selbst und ihre Widerstände, in: Reiner Keller/Werner Schneider/Willy Viehöver (Hg.): Diskurs - Macht - Subjekt: Theorie und Empirie von Subjektivierung in der Diskursforschung. Wiesbaden: VS-Verlag, S. 131-144.

Bröckling, Ulrich (2013): Der Mensch als Akku, die Welt als Hamsterrad. Konturen einer Zeitkrankheit, in: Sighard Neckel/Greta Wagner (Hg.): Leistung und Erschöpfung. Burnout in der Wettbewerbsgesellschaft. Berlin: Suhrkamp. S. 179201.

Bröckling, Ulrich (2013a): In der Optimierungsfalle. Zur Soziologie der Wettbewerbsgesellschaft, in: Supervision: Mensch, Arbeit, Organisation 31 (4), S. 4-11. 
Bröckling, Ulrich (2014): Jeder Mensch ein Künstler, jeder Mensch ein Unternehmer? Resonanzen zwischen künstlerischem und ökonomischem Feld, in: Zeitschrift der dramaturgischen Gesellschaft (1), S. 15-21.

Bröckling, Ulrich (2015): Gesteigerte Tauglichkeit, vertiefte Unterwerfung. Transformationen des Regierbarmachens, in: Soziopolis. Gesellschaft beobachten. Online unter: https://soziopolis.de/beobachten/kultur/artikel/gesteigerte-taug lichkeit-vertiefte-unterwerfung/ (zuletzt aufgerufen am 17.08.2018).

Bröckling, Ulrich (2017): Resilienz. Über einen Schlüsselbegriff des 21. Jahrhunderts, in: Soziopolis. Gesellschaft beobachten. Online unter: https://soziopolis .de/beobachten/kultur/artikel/resilienz/ (zuletzt aufgerufen am 17.08.2018).

Bröckling, Ulrich (2017b): Gute Hirten führen sanft. Über Menschenregierungskünste. Frankfurt a.M.: Suhrkamp.

Candeias, Mario (2008): Genealogie des Prekariats, in: Claudio Altenhain, Anja Danilina, Erik Hildebrandt, Stefan Kausch, Annekathrin Müller, Tobias Roscher (Hg.): Von »Neuer Unterschicht « und Prekariat: transcript, S. 121-138.

Christl, Wolfie; Spiekermann, Sarah (2016): Networks of control. A report on corporate surveillance, digital tracking, big data and privacy. Wien: Facultas-Verlag. Clough, Patricia Ticineto (2013): The Digital, Labor and Measure Beyond Biopolitics, in: Trebor Scholz (Hg.): The Internet as Playground and Factory. New York: Routledge, S. 112-126.

Cingolani, Patrick (2013): Wir die Prekarität ein neues Leitmodell der Arbeit?, in: Oliver Machart (Hg.): Facetten der Prekarisierungsgesellschaft. Prekäre Verhältnisse - sozialwissenschaftliche Perspektiven auf die Prekarisierung von Arbeit und Leben. Bielefeld: transcript, S. 23-36.

Cruikshank, Barbara (1993): Revolutions within: self-government and selfesteem, in: Economy and Society 22 (3), S. 327-344.

Cruikshank, Barbara (1999): The will to empower. Democratic citizens and other subjects. Ithaca, New York: Cornell University Press.

Czudaj, Antje (2016): Miranda July's Intermedial Art. The Creative Class between Self-Help and Individualism. Bielefeld: transcript.

Deflem, Mathieu (1997): Surveillance and Criminal Statistics: Historical Foundations of Governmentality. In: Studies in Law, Politics and Society, Volume 17. Greenwich, CT: JAI Press, S. 149-184.

Deleuze, Gilles (1993): Postskriptum über die Kontrollgesellschaft, in ders. (Hg.): Unterhandlungen 1972-1990. Frankfurt a.M.: Suhrkamp, S. 254-261.

Desrosières, Alain (1999): The history of statistics as a genre: styles of writing and social uses. Styles Of Writing And Social Uses. Auf der Konferenz »Statistical Internationalism, State Practices, and National Traditions: Progress, Report and Prospects in the History of Statistics«. Universität Quebec. Montreal, Canada, 22.09.1999. 
Desrosières, Alain (2001): How real are statistics?, in: Social Reasearch 68 (2), S. 339355.

Desrosières, Alain (2005): Die Politik der großen Zahlen. Eine Geschichte der statistischen Denkweise. Berlin: VS-Verlag.

Deutschmann, Christoph; Faust, Michael; Jauch, Peter; Notz, Petra (1995): Veränderungen der Rolle des Managers im Prozess der reflexiven Modernisierung, in: Zeitschrift für Soziologie, 24 (6), S. 436-450.

Diaz-Bone, Rainer (1999): Probleme und Strategien der Operationalisierung des Diskursmodells im Anschluss an Michel Foucault, in: Hannelore Bublitz/Andrea D. Bührmann/Christine Hanke/Andrea Seier (Hg.): Das Wuchern der Diskurse. Perspektiven der Diskursanalyse Foucaults. Frankfurt a.M. [u.a.]: Campus-Verlag, S. 119-135.

Diestelhorst, Lars (2016): Die Glühbirne und der Möbelpacker. Über den Begriff »Leistung« als leere Abstraktion, in: Felix Klopotek/Peter Scheiffele (Hg.): On time Run - Zonen der Selbstoptimierung. Berichte aus der Leistungsgesellschaft. Berlin: Matthes und Seitz, S. 35-52.

Durkheim, Emile; Herkommer, Hanne; Herkommer, Sebastian (2006[1897]): Der Selbstmord. Frankfurt a.M.: Suhrkamp.

Duttweiler, Stefanie (2007): Sein Glück machen. Arbeit am Glück als neoliberale Regierungstechnologie. Konstanz: UVK-Verlagsgesellschaft.

Duttweiler, Stefanie (2016): Alltägliche (Selbst)Optimierung in neoliberalen Gesellschaften, in: Politik und Zeitgeschichte, 66 (37-38), S. 27-32.

Duttweiler, Stefanie; Passoth, Jan-Hendrik (2016): Self-Tracking als Optimierungsprojekt?, in: Stefanie Duttweiler/Robert Gugutzer/Jan-Hendrik Passoth/Jörg Strübing (Hg.): Leben nach Zahlen. Self-Tracking als Optimierungsprojekt. Bielefeld: transcript, S. 9-42.

Duttweiler, Stefanie; Gugutzer, Robert; Passoth, Jan-Hendrik; Strübing, Jörg (2016b): Leben nach Zahlen. Self-Tracking als Optimierungsprojekt? Bielefeld: transcript.

Duttweiler, Stefanie (2017): Daten statt Worte?! Bedeutungsproduktion in digitalen Selbstvermessungspraktiken, in: Thorben Mämecke/Jan-Hendrik Passoth/Josef Wehner (Hg.): Bedeutende Daten. Modelle, Verfahren und Praxis der Vermessung und Verdatung im Netz. Wiesbaden: VS-Verlag, S. 251-276.

Dyk van, Silke; Angermüller, Johannes (2010): Diskursanalyse meets Gouvernementalitätsforschung - Zur Einführung, in: Johannes Angermüller (Hg.): Diskursanalyse meets Gouvernementalitätsforschung. Perspektiven auf das Verhältnis von Subjekt, Sprache, Macht und Wissen. Frankfurt a.M. [u.a.]: CampusVerlag, S. 7-20.

Ehrenberg, Alain (1991): Le Culte de la performance. Paris: Calmann-Lévy.

Ehrenberg, Alain (2011): Das erschöpfte Selbst. Depression und Gesellschaft in der Gegenwart. Frankfurt a.M.: Suhrkamp. 
Espeland, Wendy Nelson; Sauder, Michael (2007): Rankings and Reactivity How Public Measures Recreate Social Worlds, in: American Journal of Sociology 113 (1), S. 1-40.

Ewald, François (1993[1983]): Der Vorsorgestaat. Frankfurt a.M.: Suhrkamp. Fawcett, Tom (2015): Mining the Quantified Self: Personal Knowledge Discovery as a Challenge for Data Science, in: Big Data - 3 (4), S. 249-266.

Florida, Richard L. (2002): The rise of the creative class. And how it's transforming work, leisure, community and everyday life. Princeton, N.J: Recording for the Blind \& Dyslexic.

Florida, Richard L. (2003): Cities and the creative class, in Citiy and Community 2 (1), S. 3-19.

Foucault, Michel (1973): Die Geburt der Klinik. Eine Archäologie des ärztlichen Blicks. München: Hanser.

Florida, Richard (2012): The Rise of the Creative Class - Revisited: $10^{\text {th }}$ Anniversary Edition - Revised and Expanded. New York: Basic Books.

Foucault, Michel (1977): Überwachen und Strafen. Die Geburt des Gefängnisses. Frankfurt a.M.: Suhrkamp.

Foucault, Michel (1977b): Discipline and Punish: The Birth of the Prison, trans. A. Sheridan. New York: Vintage Books.

Foucault, Michel (1978[1974]): Von der Subversion des Wissens. Frankfurt a.M.: Fischer.

Foucault, Michel (1978): Wahrheit und Macht. Interview mit Michel Foucault von Allessandro Fontana und Pasquale Pasquino, in: ders. (Hg.): Dispositive der Macht. Über Sexualität, Wissen und Wahrheit, Berlin: Merve-Verlag, S. 21-54.

Foucault, Michel (1981[1969]): Archäologie des Wissens. Frankfurt a.M.: Suhrkamp. Foucault, Michel (1983[1976]): Der Wille zum Wissen. Sexualität und Wahrheit I. Frankfurt a.M.: Suhrkamp.

Foucault, Michel (1984): Der Gebrauch der Lüste. Sexualität und Wahrheit II. Frankfurt a.M.: Suhrkamp.

Foucault, Michel (1986): Die Sorge um sich. Sexualität und Wahrheit III. Frankfurt a.M.: Suhrkamp.

Foucault, Michel (1991[1966]): Die Ordnung der Dinge. Eine Archäologie der $\mathrm{Hu}-$ manwissenschaften. Frankfurt a.M.: Suhrkamp.

Foucault, Michel; Konersmann, Ralf (1991[1970]): Die Ordnung des Diskurses. Frankfurt a.M.: Fischer.

Foucault, Michel (1993[1988]): Die politische Technologie der Individuen, in: Luther H. Martin/Huck Gutman/Patrick H. Hutton (Hg.): Technologien des Selbst. Frankfurt a.M.: Fischer.

Foucault, Michel; Trombadori, Ducio; Brühmann, Horst (1996[1978]): Der Mensch ist ein Erfahrungstier. Gespräch mit Ducio Trombadori. Frankfurt a.M.: Suhrkamp. 
Foucault, Michel (2000): Die Gouvernementalität, in: Ulrich Bröckling/Susanne Krasmann/Thomas Lemke (Hg.): Gouvernementalität der Gegenwart. Frankfurt a.M.: Suhrkamp, S. 41-67.

Foucault, Michel; Ott, Michaela (2001[1975-1976]): In Verteidigung der Gesellschaft. Vorlesungen am Collège de France 1975/1976. Frankfurt a.M.: Suhrkamp.

Foucault, Michel (2001[1969]): »Wer sind Sie Professor Foucault?«, Gespräch mit P. Caruso, in: Daniel Defert/François Lagrange (Hg.): Schriften in vier Bänden. Dits et Ecrits, Band I: 1954-1969. Frankfurt a.M.: Suhrkamp, S. 770-793.

Foucault, Michel (2001a[1969]): Was ist ein Autor?, in: Daniel Defert/François Lagrange (Hg.): Schriften in vier Bänden. Dits et Ecrits, Band I: 1954-1969. Frankfurt a.M.: Suhrkamp, S. 1003-1041.

Foucault, Michel (2001[1975-1976]): In Verteidigung der Gesellschaft. Vorlesungen am Collège de France 1975/1976. Frankfurt a.M.: Suhrkamp.

Foucault, Michel (2002): Die Wahrheit und die juristischen Formen, Frankfurt a.M.: Suhrkamp.

Foucault, Michel (2002[1970-1975]): Nietzsche, die Genealogie, die Historie, in: Daniel Defert/François Lagrange (Hg.): Schriften in vier Bänden. Dits et Ecrits, Band II: 1970-1975. Frankfurt a.M.: Suhrkamp, S. 166-191.

Foucault, Michel (2002b[1970-1975]): Die psychiatrische Macht, in: Daniel Defert/François Lagrange (Hg.): Schriften in vier Bänden. Dits et Ecrits, Band II: 1970-1975. Frankfurt a.M.: Suhrkamp, S. 829-844.

Foucault, Michel (2002c[1970-1975]): Statt einer Konklusion, in: Daniel Defert/François Lagrange (Hg.): Schriften in vier Bänden. Dits et Ecrits, Band II: 1970-1975. Frankfurt a.M.: Suhrkamp, S. 519-522.

Foucault, Michel (2003[1973]): Die Wahrheit und die juristischen Formen. Frankfurt a.M.: Suhrkamp.

Foucault, Michel (2003[1976-1979]): Macht und Wissen, in: Daniel Defert/François Lagrange (Hg.): Schriften in vier Bänden. Dits et Ecrits, Band III: 1976-1979. Frankfurt a.M.: Suhrkamp, S. 515-534.

Foucault, Michel (2003b[1976-1979]): Die Zeit anders leben, in: Daniel Defert/François Ewald (Hg.): Schriften in vier Bänden. Dits et Ecrits, Band III: 1976-1979. Frankfurt a.M.: Suhrkamp, S. 984-987.

Foucault, Michel (2003c[1976-1979]): Krise der Medizin oder Krise der Antimedizin, in: Daniel Defert/François Lagrange (Hg.): Schriften in vier Bänden. Dits et Ecrits, Band III, 1976-1979. Frankfurt a.M.: Suhrkamp, S. 54-77.

Foucault, Michel (2004[1977-1978]): Sicherheit, Territorium, Bevölkerung. Geschichte der Gouvernementalität I: Vorlesungen am Collège de France 1978/1979. Frankfurt a.M.: Suhrkamp.

Foucault, Michel (2004[1978-1979]): Die Geburt der Biopolitik. Geschichte der Gouvernementalität II: Vorlesungen am Collège de France 1978/1979. Frankfurt a.M.: Suhrkamp. 
Foucault, Michel (2004[1981-1982]): Hermeneutik des Subjekts. Vorlesung am Collège de France 1981/1982. Frankfurt a.M.: Suhrkamp.

Foucault, Michel (2005[1979]): Omnes et singulatim: Zu einer Kritik der politischen Vernunft (1979), in: Daniel Defert/François Lagrange (Hg.): Analytik der Macht, Frankfurt a.M.: Suhrkamp, S. 188-219.

Foucault, Michel (2005[1980-1988]): Über sich selbst schreiben, in: Daniel Defert/François Ewald (Hg.): Schriften in vier Bänden. Dits et Ecrits, Band IV: 1980-1988. Frankfurt a.M.: Suhrkamp, S. 503-521.

Foucault, Michel (2005a[1980-1988]): Subjekt und Macht, in: Daniel Defert/François Ewald (Hg.): Schriften in vier Bänden. Dits et Ecrits, Band IV:1980-1988. Frankfurt a.M.: Suhrkamp, S. 269-294.

Foucault, Michel (2005b[1980-1988]): Gespräch mit Ducio Trombadori, in: Daniel Defert/François Ewald (Hg.): Schriften in vier Bänden. Dits et Ecrits, Band IV: 1980-1988. Frankfurt a.M.: Suhrkamp, S. 51-119.

Foucault, Michel (2005c[1980-1988]): Wahrheit, Macht, Selbst, in: Daniel Defert/François Ewald (Hg.): Schriften in vier Bänden. Dits et Ecrits, Band IV: 1980-1988. Frankfurt a.M.: Suhrkamp, S. 959-966.

Foucault, Michel (2005d[1980-1988]): Subjektivität und Wahrheit, in: Daniel Defert/François Ewald (Hg.): Schriften in vier Bänden. Dits et Ecrits, Band IV: 1980-1988. Frankfurt a.M.: Suhrkamp, S. 258-265.

Foucault, Michel (2005e[1980-1988]): Die Maschen der Macht, in: Daniel Defert/François Ewald (Hg.): Schriften in vier Bänden. Dits et Ecrits, Band IV: 1980-1988. Frankfurt a.M.: Suhrkamp, S. 224-245.

Foucault, Michel; Defert, Daniel; Ewald, François; Lemke, Thomas (2005): Analytik der Macht. Frankfurt a.M.: Suhrkamp.

Foucault, Michel (2010): Neoliberale Gouvernementalität II - Die Theorie des $\mathrm{Hu}$ mankapitals, in: Ulrich Bröckling (Hg.): Kritik des Regierens. Schriften zur Politik. Frankfurt a.M.: Suhrkamp. S. 177-203.

Franklin, Benjamin (1916[1793]): Autobiography of Benjamin Franklin. New York: Henry Holt.

Frederick, Christine (1922): »The New Housekeeping. Efficiency Studies in Home Management«. Berlin: Springer.

Freudenberger, Herbert J. (1974): Staff burn-out, in: Journal of Social Issues, 30 (1), S. 159-165.

Friebe, Holm; Lobo, Sascha (2006): Wir nennen es Arbeit - Die digitale Bohème oder intelligentes Leben jenseits der Festanstellung. München: Heyne.

Gray, Glen L.; Alles, Michael (2015): Data Fracking Strategy, in: Management Accounting Quarterly 16. (3), S. 22-33. Online unter: https://www.thefreelibrar y.com/Data+fracking+strategy\%3A+why+management+accountants+need+ita0429090804 (zuletzt aufgerufen am 17.08.2018). 
Gehring, Petra (2009): Adolphe Quetelet: Sprache und Wirklichkeitsmacht der Bevölkerungsstatistik, in: Alfred Nordmann/Astrid Schwarz (Hg.): Das bunte Gewand der Theorie. Vierzehn Begegnungen mit philosophierenden Forschern. Freiburg i.Br.: Verlag Karl Alber, S. 96-113.

Giedion Sigfried (1982): Die Herrschaft der Mechanisierung. Ein Beitrag zur anonymen Geschichte, Frankfurt a.M.: Europäische Verlagsanstalt.

Gilberth, Frank Bunker (1925): Das ABC der wissenschaftlichen Betriebsfürhung. Bearbeitet von Colin Ross, Berlin: Springer.

Gitelman, Lisa; Jackson, Virginia (2013): »Raw Data« is an Oxymoron. Cambridge und Massachusetts: MIT Press.

Glaser, Barney; Strauss, Anselm L. (2005[1967]): Grounded Theory. Strategien qualitativer Sozialforschung. Bern: Huber.

Goggin und Newell (2005): Foucault on the Phone: Disability and the Mobility of Gouvernment, in: Shelley Tremain (Hg.): Foucault and the Gouvernment of Disability. Michigan: University of Michigan Press, S. 261-280.

Goldscheid, Rudolf (1911): Höherentwicklung und Menschenökonomie. Grundlegung der Sozialbiologie, Leipzig: Klinkhardt.

Gould, Stephen J. (1983): Der falsch vermessene Mensch. Basel, Boston [u.a.]: Birkhäuser Verlag.

Greco, Monica (1993): Psychosomatic Subjects and the »Duty to Be Well«: Personal Agency Within Medical Rationality, in: Economy \& Society, Vol. 22 (3), S. 357372.

Hachtmann, Rüdiger (2011): Fordismus, in Docupedia. Online unter: http:/ /docupedia.de/zg/hachtmann_fordismus_vi_de_2011 (zuletzt aufgerufen am 17.08.2018).

Hacking, Ian (1982): Biopower And The Avalance Of Printed Numbers, in: Humanities in Society 5, S. 279-295.

Hacking, Ian (1986): Making Up People, in: Thomas C. Heller/Christine Brooke-Rose (Hg.): Reconstructing individualism. Autonomy, individuality, and the self in Western thought. Stanford, California: Stanford University Press, S. 222-236.

Hacking, Ian (1990): The taming of chance. Cambridge, New York: Cambridge University Press.

Hacking, Ian (2009): The emergence of probability. Philosophical study of early ideas about probability, induction and statistical inference. Cambridge: Cambridge University Press.

Hardt, Michael; Negri, Antonio (2002): Empire. Die neue Weltordnung. Frankfurt a.M.: Campus-Verlag.

Hartmann, Detlef (2016): McKinsey - das Selbst - der Klassenkampf, in: Felix Klopotek/Peter Scheiffele (Hg.): On time Run - Zonen der Selbstoptimierung. Berichte aus der Leistungsgesellschaft. Berlin: Matthes und Seitz, S. 135-150. 
Heintz, Bettina (2007): Zahlen, Wissen, Objektivität: Wissenschaftssoziologische Perspektiven, in: Andreas Mennicken/Hendrik Vollmer (Hg.): Zahlenwerk. Kalkulation, Organisation und Gesellschaft. Wiesbaden: VS-Verlag, S. 65-87.

Heintz, Bettina (2010): Welterzeugung durch Zahlen. Modell politischer Differenzierung in internationalen Statistiken 1928-2010, in: Soziale Systeme $18(1+2)$, S. 7-39.

Heintz, Bettina (2016): „Wir leben im Zeitalter der Vergleichung«. Perspektiven einer Soziologie des Vergleichs, in: Zeitschrift für Soziologie 45 (5), S. 305-323.

Henckel, Dietrich (2008): „Creative Class in Berlin - Studie über Branchenstrukturen und Standortverhalten der Berliner Kreativwirtschaft « - Studie im Auftrag des Instituts für Stadt- und Regionalplanung der TU Berlin sowie ORCO Germany und Berlin Partner. Online unter: https://docplayer.org/8524436-Cre ative-class-in-berlin-studie-ueber-branchenstrukturen-und-standortverhalte n-der-berliner-kreativwirtschaft.html (zuletzt aufgerufen am 17.08.2018).

Herkommer, Benjamin; Henckel, Dietrich; Zahn, Anja (2010): Kreativwirtschaft in Berlin: Standorte und Standortverhalten der Unternehmen, in: Matthias Schmidt/Christoph Schank (Hg.): Metropolregion Berlin vor demografischen und gesellschaftlichen Herausforderungen. München und Mehring: Rainer Hamp Verlag. S. 121.

Herrmann, Hans-Christian von (2002): Pensum - Spur - Code. Register der Arbeitswissenschaft bei Taylor, Gilbreth und Bernstein, in: Ulrich Bröckling/Eva Horn (Hg.): Anthropologie der Arbeit. Tübingen: Narr, S. 193-208.

Hilpert, Thilo (1995): Die postfordistische Stadt. Suche nach einer Gestaltkultur der offenen Form, in: Regina Bittner (Hg.): Zukunft aus Amerika, Fordismus in der Zwischenkriegszeit, Siedlung, Stadt, Raum. Dessau: Stiftung Bauhaus Dessau, S. 135-147.

Hilpert, Thilo (1978): Die funktionelle Stadt. Le Corbusiers Stadtvision - Bedingungen, Motive, Hintergründe, Braunschweig: Vieweg Verlag.

Hine, Christine M. (2000): Virtual Ethnography. London: Sage.

Hirschauer, Stefan; Amann, Klaus (1997): Die Befremdung der eigenen Kultur: Zur ethnographischen Herausforderung soziologischer Empirie. Frankfurt a.M.: Suhrkamp.

Hutter, Michael; Teubner, Gunther (1994): Der Gesellschaft fette Beute. Homo juridicus und homo oeconomicus als kommunikationserhaltende Fiktionen, in: Peter Fuchs/Andreas Göbel (Hg.): Der Mensch - das Medium der Gesellschaft, Frankfurt a.M.: Suhrkamp, S. 110-145.

Jäger, Siegfried (1999): Kritische Diskursanalyse. Eine Einführung. Münster: Unrast-Verlag.

Johnson, Andrew (2014): Foucault: Critical Theory of the Police in a Neoliberal Age, in: Theoria, Volume 61 (141), S. 5-29. 
Marx, Karl (1974[1867]): Das Kapital. Kritik der politischen Ökonomie, Band I - Der Produktionsprozess des Kapitals. Berlin: Dietz Verlag.

Kailer, Thomas (2011): Vermessung des Verbrechers. Die Kriminalbiologische Untersuchung in Bayern 1923-1945. Bielefeld: transcript.

Keller, Reiner (2004): die vielgestaltige praxis der diskursforschung - eine einführung, in: ders. (Hg.): Handbuch Sozialwissenschaftliche Diskursanalyse. Band 2 - Forschungspraxis. Wiesbaden: VS-Verlag. S. 7-18.

Kerchner, Brigitte; Schneider, Silke (2006): »Endlich Ordnung in der Werkzeugkiste«. Zum Potenzial der Foucaultschen Diskursanalyse für die Politikwissenschaft - Einleitung, in: Brigitte Kerchner (Hg.): Foucault: Diskursanalyse der Politik. Eine Einführung. Wiesbaden: VS-Verlag.

Klinger, Cornelia (1995): Flucht, Trost, Revolte. Die Moderne und ihre ästhetischen Gegenwelten. München, Wien: C. Hanser Verl.

Klopotek, Felix; Scheiffele, Peter (2016): On time Run - Zonen der Selbstoptimierung. Berichte aus der Leistungsgesellschaft. Berlin: Matthes und Seitz.

Knorr-Cetina, Karin (2012): Die Fabrikation von Erkenntnis. Zur Anthropologie der Naturwissenschaft. Frankfurt a.M.: Suhrkamp.

Köhler, Benedikt (2008): Amtliche Statistik, Sichtbarkeit und die Herstellung von Verfügbarkeit, in: Berliner Journal für Soziologie, 18 (1), S. 73-98.

Lemke, Thomas (2000): Neoliberalismus, Staat und Selbsttechnologien. Ein kritischer Überblick über die governmentality studies, in: Politische Vierteljahresschrift: Zeitschrift der Deutschen Vereinigung für Politische Wissenschaft, 41 (1), S. 31-47.

Lemke, Thomas (2007): Gouvernementalität und Biopolitik, Wiesbaden: VS-Verlag. Lemke, Thomas (2010): Die Regierung der Risiken. Von der Eugenik zur genetischen Gouvernementalität, in: Ulrich Bröckling/Susanne Krasmann/Thomas Lemke (Hg.): Gouvernementalität der Gegenwart. Studien zur Ökonomisierung des Sozialen. Frankfurt a.M.: Suhrkamp.

Liepitz, Alain (1986): Nach dem Ende des »Goldenen Zeitalters«. Regulation und Transformation kapitalistischer Gesellschaften, in Hans-Peter Krebs (Hg.): Argument Sonderband 255, Berlin: Argument-Verlag.

Link, Jürgen (1997): Versuch über den Normalismus. Wie Normalität produziert wird. Opladen: Westdeutscher Verlag.

Link, Jürgen (1999): Wie das Kügelchen fällt und das Auto rollt. Zum Anteil des Normalismus an der Identitätsproblematik in der Moderne. In: Herbert Willems und Alois Hahn (Hg.): Identität und Moderne. Frankfurt a.M.: Suhrkamp, S. 164-179.

Link, Jürgen (2001): Aspekte der Normalisierung von Subjekten. Kollektivsymbolik, Kurvenlandschaften, Infografiken, in: Ute Gerhard, Jürgen Link und Ernst Schulte-Holtey (Hg.): Infografiken, Medien, Normalisierung. Zur Kartografie politisch-sozialer Landschaften. Heidelberg: Synchron, S. 77-92. 
Link, Jürgen (2012): Wie man auf »780/800 fuckability« kommt. Zum Verhältnis von Crowdsourcing, Datenrevolution und Normalismus, in: Birgit Riegraf/Dierk Spreen/Sabine Mehlmann (Hg.): Medien, Körper, Geschlecht. Diskursivierungen von Materialität. Bielefeld: transcript. S. 37-50.

Lupton, Deborah (2013): Quantifying the body: monitoring and measuring health in the age of mHealth technologies, in: Critical Public Health 23 (4), S. 393-403.

Lupton, Deborah (2014): Critical Perspectives on Digital Health Technologies, in: Sociology Compass 8 (12), S. 1344-1359.

Lupton, Deborah (2016): The quantified self. Cambridge: Polity Press.

Lupton, Deborah (2016b): You are your data: Self-tracking practices and concepts of data, in: Selke, Stefan (Hg.): Lifelogging: Theoretical approaches and case studies about self-tracking. New York: VS-Verlag, S. 61-79.

Lüders, Jenny (2007): Ambivalente Selbstpraktiken. Eine Foucault'sche Perspektive auf Bildungsprozesse in Weblogs. Bielefeld: transcript.

Manske, Alexandra (2006): Die Stellung halten. Marktstrategien und Positionskämpf in Berlins Internetbranche, in: Soziale Welt 57 (2), S. 157-175.

Manske, Alexandra (2007): Prekarisierung auf hohem Niveau. Eine Feldstudie über Alleinunternehmer in der IT-Branche. München und Mehring: Rainer Hampp Verlag.

Manske, Alexandra (2013): Kreative als aktivierte Wirtschaftsbürger. Zur wohlfahrtsstaatlichen Rahmung von künstlerisch-kreativer Arbeit, in: Östereichische Zeitschrift für Soziologie 38 (3), S. 259-276.

Mattissek, Annika (2010): Stadtmarketing in der neoliberalen Stadt. Potentiale von Gouvernementalitäts- und Diskursanalyse für die Untersuchung aktueller Prozesse der Stadtentwicklung, in: Johannes Angermüller (Hg.): Diskursanalyse meets Gouvernementalitätsforschung. Perspektiven auf das Verhältnis von Subjekt, Sprache, Macht und Wissen. Frankfurt a.M. [u.a.]: Campus-Verlag, S. 129-155.

Mämecke, Thorben; Wehner, Josef (2014): „Staatistik« - Zur Vermessung politischer Beteiligung, in: Kathrin Voss (Hg.): Internet und Partizipation. Bottom-up oder Top-down? Politische Beteiligungsmöglichkeiten im Internet. Wiesbaden: VSVerlag, S. 311-322.

Mämecke, Thorben (2016): Benchmarking the self - Kompetetive Selbstvermessung im betrieblichen Gesundheitsmanagement, in: Stefanie Duttweiler/Robert Gugutzer/Jan-Hendrik Passoth/Jörg Strübing (Hg.): Leben nach Zahlen. Self-Tracking als Optimierungsprojekt? Bielefeld: transcript, S. 103-122.

McGee, Micki (2005): Self-Help, Inc. Makeover Culture in American Life. Oxford: Oxford University Press.

Meier, Stefan (2008): (Bild-)Diskurs im Netz. Konzept und Methode für eine semiotische Diskursanalyse im Word Wide Web. Köln: Halem. 
Meißner, Stefan (2016): Selbstoptimierung durch Quantified Self? Selbstvermessung als Möglichkeit von Selbststeigerung, Selbsteffektivierung und Selbstbegrenzung, in: Stefan Selke (Hg.): Lifelogging - Digitale Selbstvermessung und Lebensprotokollierung zwischen disruptiver Technologie und kulturellem Wandel. Wiesbaden: VS-Verlag, S. 217-236.

Moldaschl, Manfred; Sauer, Dieter (2000): Internalisierung des Marktes. Zur neuen Dialektik von Kooperation und Herrschaft, in: Heiner Minssen (Hg.): Begrenzte Entgrenzung- Wandlungen von Organisation und Arbeit. Berlin: Edition Sigma, S. 205-224.

Moore, Phoebe; Piwek, Lukasz (2017): Regulating wellbeing in the brave new quantified workplace, in: Employee Relations 39 (3), S. 308-316.

Moore, Phoebe (2018): The Quantified Self in Precarity: Work, Technology and What Counts. London: Taylor and Francis.

Moore, Phoebe; Roper, Ian; Piwek, Lukasz (2018): The Quantified Workplace: A Study in Self-Tracking, Agility and Change Management, in: Btihaj Ajana (Hg.): Self-tracking. Empirical and philosophical investigations. London [u.a.]: Palgrave Macmillan, Cham, S. 93-110.

Morris, Paul (1991): Freeing the spirit of enterprise. The gegesis and development of the concept of enterprise culture, in: Russel Keat, Nicholas Abercrombie (Hg.): Enterprise Culture, London und New York. S. 21-37.

Muhle, Maria (2008): Eine Genealogie der Biopolitik. Zum Begriff des Lebens bei Foucault und Canguilhem. Bielefeld: transcript.

Mürner, Christian; Sierck, Udo (2009): Krüppelzeitung. Brisanz der Behindertenbewegung. Neu-Ulm: AG-SPAK-Bücher.

Nachtwey, Oliver; Staab Philipp (2016): Die Avantgarde des digitalen Kapitalismus, in: Mittelweg 36 (6), S. 1-33.

Neckel, Sieghardt; Dröge, Kai; Somm, Irene (2004): Welche Leistung, welche Leistungsgerechtigkeit?, in: Berger Peter A./Schmidt Volker. H. (Hg.): Welche Gleichheit, welche Ungleichheit? Reihe »Sozialstrukturanalyse« Band 20. Wiesbaden: VS-Verlag.

Neckel, Sighard; Wagner Greta (2013): Einleitung: Leistung und Erschöpfung, in: dies. (Hg.): Leistung und Erschöpfung. Burnout in der Wettbewerbsgesellschaft. Berlin: Suhrkamp.

Neckel, Sighard (2014): Emotionale Reflexivität - Paradoxien der Emotionalisierung, in: Thilo Fehmel, Stephan Lessenich und Jenny Preunkert (Hg.): Systemzwang und Akteurswissen. Theorie und Empirie von Autonomiegewinnen. Frankfurt a.M. und New York: Campus-Verlag, S. 117-129.

Neocleous, Mark (2000): The Fabrication of Social Order: A Critical Theory of Police Power. London: Pluto Press.

Pieper, Marianne (2006): Diskursanalysen - Kritische Analytik der Gegenwart und wissenspolitische Deutungsmusteranalyse. Ein Kommentar zu den Beiträgen 
von Susanne Krasmann und Julia Lepperhoff, in: Brigitte Kerchner (Hg.): Foucault: Diskursanalyse der Politik. Eine Einführung. Wiesbaden: VS-Verlag.

Pongratz, Hans Jürgen; Voß, Günter (2003): Arbeitskraftunternehmer. Erwerbsorientierungen in entgrenzten Arbeitsformen. Berlin: Edition Sigma.

Porter, Theodore M. (1995): Trust in numbers. The pursuit of objectivity in science and public life. Princeton, N.J: Princeton University Press.

Pritz, Sarah Miriam (2016): Mood Tracking: Zur digitalen Selbstvermessung der Gefühle, in: Stefan Selke (Hg.): Lifelogging - Digitale Selbstvermessung und Lebensprotokollierung zwischen disruptiver Technologie und kulturellem Wandel. Wiesbaden: VS-Verlag, S. 127-150.

Püschel, Florian (2014): Big Data und die Rückkehr des Positivismus. Zum gesellschaftlichen Umgang mit Daten, in: Marcus Burkhardt, Sebastian Gießmann (Hg.): Mediale Kontrolle 2014 (3.1). Online unter: www.medialekontrolle.de/au sgaben/3-12014-datenkritik/ (zuletzt aufgerufen am 17.08.2018).

Rappaport, Julian (1981): In Praise of Paradox: A Social Policy of Empowerment over Prevention, in: American Journal of Community Psychology, 9 (1), S. 1-25.

Reckwitz, Andreas (2010): Das hybride Subjekt. Eine Theorie der Subjektkulturen von der bürgerlichen Moderne zur Postmoderne. Weilerswist: Velbrück Wissenschaft.

Reckwitz, Andreas (2010b): Unscharfe Grenzen. Bielefeld: transcript.

Reckwitz, Andreas (2011): Die Erfindung der Kreativität. Zum Prozess gesellschaftlicher Ästhetisierung. Berlin: Suhrkamp.

Reichert, Ramón (2017): Big Data als Boundary Objects. Zur medialen Epistemologie von Daten, in: Thorben Mämecke/Jan-Hendrik Passoth/Josef Wehner (Hg.): Bedeutende Daten. Modelle, Verfahren und Praxis der Vermessung und Verdatung im Netz. Wiesbaden: VS-Verlag, S. 17-34.

Unternährer, Markus (2016): Selbstquantifizierung als numerische Form der Selbstthematisierung, in: Stefanie Duttweiler/Robert Gugutzer, Jan-Hendrik Passoth/Jörg Strübing (Hg.): Leben nach Zahlen. Self-Tracking als Optimierungsprojekt? Bielefeld: transcript, S. 201-221.

Rheingold, Howard (1993): The virtual community. Homesteading on the electronic frontier. Reading, Massachusetts: Addison-Wesley. Online unter: http://rheing old.com/vc/book/intro.html (zuletzt aufgerufen am 17.08.2018).

Rosa, Hartmut (2009): Von der stabilen Position zur dynamischen Performanz. Beschleunigung und Anerkennung in der Spätmoderne, in: Rainer Forst, Martin Hartmann/Rahel Jaeggi/Martin Saar (Hg.): Sozialphilosophie und Kritik. Frankfurt a.M.: Suhrkamp, S. 655-671.

Rose, Nikolas (1996): Inventing Our Selves. Psychology, Power and Personhood. New York: Cambridge University Press.

Rose, Nikolas (1999): Powers of Freedom. Reframing Political Thought. Cambridge: Cambridge University Press. 
Rose, Nikolas (2000): Das Regieren von unternehmerischen Individuen (deutsche Übersetzung des Kapitels »Governing Enterprising Individuals« aus »Inventing Our Selves«), in: Kurswechsel Zeitschrift für gesellschafts-, wirtschafts- und umweltpolitische Alternativen 2, S. 8-27.

Rose, Nikolas; Novas Carlos (2000): Genetic risk and the birth of the somatic individual, in: Economy and Society 29 (4), S. 485-513.

Röcke, Anja (2017): (Selbst)Optimierung. Eine soziologische Bestandsaufnahme, in: Berliner Journal für Soziologie. Wiesbaden: VS-Verlag, S. 1-17.

Röhle, Theo (2010): Der Google-Komplex. Über Macht im Zeitalter des Internets. Bielefeld: transcript.

Röhle, Theo (2017): »Data should be cooked with care« - Digitale Kartographie zwischen Akkumulation und Aggregation, in: Thorben Mämecke/Jan-Hendrik Passoth/Josef Wehner (Hg.): Bedeutende Daten. Modelle, Verfahren und Praxis der Vermessung und Verdatung im Netz. Wiesbaden: VS-Verlag, S. 71-90.

Saar, Martin (2003) Nachwort, in: ders. (Hg.): Foucault, Michel. Die Wahrheit und die juristischen Formen. [1973]. Frankfurt a.M.: Suhrkamp.

Saar, Martin (2007): Macht, Staat, Subjektivität. Foucaults >Geschichte der Gouvernementalität im Werkkontext, in: Krasmann, Susanne/Michael Volkmer (Hg.): Michel Foucaults »Geschichte der Gouvernementalität« in den Sozialwissenschaften. Internationale Beiträge, Bielefeld: transcript, S. 23-45.

Saldern, Adelheid von (1991): "Statt Kathedralen die Wohnmaschine«. Paradoxien der Rationalisierung im Kontext der Moderne, in: Frank Bajohr, Werner Johe, Uwe Lohalm (Hg.): Zivilisation und Barbarei. Hamburg: Ergebnisse Verlag, S. 168-192.

Saldern, Adelheid von; Hachtmann, Rüdiger (2009): Das fordistische Jahrhundert. Eine Einleitung, in: Zeithistorische Forschungen/Studies in Contemporary History, Online-Ausgabe, 6 (2). S. 174-185.

Sarasin, Philipp (2001): Reizbare Maschinen. Eine Geschichte des Körpers 17651914. Frankfurt a.M.: Suhrkamp.

Schaupp, Simon (2016): Die Vermessung des Unternehmers seiner selbst. Vergeschlechtliche Quantifizierung im Diskurs des Self-Tracking, in: Stefan Selke (Hg.): Lifelogging - Digitale Selbstvermessung und Lebensprotokollierung zwischen disruptiver Technologie und kulturellem Wandel. Wiesbaden: VS-Verlag. S. 151-170.

Schäfer, Armin (2004): ... und das Wort ist Fleisch geworden. Diskurse der Biopolitik, in: Ulrich Bröckling/Benjamin Bühler/Marcus Hahn/Matthias Schöning/Manfred Weinberg (Hg.): Disziplinen des Lebens. Zwischen Anthropologie, Literatur und Politik. Tübingen: Gunter Narr Verlag.

Schmechel, Corinna (2016): Kalorienzählen oder tracken? Wie Quantified Self feminisierte Körperpraxen zu Männlichkeitsperformanzen transformiert, in: Stefan Selke (Hg.): Lifelogging - Digitale Selbstvermessung und Lebensprotokollie- 
rung zwischen disruptiver Technologie und kulturellem Wandel. Wiesbaden: VS-Verlag, S. 171-192.

Schmidbauer, Wolfgang (2012): Mehr Hofnarr als Hofrat. Über die Krisen der Psychotherapie, in Kursbuch 170 »Krisen Lieben«, Hamburg: Murmann. S. 150-173. Schmidt-Burkhardt, Astrit (2017): Die Kunst der Diagrammatik. Perspektiven eines neuen bildwissenschaftlichen Paradigmas. Bielefeld: transcript.

Schultz, Theodor W. (1986[1899]): In Menschen investieren. Die Ökonomie der Bevölkerungsqualität, Tübingen: Mohr-Siebeck.

Schütz, Alfred (1972): Der Fremde, in: Arvid von Brodersen (Hg.): Gesammelte Aufsätze. II Studien zur soziologischen Theorie. Den Haag: Martinus Nijhoff. S. 5369.

Schwap-Trapp, Michael (2006): Diskurs als soziologisches Konzept. Bausteine für eine soziologisch orientierte Diskursanalyse, in: Reiner Keller (Hg.): Handbuch sozialwissenschaftliche Diskursanalyse. Theorien und Methoden. Wiesbaden: VS-Verlag, S. 263-285.

Selke, Stefan (2016): Lifelogging zwischen disruptiver Technologie und kulturellem Wandel, in: Stefan Selke (Hg.): Lifelogging - Digitale Selbstvermessung und Lebensprotokollierung zwischen disruptiver Technologie und kulturellem Wandel. Wiesbaden: VS-Verlag.

Sennett, Richard (2000): Der flexible Mensch. Die Kultur des neuen Kapitalismus. München: Siedler.

Sontag, Susan (1981): Krankheit als Metapher. Frankfurt a.M.: Fischer Taschenbuch Verlag.

Spilker, Niels (2010): Die Regierung der Prekarität. Zur neoliberalen Konzeption unsicherer Arbeitsverhältnisse. Münster: Unrast-Verlag.

Starker, Steven (1989): Oracle at the Supermarket: The American Preoccupation with Self-Help Books. New Brunswick und Oxford: Transaction.

Straub, Jürgen; Sieben, Anna; Sabisch-Fechtelpeter, Katja (2012): Menschen besser machen. Terminologische und theoretische Aspekte vielgestaltiger Optimierungen des Humanen, in: Anna Sieben/Katja Sabisch-Fechtelpeter/Jürgen Straub (Hg.): »Menschen machen«. Die hellen und die dunklen Seiten humanwissenschaftlicher Optimierungsprogramme Bielefeld: transcript. S. 27-78.

Taylor, Frederick Winslow (1977[1922]): Die Grundsätze wissenschaftliche Betriebsführung. Rudolf Roesler, (Hg.). München und Berlin: Psychologie-VerlagsUnion.

Thibaud, Paul (1985): The Triumph of the Entrepreneur, in: Telos, Nr. 64. Jahrgang 1985. S. 134-140.

Vormbusch, Uwe (2012): Die Herrschaft der Zahlen. Zur Kalkulation des Sozialen in der kapitalistischen Moderne, Frankfurt a.M. und New York: Campus Verlag. Vormbusch, Uwe (2018): Taxonomien des Selbst. Zur Hervorbringung subjektbezogener Bewertungsordnungen im Kontext ökonomischer und kultureller Unsi- 
cherheit, in: Stefanie Duttweiler/Robert. Gugutzer/Jan-Hendrik Passoth/Jörg. Strübing (Hg.): Leben nach Zahlen. Self-Tracking als Optimierungsprojekt. Bielefeld: transcript, S. 45-62.

Vormbusch, Uwe; Kappler, Karolin (2018): Leibschreiben. Zur medialen Repräsentation des Körperleibes im Feld der Selbstvermessung, in: Thorben Mämecke/Jan-Hendrik Passoth/Josef Wehner (Hg.): Bedeutende Daten. Modelle, Verfahren und Praxis der Vermessung und Verdatung im Netz. Wiesbaden: VSVerlag, S. 207-231.

Voß, G. Günter; Pongratz, Hans J. (1998): Der Arbeitskraftunternehmer. Eine neue Grundform der Ware Arbeitskraft?, in: Kölner Zeitschrift für Soziologie und Sozialpsychologie 50 (1). S. 131-158.

Waldschmidt, Anne (2004): Der Humangenetik-Diskurs der Experten: Erfahrungen mit dem Werkzeugkasten der Diskursanalyse, in: Reiner Keller (Hg.): Handbuch Sozialwissenschaftliche Diskursanalyse. Band 2 - Forschungspraxis. Wiesbaden: VS Verlag, S. 149-170.

Waring, Marilyn (1999[1988]): Counting for nothing. What men value and what women are worth. Toronto, Buffalo: University of Toronto Press.

Weber, Jutta (2017): Pleasing Little Sister. Big Data und Social Media Surveillance, in: Thorben Mämecke/Jan-Hendrik Passoth/Josef Wehner (Hg.): Bedeutende Daten - Modelle, Verfahren und Praxis der Vermessung und Verdatung im Netz. Wiesbaden: VS-Verlag, S. 91-104.

Weber, Max (1973[1934]): Die protestantische Ethik und der Geist des Kapitalismus. Tübingen: Mohr-Siebeck.

Weber, Max (1980[1922]): Wirtschaft und Gesellschaft. Grundriss der verstehenden Soziologie, Tübingen: Mohr-Siebeck.

Wehner, Josef; Passoth, Jan-Hendrik (2013): Quoten, Kurven und Profile. Zur Vermessung des sozialen Welt. Wiesbaden: VS-Verlag.

Weisgerber, Corrine und Butler, Shannan H. (2016): Curating the Soul: Foucault's concept of hupomnemata and the digital technology of self-care, Information, Communication \& Society, 19 (10), S. 1340-1355.

Wiedemann, Lisa (2016a): Datensätze der Selbstbeobachtung - Daten verkörpern und Leib vergessen!?, in: Stefan Selke (Hg.): Lifelogging. Digitale Selbstvermessung und Lebensprotokollierung zwischen disruptiver Technologie und kulturellem Wandel. Wiesbaden: VS-Verlag, S. 65-93.

Wiedemann, Lisa (2016b): „Vom Piksen zum Scannen, vom Wert zu Daten« - Digitalisierte Selbstvermessung im Kontext Diabetes, in: Stefanie Duttweiler/Robert Gugutzer/Jan-Hendrik Passoth/Jörg Strübing (Hg.): Leben nach Zahlen. Self-Tracking als Optimierungsprojekt?, Bielefeld: transcript, S. 293-324.

Witte, Irene (1922): Die rationelle Haushaltsführung. Betriebswissenschaftliche Studien. Berlin: VS-Verlag.

Witte, Irene (1928): Heim und Technik in Amerika. Berlin: VS-Verlag. 
Wupper-Tewes, Hans (2002): Die Normalisierung industrieller Arbeit. Leistung, Norm und Gesundheit in der Rationalisierungsbewegung der Weimarer Republik, in: Ulrich Bröckling und Eva Horn (Hg.): Anthropologie der Arbeit. Tübingen: Narr, S. 97-109.

Zillien, Nicole; Fröhlich, Gerrit; Kofahl, Daniel (2016): Ernährungsbezogene Selbstvermessung. Von der Diätetik bis zum Diet Tracking, in: Stefanie Duttweiler/Robert Gugutzer/Jan-Hendrik Passoth/Jörg Strübing (Hg.): Leben nach Zahlen. Self-Tracking als Optimierungsprojekt? Bielefeld: transcript, S. 123140.

Zillien, Nicole, Fröhlich, Gerrit (2018): Reflexive Selbstverwissenschaftlichung. Eine empirische Analyse der digitalen Selbstvermessung, in: Thorben Mämecke/JanHendrik Passoth/Josef Wehner (Hg.): Bedeutende Daten. Modelle, Verfahren und Praxis der Vermessung und Verdatung im Netz. Wiesbaden: VS-Verlag, S. 233-251. 


\section{Abkürzungsverzeichnis}

BGM Betriebliches Gesundheitsmanagement

BMI Body-Mass-Index

CDC Center for Disease Control and Prevention

CDI Composite Diversity Index

HRV Herzratenvariabilität

ICD International Classification of Diseases and Related Health Problems

OCD Obsessive-Compulsive Disorder

ST Self-Tracking

QS Quantified Self

QSEU Quantified Self Europe Conference

WHO World Health Organisation 


\section{Medienwissenschaft}

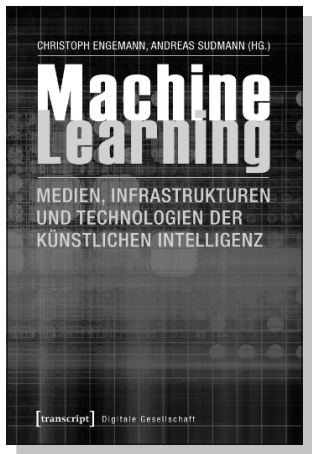

Christoph Engemann, Andreas Sudmann (Hg.)

Machine Learning -

Medien, Infrastrukturen und Technologien

der Künstlichen Intelligenz

2018, 392 S., kart.

$32,99 €(D E), 978-3-8376-3530-0$

E-Book:

PDF: $32,99 €$ (DE), ISBN 978-3-8394-3530-4

EPUB: $32,99 €$ (DE), ISBN 978-3-7328-3530-0

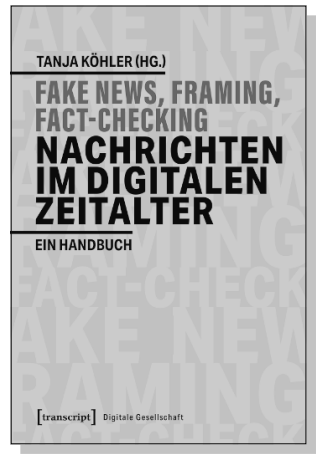

Tanja Köhler (Hg.)

Fake News, Framing, Fact-Checking: Nachrichten im digitalen Zeitalter Ein Handbuch

Juni 2020, 568 S., kart., 41 SW-Abbildungen 39,00€ (DE), 978-3-8376-5025-9

E-Book:

PDF: 38,99 € (DE), ISBN 978-3-8394-5025-3

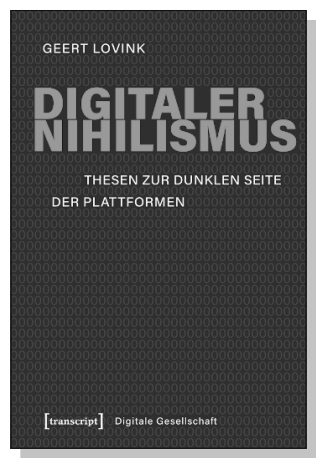

Geert Lovink

\section{Digitaler Nihilismus}

Thesen zur dunklen Seite der Plattformen

2019, 242 S., kart.

24,99 € (DE), 978-3-8376-4975-8

E-Book:

PDF: $21,99 €$ (DE), ISBN 978-3-8394-4975-2

EPUB: $21,99 €$ (DE), ISBN 978-3-7328-4975-8 


\section{Medienwissenschaft}
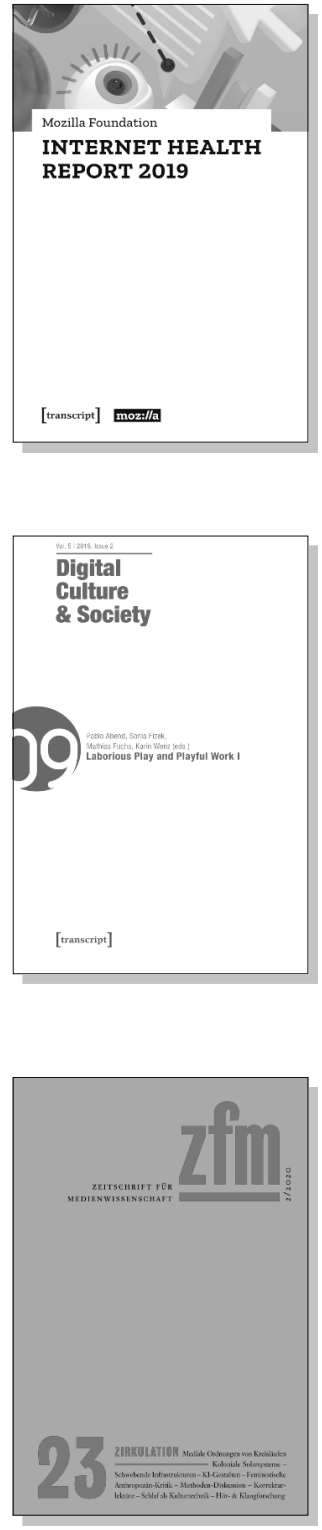

Mozilla Foundation

Internet Health Report 2019

2019, 118 p., pb., ill.

19,99€ (DE), 978-3-8376-4946-8

E-Book: available as free open access publication

PDF: ISBN 978-3-8394-4946-2

Pablo Abend, Sonia Fizek, Mathias Fuchs, Karin Wenz (eds.)

Digital Culture \& Society (DCS)

Vol. 5, Issue 2/2019 - Laborious Play and Playful Work I

September 2020, 172 p., pb., ill.

29,99€ (DE), 978-3-8376-4479-1

E-Book:

PDF: 29,99€ (DE), ISBN 978-3-8394-4479-5
Gesellschaft für Medienwissenschaft (Hg.)

\section{Zeitschrift für Medienwissenschaft 23}

Jg. 12, Heft 2/2020: Zirkulation.

Mediale Ordnungen von Kreisläufen

September 2020, 218 S., kart.

24,99€ (DE), 978-3-8376-4924-6

E-Book: kostenlos erhältlich als Open-Access-Publikation PDF: ISBN 978-3-8394-4924-0

ISBN 978-3-7328-4924-6 
